SUPPORTING INFORMATION for

\title{
Hiscotropic Rearrangements: Hybrids of Electrocyclic and Sigmatropic Reactions
}

\author{
Dustin H. Nouri and Dean J. Tantillo* \\ Department of Chemistry \\ University of California, Davis \\ One Shields Avenue \\ Davis, CA 95616 \\ tantillo@chem.ucdavis.edu
}

contents:

p S2: full GAUSSIAN citation

p S3: overview of activation barriers and reaction energies for hiscotropic rearrangements of structures from Scheme 2 (Figure S1).

pp S4-S170: coordinates, energies and IRC plots for all computed structures 
full GAUSSIAN citation:

GAUSSIAN03, revision B.04: M. J. Frisch, G. W. Trucks, H. B. Schlegel, G. E. Scuseria, M. A. Robb, J. R. Cheeseman, J. A. Montgomery, Jr., T. Vreven, K. N. Kudin, J. C. Burant, J. M. Millam, S. S. Iyengar, J. Tomasi, V. Barone, B. Mennucci, M. Cossi, G. Scalmani, N. Rega, G. A. Petersson, H. Nakatsuji, M. Hada, M. Ehara, K. Toyota, R. Fukuda, J. Hasegawa, M. Ishida, T. Nakajima, Y. Honda, O. Kitao, H. Nakai, M. Klene, X. Li, J. E. Knox, H. P. Hratchian, J. B. Cross, C. Adamo, J. Jaramillo, R. Gomperts, R. E. Stratmann, O. Yazyev, A. J. Austin, R. Cammi, C. Pomelli, J. W. Ochterski, P. Y. Ayala, K. Morokuma, G. A. Voth, P. Salvador, J. J. Dannenberg, V. G. Zakrzewski, S. Dapprich, A. D. Daniels, M. C. Strain, O. Farkas, D. K. Malick, A. D. Rabuck, K. Raghavachari, J. B. Foresman, J. V. Ortiz, Q. Cui, A. G. Baboul, S. Clifford, J. Cioslowski, B. B. Stefanov, G. Liu, A. Liashenko, P. Piskorz, I. Komaromi, R. L. Martin, D. J. Fox, T. Keith, M. A. Al-Laham, C. Y. Peng, A. Nanayakkara, M. Challacombe, P. M. W. Gill, B. Johnson, W. Chen, M. W. Wong, C. Gonzalez, and J. A. Pople, Gaussian, Inc., Pittsburgh PA, 2003. 

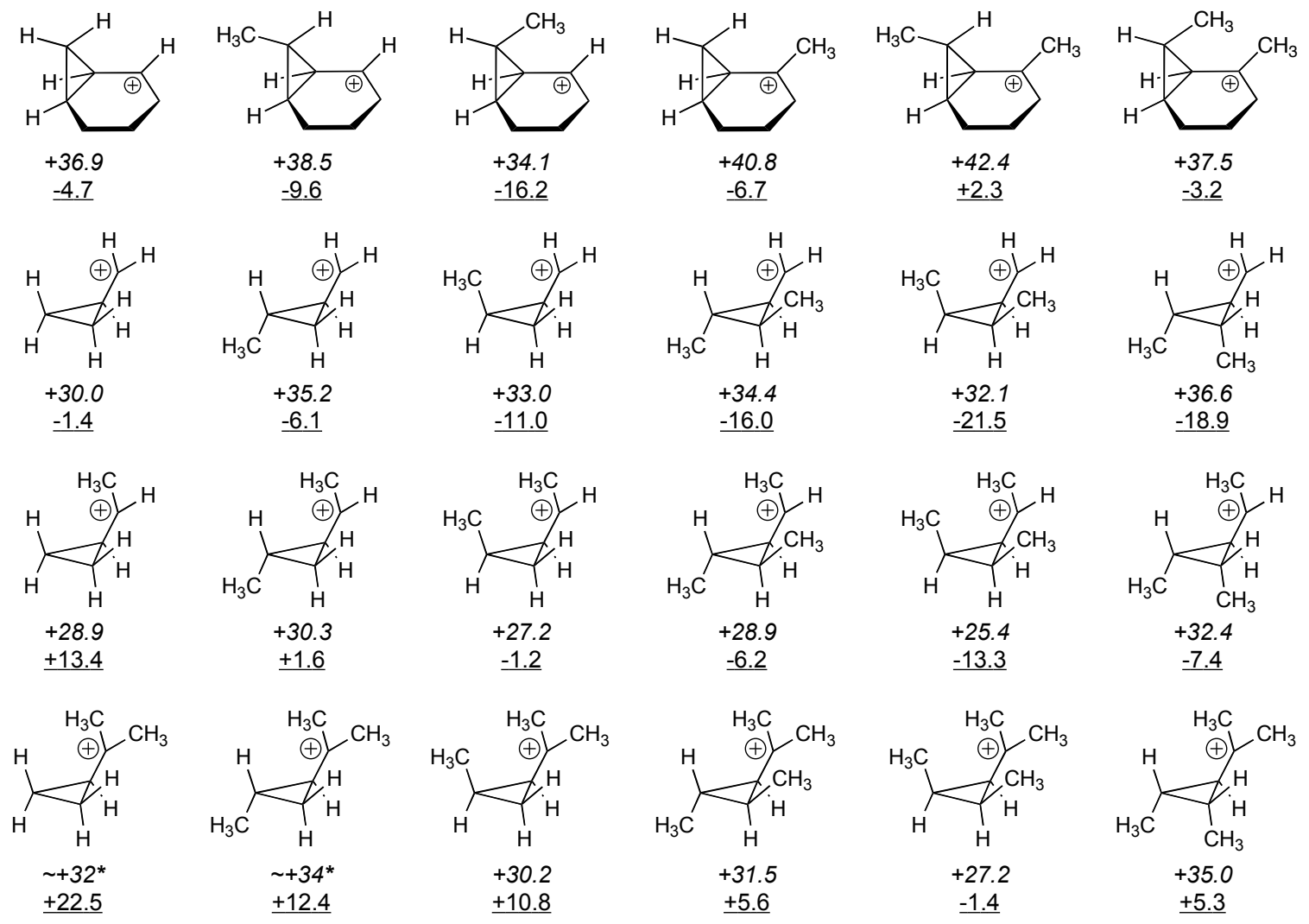

Figure S1. Activation barriers (italics) and reaction energies (underlined) in $\mathrm{kcal} / \mathrm{mol}$ for hiscotropic rearrangements of cyclopropylcarbinyl cations in Scheme 2. Barriers marked with *'s are for reactions that appear to involve intermediates (in very shallow wells); see text for details. $^{42}$ 
b3lyp/6-31G(d)

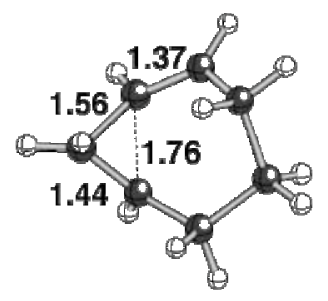

\section{Coordinates:}

Standard orientation:

\begin{tabular}{|c|c|c|c|c|}
\hline \multirow{2}{*}{$\begin{array}{l}\text { Center } \\
\text { Number }\end{array}$} & \multirow{2}{*}{$\begin{array}{l}\text { Atomic } \\
\text { Number }\end{array}$} & \multirow{2}{*}{$\begin{array}{r}\text { Atomic } \\
\text { Type }\end{array}$} & \multicolumn{2}{|c|}{ Coordinates (Angstroms) } \\
\hline & & & X $\quad Y$ & $\quad \mathrm{Z}$ \\
\hline 1 & 6 & 1.689506 & 0.182844 & 0.649914 \\
\hline 2 & 6 & 1.123189 & -0.857626 & -0.361725 \\
\hline 3 & 6 & 0.913595 & 0.889166 & -0.341087 \\
\hline 4 & 1 & 1.425927 & 1.229600 & -1.238394 \\
\hline 5 & 6 & -0.120749 & -1.434745 & -0.275937 \\
\hline 6 & 6 & -0.459427 & 1.455518 & -0.045611 \\
\hline 7 & 1 & -0.625376 & 2.304309 & -0.717145 \\
\hline 8 & 6 & -1.253930 & -0.885571 & 0.503434 \\
\hline 9 & 6 & -1.633227 & 0.466316 & -0.184949 \\
\hline 10 & 1 & -1.866620 & 0.294796 & -1.241586 \\
\hline 11 & 1 & -2.102398 & -1.572868 & 0.496227 \\
\hline 12 & 1 & -0.317789 & -2.279280 & -0.936709 \\
\hline 13 & 1 & -0.446342 & 1.840961 & 0.981287 \\
\hline 14 & 1 & -0.975790 & -0.684992 & 1.546166 \\
\hline 15 & 1 & -2.533788 & 0.871449 & 0.285360 \\
\hline 16 & 1 & 1.282139 & 0.107027 & 1.655869 \\
\hline 17 & 1 & 2.775130 & 0.217967 & 0.601607 \\
\hline 18 & 1 & 1.831169 & -1.224378 & -1.096905 \\
\hline
\end{tabular}

Energy: $H F=-273.0622889$

Zero-Point Energy Correction: 0.164299

Imaginary Frequency: Minimum (No imaginary frequencies) 

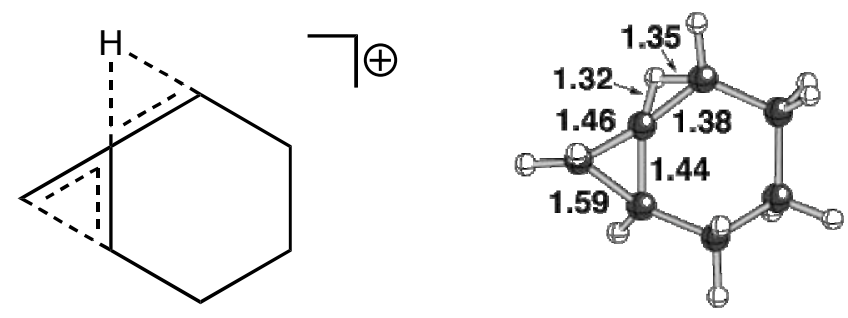

b3lyp/6-31G(d)

\section{Coordinates:}

Standard orientation:

\begin{tabular}{|c|c|c|c|c|c|}
\hline \multirow{2}{*}{$\begin{array}{l}\text { Center } \\
\text { Number }\end{array}$} & \multirow{2}{*}{$\begin{array}{l}\text { Atomic } \\
\text { Number }\end{array}$} & \multirow{2}{*}{\multicolumn{2}{|c|}{$\begin{array}{r}\text { Atomic } \\
\text { Type }\end{array}$}} & \multicolumn{2}{|c|}{ Coordinates (Angstro } \\
\hline & & & & X $\quad Y$ & $\mathrm{Z}$ \\
\hline 1 & 6 & 0 & -2.129551 & 0.103653 & 0.218953 \\
\hline 2 & 6 & 0 & -0.860 & 0.678341 & -0.209864 \\
\hline 3 & 6 & 0 & -0.932547 & -0.756273 & -0.363934 \\
\hline 4 & 1 & 0 & -1.213823 & -1.173707 & -1.328488 \\
\hline 5 & 6 & 0 & 0.2449 & 1.450693 & 0.099115 \\
\hline 6 & 6 & 0 & 0.2387 & -1.413945 & 0.362813 \\
\hline 7 & 1 & 0 & 0.256616 & -2.492104 & 0.187113 \\
\hline 8 & 6 & 0 & 1.594153 & 0.777792 & 0.064000 \\
\hline 9 & 6 & 0 & 1.533287 & -0.753113 & -0.179111 \\
\hline 10 & 1 & 0 & 1.610108 & -0.957400 & -1.253407 \\
\hline 11 & 1 & 0 & 2.283423 & 1.278406 & -0.625812 \\
\hline 12 & 1 & & & 2.448703 & 0.525004 \\
\hline 13 & 1 & & 0.147587 & -1.262801 & 1.446026 \\
\hline 14 & 1 & 0 & -0.398548 & 1.553754 & -1.082805 \\
\hline 15 & 1 & 0 & 1.993760 & 0.997685 & 1.067467 \\
\hline 16 & 1 & & 2.414505 & -1.208369 & 0.282545 \\
\hline 17 & 1 & & -2.366 & 0.180369 & 1.279164 \\
\hline 18 & 1 & 0 & -2.985824 & 0.112580 & -0.448644 \\
\hline
\end{tabular}

Energy: $\mathrm{HF}=-272.9984321$

Zero-Point Energy Correction: 0.159198

Imaginary Frequency: One imaginary frequency $-830.8671 \mathrm{~cm}^{-1}$ 

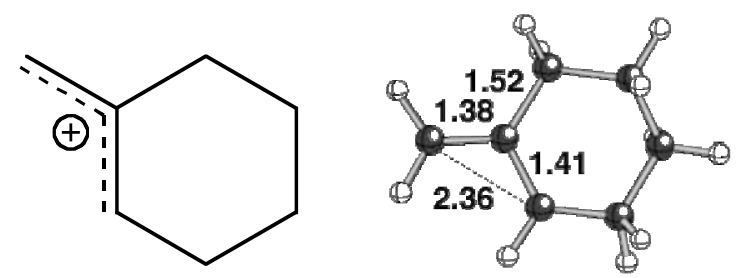

b3lyp/6-31G(d)

\section{Coordinates:}

Standard orientation:

\begin{tabular}{|c|c|c|c|c|c|}
\hline Center & Atomic & & Atomic & Coordinate & es (Angstro \\
\hline Number & Numbe & & Type & $\begin{array}{ll}X & Y\end{array}$ & l $\quad \mathrm{Z}$ \\
\hline 1 & 6 & 0 & -2.466946 & 0.060288 & -0.008119 \\
\hline 2 & 6 & 0 & -1.095826 & -0.019117 & 0.039121 \\
\hline 3 & 6 & 0 & -0.408787 & 1.209469 & -0.047349 \\
\hline 4 & 6 & 0 & -0.324601 & -1.320109 & 0.169364 \\
\hline 5 & 6 & 0 & 1.048417 & 1.329118 & -0.044902 \\
\hline 6 & 1 & 0 & 1.335241 & 2.183768 & 0.591438 \\
\hline 7 & 6 & 0 & 1.116336 & -1.184201 & -0.351501 \\
\hline 8 & 6 & 0 & 1.816627 & 0.034963 & 0.264290 \\
\hline 9 & 1 & 0 & 1.889123 & -0.084841 & 1.352172 \\
\hline 10 & 1 & 0 & 1.671800 & -2.096291 & -0.116243 \\
\hline 11 & 1 & 0 & -0.860412 & -2.115140 & -0.359600 \\
\hline 12 & 1 & 0 & 1.287545 & 1.715126 & -1.061173 \\
\hline 13 & 1 & 0 & 26 & -1.095910 & -1.445968 \\
\hline 14 & 1 & 0 & 2.840039 & 0.126306 & -0.109530 \\
\hline 15 & 1 & 0 & -0.312383 & -1.606042 & 1.229992 \\
\hline 16 & 1 & 0 & -0.994212 & 2.124003 & -0.151538 \\
\hline 17 & 1 & 0 & -3.093621 & -0.828031 & 0.044059 \\
\hline 18 & 1 & 0 & -2.981172 & 1.014583 & -0.099031 \\
\hline
\end{tabular}

Energy: $\mathrm{HF}=-273.0681797$

Zero-Point Energy Correction: $\mathbf{0 . 1 6 2 7 4 5}$

Imaginary Frequency: Minimum (No imaginary frequencies) 


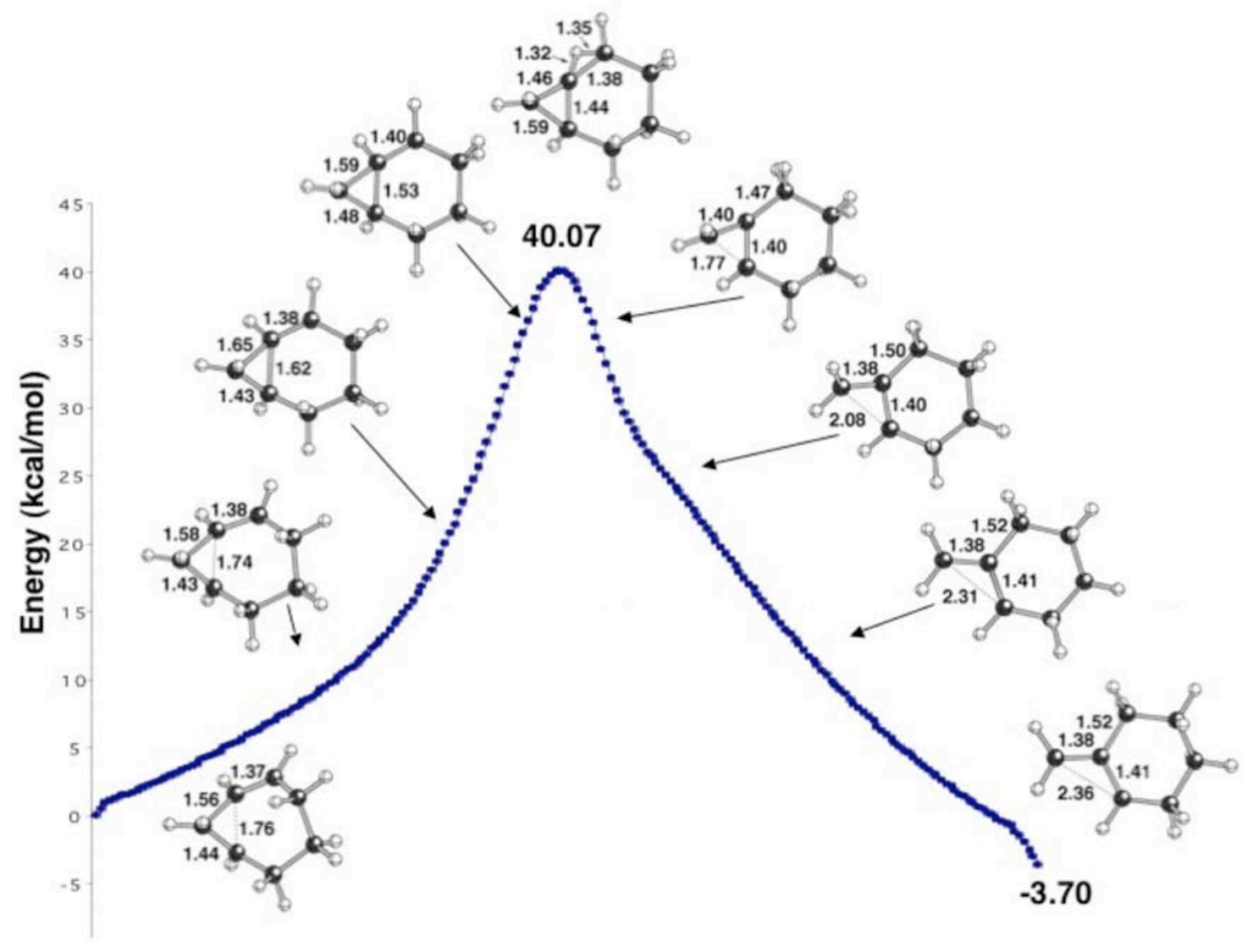



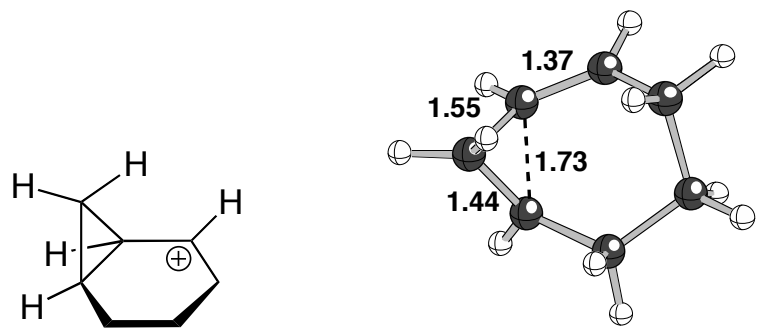

MP2/6-31G(d)

\section{Coordinates:}

Standard orientation:

\begin{tabular}{|c|c|c|c|c|}
\hline \multirow{2}{*}{$\begin{array}{l}\text { Center } \\
\text { Number }\end{array}$} & \multirow{2}{*}{$\begin{array}{l}\text { Atomic } \\
\text { Number }\end{array}$} & \multirow{2}{*}{$\begin{array}{r}\text { Atomic } \\
\text { Type }\end{array}$} & \multicolumn{2}{|c|}{ Coordinates (Angstroms) } \\
\hline & & & $\begin{array}{ll}X & Y\end{array}$ & $\mathrm{Z}$ \\
\hline 1 & 6 & 1.679034 & 0.181344 & 0.654832 \\
\hline 2 & 6 & 1.117813 & -0.838790 & -0.373960 \\
\hline 3 & 6 & 0.903226 & 0.876041 & -0.339505 \\
\hline 4 & 1 & 1.422068 & 1.226974 & -1.230434 \\
\hline 5 & 6 & -0.121879 & -1.419409 & -0.279238 \\
\hline 6 & 6 & -0.464845 & 1.440276 & -0.042541 \\
\hline 7 & 1 & -0.624076 & 2.294770 & -0.707781 \\
\hline 8 & 6 & -1.234560 & -0.872756 & 0.520294 \\
\hline 9 & 6 & -1.625302 & 0.450993 & -0.196029 \\
\hline 10 & 1 & -1.829031 & 0.261157 & -1.255380 \\
\hline 11 & 1 & -2.075105 & -1.568665 & 0.546157 \\
\hline 12 & 1 & -0.330975 & -2.256678 & -0.947000 \\
\hline 13 & 1 & -0.456255 & 1.815715 & 0.986989 \\
\hline 14 & 1 & -0.935138 & -0.643856 & 1.549776 \\
\hline 15 & 1 & -2.540211 & 0.854908 & 0.247272 \\
\hline 16 & 1 & 1.258395 & 0.089642 & 1.653148 \\
\hline 17 & 1 & 2.764332 & 0.213314 & 0.610115 \\
\hline 18 & 1 & 1.825074 & -1.193476 & -1.115983 \\
\hline
\end{tabular}

Energy: MP2=-272.0859738

Zero-Point Energy Correction: 0.167604

Imaginary Frequency: Minimum (No imaginary frequencies) 


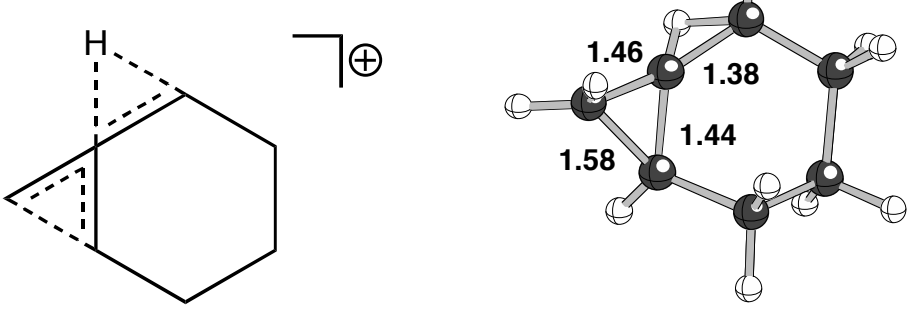

MP2/6-31G(d)

Coordinates:

Standard orientation:

\begin{tabular}{|c|c|c|c|c|c|}
\hline \multirow{2}{*}{$\begin{array}{l}\text { Center } \\
\text { Number }\end{array}$} & \multirow{2}{*}{$\begin{array}{l}\text { Atomic } \\
\text { Number }\end{array}$} & \multirow{2}{*}{\multicolumn{2}{|c|}{$\begin{array}{l}\text { Atomic } \\
\text { Type }\end{array}$}} & \multicolumn{2}{|c|}{ Coordinates (Angstron } \\
\hline & & & & X $\quad \mathrm{Y}$ & $\mathrm{Z}$ \\
\hline 1 & 6 & 0 & -2.118121 & 0.110590 & 0.210720 \\
\hline 2 & 6 & 0 & -0.847968 & 0.681831 & -0.218086 \\
\hline 3 & 6 & 0 & -0.929771 & -0.752156 & -0.362361 \\
\hline 4 & 1 & 0 & -1.204601 & -1.176400 & -1.326288 \\
\hline 5 & 6 & 0 & 0.256907 & 1.438750 & 0.110765 \\
\hline 6 & 6 & 0 & 0.227611 & -1.392520 & 0.382657 \\
\hline 7 & 1 & 0 & 0.234579 & -2.476798 & 0.249841 \\
\hline 8 & 6 & 0 & 1.596397 & 0.759913 & 0.060901 \\
\hline 9 & 6 & 0 & 1.510837 & -0.759240 & -0.194769 \\
\hline 10 & 1 & 0 & 1.542767 & -0.956066 & -1.271887 \\
\hline 11 & 1 & 0 & 2.286036 & 1.260262 & -0.628193 \\
\hline 12 & 1 & 0 & 0.142471 & 2.437848 & 0.537313 \\
\hline 13 & 1 & 0 & 0.142617 & -1.194830 & 1.458802 \\
\hline 14 & 1 & 0 & -0.398030 & 1.544289 & -1.069267 \\
\hline 15 & 1 & 0 & 1.999781 & 0.959565 & 1.065762 \\
\hline 16 & 1 & 0 & 2.400814 & -1.229878 & 0.232739 \\
\hline 17 & 1 & 0 & -2.349630 & 0.183264 & 1.271694 \\
\hline 18 & 1 & 0 & -2.972154 & 0.125740 & -0.459483 \\
\hline
\end{tabular}

Energy: MP2 $=-272.0187501$

Zero-Point Energy Correction: 0.162478

Imaginary Frequency: One imaginary frequency $-635.2341 \mathrm{~cm}^{-1}$ 

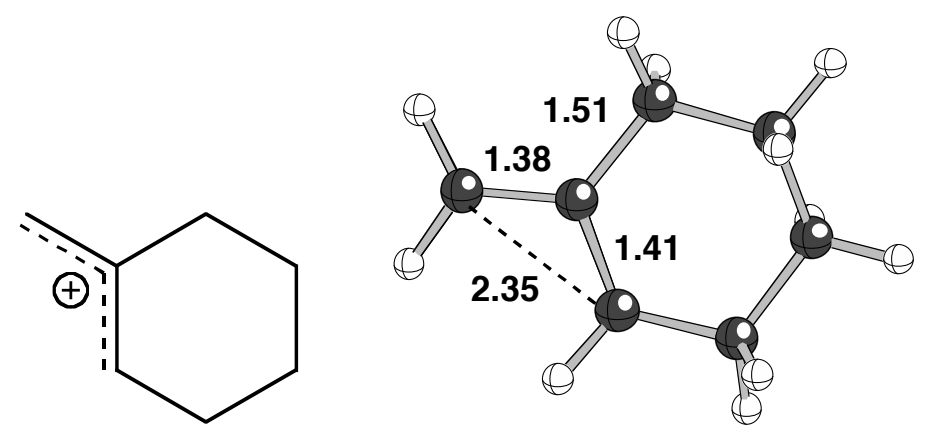

MP2/6-31G(d)

\section{Coordinates:}

Standard orientation:

\begin{tabular}{|c|c|c|c|c|}
\hline \multirow{2}{*}{$\begin{array}{l}\text { Center } \\
\text { Number }\end{array}$} & \multirow{2}{*}{$\begin{array}{l}\text { Atomic } \\
\text { Number }\end{array}$} & \multirow{2}{*}{$\begin{array}{r}\text { Atomic } \\
\text { Type }\end{array}$} & \multicolumn{2}{|c|}{ Coordinates (Angstrol } \\
\hline & & & X $\quad Y$ & I $\quad Z$ \\
\hline 1 & 6 & -2.457473 & 0.064944 & 0.003764 \\
\hline 2 & 6 & -1.082905 & -0.024258 & 0.034425 \\
\hline 3 & 6 & -0.405397 & 1.203592 & -0.053276 \\
\hline 4 & 6 & -0.320003 & -1.322076 & 0.157764 \\
\hline 5 & 6 & 1.0515 & 1.324846 & -0.054688 \\
\hline 6 & 1 & 50 & 2.186383 & 0.566588 \\
\hline 7 & 6 & 76 & -1.170159 & -0.359147 \\
\hline 8 & 6 & 1.7938 & 0.033352 & 0.290458 \\
\hline 9 & 1 & 1.813759 & -0.093146 & 1.378695 \\
\hline 10 & 1 & 1.672329 & -2.084855 & -0.145229 \\
\hline 11 & 1 & -0.855092 & -2.108462 & -0.384251 \\
\hline 12 & 1 & 0 & 1.6679 & -1.082696 \\
\hline 13 & 1 & 103703 & -1.052696 & -1.449785 \\
\hline 14 & 1 & 2.8334 & 0.117140 & -0.036681 \\
\hline 15 & 1 & -0.307870 & -1.616591 & 1.214431 \\
\hline 16 & 1 & -0.998357 & 2.115225 & -0.159791 \\
\hline 17 & 1 & -3.090 & -0.817852 & 0.068255 \\
\hline 18 & 1 & -2.960877 & 1.025478 & -0.085329 \\
\hline
\end{tabular}

Energy: MP2 $=-272.0828657$

Zero-Point Energy Correction: $\mathbf{0 . 1 6 5 9 2 7}$

Imaginary Frequency: Minimum (No imaginary frequencies) 


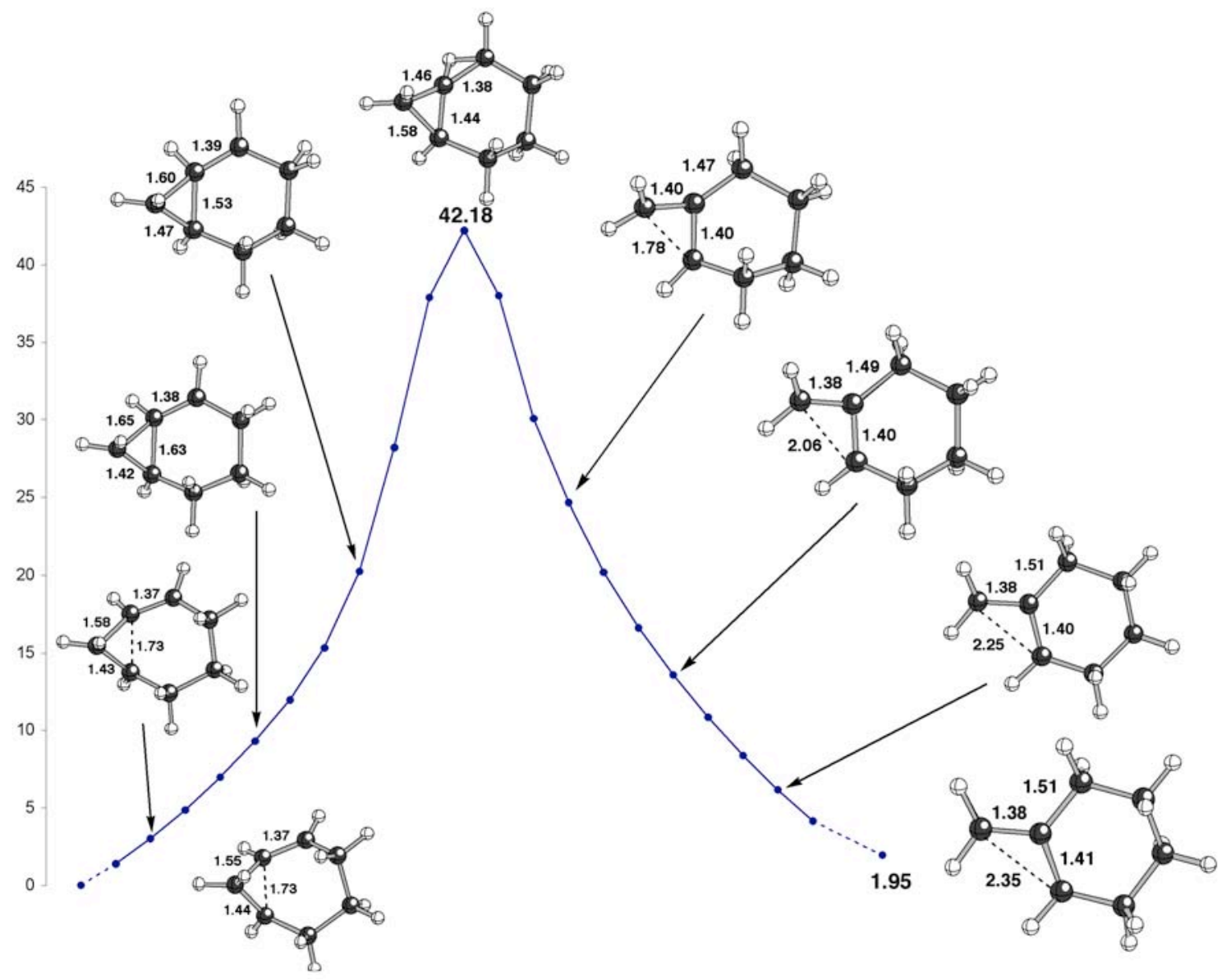



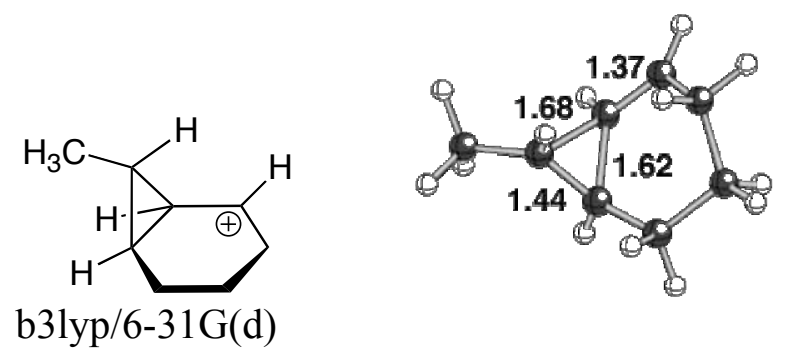

\section{Coordinates:}

Standard orientation:

\begin{tabular}{|c|c|c|c|c|c|}
\hline \multirow{2}{*}{$\begin{array}{l}\text { Center } \\
\text { Number }\end{array}$} & \multirow{2}{*}{$\begin{array}{l}\text { Atomic } \\
\text { Number }\end{array}$} & \multirow{2}{*}{\multicolumn{2}{|c|}{$\begin{array}{l}\text { Atomic } \\
\text { Type }\end{array}$}} & \multicolumn{2}{|c|}{ Coordinates (Angstro } \\
\hline & & & & X $\quad Y$ & Z \\
\hline 1 & 6 & 0 & 1.329729 & 0.118644 & 0.444279 \\
\hline 2 & 6 & 0 & 469861 & -0.794835 & -0.670118 \\
\hline 3 & 6 & 0 & 0.483097 & 0.815637 & -0.483231 \\
\hline 4 & 1 & 0 & 0.977673 & 1.240490 & -1.356218 \\
\hline 5 & 6 & 0 & -0.665288 & -1.454531 & -0.270390 \\
\hline 6 & 6 & 0 & -0.828611 & 1.472376 & -0.035584 \\
\hline 7 & 1 & 0 & -1.020655 & 2.321021 & -0.699398 \\
\hline 8 & 6 & 0 & 01933 & -0.838360 & 0.593558 \\
\hline 9 & 6 & 0 & -2.0 & 0.550152 & -0.028062 \\
\hline 10 & 1 & 0 & -2.431731 & 0.399842 & -1.04520 \\
\hline 11 & 1 & 0 & -2.579754 & -1.483183 & $0.66937 c$ \\
\hline 12 & 1 & 0 & -0.862076 & -2.432444 & -0.71066 \\
\hline 13 & 1 & 0 & -0.674832 & 1.881334 & 0.969958 \\
\hline 14 & 1 & & -1.320940 & -0.682 & 1.614132 \\
\hline 15 & 1 & 0 & -2.864294 & 1.001076 & 0.552355 \\
\hline 16 & 6 & 0 & 2.820343 & -0.016074 & 0.304138 \\
\hline 17 & 1 & 0 & 3.286010 & 0.764296 & 0.920494 \\
\hline 18 & 1 & 0 & 3.174732 & -0.982796 & 0.673140 \\
\hline 19 & 1 & 0 & 3.154598 & 0.124543 & -0.72776 \\
\hline 20 & 1 & & 0.928673 & -0.049475 & 1.44245 \\
\hline 21 & 1 & 0 & 1.114882 & -1.220759 & -1.43018 \\
\hline
\end{tabular}

Energy: $\mathrm{HF}=-312.3850793$

\section{Zero-Point Energy Correction: 0.191853}

Imaginary Frequency: Minimum (No imaginary frequencies) 


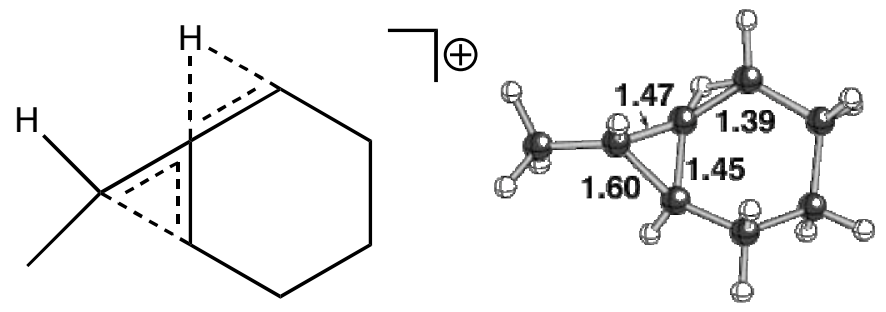

b3lyp/6-31G(d)

\section{Coordinates:}

Standard orientation:

\begin{tabular}{|c|c|c|c|c|}
\hline \multirow{2}{*}{$\begin{array}{l}\text { Center } \\
\text { Number }\end{array}$} & \multirow{2}{*}{$\begin{array}{l}\text { Atomic } \\
\text { Number }\end{array}$} & \multirow{2}{*}{$\begin{array}{r}\text { Atomic } \\
\text { Type }\end{array}$} & \multicolumn{2}{|c|}{ Coordinates (Angstror } \\
\hline & & & X $\quad Y$ & $Y \quad Z$ \\
\hline 1 & 6 & -1.633758 & 0.038008 & 0.465615 \\
\hline 2 & 6 & -0.419755 & 0.654127 & -0.082450 \\
\hline 3 & 6 & -0.465816 & -0.782969 & -0.231879 \\
\hline 4 & 1 & -0.830290 & -1.201616 & -1.168268 \\
\hline 5 & 6 & 0.693455 & 1.450930 & 0.125897 \\
\hline 6 & 6 & 787597 & -1.415669 & 0.368372 \\
\hline 7 & 1 & 57 & -2.491251 & 0.178443 \\
\hline 8 & 6 & 2.050758 & 0.812861 & -0.026723 \\
\hline 9 & 6 & 2.008 & -0.715705 & -0.283445 \\
\hline 10 & 1 & 1.988527 & -0.905860 & -1.362965 \\
\hline 11 & 1 & 03 & 1.338436 & -0.764587 \\
\hline 12 & 1 & 0 . & 2.449806 & 0.554380 \\
\hline 13 & 1 & 0.797743 & -1.278460 & 1.457475 \\
\hline 14 & 1 & -0.056960 & 1.531293 & -0.994811 \\
\hline 15 & 1 & 2.527050 & 1.031851 & 0.943100 \\
\hline 16 & 1 & 2.94 & -1.1513 & 0.086982 \\
\hline 17 & 6 & -2.983 & 0.036883 & -0.222842 \\
\hline 18 & 1 & -3.561936 & -0.830839 & 0.106647 \\
\hline 19 & 1 & -3.541704 & 0.939623 & 0.045278 \\
\hline 20 & 1 & -2.888549 & -0.000968 & -1.312070 \\
\hline 21 & 1 & -1.674483 & 0.098569 & 1.555127 \\
\hline
\end{tabular}

Energy: $\mathrm{HF}=-312.3193874$

Imaginary Frequency: One imaginary frequency $-837.8942 \mathrm{~cm}^{-1}$ 

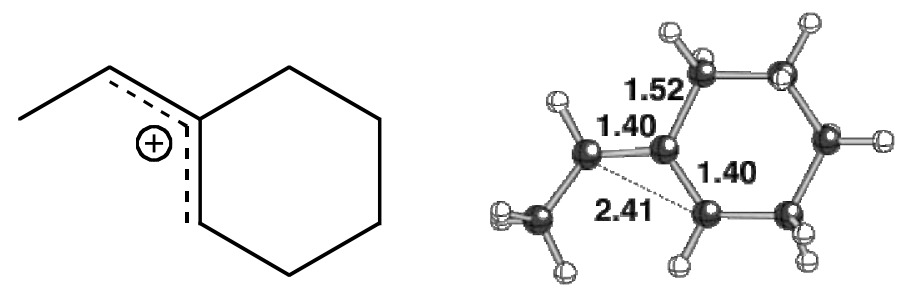

b3lyp/6-31G(d)

\section{Coordinates:}

Standard orientation:

\begin{tabular}{|c|c|c|c|c|c|}
\hline \multirow{2}{*}{$\begin{array}{l}\text { Center } \\
\text { Number }\end{array}$} & \multirow{2}{*}{\multicolumn{2}{|c|}{$\begin{array}{l}\text { Atomic } \\
\text { Number }\end{array}$}} & \multirow{2}{*}{$\begin{array}{l}\text { Atomic } \\
\text { Type }\end{array}$} & \multicolumn{2}{|c|}{ Coordinates (Angstrom } \\
\hline & & & & X $\quad \mathrm{Y}$ & $\mathrm{Z}$ \\
\hline 1 & 6 & 0 & 1.943633 & -0.562531 & -0.040022 \\
\hline 2 & 6 & 0 & 0.577870 & -0.279424 & -0.054111 \\
\hline 3 & 6 & 0 & 0.152554 & 1.045747 & 0.034485 \\
\hline 4 & 1 & 0 & 0.892679 & 1.837493 & 0.130103 \\
\hline 5 & 6 & 0 & -0.444017 & -1.403831 & -0.161154 \\
\hline 6 & 6 & 0 & -1.259531 & 1.458649 & 0.043209 \\
\hline 7 & 1 & 0 & -1.371938 & 2.338290 & -0.611649 \\
\hline 8 & 6 & 0 & -1.824927 & -0.984082 & 0.365808 \\
\hline 9 & 6 & 0 & -2.271245 & 0.343994 & -0.259138 \\
\hline 10 & 1 & 0 & -2.366785 & 0.230502 & -1.346005 \\
\hline 11 & 1 & 0 & -2.551192 & -1.771119 & 0.143728 \\
\hline 12 & 1 & 0 & -0.075217 & -2.282042 & 0.380258 \\
\hline 13 & 1 & 0 & -1.420874 & 1.891278 & 1.052630 \\
\hline 14 & 1 & 0 & -0.524126 & -1.701200 & -1.215745 \\
\hline 15 & 1 & 0 & -1.789170 & -0.887753 & 1.459207 \\
\hline 16 & 1 & 0 & -3.256443 & 0.638896 & 0.112744 \\
\hline 17 & 6 & 0 & 3.108026 & 0.337318 & 0.041074 \\
\hline 18 & 1 & 0 & 3.723904 & 0.038167 & 0.903695 \\
\hline 19 & 1 & 0 & 3.752636 & 0.151382 & -0.832682 \\
\hline 20 & 1 & 0 & 2.888522 & 1.402772 & 0.105174 \\
\hline 21 & 1 & 0 & 2.203834 & -1.621711 & -0.102359 \\
\hline
\end{tabular}

Energy: $\mathrm{HF}=-312.399478$

Zero-Point Energy Correction: 0.190977

Imaginary Frequency: Minimum (No imaginary frequencies) 


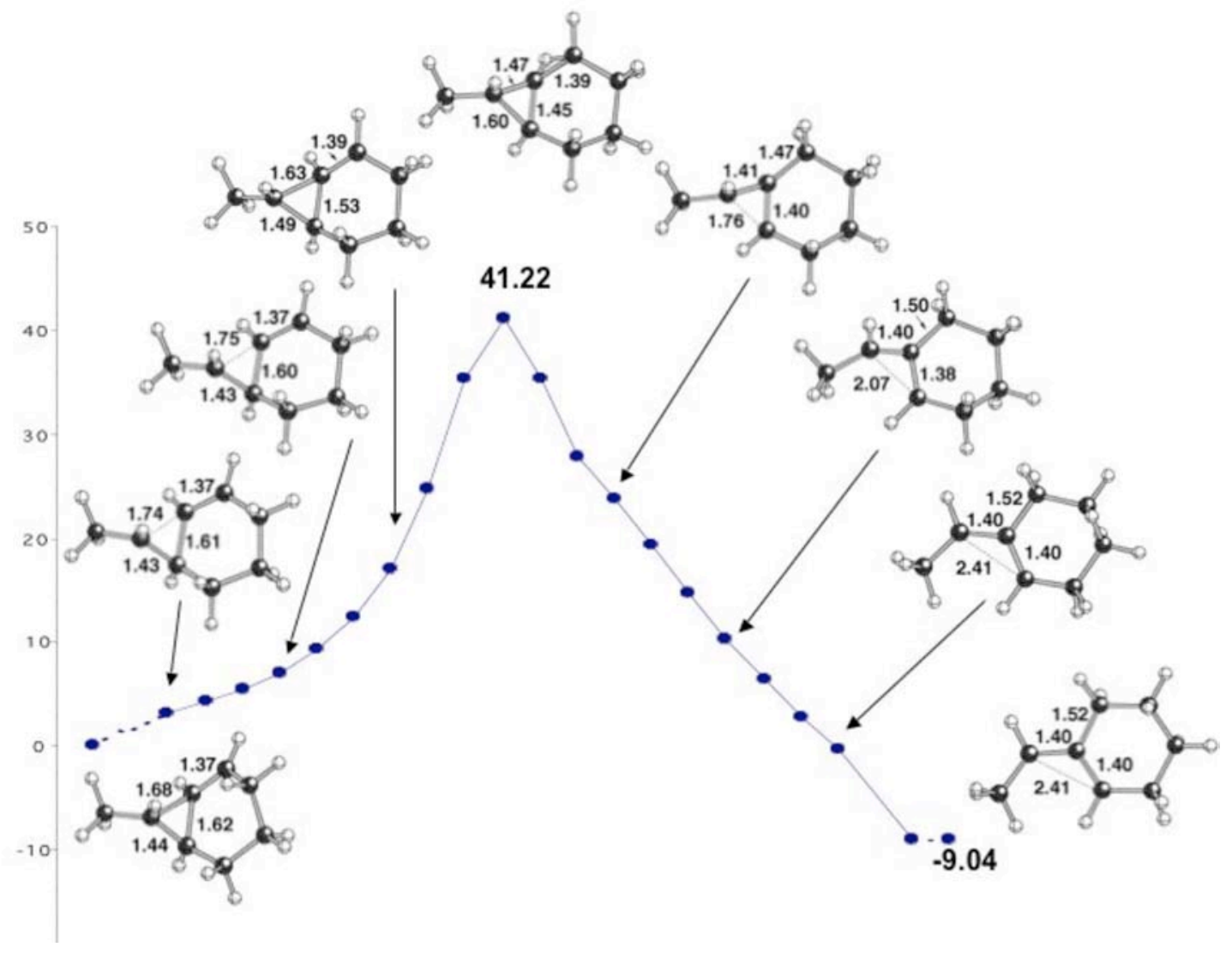




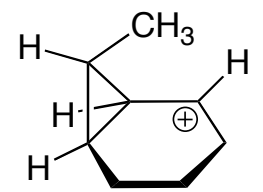

b3lyp/6-31G(d)

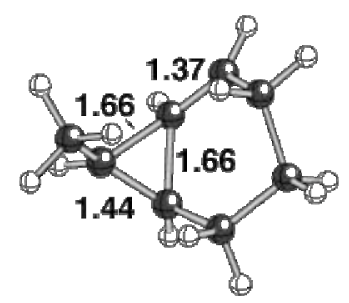

\section{Coordinates:}

Standard orientation:

\begin{tabular}{|c|c|c|c|c|c|}
\hline \multirow{2}{*}{$\begin{array}{l}\text { Center } \\
\text { Number }\end{array}$} & \multirow{2}{*}{\multicolumn{2}{|c|}{$\begin{array}{l}\text { Atomic } \\
\text { Number }\end{array}$}} & \multirow{2}{*}{$\begin{array}{l}\text { Atomic } \\
\text { Type }\end{array}$} & \multicolumn{2}{|c|}{ Coordinates (Angstrom } \\
\hline & & & & X $\quad Y$ & Z \\
\hline 1 & 6 & 0 & -1.929130 & -0.049404 & 1.192895 \\
\hline 2 & 1 & 0 & -2.626335 & -0.875083 & 1.385815 \\
\hline 3 & 1 & 0 & -2.434847 & 0.879209 & 1.470147 \\
\hline 4 & 1 & 0 & -1.065119 & -0.193014 & 1.843029 \\
\hline 5 & 6 & 0 & -1.612748 & -0.068674 & -0.278944 \\
\hline 6 & 1 & 0 & -2.482058 & 0.106117 & -0.911415 \\
\hline 7 & 6 & 0 & -0.451973 & 0.931816 & -0.912213 \\
\hline 8 & 6 & 0 & -0.507995 & -0.731275 & -0.915181 \\
\hline 9 & 1 & 0 & -0.645541 & -1.015234 & -1.957430 \\
\hline 10 & 6 & 0 & 0.627032 & 1.461688 & -0.251528 \\
\hline 11 & 6 & 0 & 0.580750 & -1.466405 & -0.140899 \\
\hline 12 & 1 & 0 & 0.864311 & -2.342160 & -0.734356 \\
\hline 13 & 6 & 0 & 1.448672 & 0.749492 & 0.758561 \\
\hline 14 & 6 & 0 & 1.838179 & -0.638025 & 0.160778 \\
\hline 15 & 1 & 0 & 2.427242 & -0.484894 & -0.750047 \\
\hline 16 & 1 & 0 & 2.333487 & 1.335726 & 1.015276 \\
\hline 17 & 1 & 0 & 0.982622 & 2.429348 & -0.607367 \\
\hline 18 & 1 & 0 & 0.159371 & -1.842251 & 0.797686 \\
\hline 19 & 1 & 0 & 0.880001 & 0.588106 & 1.684446 \\
\hline 20 & 1 & 0 & 2.477869 & -1.166656 & 0.873352 \\
\hline 21 & 1 & 0 & -0.827727 & 1.445511 & -1.789948 \\
\hline
\end{tabular}

Energy: $\mathrm{HF}=-312.3795742$ 


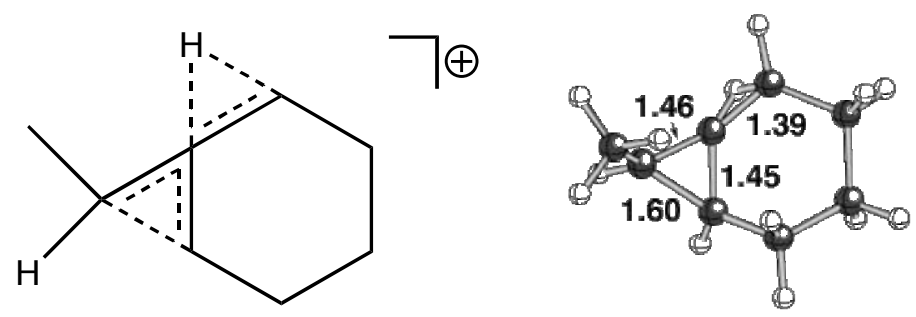

b3lyp/6-31G(d)

Coordinates:

Standard orientation:

\begin{tabular}{|c|c|c|c|c|}
\hline \multirow{2}{*}{$\begin{array}{l}\text { Center } \\
\text { Number }\end{array}$} & \multirow{2}{*}{$\begin{array}{l}\text { Atomic } \\
\text { Number }\end{array}$} & \multirow{2}{*}{$\begin{array}{r}\text { Atomic } \\
\quad \text { Type }\end{array}$} & \multicolumn{2}{|c|}{ Coordinates (Angstror } \\
\hline & & & X $\quad Y$ & $\mathrm{Z}$ \\
\hline 1 & 6 & -2.524420 & 0.104723 & 0.791412 \\
\hline 2 & 1 & -3.180725 & -0.763570 & 0.908835 \\
\hline 3 & 1 & -3.155125 & 0.998892 & 0.751093 \\
\hline 4 & 1 & -1.882838 & 0.174703 & 1.674751 \\
\hline 5 & 6 & -1.728181 & -0.042190 & -0.484990 \\
\hline 6 & 1 & -2.315354 & -0.148191 & -1.394319 \\
\hline 7 & 6 & -0.414518 & 0.590944 & -0.626290 \\
\hline 8 & 6 & -0.361462 & -0.853280 & -0.686429 \\
\hline 9 & 1 & -0.377246 & -1.342927 & -1.657416 \\
\hline 10 & 6 & 523884 & 1.440594 & -0.059857 \\
\hline 11 & 6 & 0.623120 & -1.397951 & 0.343859 \\
\hline 12 & 1 & 0.751413 & -2.477263 & 0.232751 \\
\hline 13 & 6 & 1.868026 & 0.869225 & 0.302059 \\
\hline 14 & 6 & 1.971672 & -0.665615 & 0.118135 \\
\hline 15 & 1 & 2.330432 & -0.890279 & -0.893006 \\
\hline 16 & 1 & 2.686042 & 1.403824 & -0.195539 \\
\hline 17 & 1 & 0.247371 & 2.450607 & 0.247553 \\
\hline 18 & 1 & 0.254956 & -1.220166 & 1.362254 \\
\hline 19 & 1 & 0.194464 & 1.436683 & -1.396100 \\
\hline 20 & 1 & 1.9644 & 1.143689 & 1.365915 \\
\hline 21 & 1 & 2.733410 & -1.044704 & 0.805837 \\
\hline
\end{tabular}

Energy: $\mathrm{HF}=-312.3204561$

Zero-Point Energy Correction: $\mathbf{0 . 1 8 7 3 8 4}$

Imaginary Frequency: One imaginary frequency $\mathbf{- 8 5 3 . 0 3 9 0 ~ \mathrm { cm } ^ { - 1 }}$ 

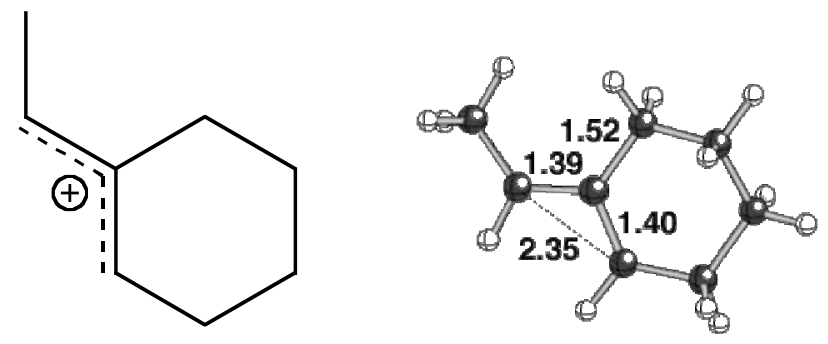

b3lyp/6-31G(d)

\section{Coordinates:}

Standard orientation:

\begin{tabular}{|c|c|c|c|c|c|}
\hline \multirow{2}{*}{$\begin{array}{l}\text { Center } \\
\text { Number }\end{array}$} & \multirow{2}{*}{$\begin{array}{l}\text { Atomic } \\
\text { Number }\end{array}$} & \multirow{2}{*}{\multicolumn{2}{|c|}{$\begin{array}{l}\text { Atomic } \\
\text { Type }\end{array}$}} & \multicolumn{2}{|c|}{ Coordinates (Angstroms) } \\
\hline & & & & $\begin{array}{ll}X & Y\end{array}$ & I $\quad \mathrm{Z}$ \\
\hline 1 & 6 & 0 & -3.088718 & -0.284764 & 0.080960 \\
\hline 2 & 1 & 0 & -3.733138 & -0.158289 & -0.804684 \\
\hline 3 & 1 & 0 & -3.715412 & 0.053804 & 0.921614 \\
\hline 4 & 1 & 0 & -2.838775 & -1.337880 & 0.207795 \\
\hline 5 & 6 & 0 & -1.928241 & 0.608286 & -0.041507 \\
\hline 6 & 1 & 0 & -2.156350 & 1.670050 & -0.145892 \\
\hline 7 & 6 & 0 & -0.583582 & 0.246156 & -0.026011 \\
\hline 8 & 6 & 0 & 0.318409 & 1.309882 & -0.086768 \\
\hline 9 & 1 & 0 & -0.076616 & 2.320213 & -0.198275 \\
\hline 10 & 6 & 0 & -0.086171 & -1.184744 & 0.084069 \\
\hline 11 & 6 & 0 & 1.780022 & 1.158645 & -0.024095 \\
\hline 12 & 1 & 0 & 2.190023 & 1.940140 & 0.635325 \\
\hline 13 & 6 & 0 & 1.386613 & -1.316857 & -0.341489 \\
\hline 14 & 6 & 0 & 2.262621 & -0.254842 & 0.334292 \\
\hline 15 & 1 & 0 & 2.229706 & -0.380402 & 1.423603 \\
\hline 16 & 1 & 0 & 1.743301 & -2.319914 & -0.090876 \\
\hline 17 & 1 & 0 & -0.714728 & -1.840797 & -0.527409 \\
\hline 18 & 1 & 0 & 2.143143 & 1.469338 & -1.025650 \\
\hline 19 & 1 & 0 & -0.208486 & -1.519528 & 1.123373 \\
\hline 20 & 1 & 0 & 1.462696 & -1.220036 & -1.432634 \\
\hline 21 & 1 & 0 & 3.308913 & -0.367270 & 0.037008 \\
\hline
\end{tabular}

Energy: $\mathrm{HF}=-312.4043057$

Zero-Point Energy Correction: 0.191076

Imaginary Frequency: Minimum (No imaginary frequencies) 


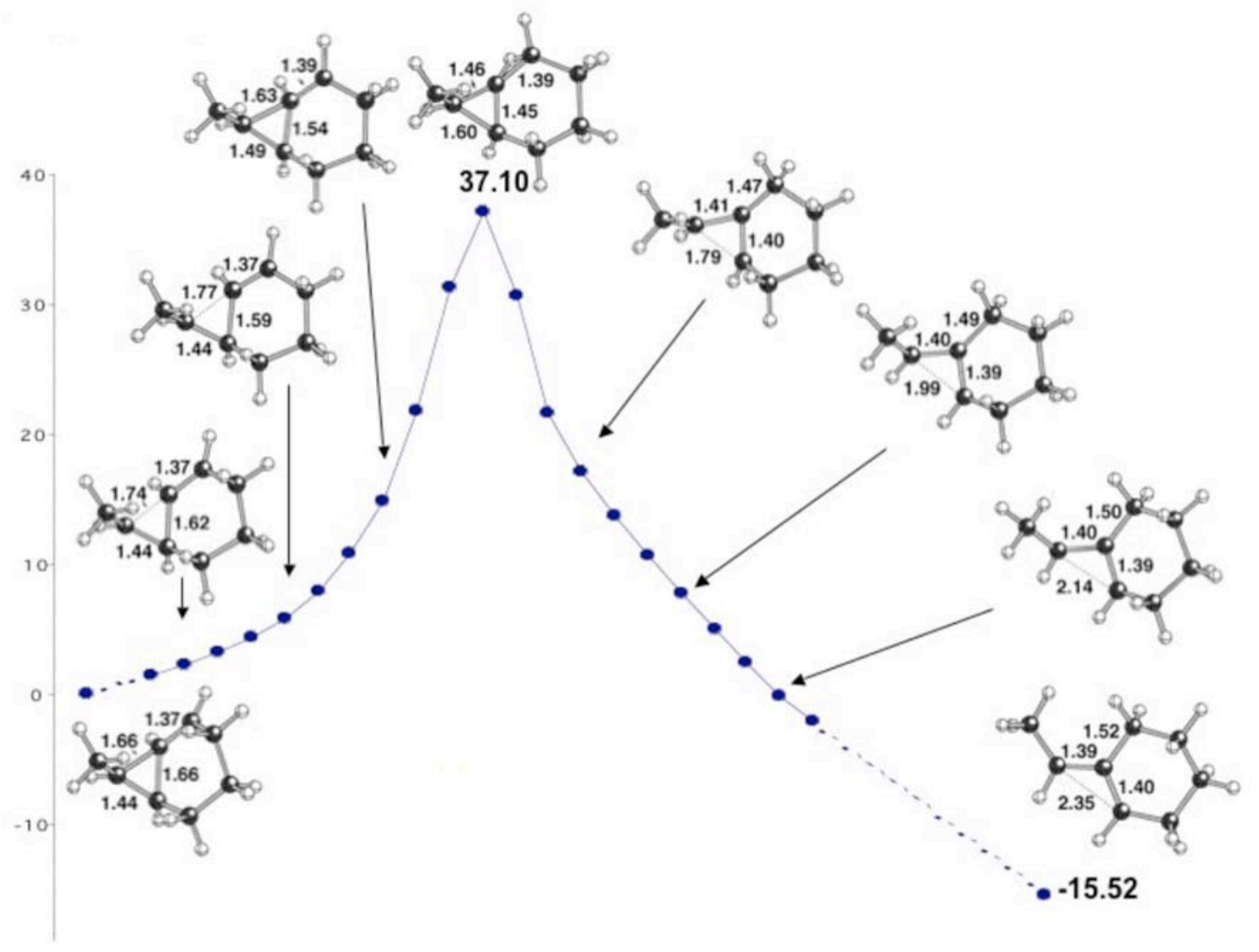




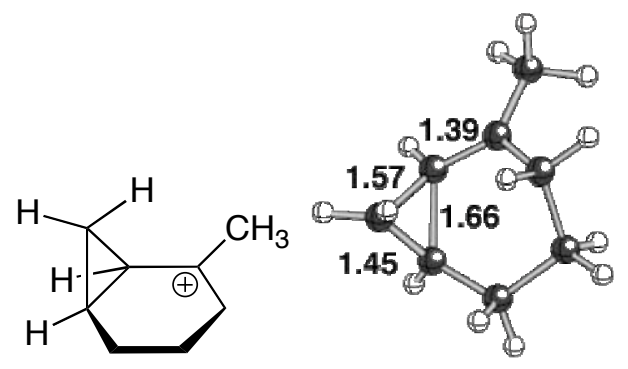

b3lyp/6-31G(d)

\section{Coordinates:}

Standard orientation:

\begin{tabular}{|c|c|c|c|c|}
\hline \multirow{2}{*}{$\begin{array}{l}\text { Center } \\
\text { Number }\end{array}$} & \multirow{2}{*}{$\begin{array}{l}\text { Atomic } \\
\text { Number }\end{array}$} & \multirow{2}{*}{$\begin{array}{r}\text { Atomic } \\
\text { Type }\end{array}$} & \multicolumn{2}{|c|}{ Coordinates (Angstrom } \\
\hline & & & $\begin{array}{ll}\mathrm{X} & \mathrm{Y}\end{array}$ & Z $\quad \mathrm{Z}$ \\
\hline 1 & 6 & 1.078138 & -1.526565 & 0.672411 \\
\hline 2 & 6 & -0.188043 & -1.200857 & -0.204930 \\
\hline 3 & 6 & 1.368592 & -0.689908 & -0.469025 \\
\hline 4 & 1 & 1.719678 & -1.185444 & -1.371148 \\
\hline 5 & 6 & -1.081669 & -0.175762 & 0.101198 \\
\hline 6 & 6 & 1.682419 & 0.794757 & -0.316426 \\
\hline 7 & 1 & 2.278092 & 1.109467 & -1.178723 \\
\hline 8 & 6 & -0.605118 & 1.070781 & 0.763027 \\
\hline 9 & 6 & 0.454554 & 1.708698 & -0.188828 \\
\hline 10 & 1 & 0.001910 & 1.882764 & -1.171920 \\
\hline 11 & 1 & -1.434244 & 1.759798 & 0.940650 \\
\hline 12 & 1 & 2.313369 & 0.914161 & 0.572633 \\
\hline 13 & 1 & -0.529999 & -2.017740 & -0.830700 \\
\hline 14 & 1 & -0.130266 & 0.855307 & 1.729284 \\
\hline 15 & 1 & 0.741927 & 2.686630 & 0.208179 \\
\hline 16 & 1 & 1.077650 & -1.083945 & 1.664927 \\
\hline 17 & 1 & 1.310807 & -2.587521 & 0.643383 \\
\hline 18 & 6 & -2.495291 & -0.249031 & -0.344522 \\
\hline 19 & 1 & -2.716763 & -1.121384 & +-0.961666 \\
\hline 20 & 1 & -3.135032 & -0.275170 & 0.552125 \\
\hline 21 & 1 & -2.778618 & 0.670398 & -0.874457 \\
\hline
\end{tabular}

Energy: $H F=-312.394912$

Zero-Point Energy Correction: 0.192151

Imaginary Frequency: Minimum (No imaginary frequencies) 

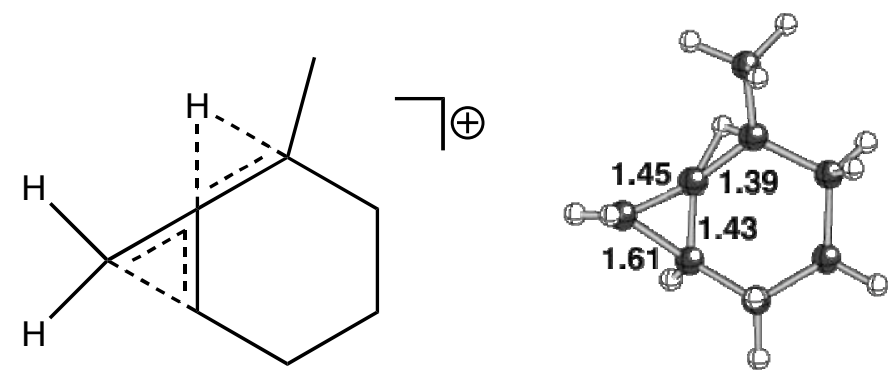

b3lyp/6-31G(d)

\section{Coordinates:}

Standard orientation:

\begin{tabular}{|c|c|c|c|c|}
\hline \multirow{2}{*}{$\begin{array}{l}\text { Center } \\
\text { Number }\end{array}$} & \multirow{2}{*}{$\begin{array}{l}\text { Atomic } \\
\text { Numbe }\end{array}$} & \multirow{2}{*}{$\begin{array}{c}\text { Atomic } \\
\text { Type }\end{array}$} & \multicolumn{2}{|c|}{ Coordinates (Angstror } \\
\hline & & & X $\quad Y$ & $\mathrm{Z}$ \\
\hline 1 & 6 & -0.366153 & 2.130265 & 0.144382 \\
\hline 2 & 6 & 0.165 & 0.877570 & -0.347515 \\
\hline 3 & 6 & -1.267313 & 0.866633 & -0.273806 \\
\hline 4 & 1 & -1.857836 & 1.142040 & -1.144683 \\
\hline 5 & 6 & 1.079679 & -0.159896 & -0.190375 \\
\hline 6 & 6 & -1.741226 & -0.336546 & 0.537672 \\
\hline 7 & 1 & -2.83 & -0.412964 & 0.528354 \\
\hline 8 & 6 & 0.454983 & -1.553689 & -0.101086 \\
\hline 9 & 6 & -1.095635 & -1.581125 & -0.121986 \\
\hline 10 & 1 & -1.451176 & -1.653714 & -1.156686 \\
\hline 11 & 1 & 41 & -2.223210 & -0.858823 \\
\hline 12 & 1 & -1.429616 & -0.240879 & 1.585139 \\
\hline 13 & 1 & 0.947283 & 0.341915 & -1.370340 \\
\hline 14 & 1 & 0.824690 & -1.935277 & 0.861176 \\
\hline 15 & 1 & -1.429119 & -2.494084 & 0.380605 \\
\hline 16 & 1 & -0.11 & 2.408955 & 1.167360 \\
\hline 17 & 1 & -0.551200 & 2.961794 & -0.528965 \\
\hline 18 & 6 & 2.507633 & 0.057374 & 0.250788 \\
\hline 19 & 1 & 2.852324 & 1.073835 & 0.045364 \\
\hline 20 & 1 & 2.562546 & -0.113098 & 1.332851 \\
\hline 21 & 1 & 3.180148 & -0.658835 & -0.229797 \\
\hline
\end{tabular}

Energy: $\mathrm{HF}=-312.325232$

Zero-Point Energy Correction: 0.187403

Imaginary Frequency: One imaginary frequency $\mathbf{- 8 3 3 . 6 8 6 8} \mathrm{cm}^{-1}$ 

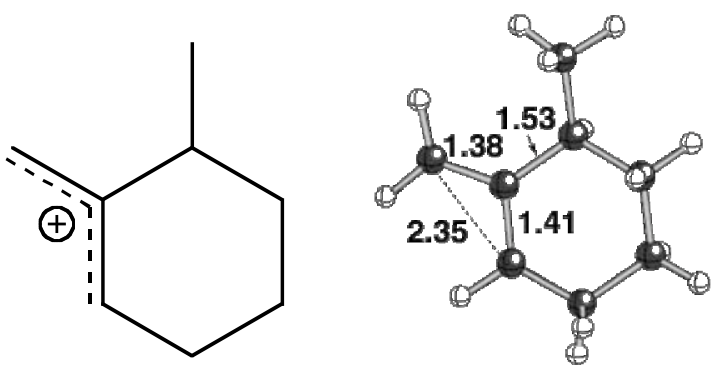

b3lyp/6-31G(d)

Coordinates:

Standard orientation:

\begin{tabular}{|c|c|c|c|c|}
\hline \multirow{2}{*}{$\begin{array}{l}\text { Center } \\
\text { Number }\end{array}$} & \multirow{2}{*}{$\begin{array}{l}\text { Atomic } \\
\text { Number }\end{array}$} & \multirow{2}{*}{$\begin{array}{c}\text { Atomic } \\
\text { Type }\end{array}$} & \multicolumn{2}{|c|}{ Coordinates (Angstron } \\
\hline & & & X $\quad Y$ & $Y \quad Z$ \\
\hline 1 & 6 & 1.581177 & 1.744785 & -0.045091 \\
\hline 2 & 6 & 0.549428 & 0.837283 & -0.105168 \\
\hline 3 & 6 & -0.733556 & 1.391959 & 0.090669 \\
\hline 4 & 1 & -0.809013 & 2.467530 & 0.257005 \\
\hline 5 & 6 & 0.718421 & -0.661937 & -0.344885 \\
\hline 6 & 6 & -1.969843 & 0.614545 & 0.132491 \\
\hline 7 & 1 & -2.748741 & 1.150730 & -0.436404 \\
\hline 8 & 6 & -0.483862 & -1.428295 & 0.246709 \\
\hline 9 & 6 & -1.8 & -0.866382 & -0.242449 \\
\hline 10 & 1 & -1.8 & -0.967374 & $4 \quad-1.332212$ \\
\hline 11 & 1 & -0.399368 & -2.482902 & -0.032170 \\
\hline 12 & 1 & -2.328929 & 0.740044 & 1.178454 \\
\hline 13 & 1 & 0.692338 & -0.799025 & -1.436888 \\
\hline 14 & 1 & -0.436828 & -1.390980 & 1.344450 \\
\hline 15 & 1 & -2.660459 & -1.430403 & 0.179858 \\
\hline 16 & 1 & 2.621015 & 1.448769 & -0.153244 \\
\hline 17 & 1 & 1.394580 & 2.804212 & 0.115414 \\
\hline 18 & 6 & 2.058600 & -1.207116 & 0.170572 \\
\hline 19 & 1 & 2.917054 & -0.775486 & -0.354259 \\
\hline 20 & 1 & 2.179215 & -1.026249 & 1.245090 \\
\hline 21 & 1 & 2.099092 & -2.287921 & 0.007809 \\
\hline
\end{tabular}

Energy: $\mathrm{HF}=-312.3830689$

Zero-Point Energy Correction: 0.190959

Imaginary Frequency: Minimum (No imaginary frequencies) 


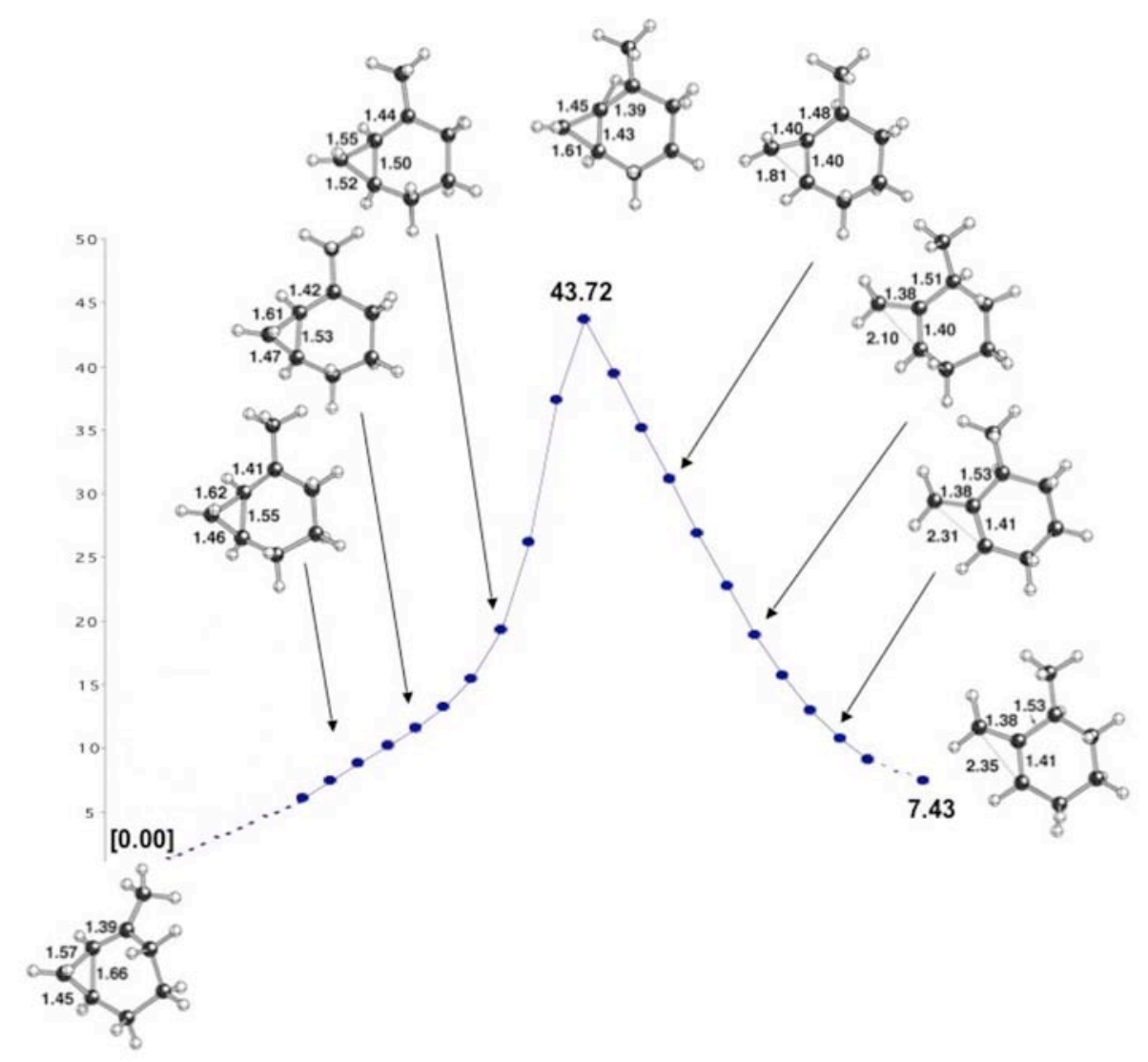



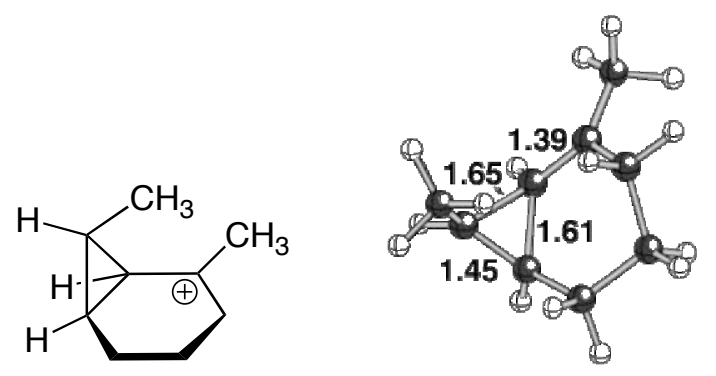

b31yp/6-31G(d)

\section{Coordinates:}

Standard orientation:

\begin{tabular}{|c|c|c|c|c|}
\hline \multirow{2}{*}{$\begin{array}{l}\text { Center } \\
\text { Number }\end{array}$} & \multirow{2}{*}{$\begin{array}{l}\text { Atomic } \\
\text { Number }\end{array}$} & \multirow{2}{*}{$\begin{array}{r}\text { Atomic } \\
\text { Type }\end{array}$} & \multicolumn{2}{|c|}{ Coordinates (Angstron } \\
\hline & & & X $\quad Y$ & $\mathrm{Z}$ \\
\hline 1 & 6 & 1.817979 & -1.127923 & 1.170433 \\
\hline 2 & 1 & 2.914 & -1.093210 & 1.219891 \\
\hline 3 & 1 & 1.502071 & -2.083287 & 1.598475 \\
\hline 4 & 1 & 1.441491 & -0.315225 & 1.792906 \\
\hline 5 & 6 & 1.436 & -1.032753 & -0.282186 \\
\hline 6 & 1 & 37 & -1.863059 & -0.878682 \\
\hline 7 & 6 & -0.129001 & -0.842833 & -0.784159 \\
\hline 8 & 6 & 56 & 0.165 & -1.021453 \\
\hline 9 & 1 & 0 & 0.155 & -2.083446 \\
\hline 10 & 6 & 21 & -0.409892 & 0.009644 \\
\hline 11 & 6 & 1.027241 & 1.540690 & -0.355953 \\
\hline 12 & 1 & 1.383343 & 2.279907 & -1.080376 \\
\hline 13 & 6 & 45 & 0.779604 & 0.903957 \\
\hline 14 & 6 & 0 & 1.937381 & 0.119431 \\
\hline 15 & 1 & -1.008 & 2.202715 & -0.736064 \\
\hline 16 & 1 & -2.035464 & 1.076707 & 1.281649 \\
\hline 17 & 1 & 1.723542 & 1.571280 & 0.489248 \\
\hline 18 & 1 & -0.355658 & -1.550458 & -1.574339 \\
\hline 19 & 1 & -0.439 & 0.527617 & 1.780367 \\
\hline 20 & 1 & $-0.323 t$ & 2.820612 & 0.762738 \\
\hline 21 & 6 & -2.523571 & -1.041433 & -0.122837 \\
\hline 22 & 1 & -2.874054 & -1.355357 & 0.871270 \\
\hline 23 & 1 & -3.251990 & -0.290628 & -0.463048 \\
\hline 24 & 1 & -2.539997 & -1.895681 & -0.801852 \\
\hline
\end{tabular}

Energy: $\mathrm{HF}=-351.7114783$

Zero-Point Energy Correction: $\mathbf{0 . 2 2 0 2 7 7}$

Imaginary Frequency: Minimum (No imaginary frequencies) 

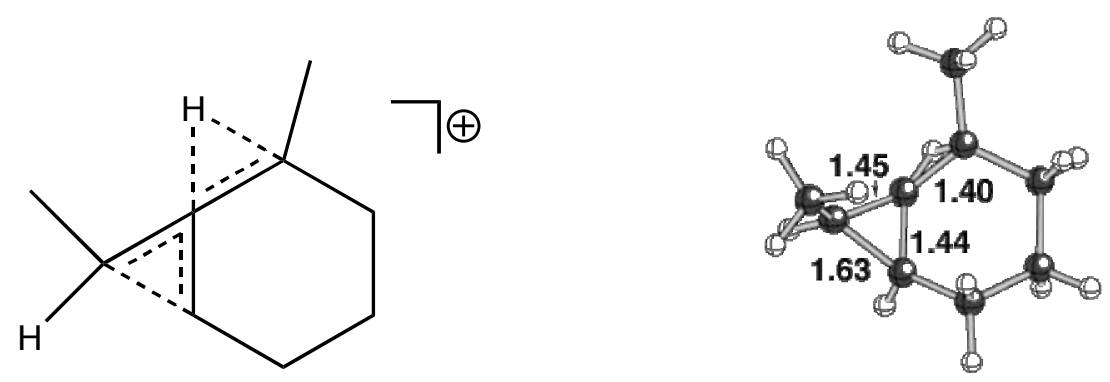

b31yp/6-31G(d)

\section{Coordinates:}

Standard orientation:

\begin{tabular}{|c|c|c|c|c|c|}
\hline \multirow{2}{*}{$\begin{array}{l}\text { Center } \\
\text { Number }\end{array}$} & \multirow{2}{*}{\multicolumn{2}{|c|}{$\begin{array}{l}\text { Atomic } \\
\text { Number }\end{array}$}} & Atomic & \multicolumn{2}{|c|}{ Coordinates (Angstroms } \\
\hline & & & Type & X $\quad Y$ & Z \\
\hline 1 & 6 & 0 & -2.562197 & -0.051202 & 0.737883 \\
\hline 2 & 1 & 0 & -3.232341 & -0.887738 & 0.960778 \\
\hline 3 & 1 & 0 & -3.180040 & 0.838516 & 0.576298 \\
\hline 4 & 1 & 0 & -1.927293 & 0.122092 & 1.611552 \\
\hline 5 & 6 & 0 & -1.757265 & -0.372922 & -0.500771 \\
\hline 6 & 1 & 0 & -2.335380 & -0.589209 & -1.396141 \\
\hline 7 & 6 & 0 & -0.417871 & 0.161257 & -0.683623 \\
\hline 8 & 6 & 0 & -0.407138 & -1.272034 & -0.595907 \\
\hline 9 & 1 & 0 & -0.469621 & -1.868818 & -1.502395 \\
\hline 10 & 6 & 0 & 0.538853 & 1.077038 & -0.246970 \\
\hline 11 & 6 & 0 & 0.567770 & -1.741641 & 0.478643 \\
\hline 12 & 1 & 0 & 0.659111 & -2.830508 & 0.478451 \\
\hline 13 & 6 & 0 & 1.872582 & 0.471478 & 0.181879 \\
\hline 14 & 6 & 0 & 1.929246 & -1.077187 & 0.154564 \\
\hline 15 & 1 & 0 & 2.260076 & -1.418765 & -0.833423 \\
\hline 16 & 1 & 0 & 2.703283 & 0.912446 & -0.380333 \\
\hline 17 & 1 & 0 & 0.218838 & -1.446087 & 1.476041 \\
\hline 18 & 1 & 0 & 0.326432 & 0.927977 & -1.530590 \\
\hline 19 & 1 & 0 & 1.997459 & 0.834479 & 1.212257 \\
\hline 20 & 1 & 0 & 2.694254 & -1.406323 & 0.864355 \\
\hline 21 & 6 & 0 & 0.214211 & 2.507256 & 0.107794 \\
\hline 22 & 1 & 0 & 0.108660 & 2.576163 & 1.197406 \\
\hline 23 & 1 & 0 & 1.026262 & 3.179126 & -0.185239 \\
\hline 24 & 1 & 0 & -0.718846 & 2.844387 & -0.349969 \\
\hline
\end{tabular}

Energy: $\mathrm{HF}=-351.6471497$

Zero-Point Energy Correction: $\mathbf{0 . 2 1 5 6 8 0}$

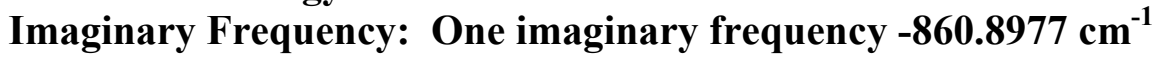



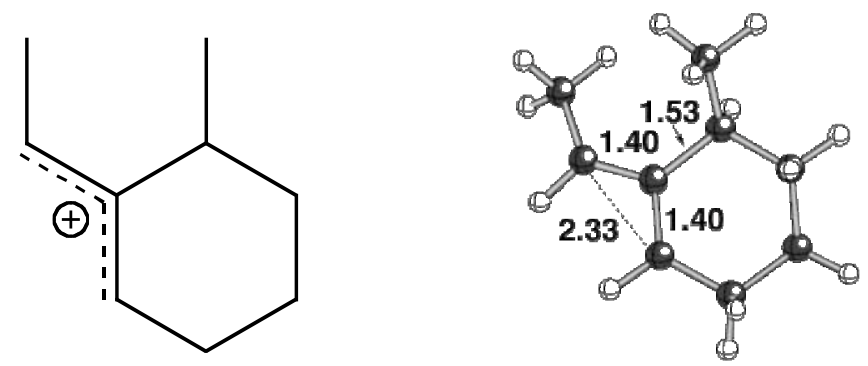

b3lyp/6-31G(d)

\section{Coordinates:}

Standard orientation:

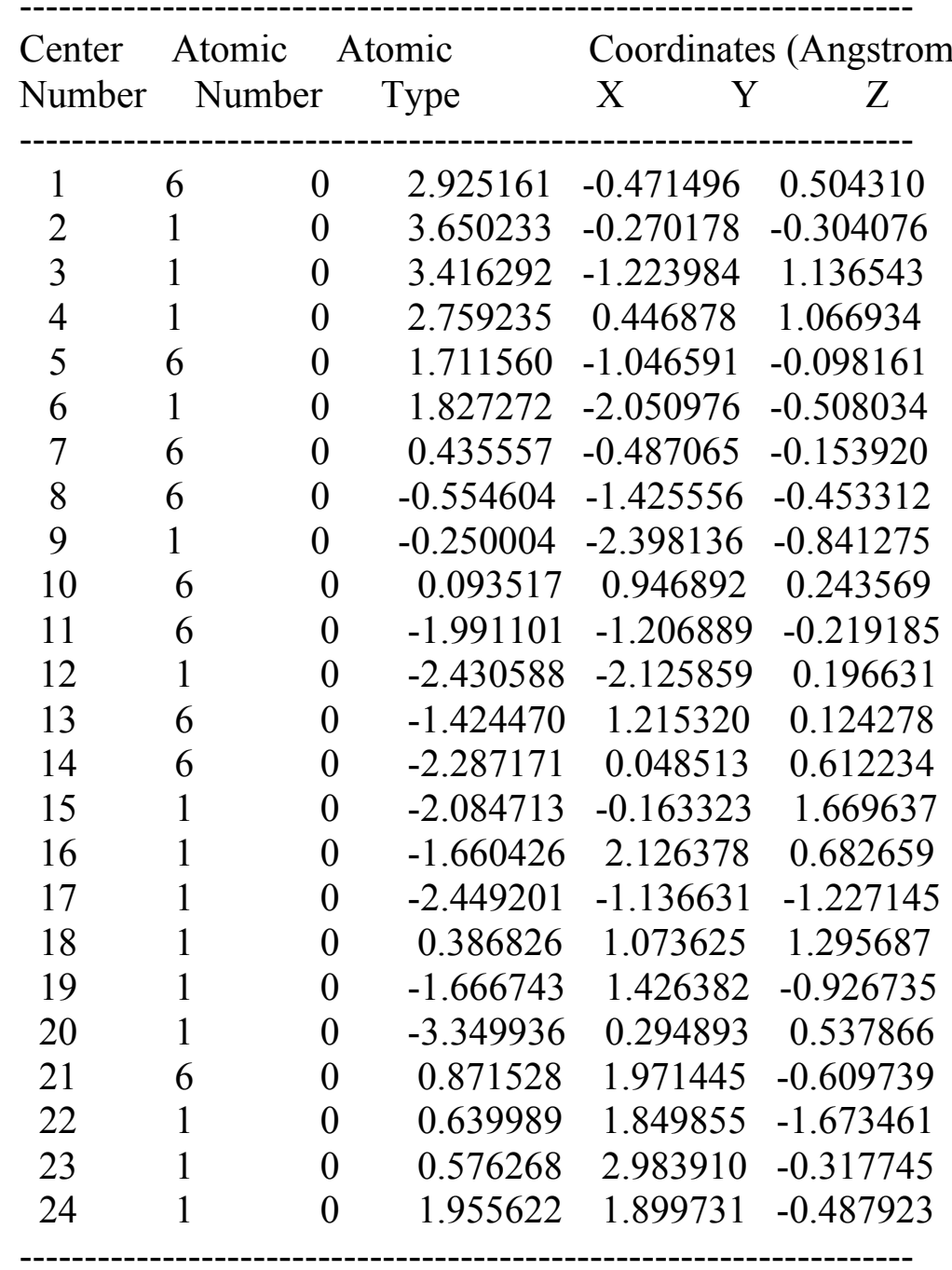

Energy: $\mathrm{HF}=-351.7157794$ 


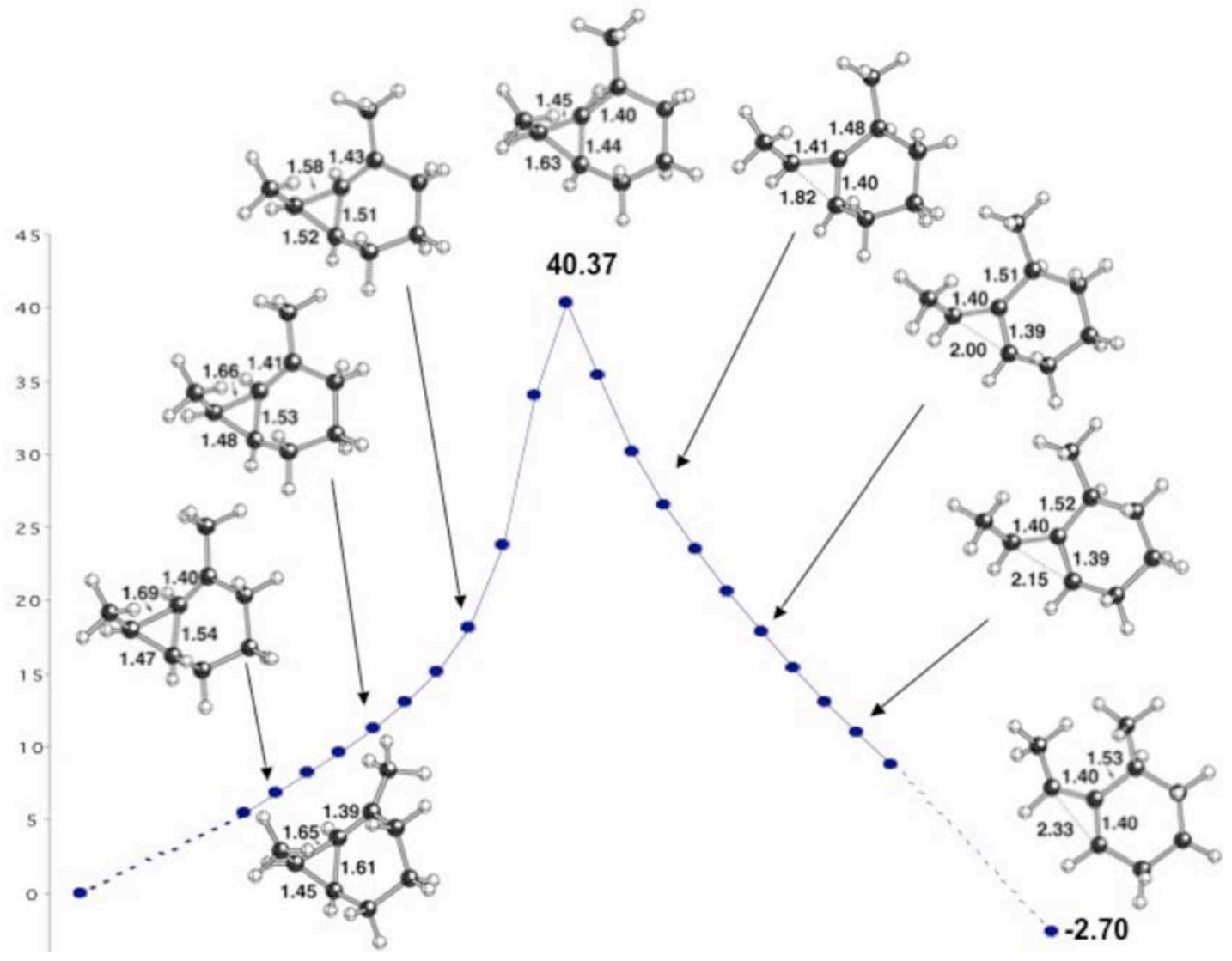



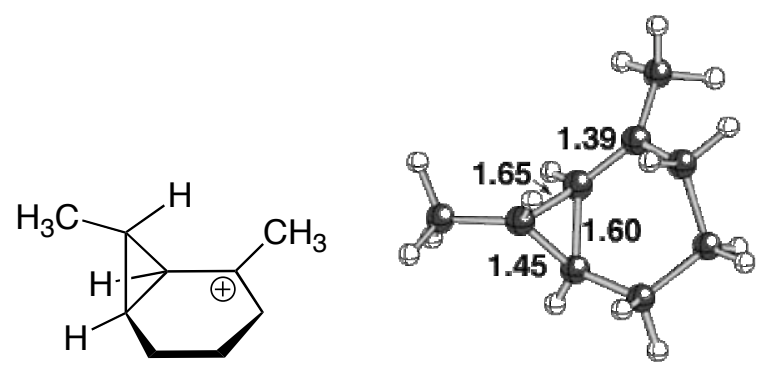

b3lyp/6-31G(d)

\section{Coordinates:}

Standard orientation:

\begin{tabular}{|c|c|c|c|c|}
\hline \multirow{2}{*}{$\begin{array}{l}\text { Center } \\
\text { Number }\end{array}$} & \multirow{2}{*}{$\begin{array}{l}\text { Atomic } \\
\text { Number }\end{array}$} & \multirow{2}{*}{$\begin{array}{r}\text { Atomic } \\
\text { Type }\end{array}$} & \multicolumn{2}{|c|}{ Coordinates (Angstroms) } \\
\hline & & & X $\quad Y$ & I $\quad \mathrm{Z}$ \\
\hline 1 & 6 & 1.530642 & -0.035718 & 0.432609 \\
\hline 2 & 6 & 0.344365 & -0.692954 & -0.500854 \\
\hline 3 & 6 & 0.938582 & 0.788658 & -0.596566 \\
\hline 4 & 1 & 1.506807 & 0.875580 & -1.521626 \\
\hline 5 & 6 & -0.930788 & -0.859890 & 0.027289 \\
\hline 6 & 6 & 0.002224 & 1.951737 & -0.248538 \\
\hline 7 & 1 & 0.063559 & 2.691370 & -1.052523 \\
\hline 8 & 6 & -1.570727 & 0.245193 & 0.801400 \\
\hline 9 & 6 & -1.462964 & 1.550400 & -0.039233 \\
\hline 10 & 1 & -1.960381 & 1.394889 & -1.003364 \\
\hline 11 & 1 & -2.611042 & 0.001388 & 1.032105 \\
\hline 12 & 1 & 0.382125 & 2.436024 & 0.658755 \\
\hline 13 & 1 & 0.728628 & -1.432916 & -1.194599 \\
\hline 14 & 1 & -1.052942 & 0.394256 & 1.760337 \\
\hline 15 & 1 & -2.006650 & 2.348308 & 0.474896 \\
\hline 16 & 6 & 2.869926 & -0.712593 & 0.306206 \\
\hline 17 & 1 & 3.626216 & -0.062844 & 0.764635 \\
\hline 18 & 1 & 2.889513 & -1.669769 & 0.835365 \\
\hline 19 & 1 & 3.152882 & -0.873244 & -0.738252 \\
\hline 20 & 1 & 1.180401 & 0.127043 & 1.450215 \\
\hline 21 & 6 & -1.723658 & -2.080578 & -0.265951 \\
\hline 22 & 1 & -1.188141 & -2.815328 & -0.869607 \\
\hline 23 & 1 & -2.039899 & -2.542485 & 0.680841 \\
\hline 24 & 1 & -2.656688 & -1.797808 & -0.775356 \\
\hline
\end{tabular}

Energy: $\mathrm{HF}=-351.7176934$

Zero-Point Energy Correction: $\mathbf{0 . 2 2 0 0 3 3}$

Imaginary Frequency: Minimum (No imaginary frequencies) 


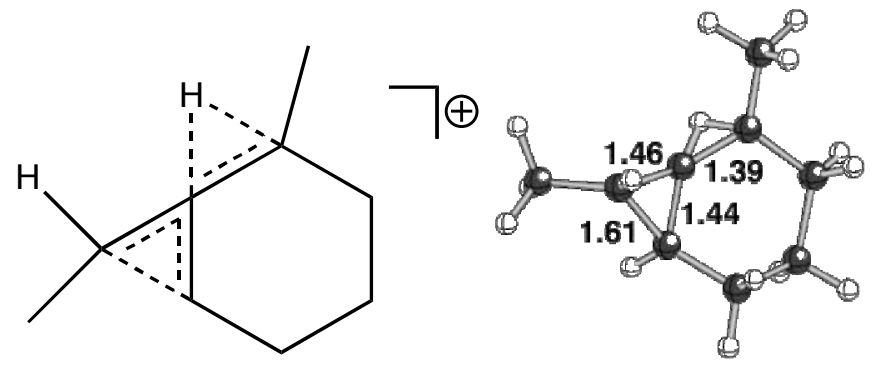

b31yp/6-31G(d)

\section{Coordinates:}

Standard orientation:

\begin{tabular}{|c|c|c|c|c|c|}
\hline Center & Atomic & & Atomic & Coordinate & es (Angst \\
\hline Number & Numbe & & Type & $\begin{array}{ll}X & Y\end{array}$ & $\mathrm{Z}$ \\
\hline 1 & 6 & 0 & -1.714141 & 0.092587 & 0.424473 \\
\hline 2 & 6 & 0 & -0.428588 & 0.339855 & -0.212325 \\
\hline 3 & 6 & 0 & -0.728346 & -1.060488 & -0.118620 \\
\hline 4 & 1 & 0 & -1.225570 & -1.559212 & -0.947973 \\
\hline 5 & 6 & 0 & 0.802239 & 0.991668 & -0.174374 \\
\hline 6 & 6 & 0 & 418561 & -1.809638 & 0.555219 \\
\hline 7 & 1 & 0 & 0.247590 & -2.888815 & 0.539929 \\
\hline 8 & 6 & 0 & 2.021715 & 0.070984 & -0.217587 \\
\hline 9 & 6 & 0 & 35 & -1.445827 & 33434 \\
\hline 10 & 1 & 0 & 585584 & -1.789599 & -1.26832 \\
\hline 11 & 1 & 0 & 2.692368 & 0.345511 & -1.039083 \\
\hline 12 & 1 & 0 & 0.507580 & -1.509428 & 1.606942 \\
\hline 13 & 1 & 0 & 0.160 & 0.982096 & -1.294650 \\
\hline 14 & 1 & 0 & & 0.329248 & 0.700898 \\
\hline 15 & 1 & & 2.564082 & -1.982679 & 0.172285 \\
\hline 16 & 6 & 0 & -3.066691 & 0.178878 & -0.256103 \\
\hline 17 & 1 & 0 & -3.768694 & -0.503765 & 0.230360 \\
\hline 18 & 1 & 0 & & 1.195513 & -0.166184 \\
\hline 19 & 1 & 0 & -3.008243 & -0.076627 & -1.318108 \\
\hline 20 & 1 & 0 & -1.718332 & 0.363601 & 1.482868 \\
\hline 21 & 6 & 0 & 0.962590 & 2.430264 & 0.256982 \\
\hline 22 & 1 & 0 & 0.034936 & 2.998004 & 0.150922 \\
\hline 23 & 1 & 0 & & 2.442084 & 1.313927 \\
\hline 24 & 1 & 0 & 1.757366 & 2.924366 & -0.309198 \\
\hline
\end{tabular}

Energy: $\mathrm{HF}=-351.6457847$

Zero-Point Energy Correction: $\mathbf{0 . 2 1 5 6 8 4}$

Imaginary Frequency: One imaginary frequency $\mathbf{- 8 4 2 . 5 0 6 2} \mathrm{cm}^{-1}$ 

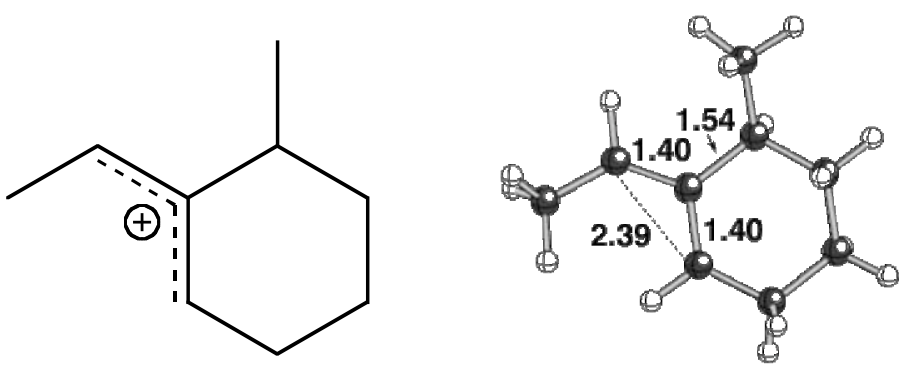

b3lyp/6-31G(d)

Coordinates:

Standard orientation:

\begin{tabular}{|c|c|c|c|c|c|}
\hline Center & Atomic & & Atomic & Coordinate & es (Angsti \\
\hline Number & Numbe & & Type & $X$ & $\mathrm{Z}$ \\
\hline 1 & 6 & 0 & -1.943978 & 0.213548 & -0.15197 \\
\hline 2 & 6 & 0 & -0.572424 & -0.043094 & -0.106756 \\
\hline 3 & 6 & 0 & -0.156751 & -1.354678 & 0.128435 \\
\hline 4 & 1 & 0 & -0.906 & -2.119258 & 0.320059 \\
\hline 5 & 6 & 0 & 0.460279 & 1.069990 & -0.341811 \\
\hline 6 & 6 & 0 & 1.244784 & -1.795722 & 0.160730 \\
\hline 7 & 1 & 0 & 1.328303 & -2.743043 & -0.396622 \\
\hline 8 & 6 & 0 & 1.836929 & 0.651576 & 0.213855 \\
\hline 9 & 6 & 0 & 2.263105 & -0.735864 & -0.274883 \\
\hline 10 & 1 & 0 & 341991 & -0.739169 & -1.368987 \\
\hline 11 & 1 & 0 & 2.577249 & 1.399467 & -0.086280 \\
\hline 12 & 1 & 0 & 23 & -2.118303 & 1.208805 \\
\hline 13 & 1 & 0 & & 1.176972 & -1.432343 \\
\hline 14 & 1 & & 1.805092 & 0.667324 & 1.312866 \\
\hline 15 & 1 & 0 & 3.251959 & -0.998754 & 0.111246 \\
\hline 16 & 6 & 0 & -3.098161 & -0.686151 & 0.033875 \\
\hline 17 & 1 & 0 & -3.741352 & -0.268093 & 0.823776 \\
\hline 18 & 1 & 0 & & -0.6464 & $-0.87460 \&$ \\
\hline 19 & 1 & 0 & -2.871392 & -1.726050 & 0.267045 \\
\hline 20 & 1 & 0 & -2.227782 & 1.243517 & -0.367484 \\
\hline 21 & 6 & 0 & 0.028913 & 2.430825 & 0.230909 \\
\hline 22 & 1 & 0 & -0.848521 & 2.850304 & -0.271945 \\
\hline 23 & 1 & 0 & -0.182823 & 2.366040 & 1.304601 \\
\hline 24 & 1 & 0 & 0.839536 & 3.152957 & 0.095621 \\
\hline
\end{tabular}

Energy: $\mathrm{HF}=-351.7131624$

Zero-Point Energy Correction: 0.219189

Imaginary Frequency: Minimum (No imaginary frequencies) 


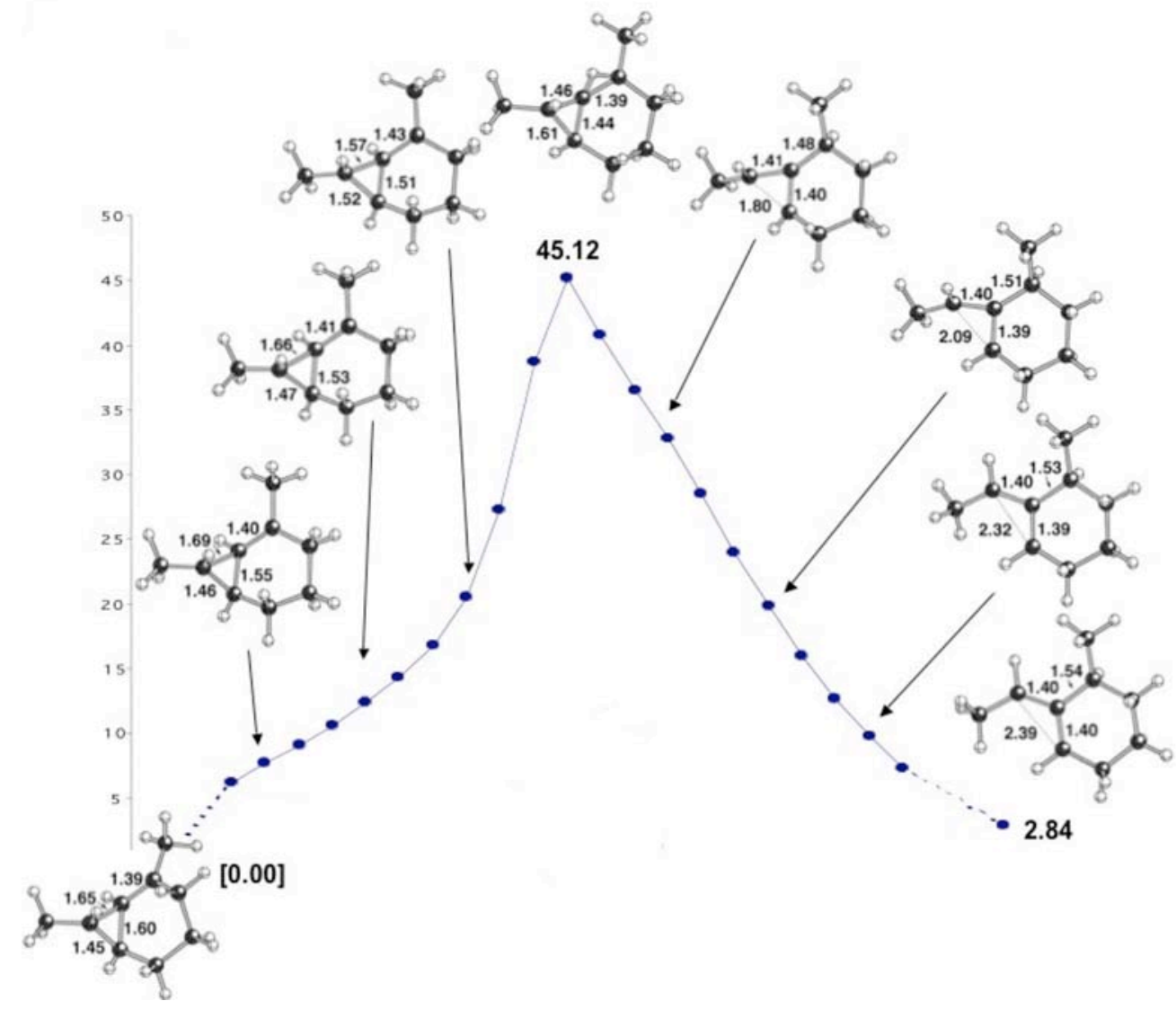



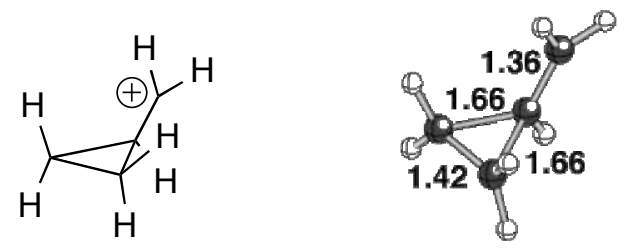

b3lyp/6-31G(d)

Coordinates:

Standard orientation:

\begin{tabular}{|c|c|c|c|c|}
\hline \multirow{2}{*}{$\begin{array}{l}\text { Center } \\
\text { Number }\end{array}$} & \multirow{2}{*}{$\begin{array}{l}\text { Atomic } \\
\text { Number }\end{array}$} & \multirow{2}{*}{$\begin{array}{r}\text { Atomic } \\
\text { Type }\end{array}$} & \multicolumn{2}{|c|}{ Coordinates (Angstro1 } \\
\hline & & & $\begin{array}{ll}X & Y\end{array}$ & $Y \quad Z$ \\
\hline 1 & 6 & -0.958774 & -0.704616 & -0.153428 \\
\hline 2 & 6 & & -0.017495 & 0.5 \\
\hline 3 & 6 & -0.95 & 0.714761 & -0.131434 \\
\hline 4 & 6 & 1.525754 & 0.003908 & -0.207643 \\
\hline 5 & 1 & 22 & -0.034366 & 1.612235 \\
\hline 6 & 1 & -0.748 & -1.242807 & -1.072282 \\
\hline 7 & & & 1.28 & -1.030948 \\
\hline 8 & ( & 69 & 0.003197 & 0.269824 \\
\hline 9 & 1 & -1.447578 & 1.258610 & 0.671094 \\
\hline 10 & 1 & -1.462649 & -1.268306 & $\begin{array}{ll}6 & 0.628105\end{array}$ \\
\hline 11 & 1 & 1.510636 & 0.022711 & -1.296095 \\
\hline
\end{tabular}

Energy: $H F=-156.2964504$

Zero-Point Energy Correction: 0.097667

Imaginary Frequency: Minimum (No imaginary frequencies) 


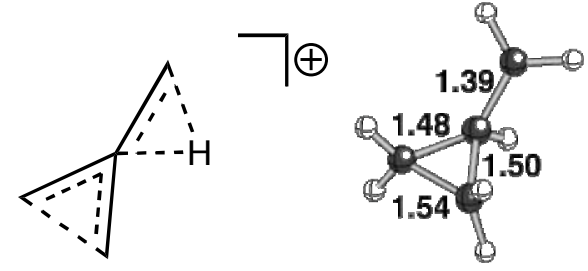

b3lyp/6-31G(d)

\section{Coordinates:}

Standard orientation:

\begin{tabular}{|c|c|c|c|c|}
\hline \multirow{2}{*}{$\begin{array}{l}\text { Center } \\
\text { Number }\end{array}$} & \multirow{2}{*}{$\begin{array}{l}\text { Atomic } \\
\text { Number }\end{array}$} & \multirow{2}{*}{$\begin{array}{r}\text { Atomic } \\
\text { Type }\end{array}$} & \multicolumn{2}{|c|}{ Coordinates (Angstrom } \\
\hline & & & $X \quad Y$ & Z Z \\
\hline 1 & 6 & 0.942876 & 0.780027 & -0.047988 \\
\hline 2 & 6 & -0.279125 & -0.010075 & 0.201387 \\
\hline 3 & 6 & 0.990656 & -0.754512 & -0.100156 \\
\hline 4 & 6 & -1.630910 & -0.000022 & -0.120077 \\
\hline 5 & 1 & -0.720034 & -0.163846 & 1.274298 \\
\hline 6 & 1 & 0.990848 & 1.345263 & -0.975324 \\
\hline 7 & 1 & 1.031886 & -1.266996 & -1.057881 \\
\hline 8 & 1 & -2.209107 & -0.927047 & -0.109930 \\
\hline 9 & 1 & 1.500610 & -1.257572 & 0.715678 \\
\hline 10 & 1 & 1.445277 & 1.240819 & 0.798775 \\
\hline 11 & 1 & -2.180461 & 0.936868 & -0.244611 \\
\hline
\end{tabular}

Energy: $H F=-156.2446117$

Zero-Point Energy Correction: 0.093583

Imaginary Frequency: One imaginary frequency $-660.9954 \mathrm{~cm}^{-1}$ 

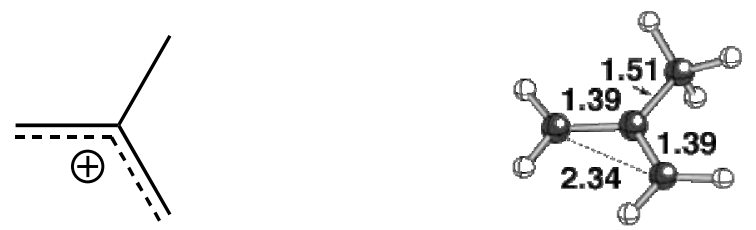

b3lyp/6-31G(d)

Coordinates:

Standard orientation:

\begin{tabular}{|c|c|c|c|c|c|}
\hline \multirow{2}{*}{$\begin{array}{l}\text { Center } \\
\text { Number }\end{array}$} & \multirow{2}{*}{$\begin{array}{l}\text { Atomic } \\
\text { Number }\end{array}$} & \multirow{2}{*}{\multicolumn{2}{|c|}{$\begin{array}{l}\text { Atomic } \\
\text { Type }\end{array}$}} & \multicolumn{2}{|c|}{ Coordinates (Angstroms } \\
\hline & & & & X $\quad Y$ & $\mathrm{Z}$ \\
\hline 1 & 6 & 0 & 0.801098 & 1.168523 & -0.001367 \\
\hline 2 & 6 & 0 & 0.046008 & -0.000013 & 0.002767 \\
\hline 3 & 6 & 0 & 0.801305 & -1.168411 & -0.001830 \\
\hline 4 & 6 & 0 & -1.463033 & -0.000093 & 0.000525 \\
\hline 5 & 1 & 0 & -1.838838 & -0.000021 & 1.030504 \\
\hline 6 & 1 & 0 & 0.332685 & 2.151290 & -0.035320 \\
\hline 7 & 1 & 0 & 0.333040 & -2.151298 & -0.034273 \\
\hline 8 & 1 & 0 & -1.858437 & -0.887104 & -0.500737 \\
\hline 9 & 1 & 0 & 1.888975 & -1.145115 & 0.020506 \\
\hline 10 & 1 & 0 & 1.888797 & 1.145400 & 0.019637 \\
\hline 11 & 1 & 0 & -1.858487 & 0.886813 & -0.500886 \\
\hline
\end{tabular}

Energy: $\mathrm{HF}=-156.2976352$

Zero-Point Energy Correction: 0.096524

Imaginary Frequency: Minimum (No imaginary frequencies) 


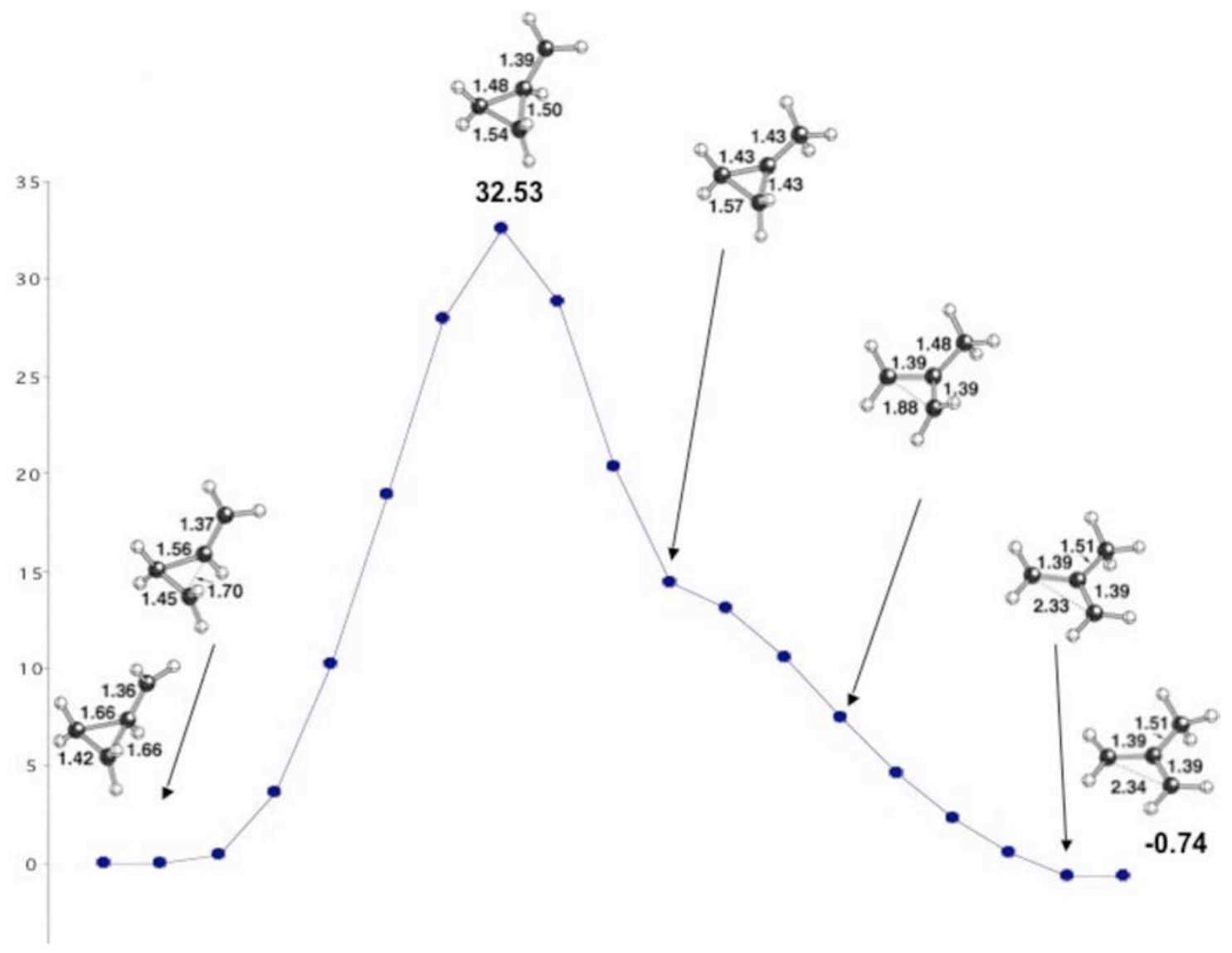




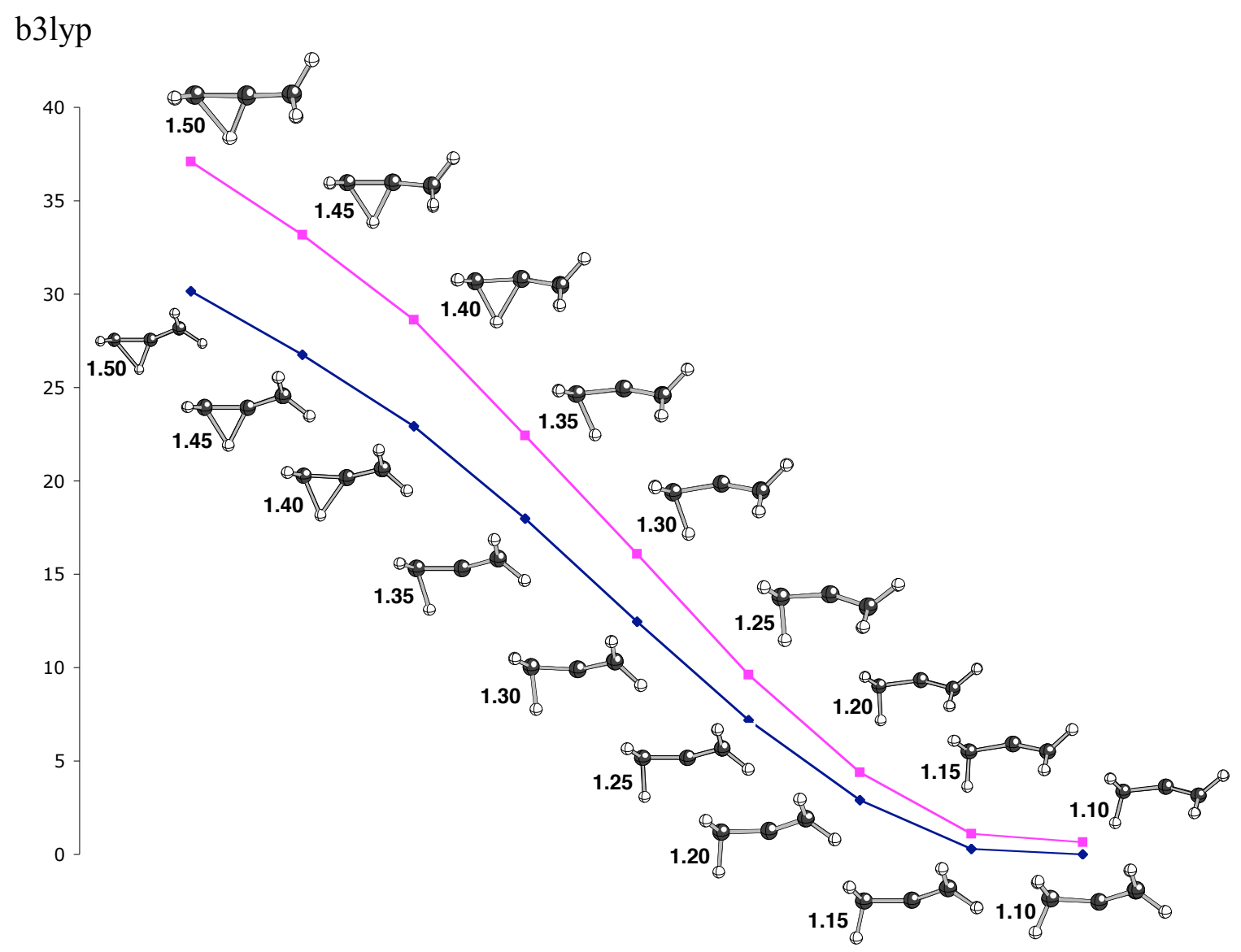


<smiles>C1=CCC=C1</smiles>

MP2/6-31G(d)

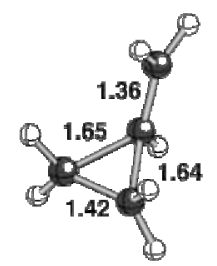

\section{Coordinates:}

Standard orientation:

\begin{tabular}{|c|c|c|c|c|}
\hline \multirow{2}{*}{$\begin{array}{l}\text { Center } \\
\text { Number }\end{array}$} & \multirow{2}{*}{$\begin{array}{l}\text { Atomic } \\
\text { Number }\end{array}$} & \multirow{2}{*}{$\begin{array}{l}\text { Atomic } \\
\text { Type }\end{array}$} & \multicolumn{2}{|c|}{ Coordinates (Angstroms) } \\
\hline & & & X $\quad \mathrm{Y}$ & $\mathrm{Z}$ \\
\hline 1 & 6 & 0.943293 & 0.716986 & -0.125369 \\
\hline 2 & 6 & -0.376046 & -0.031082 & 0.535704 \\
\hline 3 & 6 & 0.949834 & -0.698835 & -0.165191 \\
\hline 4 & 6 & -1.511683 & 0.007055 & -0.206771 \\
\hline 5 & 1 & -0.393750 & -0.062187 & 1.618443 \\
\hline 6 & 1 & 0.706094 & 1.293170 & -1.013164 \\
\hline 7 & 1 & 0.725584 & -1.223238 & -1.087800 \\
\hline 8 & 1 & -2.492558 & 0.004829 & 0.263432 \\
\hline 9 & 1 & 1.466106 & -1.268402 & 0.602522 \\
\hline 10 & 1 & 1.439610 & 1.250553 & 0.680472 \\
\hline 11 & 1 & -1.483476 & 0.040535 & -1.294145 \\
\hline
\end{tabular}

Energy: MP2 $=-155.7257483$

Zero-Point Energy Correction: 0.099750

Imaginary Frequency: Minimum (No imaginary frequencies) 

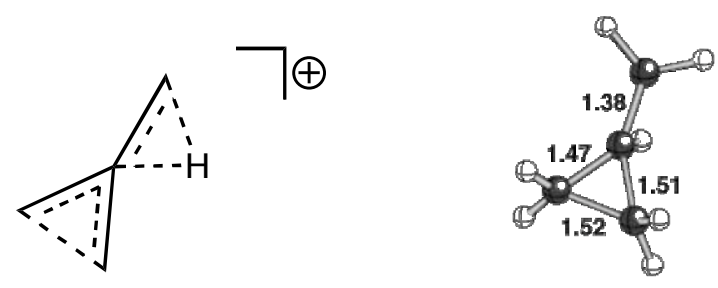

$\mathrm{MP} 2 / 6-31 \mathrm{G}(\mathrm{d})$

\section{Coordinates:}

Standard orientation:

\begin{tabular}{|c|c|c|c|c|}
\hline Center & Atomic & Atomic & Coordinate & tes (Angstron \\
\hline Number & Numbe & r Type & $\begin{array}{ll}X & Y\end{array}$ & $\mathrm{Z}$ \\
\hline 1 & 6 & 0.931400 & 0.779365 & -0.034850 \\
\hline 2 & 6 & -0.282238 & -0.019905 & 0.206876 \\
\hline 3 & 6 & 1.001551 & -0.740529 & -0.113521 \\
\hline 4 & 6 & -1.625890 & 0.000586 & -0.121155 \\
\hline 5 & 1 & -0.706145 & -0.238560 & 1.267705 \\
\hline 6 & 1 & 0.969115 & 1.350800 & -0.957434 \\
\hline 7 & 1 & 1.035908 & -1.226721 & -1.083710 \\
\hline 8 & 1 & -2.214924 & -0.917020 & -0.079384 \\
\hline 9 & 1 & 1.507995 & -1.261667 & 0.691406 \\
\hline 10 & 1 & 1.421887 & 1.237967 & 0.818672 \\
\hline 11 & 1 & -2.162773 & 0.938097 & $7 \quad-0.281355$ \\
\hline
\end{tabular}

Energy: MP2 $=-155.6681411$

Zero-Point Energy Correction: 0.096097

Imaginary Frequency: One imaginary frequency $-782.7792 \mathrm{~cm}^{-1}$ 


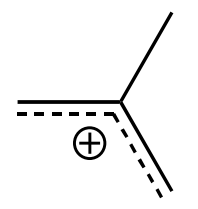

MP2/6-31G(d)

\section{Coordinates:}

Standard orientation:

\begin{tabular}{|c|c|c|c|c|}
\hline \multirow{2}{*}{$\begin{array}{l}\text { Center } \\
\text { Number }\end{array}$} & \multirow{2}{*}{$\begin{array}{l}\text { Atomic } \\
\text { Number }\end{array}$} & \multirow{2}{*}{$\begin{array}{r}\text { Atomic } \\
\text { Type }\end{array}$} & \multicolumn{2}{|c|}{ Coordinates (Angstroms) } \\
\hline & & & X $\quad Y$ & $Y \quad Z$ \\
\hline 1 & 6 & 0.800768 & 1.164644 & -0.000342 \\
\hline 2 & 6 & 0.041499 & -0.000044 & -0.005108 \\
\hline 3 & 6 & 0.801320 & -1.164365 & 0.000067 \\
\hline 4 & 6 & -1.463066 & -0.000256 & 0.001384 \\
\hline 5 & 1 & -1.829631 & 0.001891 & 1.031692 \\
\hline 6 & 1 & 0.338259 & 2.149902 & -0.015368 \\
\hline 7 & 1 & 0.339291 & -2.149821 & -0.016292 \\
\hline 8 & 1 & -1.853936 & -0.887146 & -0.499961 \\
\hline 9 & 1 & 1.888783 & -1.130462 & 0.013439 \\
\hline 10 & 1 & 1.888238 & 1.131344 & 0.014214 \\
\hline 11 & 1 & -1.854134 & 0.884416 & $5-0.503725$ \\
\hline
\end{tabular}

Energy: MP2 $=-155.7204362$

Zero-Point Energy Correction: 0.098297

Imaginary Frequency: Minimum (No imaginary frequencies) 


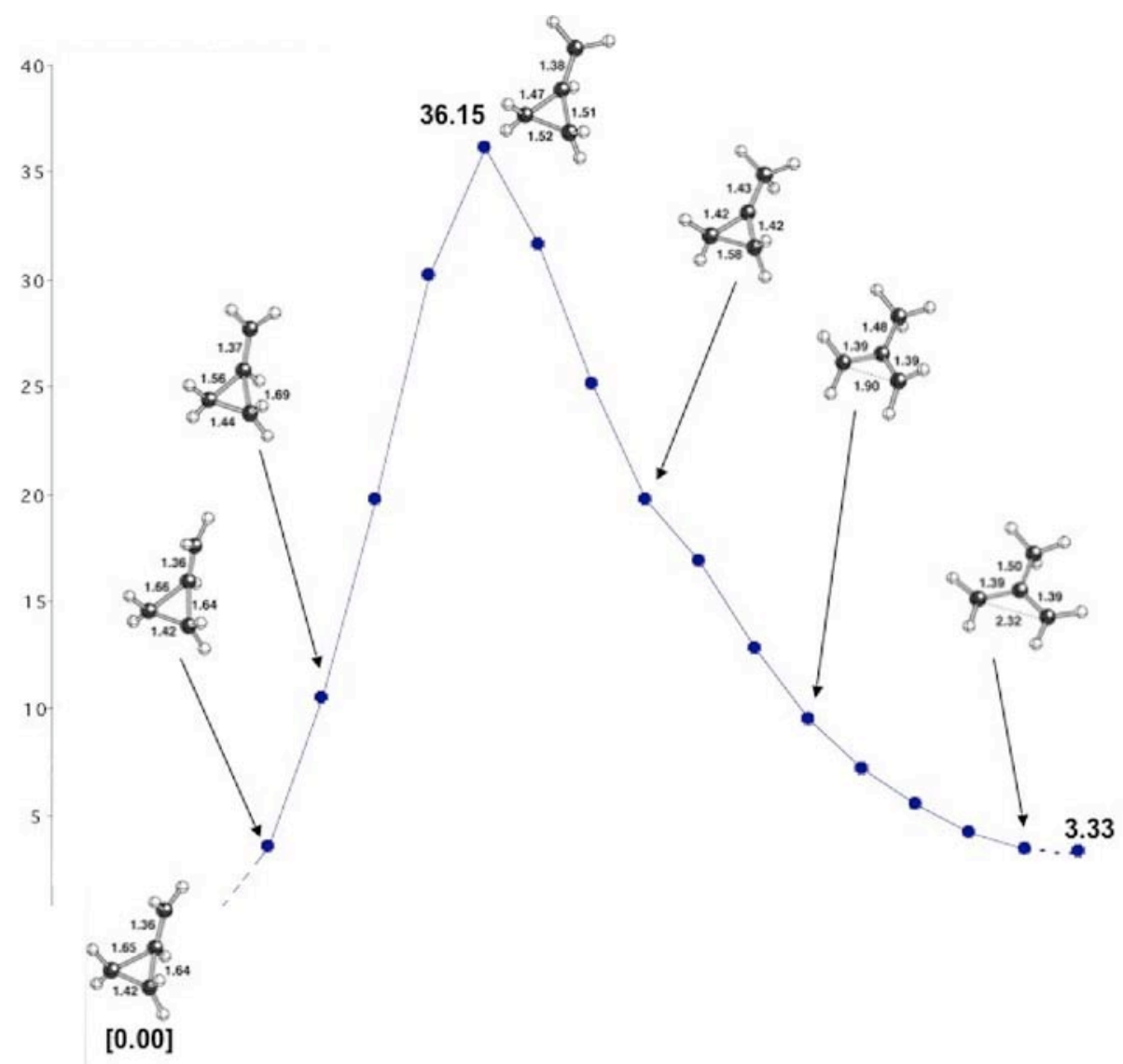




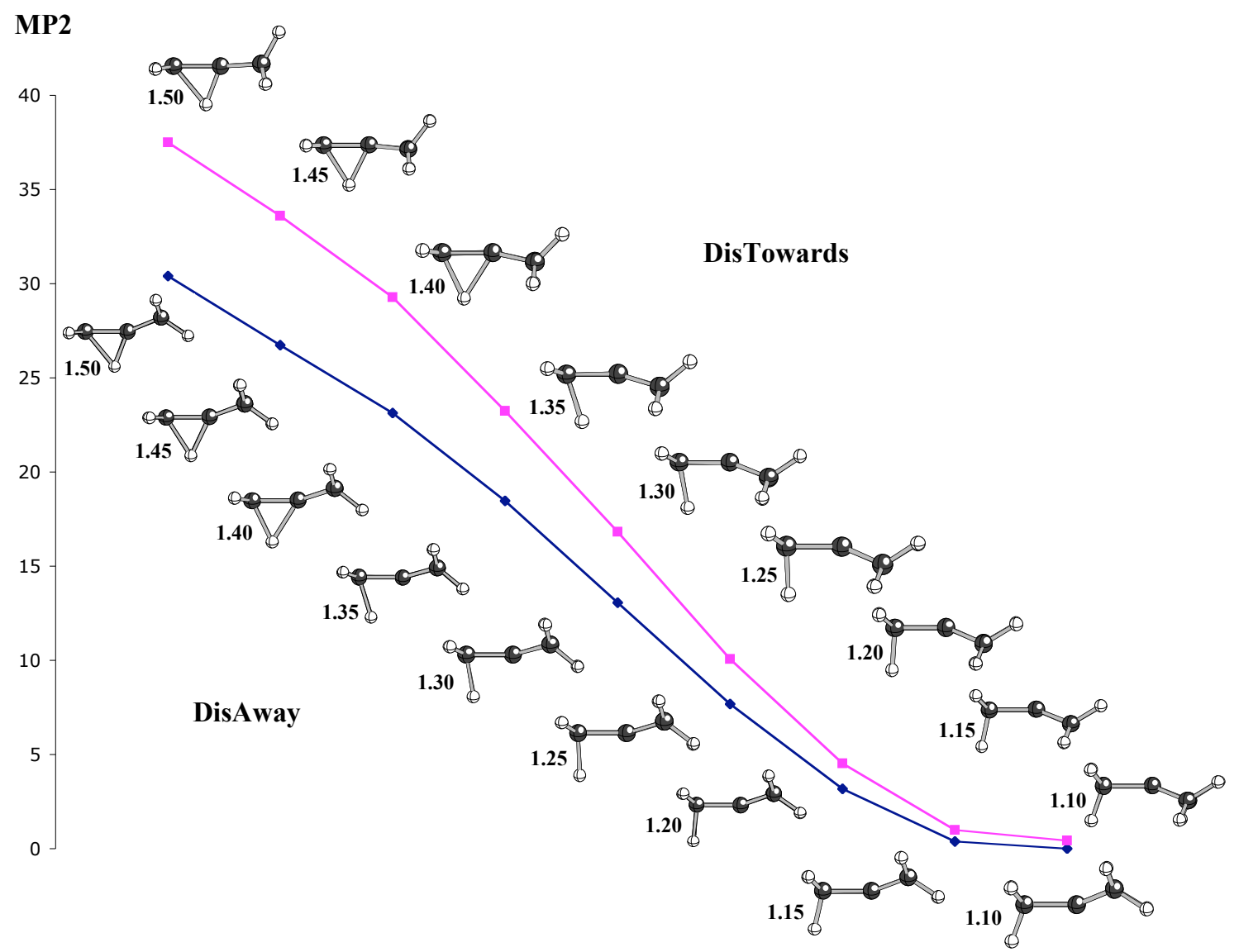




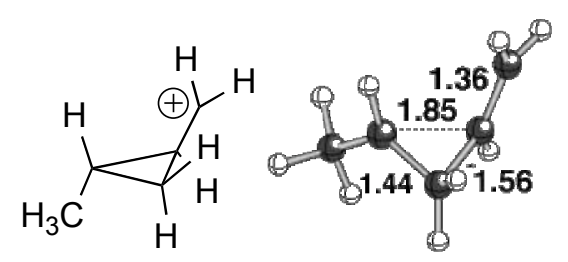

b31yp/6-31G(d)

\section{Coordinates:}

Standard orientation:

\begin{tabular}{|c|c|c|c|c|}
\hline \multirow{2}{*}{$\begin{array}{l}\text { Center } \\
\text { Number }\end{array}$} & \multirow{2}{*}{$\begin{array}{l}\text { Atomic } \\
\text { Number }\end{array}$} & \multirow{2}{*}{$\begin{array}{r}\text { Atomic } \\
\text { Type }\end{array}$} & \multicolumn{2}{|c|}{ Coordinates (Angstroms) } \\
\hline & & & $\begin{array}{ll}\mathrm{X} & \mathrm{Y}\end{array}$ & $\mathrm{Z}$ \\
\hline 1 & 6 & -0.651477 & 0.090697 & -0.520899 \\
\hline 2 & 6 & 0.852074 & -0.004295 & 0.556349 \\
\hline 3 & 6 & 0.053502 & 1.222982 & 0.027860 \\
\hline 4 & 6 & 1.833177 & -0.603761 & -0.162027 \\
\hline 5 & 1 & 2.436888 & -1.398620 & 0.269711 \\
\hline 6 & 1 & 0.609253 & -0.341145 & 1.558843 \\
\hline 7 & 6 & -1.827514 & -0.568638 & 0.102240 \\
\hline 8 & 1 & -2.701496 & -0.221292 & -0.474876 \\
\hline 9 & 1 & -1.800529 & -1.657367 & -0.002350 \\
\hline 10 & 1 & -1.981177 & -0.285251 & 1.145987 \\
\hline 11 & 1 & -0.390514 & -0.221521 & -1.529753 \\
\hline 12 & 1 & 0.618922 & 1.851916 & -0.656009 \\
\hline 13 & 1 & 2.068970 & -0.307968 & -1.181970 \\
\hline 14 & 1 & -0.418885 & 1.759334 & 0.849276 \\
\hline
\end{tabular}

Energy: $\mathrm{HF}=-195.6267033$

Zero-Point Energy Correction: 0.126113

Imaginary Frequency: Minimum (No imaginary frequencies) 

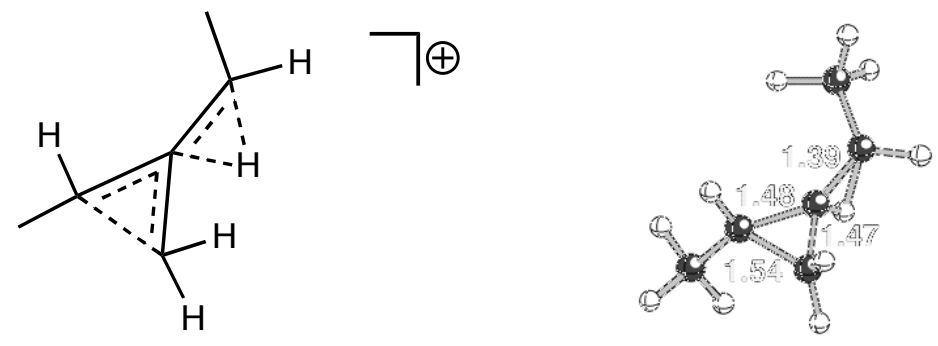

b31yp/6-31G(d)

\section{Coordinates:}

Standard orientation:

\begin{tabular}{|c|c|c|c|c|}
\hline \multirow{2}{*}{$\begin{array}{l}\text { Center } \\
\text { Number }\end{array}$} & \multirow{2}{*}{$\begin{array}{l}\text { Atomic } \\
\text { Number }\end{array}$} & \multirow{2}{*}{$\begin{array}{r}\text { Atomic } \\
\text { Type }\end{array}$} & \multicolumn{2}{|c|}{ Coordinates (Angstroms) } \\
\hline & & & X $\quad Y$ & $\mathrm{Z} \quad \mathrm{Z}$ \\
\hline 1 & 6 & 0.650735 & 0.025010 & 0.500985 \\
\hline 2 & 6 & -0.722626 & -0.029291 & -0.122095 \\
\hline 3 & 6 & -0.032757 & 1.273699 & -0.087122 \\
\hline 4 & 6 & -1.990126 & -0.569902 & 0.027191 \\
\hline 5 & 1 & -2.116725 & -1.624280 & 0.283051 \\
\hline 6 & 1 & -0.834814 & -0.666679 & -1.109349 \\
\hline 7 & 6 & 1.873433 & -0.563117 & -0.168276 \\
\hline 8 & 1 & 2.768828 & -0.060931 & 0.210469 \\
\hline 9 & 1 & 1.966265 & -1.630264 & 0.057753 \\
\hline 10 & 1 & 1.845931 & -0.436796 & -1.255722 \\
\hline 11 & 1 & 0.617659 & -0.082471 & 1.585114 \\
\hline 12 & 1 & -0.436142 & 2.037921 & 0.573122 \\
\hline 13 & 1 & -2.886814 & -0.003520 & -0.238097 \\
\hline 14 & 1 & 0.403863 & 1.648626 & -1.010444 \\
\hline
\end{tabular}

Energy: $\mathrm{HF}=-195.5664105$

Zero-Point Energy Correction: 0.121779

Imaginary Frequency: One imaginary frequency $-736.0972 \mathrm{~cm}^{-1}$ 

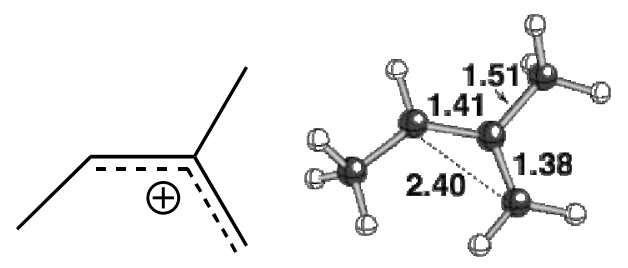

b3lyp/6-31G(d)

\section{Coordinates:}

Standard orientation:

\begin{tabular}{|c|c|c|c|c|}
\hline \multirow{2}{*}{$\begin{array}{l}\text { Center } \\
\text { Number }\end{array}$} & \multirow{2}{*}{$\begin{array}{l}\text { Atomic } \\
\text { Number }\end{array}$} & \multirow{2}{*}{$\begin{array}{r}\text { Atomic } \\
\quad \text { Type }\end{array}$} & \multicolumn{2}{|c|}{ Coordinates (Angstroms) } \\
\hline & & & X $\quad Y$ & I $\quad \mathrm{Z}$ \\
\hline 1 & 6 & 0.695010 & -0.624351 & 0.000015 \\
\hline 2 & 6 & -0.530329 & 0.082481 & -0.000001 \\
\hline 3 & 6 & -0.496024 & 1.457490 & 0.000005 \\
\hline 4 & 6 & -1.829278 & -0.694355 & -0.000007 \\
\hline 5 & 1 & -1.898644 & -1.336420 & 0.884756 \\
\hline 6 & 1 & -1.898593 & -1.336468 & -0.884741 \\
\hline 7 & 6 & 2.072742 & -0.130906 & -0.000010 \\
\hline 8 & 1 & 2.596081 & -0.572074 & 0.866084 \\
\hline 9 & 1 & 2.596064 & -0.572121 & -0.866091 \\
\hline 10 & 1 & 2.203838 & 0.950235 & -0.000040 \\
\hline 11 & 1 & 0.606261 & -1.713692 & 0.000058 \\
\hline 12 & 1 & -1.415168 & 2.038824 & 0.000025 \\
\hline 13 & 1 & -2.692013 & -0.024892 & -0.000057 \\
\hline 14 & 1 & 0.429451 & 2.024450 & -0.000006 \\
\hline
\end{tabular}

Energy: $H F=-195.6350036$

Zero-Point Energy Correction: 0.124638

Imaginary Frequency: Minimum (No imaginary frequencies) 


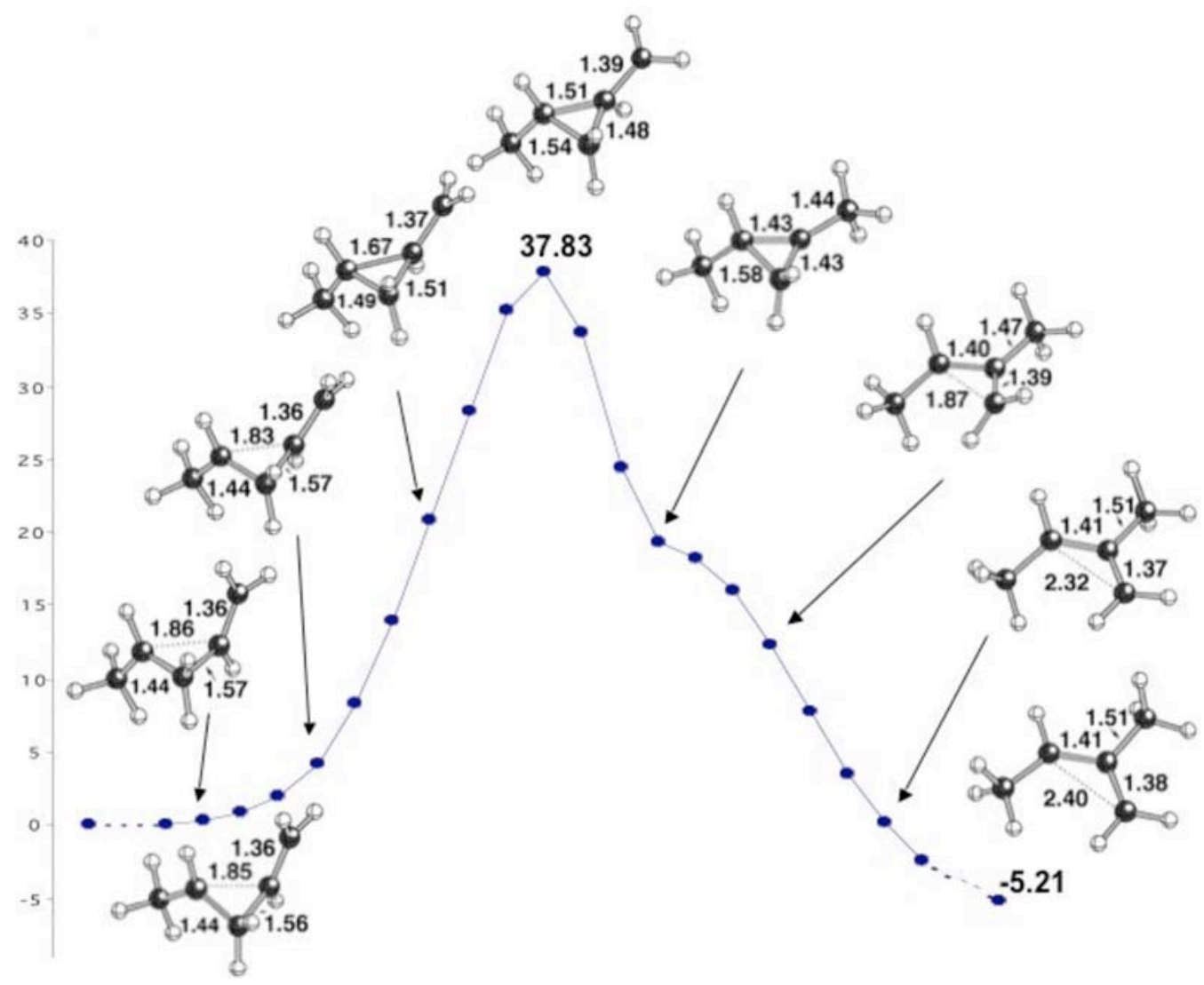


<smiles>CC1CC=CC1</smiles>

b3lyp/6-31G(d)

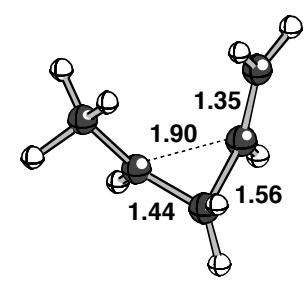

\section{Coordinates:}

Standard orientation:

\begin{tabular}{|c|c|c|c|c|c|}
\hline \multirow{2}{*}{$\begin{array}{l}\text { Center } \\
\text { Number }\end{array}$} & \multirow{2}{*}{\multicolumn{2}{|c|}{$\begin{array}{l}\text { Atomic } \\
\text { Number }\end{array}$}} & \multirow{2}{*}{$\begin{array}{l}\text { Atomic } \\
\text { Type }\end{array}$} & \multicolumn{2}{|c|}{ Coordinates (Angstror } \\
\hline & & & & X $\quad Y$ & $Y \quad Z$ \\
\hline 1 & 6 & 0 & 0.771600 & 0.400488 & -0.391349 \\
\hline 2 & 6 & 0 & -1.120521 & 0.270687 & -0.291767 \\
\hline 3 & 6 & 0 & -0.090478 & 1.165514 & 0.468270 \\
\hline 4 & 6 & 0 & -1.491516 & -0.957785 & 0.132918 \\
\hline 5 & 1 & 0 & -2.311904 & -1.486637 & -0.346397 \\
\hline 6 & 1 & 0 & -1.589716 & 0.726002 & -1.157866 \\
\hline 7 & 1 & 0 & -0.033291 & 0.964998 & 1.536500 \\
\hline 8 & 1 & 0 & -1.008684 & -1.458947 & 0.968124 \\
\hline 9 & 1 & 0 & -0.261788 & 2.212238 & 0.219950 \\
\hline 10 & 6 & 0 & 1.674746 & -0.692404 & 0.043234 \\
\hline 11 & 1 & 0 & 1.733277 & -1.510636 & -0.679591 \\
\hline 12 & 1 & 0 & 2.676620 & -0.228814 & 0.070357 \\
\hline 13 & 1 & 0 & 1.463433 & -1.067594 & 1.046686 \\
\hline 14 & 1 & 0 & 0.869063 & 0.730391 & -1.425598 \\
\hline
\end{tabular}

Energy: $H F=-195.6237445$

Zero-Point Energy Correction: 0.125869

Imaginary Frequency: Minimum (No imaginary frequencies) 
<smiles>CC1C=CC=C1</smiles><smiles>CCCCCCCC</smiles>

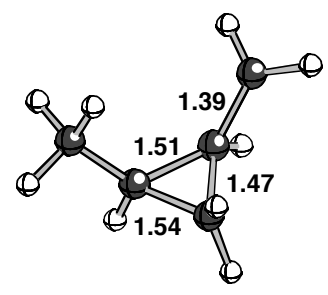

b3lyp/6-31G(d)

\section{Coordinates:}

Standard orientation:

\begin{tabular}{|c|c|c|c|c|}
\hline \multirow{2}{*}{$\begin{array}{l}\text { Center } \\
\text { Number }\end{array}$} & \multirow{2}{*}{$\begin{array}{l}\text { Atomic } \\
\text { Number }\end{array}$} & \multirow{2}{*}{$\begin{array}{l}\text { Atomic } \\
\text { Type }\end{array}$} & \multicolumn{2}{|c|}{ Coordinates (Angstroms } \\
\hline & & & $\mathrm{X} \quad \mathrm{Y}$ & $\mathrm{Z}$ \\
\hline 1 & 6 & -0.733943 & 0.227858 & 0.463081 \\
\hline 2 & 6 & 0.750524 & 0.116533 & 0.219883 \\
\hline 3 & 6 & 0.057003 & 1.306235 & -0.305048 \\
\hline 4 & 6 & 1.751091 & -0.770628 & -0.145207 \\
\hline 5 & 1 & 1.801904 & -1.765263 & 0.302884 \\
\hline 6 & 1 & 1.448768 & 0.134080 & 1.169643 \\
\hline 7 & 1 & -0.050134 & 1.375008 & -1.385825 \\
\hline 8 & 1 & 2.586030 & -0.458385 & -0.777455 \\
\hline 9 & 1 & 0.183700 & 2.257417 & 0.206055 \\
\hline 10 & 6 & -1.682035 & -0.738817 & -0.208624 \\
\hline 11 & 1 & -1.790683 & -1.660403 & 0.372053 \\
\hline 12 & 1 & -2.670580 & -0.272172 & -0.279400 \\
\hline 13 & 1 & -1.349673 & -0.998615 & -1.219140 \\
\hline 14 & 1 & -1.015172 & 0.541251 & 1.466671 \\
\hline
\end{tabular}

Energy: $H F=-195.5672071$

Zero-Point Energy Correction: 0.121789

Imaginary Frequency: One imaginary frequency $-748.6080 \mathrm{~cm}^{-1}$ 

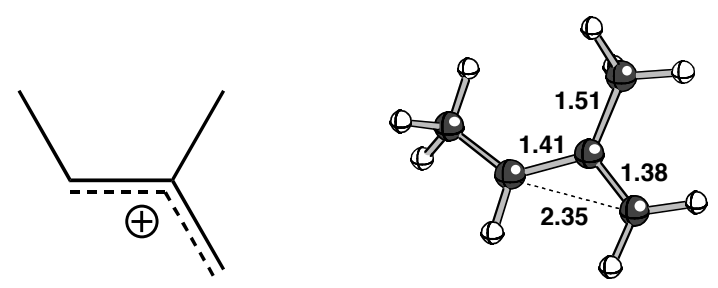

b3lyp/6-31G(d)

\section{Coordinates:}

Standard orientation:

\begin{tabular}{llllcc} 
Center & \multicolumn{2}{c}{ Atomic } & Atomic & \multicolumn{3}{c}{ Coordinates (Angstroms) } \\
Number & Number & Type & X & $Y$ & $Z$ \\
- \hdashline 1 & 6 & 0 & -0.714234 & -0.666552 & -0.000228 \\
2 & 6 & 0 & 0.564040 & -0.069219 & -0.000206 \\
3 & 6 & 0 & 1.612233 & -0.962771 & 0.000070 \\
4 & 6 & 0 & 0.761781 & 1.426915 & -0.000042 \\
5 & 1 & 0 & 0.308115 & 1.883033 & 0.886445 \\
6 & 1 & 0 & 0.304012 & 1.884047 & -0.883830 \\
7 & 1 & 0 & 2.647335 & -0.627800 & 0.000419 \\
8 & 1 & 0 & 1.823945 & 1.680440 & -0.002308 \\
9 & 1 & 0 & 1.449357 & -2.038049 & 0.000466 \\
10 & 6 & 0 & -2.000303 & 0.024247 & 0.000171 \\
11 & 1 & 0 & -2.590317 & -0.325080 & -0.865914 \\
12 & 1 & 0 & -2.590720 & -0.327548 & 0.864985 \\
13 & 1 & 0 & -1.939277 & 1.111996 & 0.001622 \\
14 & 1 & 0 & -0.753548 & -1.756758 & -0.000474
\end{tabular}

Energy: $\mathrm{HF}=-195.6402287$

Zero-Point Energy Correction: 0.124769

Imaginary Frequency: Minimum (No imaginary frequencies) 


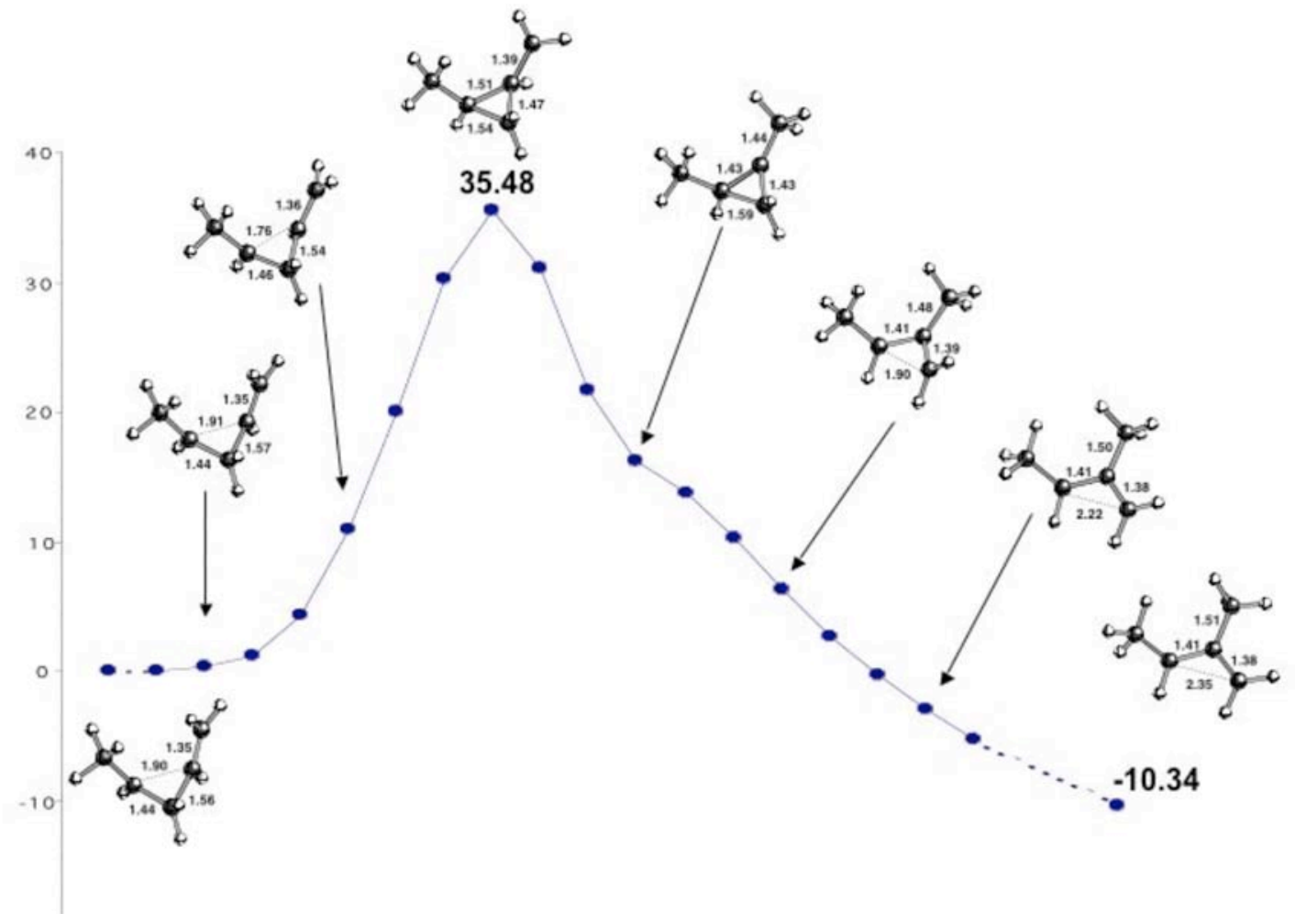


<smiles>CC1CCC1C</smiles>

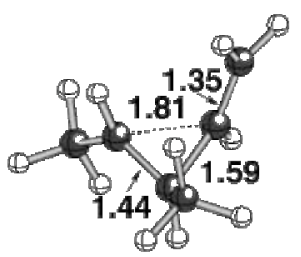

b3lyp/6-31G(d)

Coordinates:

Standard orientation:

\begin{tabular}{|c|c|c|c|c|}
\hline \multirow{2}{*}{$\begin{array}{l}\text { Center } \\
\text { Number }\end{array}$} & \multirow{2}{*}{$\begin{array}{l}\text { Atomic } \\
\text { Number }\end{array}$} & \multirow{2}{*}{$\begin{array}{l}\text { Atomic } \\
\text { Type }\end{array}$} & \multicolumn{2}{|c|}{ Coordinates (Angstror } \\
\hline & & & $\begin{array}{ll}X & Y\end{array}$ & $Y \quad Z$ \\
\hline 1 & 6 & 0.746262 & -0.289852 & -0.437091 \\
\hline 2 & 6 & -0.200253 & 0.895301 & 0.552990 \\
\hline 3 & 6 & -0.333676 & -0.686764 & 0.424160 \\
\hline 4 & 1 & -0.023180 & -1.095295 & 1.387265 \\
\hline 5 & 6 & -0.850855 & 1.773232 & -0.246637 \\
\hline 6 & 6 & -1.627820 & -1.261507 & -0.119870 \\
\hline 7 & 1 & -1.514449 & -2.346770 & -0.212185 \\
\hline 8 & 1 & -0.859924 & 2.835452 & -0.012767 \\
\hline 9 & 1 & -1.879400 & -0.870235 & -1.109712 \\
\hline 10 & 1 & 0.362204 & 1.243619 & 1.412895 \\
\hline 11 & 1 & -2.457548 & -1.062507 & 0.563216 \\
\hline 12 & 6 & 2.191232 & -0.279161 & -0.070992 \\
\hline 13 & 1 & 2.360932 & -0.350796 & 1.005993 \\
\hline 14 & 1 & 2.631809 & -1.172034 & -0.542980 \\
\hline 15 & 1 & 2.717273 & 0.587168 & -0.482533 \\
\hline 16 & 1 & 0.509491 & -0.142555 & -1.489672 \\
\hline 17 & 1 & -1.396551 & 1.466466 & -1.134879 \\
\hline
\end{tabular}

Energy: $\mathrm{HF}=-234.9473107$

Zero-Point Energy Correction: 0.154127

Imaginary Frequency: Minimum (No imaginary frequencies) 
<smiles>CC1CC2C=CC1C2C</smiles>

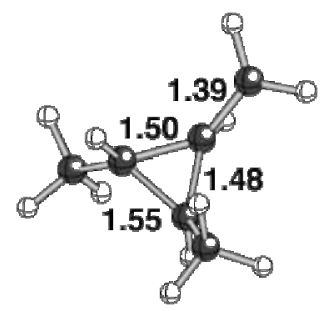

b3lyp/6-31G(d)

Coordinates:

Standard orientation:

\begin{tabular}{|c|c|c|c|c|c|}
\hline \multirow{2}{*}{$\begin{array}{l}\text { Center } \\
\text { Number }\end{array}$} & \multirow{2}{*}{$\begin{array}{l}\text { Atomic } \\
\text { Number }\end{array}$} & \multirow{2}{*}{\multicolumn{2}{|c|}{$\begin{array}{l}\text { Atomic } \\
\text { Type }\end{array}$}} & \multicolumn{2}{|c|}{ Coordinates (Angstro } \\
\hline & & & & X $\quad \mathrm{Y}$ & $\mathrm{Z}$ \\
\hline 1 & 6 & 0 & 0.800394 & -0.128603 & -0.443785 \\
\hline 2 & 6 & 0 & -0.222991 & 0.763903 & 0.194486 \\
\hline 3 & 6 & 0 & -0.358312 & -0.691020 & 0.427426 \\
\hline 4 & 1 & 0 & 0.008289 & -1.042820 & 1.392099 \\
\hline 5 & 6 & 0 & -1.009814 & 1.870166 & -0.098177 \\
\hline 6 & 6 & 0 & -1.525769 & -1.460929 & -0.148907 \\
\hline 7 & 1 & 0 & -1.250294 & -2.512557 & -0.273395 \\
\hline 8 & 1 & 0 & -0.590018 & 2.727980 & -0.628380 \\
\hline 9 & 1 & 0 & -1.825074 & -1.067221 & -1.127319 \\
\hline 10 & 1 & 0 & 0.120093 & 1.512770 & 1.035456 \\
\hline 11 & 1 & 0 & -2.394325 & -1.420449 & 0.516951 \\
\hline 12 & 6 & 0 & 2.230180 & -0.221541 & 0.041388 \\
\hline 13 & 1 & 0 & 2.299953 & -0.130393 & 1.130464 \\
\hline 14 & 1 & 0 & 2.653384 & -1.188012 & -0.248668 \\
\hline 15 & 1 & 0 & 2.845528 & 0.562847 & -0.411952 \\
\hline 16 & 1 & 0 & 0.670993 & -0.207772 & -1.524167 \\
\hline 17 & 1 & 0 & -2.020657 & 1.973772 & 0.304329 \\
\hline
\end{tabular}

Energy: $\mathrm{HF}=-234.8877988$

Zero-Point Energy Correction: 0.149347

Imaginary Frequency: One imaginary frequency $-667.0673 \mathrm{~cm}^{-1}$ 

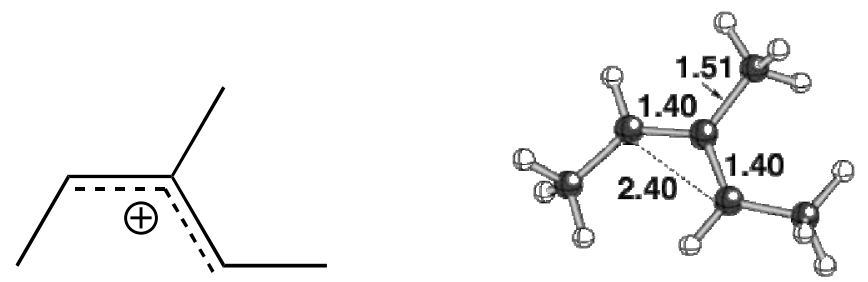

b3lyp/6-31G(d)

Coordinates:

Standard orientation:

\begin{tabular}{|c|c|c|c|c|}
\hline \multirow{2}{*}{$\begin{array}{l}\text { Center } \\
\text { Number }\end{array}$} & \multirow{2}{*}{$\begin{array}{l}\text { Atomic } \\
\text { Number }\end{array}$} & \multirow{2}{*}{$\begin{array}{r}\text { Atomic } \\
\text { Type }\end{array}$} & \multicolumn{2}{|c|}{ Coordinates (Angstroms) } \\
\hline & & & X $\quad Y$ & Z \\
\hline 1 & 6 & 1.385273 & 0.406270 & 0.000006 \\
\hline 2 & 6 & -0.013403 & 0.421016 & -0.000012 \\
\hline 3 & 6 & -0.686551 & -0.800874 & 0.000061 \\
\hline 4 & 1 & -0.094678 & -1.713611 & 0.000203 \\
\hline 5 & 6 & -0.759982 & 1.738958 & -0.000007 \\
\hline 6 & 6 & -2.141294 & -0.995748 & -0.000053 \\
\hline 7 & 1 & -2.418146 & -1.617586 & -0.867872 \\
\hline 8 & 1 & -0.067227 & 2.583689 & 0.000399 \\
\hline 9 & 1 & -2.725777 & -0.076507 & -0.000838 \\
\hline 10 & 1 & -1.398553 & 1.832543 & 0.884612 \\
\hline 11 & 1 & -2.418501 & -1.616149 & 0.868705 \\
\hline 12 & 6 & 2.338606 & -0.716776 & -0.000073 \\
\hline 13 & 1 & 1.910907 & -1.719014 & -0.001386 \\
\hline 14 & 1 & 3.006610 & -0.604867 & -0.868941 \\
\hline 15 & 1 & 3.004660 & -0.606372 & 0.870544 \\
\hline 16 & 1 & 1.862777 & 1.387878 & 0.000047 \\
\hline 17 & 1 & -1.397970 & 1.832926 & -0.885007 \\
\hline
\end{tabular}

Energy: $\mathrm{HF}=-234.9717542$

Zero-Point Energy Correction: $\mathbf{0 . 1 5 2 9 8 5}$

Imaginary Frequency: Minimum (No imaginary frequencies) 


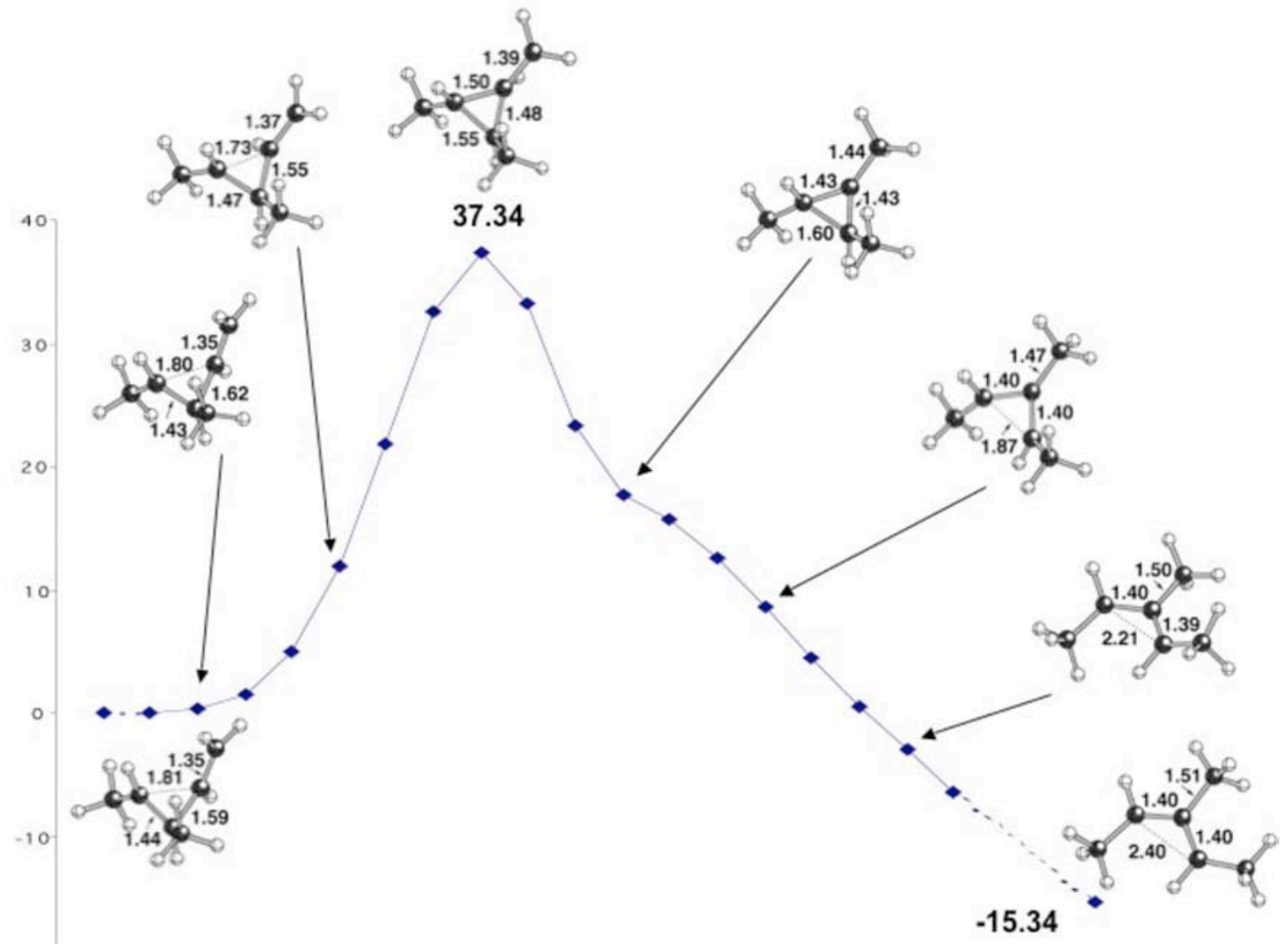



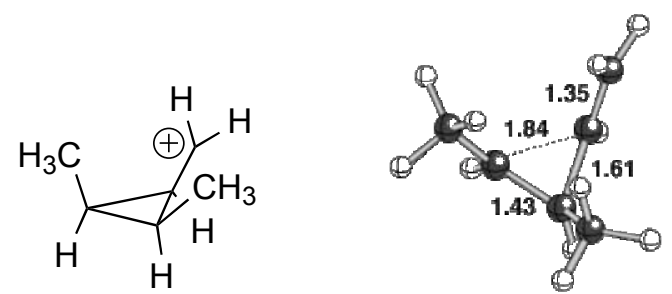

b3lyp/6-31G(d)

\section{Coordinates:}

Standard orientation:

\begin{tabular}{|c|c|c|c|c|}
\hline \multirow{2}{*}{$\begin{array}{l}\text { Center } \\
\text { Number }\end{array}$} & Atomic & \multirow{2}{*}{$\begin{array}{l}\text { Atomic } \\
\text { Type }\end{array}$} & \multicolumn{2}{|c|}{ Coordinates (Angstroms) } \\
\hline & Number & & X $\quad Y$ & Z \\
\hline 1 & 6 & -0.808285 & -0.276706 & 0.666638 \\
\hline 2 & 6 & 0.996227 & -0.587857 & 0.535096 \\
\hline 3 & 6 & 0.159427 & 0.781951 & 0.634067 \\
\hline 4 & 6 & 1.366001 & -1.159330 & -0.631005 \\
\hline 5 & 1 & 2.122484 & -1.940721 & -0.646558 \\
\hline 6 & 1 & 1.416111 & -0.930269 & 1.474781 \\
\hline 7 & 1 & 0.946318 & -0.871843 & -1.590819 \\
\hline 8 & 6 & 0.268993 & 1.792366 & -0.489971 \\
\hline 9 & 1 & -0.433182 & 2.609204 & -0.290600 \\
\hline 10 & 1 & 1.276840 & 2.214041 & -0.521733 \\
\hline 11 & 1 & 0.030940 & 1.382092 & -1.473647 \\
\hline 12 & 6 & -1.756686 & -0.663286 & -0.416293 \\
\hline 13 & 1 & -1.918842 & -1.743577 & -0.455852 \\
\hline 14 & 1 & -2.719651 & -0.208877 & -0.132815 \\
\hline 15 & 1 & -1.495462 & -0.280490 & -1.403509 \\
\hline 16 & 1 & -0.995931 & -0.739549 & 1.635498 \\
\hline 17 & 1 & 0.416307 & 1.187160 & 1.614057 \\
\hline
\end{tabular}

Energy: $\mathrm{HF}=-234.9428383$

Zero-Point Energy Correction: 0.154009

Imaginary Frequency: Minimum (No imaginary frequencies) 
<smiles>CC1CC2C=CC1C2C</smiles>

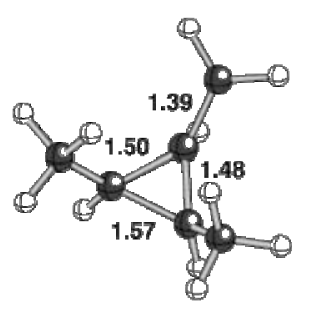

b3lyp/6-31G(d)

Coordinates:

Standard orientation:

\begin{tabular}{|c|c|c|c|c|c|}
\hline \multirow{2}{*}{$\begin{array}{l}\text { Center } \\
\text { Number }\end{array}$} & \multirow{2}{*}{$\begin{array}{l}\text { Atomic } \\
\text { Number }\end{array}$} & \multirow{2}{*}{\multicolumn{2}{|c|}{$\begin{array}{r}\text { Atomic } \\
\text { Type }\end{array}$}} & \multicolumn{2}{|c|}{ Coordinates (Angstroms) } \\
\hline & & & & X $\quad Y$ & $\mathrm{Z}$ \\
\hline 1 & 6 & 0 & -0.872763 & -0.093784 & 0.620701 \\
\hline 2 & 6 & 0 & 0.460515 & -0.744169 & 0.388692 \\
\hline 3 & 6 & 0 & 0.469500 & 0.712485 & 0.632203 \\
\hline 4 & 6 & 0 & 1.062218 & -1.663065 & -0.462024 \\
\hline 5 & 1 & 0 & 0.564550 & -2.603134 & -0.708830 \\
\hline 6 & 1 & 0 & 0.835473 & -1.541684 & 1.164486 \\
\hline 7 & 1 & 0 & 2.093799 & -1.537065 & -0.799087 \\
\hline 8 & 6 & 0 & 0.890484 & 1.679441 & -0.452471 \\
\hline 9 & 1 & 0 & 0.410703 & 2.649735 & -0.292922 \\
\hline 10 & 1 & 0 & 1.974044 & 1.836922 & -0.427039 \\
\hline 11 & 1 & 0 & 0.623616 & 1.325155 & -1.453660 \\
\hline 12 & 6 & 0 & -1.908483 & -0.037796 & -0.477796 \\
\hline 13 & 1 & 0 & -2.520573 & -0.945993 & -0.483500 \\
\hline 14 & 1 & 0 & -2.579653 & 0.808527 & -0.296749 \\
\hline 15 & 1 & 0 & -1.462163 & 0.081269 & -1.469543 \\
\hline 16 & 1 & 0 & -1.275201 & -0.209721 & 1.625033 \\
\hline 17 & 1 & 0 & 0.726579 & 1.017329 & 1.645976 \\
\hline
\end{tabular}

Energy: $\mathrm{HF}=-234.8872791$

Zero-Point Energy Correction: 0.149582

Imaginary Frequency: One imaginary frequency $-700.7484 \mathrm{~cm}^{-1}$ 

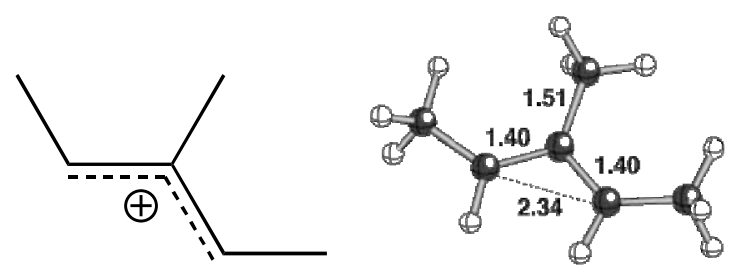

b3lyp/6-31G(d)

\section{Coordinates:}

Standard orientation:

\begin{tabular}{|c|c|c|c|c|}
\hline \multirow{2}{*}{$\begin{array}{l}\text { Center } \\
\text { Number }\end{array}$} & \multirow{2}{*}{$\begin{array}{l}\text { Atomic } \\
\text { Number }\end{array}$} & \multirow{2}{*}{$\begin{array}{r}\text { Atomic } \\
\text { Type }\end{array}$} & \multicolumn{2}{|c|}{ Coordinates (Angstroms) } \\
\hline & & & X $\quad Y$ & $\mathrm{Z}$ \\
\hline 1 & 6 & 1.185698 & -0.689082 & -0.000078 \\
\hline 2 & 6 & 0.004765 & 0.056753 & -0.000134 \\
\hline 3 & 6 & -1.155369 & -0.722302 & -0.000105 \\
\hline 4 & 6 & -0.051915 & 1.566031 & -0.000065 \\
\hline 5 & 1 & 0.941087 & 2.015667 & -0.001425 \\
\hline 6 & 1 & -0.580597 & 1.934877 & 0.885200 \\
\hline 7 & 1 & -0.582968 & 1.934892 & -0.883888 \\
\hline 8 & 6 & -2.541983 & -0.241023 & 0.000134 \\
\hline 9 & 1 & -3.068937 & -0.669372 & -0.868570 \\
\hline 10 & 1 & -3.068510 & -0.669163 & 0.869204 \\
\hline 11 & 1 & -2.648604 & 0.843374 & 0.000066 \\
\hline 12 & 6 & 2.572454 & -0.206295 & 0.000147 \\
\hline 13 & 1 & 3.097057 & -0.636012 & 0.869340 \\
\hline 14 & 1 & 3.097075 & -0.635358 & -0.869369 \\
\hline 15 & 1 & 2.686975 & 0.876754 & 0.000539 \\
\hline 16 & 1 & 1.077355 & -1.774359 & -0.000242 \\
\hline 17 & 1 & -1.031830 & -1.805788 & -0.000244 \\
\hline
\end{tabular}

Energy: $\mathrm{HF}=-234.9761632$

Zero-Point Energy Correction: 0.152996

Imaginary Frequency: Minimum (No imaginary frequencies) 


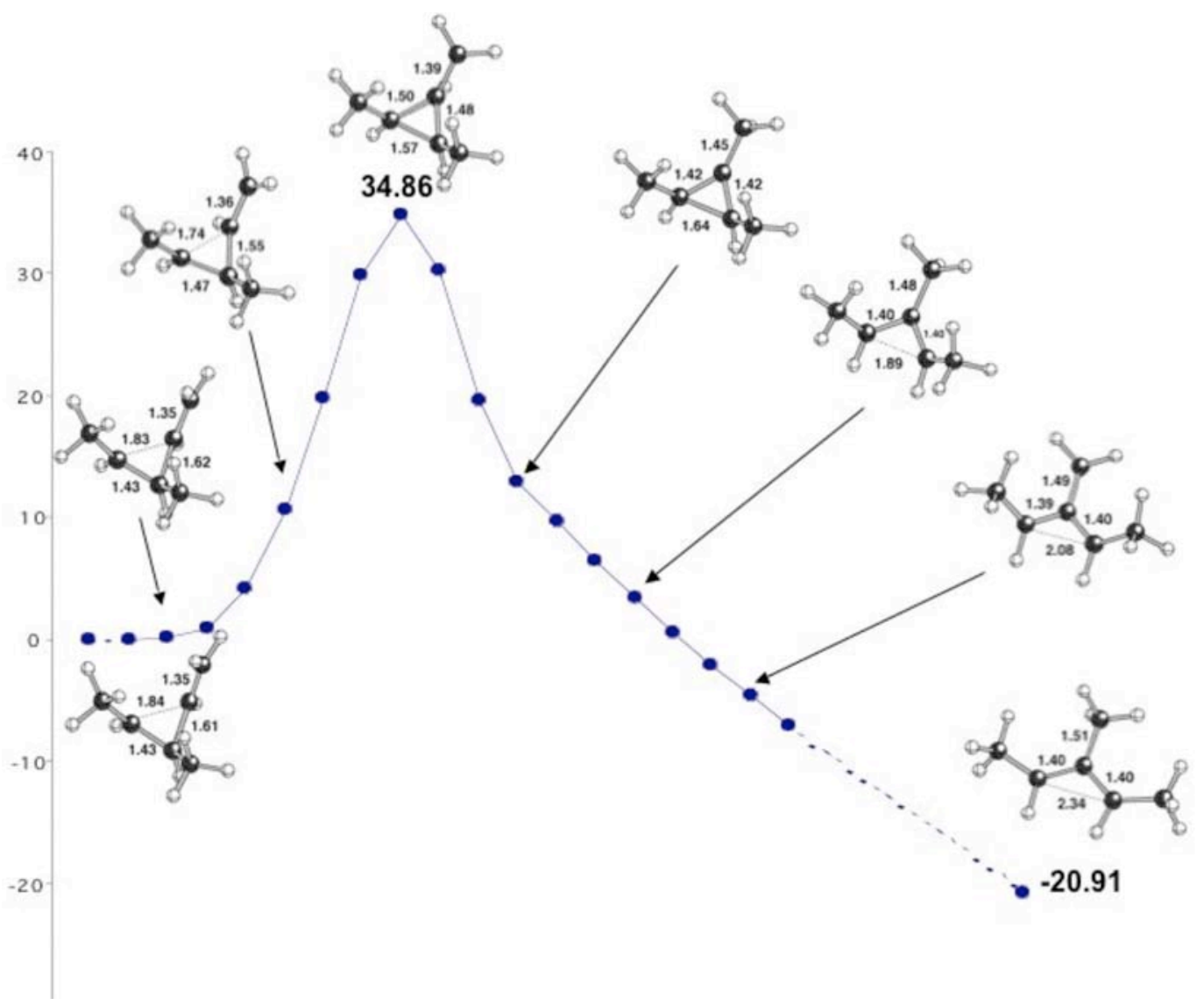


<smiles>CC1C=CC1C</smiles>

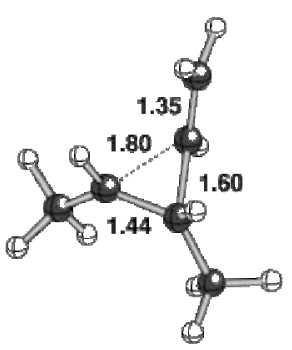

b3lyp/6-31G(d)

\section{Coordinates:}

Standard orientation:

\begin{tabular}{|c|c|c|c|c|}
\hline \multirow{2}{*}{$\begin{array}{l}\text { Center } \\
\text { Number }\end{array}$} & \multirow{2}{*}{$\begin{array}{l}\text { Atomic } \\
\text { Number }\end{array}$} & \multirow{2}{*}{$\begin{array}{l}\text { Atomic } \\
\text { Type }\end{array}$} & \multicolumn{2}{|c|}{ Coordinates (Angstroms } \\
\hline & & & $\mathrm{X} \quad \mathrm{Y}$ & $\mathrm{Z}$ \\
\hline 1 & 6 & -0.205771 & 0.735562 & 0.565607 \\
\hline 2 & 6 & 0.924830 & -0.207471 & -0.467574 \\
\hline 3 & 6 & -0.242200 & -0.699805 & 0.511827 \\
\hline 4 & 6 & 2.212275 & -0.084754 & -0.070346 \\
\hline 5 & 1 & 3.014563 & 0.029612 & -0.796091 \\
\hline 6 & 1 & 0.649421 & -0.155552 & -1.515951 \\
\hline 7 & 6 & -1.041181 & 1.684390 & -0.227458 \\
\hline 8 & 1 & -1.842224 & 2.022628 & 0.449125 \\
\hline 9 & 1 & -0.476782 & 2.574694 & -0.518899 \\
\hline 10 & 1 & -1.512508 & 1.236623 & -1.103674 \\
\hline 11 & 6 & -1.326630 & -1.501754 & -0.183029 \\
\hline 12 & 1 & -0.949758 & -2.492400 & -0.449500 \\
\hline 13 & 1 & -2.163105 & -1.631934 & 0.512368 \\
\hline 14 & 1 & -1.705975 & -1.018527 & -1.086548 \\
\hline 15 & 1 & 0.364753 & 1.179130 & 1.378447 \\
\hline 16 & 1 & 0.192697 & -1.196462 & 1.378134 \\
\hline 17 & 1 & 2.500979 & -0.104821 & 0.978426 \\
\hline
\end{tabular}

Energy: $\mathrm{HF}=-234.9471046$

Zero-Point Energy Correction: $\mathbf{0 . 1 5 4 0 6 0}$

Imaginary Frequency: Minimum (No imaginary frequencies) 

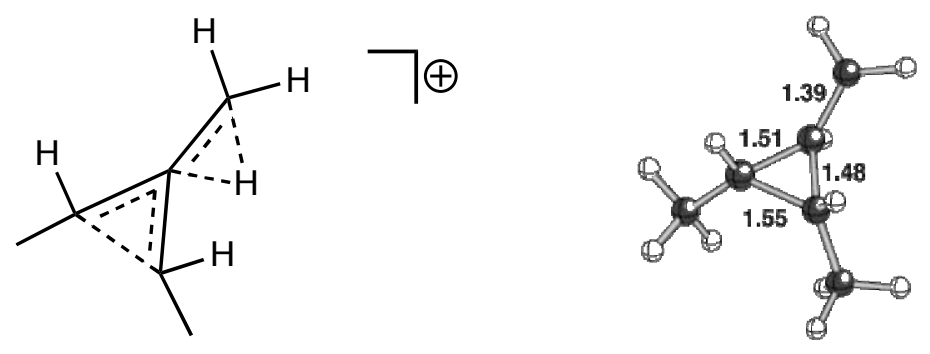

b3lyp/6-31G(d)

\section{Coordinates:}

Standard orientation:

\begin{tabular}{|c|c|c|c|c|}
\hline \multirow{2}{*}{$\begin{array}{l}\text { Center } \\
\text { Number }\end{array}$} & \multirow{2}{*}{$\begin{array}{l}\text { Atomic } \\
\text { Number }\end{array}$} & \multirow{2}{*}{$\begin{array}{r}\text { Atomic } \\
\text { Type }\end{array}$} & \multicolumn{2}{|c|}{ Coordinates (Angstroms) } \\
\hline & & & X $\quad Y$ & Z Z \\
\hline 1 & 6 & 0.285172 & -0.766314 & 0.544734 \\
\hline 2 & 6 & -0.901608 & -0.051901 & -0.053170 \\
\hline 3 & 6 & 0.167317 & 0.778635 & 0.548384 \\
\hline 4 & 6 & -2.279839 & -0.108348 & -0.186766 \\
\hline 5 & 1 & -2.779921 & -1.053479 & -0.411682 \\
\hline 6 & 1 & -1.038358 & -0.148106 & -1.224436 \\
\hline 7 & 6 & 1.315963 & -1.512318 & -0.275777 \\
\hline 8 & 1 & 2.252114 & -1.572732 & 0.287866 \\
\hline 9 & 1 & 0.977597 & -2.535604 & -0.468453 \\
\hline 10 & 1 & 1.526370 & -1.033548 & -1.235433 \\
\hline 11 & 6 & 1.089789 & 1.649617 & -0.287312 \\
\hline 12 & 1 & 0.640101 & 2.637753 & -0.427762 \\
\hline 13 & 1 & 2.044300 & 1.782236 & 0.229810 \\
\hline 14 & 1 & 1.291985 & 1.227908 & -1.275877 \\
\hline 15 & 1 & 0.027483 & -1.256852 & 1.483990 \\
\hline 16 & 1 & -0.113072 & 1.217054 & 1.506550 \\
\hline 17 & 1 & -2.889354 & 0.799137 & -0.205123 \\
\hline
\end{tabular}

Energy: $\mathrm{HF}=-234.8848332$

Zero-Point Energy Correction: 0.150002

Imaginary Frequency: One imaginary frequency $-746.5452 \mathrm{~cm}^{-1}$ 

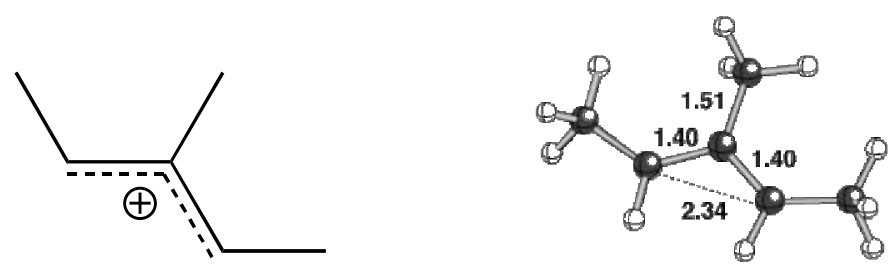

b31yp/6-31G(d)

\section{Coordinates:}

Standard orientation:

\begin{tabular}{|c|c|c|c|c|}
\hline \multirow{2}{*}{$\begin{array}{l}\text { Center } \\
\text { Number }\end{array}$} & \multirow{2}{*}{$\begin{array}{l}\text { Atomic } \\
\text { Number }\end{array}$} & \multirow{2}{*}{$\begin{array}{r}\text { Atomic } \\
\text { Type }\end{array}$} & \multicolumn{2}{|c|}{ Coordinates (Angstroms) } \\
\hline & & & X $\quad Y$ & I $\quad \mathrm{Z}$ \\
\hline 1 & 6 & -1.185703 & -0.689076 & 0.000117 \\
\hline 2 & 6 & -0.004784 & 0.056776 & 0.000006 \\
\hline 3 & 6 & 1.155345 & -0.722304 & -0.000140 \\
\hline 4 & 6 & 0.051953 & 1.566027 & 0.000012 \\
\hline 5 & 1 & -0.940999 & 2.015773 & 0.000494 \\
\hline 6 & 1 & 0.581426 & 1.934871 & -0.884788 \\
\hline 7 & 6 & -2.572452 & -0.206299 & 0.000031 \\
\hline 8 & 1 & -3.096392 & -0.634409 & -0.870411 \\
\hline 9 & 1 & -3.097763 & -0.636918 & 0.868285 \\
\hline 10 & 1 & -2.686969 & 0.876759 & 0.001349 \\
\hline 11 & 6 & 2.541969 & -0.241054 & -0.000080 \\
\hline 12 & 1 & 3.067800 & -0.667627 & 0.870214 \\
\hline 13 & 1 & 3.069617 & -0.670951 & -0.867545 \\
\hline 14 & 1 & 2.648623 & 0.843345 & -0.001763 \\
\hline 15 & 1 & -1.077313 & -1.774342 & 0.000176 \\
\hline 16 & 1 & 1.031764 & -1.805779 & 0.000011 \\
\hline 17 & 1 & 0.582238 & 1.934860 & 0.884305 \\
\hline
\end{tabular}

Energy: $\mathrm{HF}=-234.9761632$

Zero-Point Energy Correction: 0.152995

Imaginary Frequency: Minimum (No imaginary frequencies) 


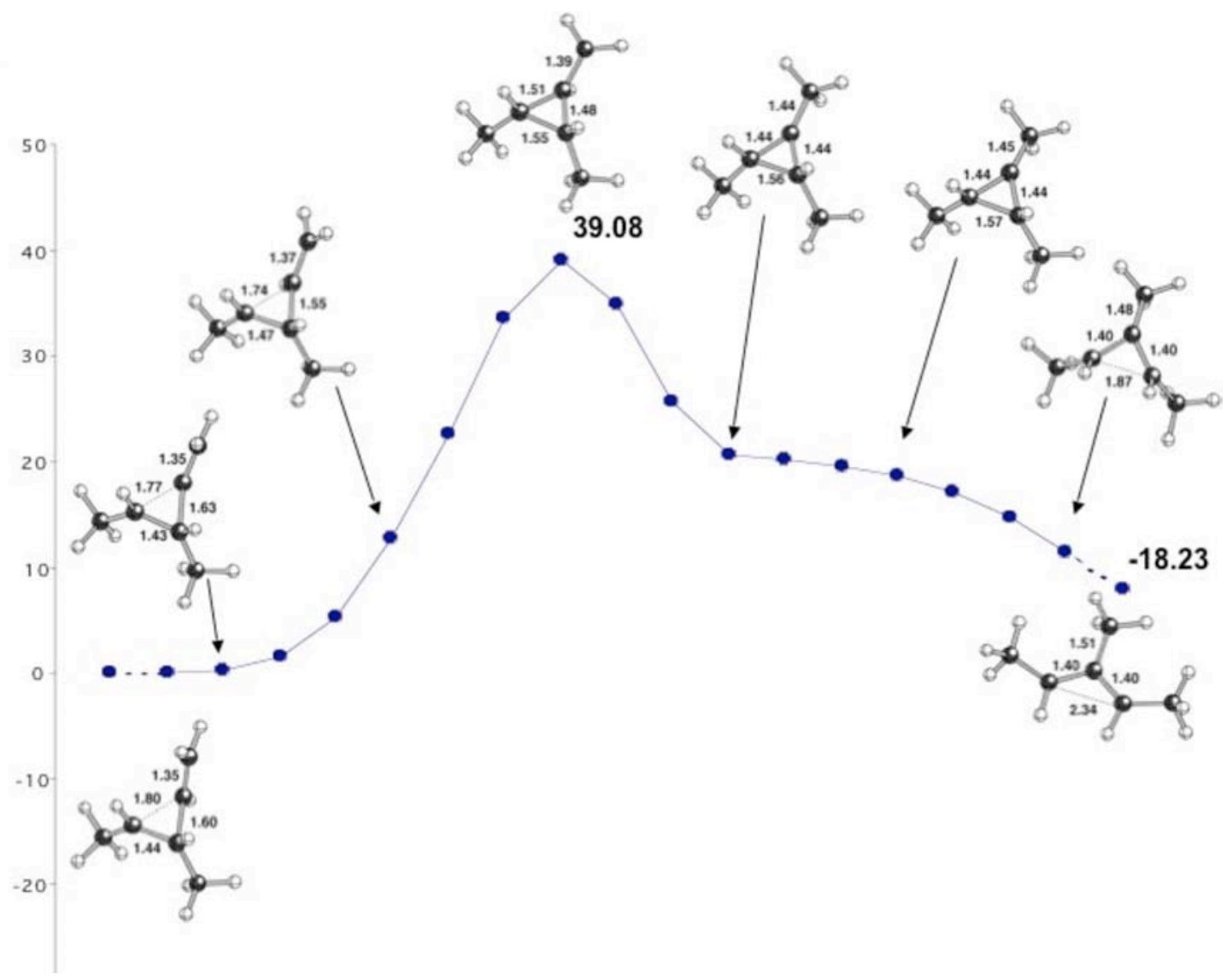




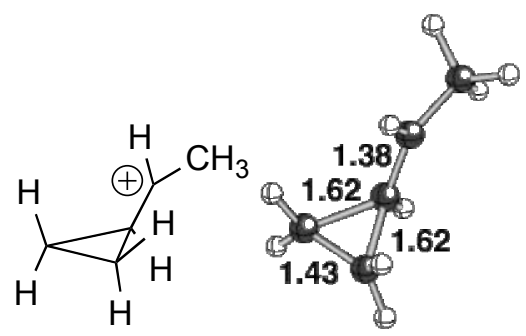

b3lyp/6-31G(d)

\section{Coordinates:}

Standard orientation:

\begin{tabular}{|c|c|c|c|c|c|}
\hline \multirow{2}{*}{$\begin{array}{l}\text { Center } \\
\text { Number }\end{array}$} & \multirow{2}{*}{\multicolumn{2}{|c|}{$\begin{array}{l}\text { Atomic } \\
\text { Number }\end{array}$}} & & \multicolumn{2}{|c|}{ Coordinates (Angstroms } \\
\hline & & & & $\mathrm{X} \quad \mathrm{Y}$ & Y Z \\
\hline 1 & 6 & 0 & -1.582052 & -0.716035 & -0.090552 \\
\hline 2 & 6 & 0 & -0.234068 & -0.000245 & 0.462053 \\
\hline 3 & 6 & 0 & -1.581650 & 0.716184 & -0.090291 \\
\hline 4 & 6 & 0 & 0.870856 & 0.000047 & -0.362110 \\
\hline 5 & 1 & 0 & -0.111446 & -0.000405 & 1.539599 \\
\hline 6 & 1 & 0 & -1.457817 & -1.263162 & -1.019414 \\
\hline 7 & 1 & 0 & -1.457724 & 1.263968 & -1.018816 \\
\hline 8 & 1 & 0 & 0.702455 & 0.000153 & -1.441362 \\
\hline 9 & 1 & 0 & -2.049020 & 1.264879 & 0.722880 \\
\hline 10 & 6 & 0 & 2.270758 & 0.000051 & 0.085133 \\
\hline 11 & 1 & 0 & 2.787328 & 0.870044 & -0.351133 \\
\hline 12 & 1 & 0 & 2.386493 & 0.000683 & 1.170060 \\
\hline 13 & 1 & 0 & 2.786309 & -0.871397 & -0.349641 \\
\hline 14 & 1 & 0 & -2.049641 & -1.264772 & 0.722429 \\
\hline
\end{tabular}

Energy: $\mathrm{HF}=-195.635271$

Zero-Point Energy Correction: 0.126063

Imaginary Frequency: Minimum (No imaginary frequencies) 
<smiles>CC1=CC=CC1C</smiles>

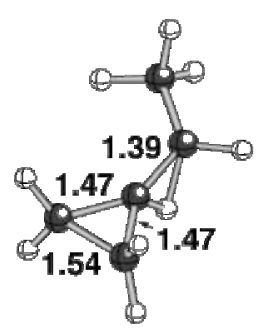

b3lyp/6-31G(d)

\section{Coordinates:}

Standard orientation:

\begin{tabular}{cccccc} 
Center & \multicolumn{2}{c}{ Atomic } & \multicolumn{2}{c}{ Atomic } & \multicolumn{2}{c}{ Coordinates } \\
Number & Number & Type & X & Y & Z \\
\hline------------ \\
1 & 6 & 0 & -1.293308 & 0.932234 & -0.004319 \\
2 & 6 & 0 & -0.346422 & -0.189282 & -0.072144 \\
3 & 6 & 0 & -1.766280 & -0.528300 & 0.101532 \\
4 & 6 & 0 & 0.972713 & -0.603541 & 0.010230 \\
5 & 1 & 0 & 0.278831 & -0.498606 & -1.154035 \\
6 & 1 & 0 & -1.247873 & 1.556728 & 0.885999 \\
7 & 1 & 0 & -2.052537 & -0.941474 & 1.066984 \\
8 & 1 & 0 & 1.143553 & -1.680883 & 0.086805 \\
9 & 1 & 0 & -2.323972 & -0.923180 & -0.744338 \\
10 & 6 & 0 & 2.157160 & 0.303380 & 0.047266 \\
11 & 1 & 0 & 2.939861 & -0.030669 & -0.640643 \\
12 & 1 & 0 & 1.896624 & 1.346773 & -0.140440 \\
13 & 1 & 0 & 2.575133 & 0.221631 & 1.061835 \\
14 & 1 & 0 & -1.552802 & 1.462732 & -0.917566 \\
------------------------------------------------------------
\end{tabular}

Energy: $H F=-195.5852075$

Zero-Point Energy Correction: 0.122033

Imaginary Frequency: One imaginary frequency $-416.2175 \mathrm{~cm}^{-1}$ 

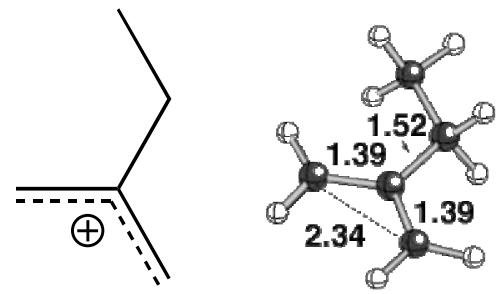

b31yp/6-31G(d)

Coordinates:

Standard orientation:

\begin{tabular}{|c|c|c|c|c|c|}
\hline \multirow{2}{*}{$\begin{array}{l}\text { Center } \\
\text { Number }\end{array}$} & \multirow{2}{*}{$\begin{array}{l}\text { Atomic } \\
\text { Number }\end{array}$} & \multirow{2}{*}{\multicolumn{2}{|c|}{$\begin{array}{l}\text { Atomic } \\
\text { Type }\end{array}$}} & \multicolumn{2}{|c|}{ Coordinates (Angstroms) } \\
\hline & & & & X $\quad Y$ & K $\quad \mathrm{Z}$ \\
\hline 1 & 6 & 0 & -0.701337 & 1.367946 & 0.000080 \\
\hline 2 & 6 & 0 & -0.588892 & -0.018326 & 0.000091 \\
\hline 3 & 6 & 0 & -1.808043 & -0.687730 & -0.000161 \\
\hline 4 & 6 & 0 & 0.730265 & -0.770798 & 0.000248 \\
\hline 5 & 1 & 0 & 0.732692 & -1.433924 & -0.874777 \\
\hline 6 & 1 & 0 & 0.172589 & 2.015060 & 0.000093 \\
\hline 7 & 1 & 0 & -1.860972 & -1.776190 & -0.000334 \\
\hline 8 & 1 & 0 & 0.732848 & -1.433213 & 0.875818 \\
\hline 9 & 1 & 0 & -2.755930 & -0.153042 & -0.000210 \\
\hline 10 & 6 & 0 & 1.984282 & 0.104643 & -0.000203 \\
\hline 11 & 1 & 0 & 2.871916 & -0.532587 & -0.000220 \\
\hline 12 & 1 & 0 & 2.040869 & 0.740842 & -0.891301 \\
\hline 13 & 1 & 0 & 2.041217 & 0.741269 & 0.890566 \\
\hline 14 & 1 & 0 & -1.672876 & 1.857369 & 0.000034 \\
\hline
\end{tabular}

Energy: $\mathrm{HF}=-195.613343$

Zero-Point Energy Correction: 0.125519

Imaginary Frequency: Minimum (No imaginary frequencies) 


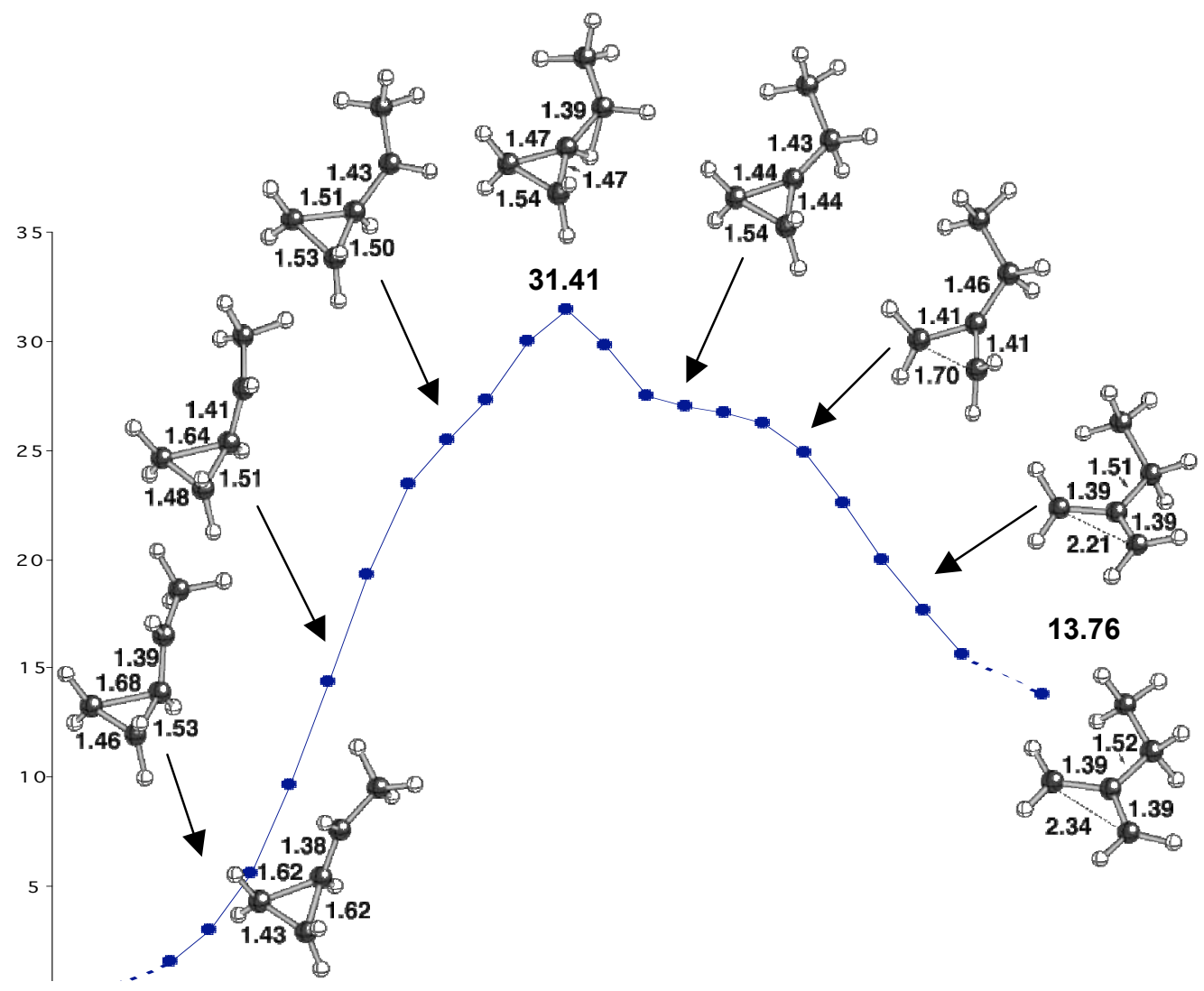

S65 

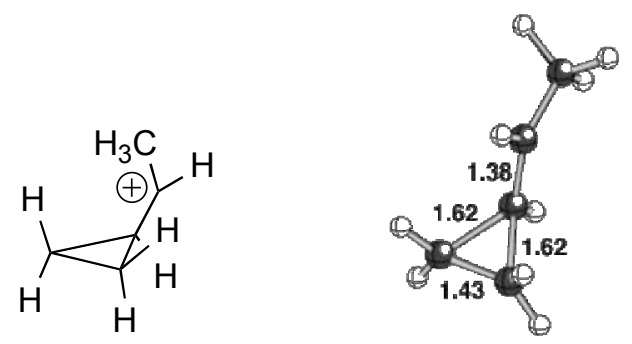

b3lyp/6-31G(d)

\section{Coordinates:}

Standard orientation:

\begin{tabular}{|c|c|c|c|c|}
\hline \multirow{2}{*}{$\begin{array}{l}\text { Center } \\
\text { Number }\end{array}$} & \multirow{2}{*}{$\begin{array}{l}\text { Atomic } \\
\text { Number }\end{array}$} & \multirow{2}{*}{$\begin{array}{r}\text { Atomic } \\
\text { Type }\end{array}$} & \multicolumn{2}{|c|}{ Coordinates (Angstroms } \\
\hline & & & $\begin{array}{ll}X & Y\end{array}$ & Y $\quad Z$ \\
\hline 1 & 6 & 1.581719 & 0.716171 & -0.090185 \\
\hline 2 & 6 & 0.234049 & -0.000274 & 0.461990 \\
\hline 3 & 6 & 1.582035 & -0.716045 & -0.090603 \\
\hline 4 & 6 & -0.870885 & 0.000135 & -0.362150 \\
\hline 5 & 1 & 0.111479 & -0.000581 & 1.539543 \\
\hline 6 & 1 & 1.457824 & 1.263995 & -1.018694 \\
\hline 7 & 1 & 1.457883 & -1.263058 & -1.019544 \\
\hline 8 & 1 & 2.049505 & -1.264915 & 0.722358 \\
\hline 9 & 1 & 2.049043 & 1.264821 & 0.723038 \\
\hline 10 & 6 & -2.270764 & 0.000013 & 0.085162 \\
\hline 11 & 1 & -2.786422 & -0.871163 & -0.350017 \\
\hline 12 & 1 & -2.386454 & 0.000171 & 1.170094 \\
\hline 13 & 1 & -2.787258 & 0.870288 & -0.350652 \\
\hline 14 & 1 & -0.702525 & 0.000449 & -1.441407 \\
\hline
\end{tabular}

Energy: $\mathrm{HF}=-195.635271$

Zero-Point Energy Correction: 0.126063 Imaginary Frequency: Minimum (No imaginary frequencies) 


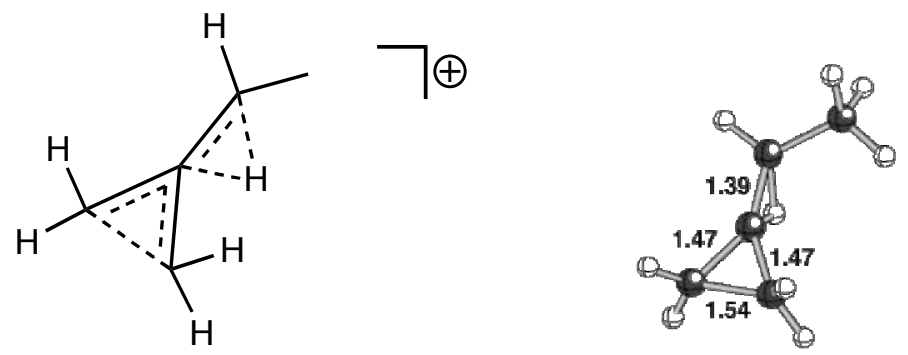

b3lyp/6-31G(d)

Coordinates:

Standard orientation:

\begin{tabular}{|c|c|c|c|c|}
\hline \multirow{2}{*}{$\begin{array}{l}\text { Center } \\
\text { Number }\end{array}$} & \multirow{2}{*}{$\begin{array}{l}\text { Atomic } \\
\text { Number }\end{array}$} & \multirow{2}{*}{$\begin{array}{r}\text { Atomic } \\
\quad \text { Type }\end{array}$} & \multicolumn{2}{|c|}{ Coordinates (Angstro1 } \\
\hline & & & X $\quad Y$ & $Y \quad Z$ \\
\hline 1 & 6 & 1.766086 & -0.528466 & 0.101993 \\
\hline 2 & 6 & 14 & -0.189185 & -0.072952 \\
\hline 3 & 6 & 07 & 0.932221 & -0.004280 \\
\hline 4 & 6 & -0.972734 & -0.603376 & 0.010058 \\
\hline 5 & 1 & -0.277 & -0.498423 & -1.154729 \\
\hline 6 & 1 & 2.0514 & -0.941701 & 1.067659 \\
\hline 7 & 1 & & 1.556922 & 5937 \\
\hline 8 & 1 & 05 & 1.462524 & -0.917416 \\
\hline 9 & 1 & 2.324545 & -0.923174 & -0.743444 \\
\hline 10 & 6 & -2.157174 & 0.303349 & 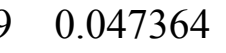 \\
\hline 11 & 1 & -2.574306 & 0.221253 & 1.062401 \\
\hline 12 & 1 & -1.896 & 1.346838 & $8-0.140173$ \\
\hline 13 & 1 & -2.940 & -0.030772 & $2-0.639766$ \\
\hline 14 & 1 & -1.143616 & -1.680723 & 0.086432 \\
\hline
\end{tabular}

Energy: $\mathrm{HF}=-195.5852074$

Zero-Point Energy Correction: 0.122032

Imaginary Frequency: One imaginary frequency $-417.4378 \mathrm{~cm}^{-1}$ 

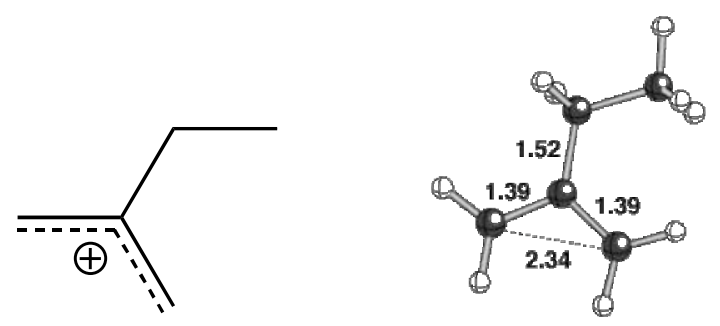

b3lyp/6-31G(d)

Coordinates:

Standard orientation:

\begin{tabular}{|c|c|c|c|c|c|}
\hline \multirow{2}{*}{$\begin{array}{l}\text { Center } \\
\text { Number }\end{array}$} & \multirow{2}{*}{$\begin{array}{l}\text { Atomic } \\
\text { Number }\end{array}$} & \multirow{2}{*}{\multicolumn{2}{|c|}{$\begin{array}{r}\text { Atomic } \\
\text { Type }\end{array}$}} & \multicolumn{2}{|c|}{ Coordinates (Angstroms) } \\
\hline & & & & $\begin{array}{ll}X & Y\end{array}$ & Z \\
\hline 1 & 6 & 0 & -1.807993 & -0.687741 & -0.000152 \\
\hline 2 & 6 & 0 & -0.588864 & -0.018323 & 0.000090 \\
\hline 3 & 6 & 0 & -0.701374 & 1.367919 & 0.000079 \\
\hline 4 & 6 & 0 & 0.730266 & -0.770779 & 0.000211 \\
\hline 5 & 1 & 0 & 0.732811 & -1.433308 & 0.875689 \\
\hline 6 & 1 & 0 & -1.860884 & -1.776209 & -0.000157 \\
\hline 7 & 1 & 0 & 0.172457 & 2.015164 & 0.000102 \\
\hline 8 & 1 & 0 & -1.672968 & 1.857233 & 0.000026 \\
\hline 9 & 1 & 0 & -2.755896 & -0.153088 & -0.000273 \\
\hline 10 & 6 & 0 & 1.984270 & 0.104665 & -0.000179 \\
\hline 11 & 1 & 0 & 2.040902 & 0.740798 & -0.891321 \\
\hline 12 & 1 & 0 & 2.041121 & 0.741355 & 0.890554 \\
\hline 13 & 1 & 0 & 2.871908 & -0.532557 & -0.000044 \\
\hline 14 & 1 & 0 & 0.732717 & -1.433840 & -0.874869 \\
\hline
\end{tabular}

Energy: $H F=-195.613343$

Zero-Point Energy Correction: 0.125520

Imaginary Frequency: Minimum (No imaginary frequencies) 


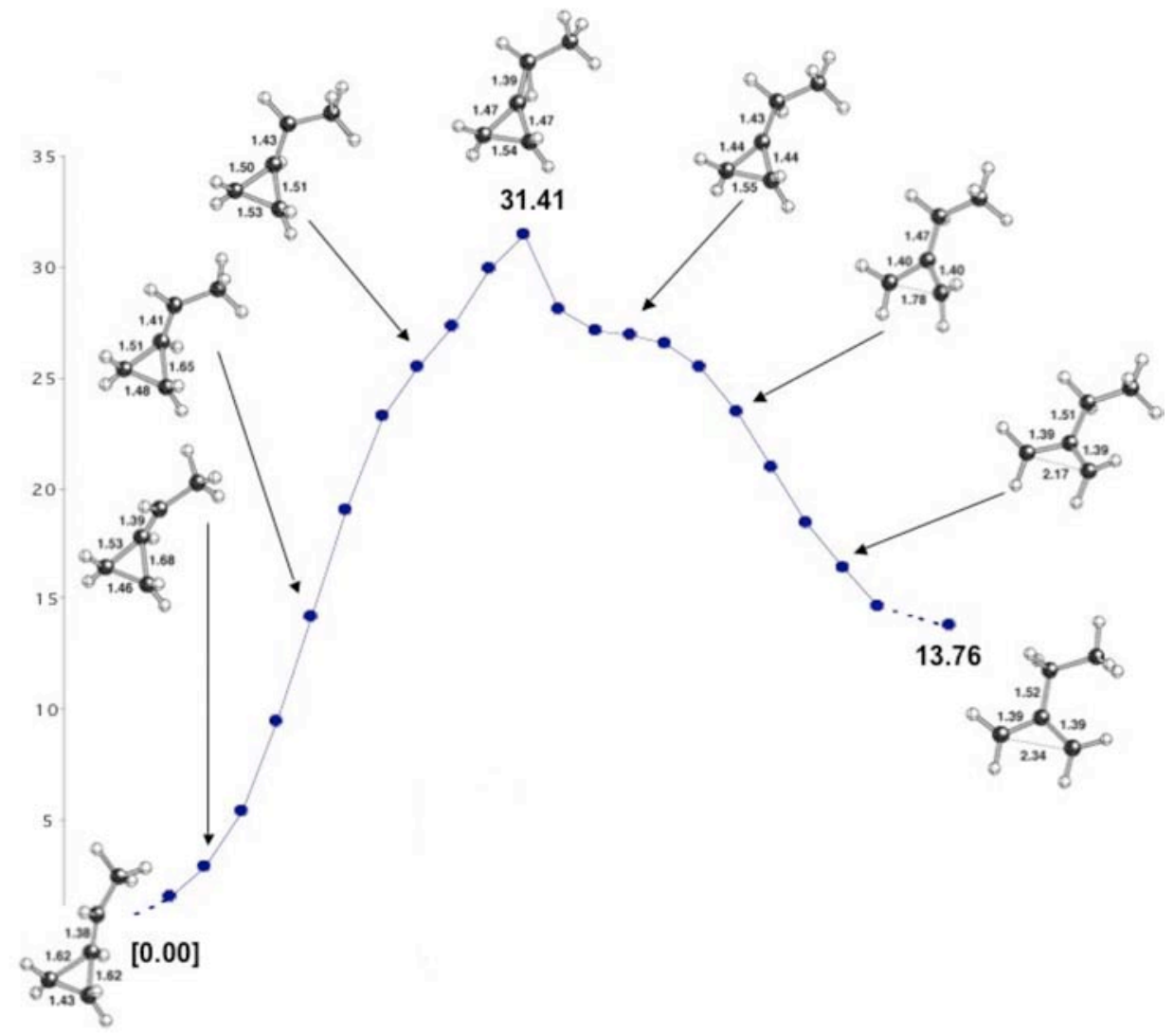


b3lyp/6-31G(d)

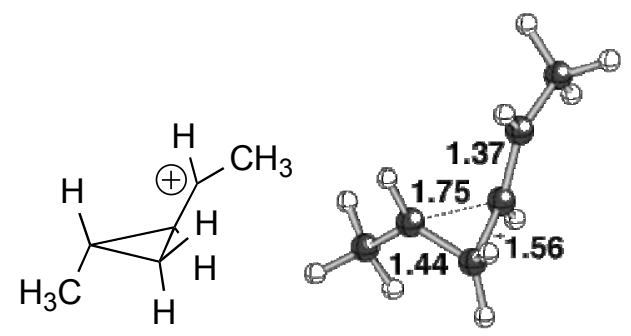

\section{Coordinates:}

Standard orientation:

\begin{tabular}{|c|c|c|c|c|}
\hline \multirow{2}{*}{$\begin{array}{l}\text { Center } \\
\text { Number }\end{array}$} & \multirow{2}{*}{$\begin{array}{l}\text { Atomic } \\
\text { Number }\end{array}$} & \multirow{2}{*}{$\begin{array}{r}\text { Atomic } \\
\text { Type }\end{array}$} & \multicolumn{2}{|c|}{ Coordinates (Angstroms) } \\
\hline & & & $\begin{array}{ll}X & Y\end{array}$ & $\mathrm{Z}$ \\
\hline 1 & 6 & -1.200594 & 0.033792 & -0.494223 \\
\hline 2 & 6 & 0.230047 & 0.304369 & 0.478038 \\
\hline 3 & 6 & -0.926752 & 1.295210 & 0.150499 \\
\hline 4 & 6 & 1.360008 & 0.214249 & -0.294888 \\
\hline 5 & 1 & 0.183120 & -0.203761 & 1.435724 \\
\hline 6 & 6 & -2.091583 & -1.032100 & 0.061802 \\
\hline 7 & 1 & -3.060279 & -0.933524 & -0.450360 \\
\hline 8 & 1 & -1.715387 & -2.036650 & -0.151867 \\
\hline 9 & 1 & -2.266245 & -0.920373 & 1.134946 \\
\hline 10 & 1 & -0.938074 & -0.056043 & -1.545335 \\
\hline 11 & 1 & -0.636824 & 2.145742 & -0.460343 \\
\hline 12 & 1 & 1.366056 & 0.723905 & -1.260391 \\
\hline 13 & 1 & -1.490236 & 1.547519 & 1.046395 \\
\hline 14 & 6 & 2.589092 & -0.515614 & 0.072225 \\
\hline 15 & 1 & 3.430288 & 0.195040 & 0.089593 \\
\hline 16 & 1 & 2.521727 & -1.022285 & 1.036729 \\
\hline 17 & 1 & 2.844537 & -1.238999 & -0.715808 \\
\hline
\end{tabular}

Energy: $\mathrm{HF}=-234.9612055$

Zero-Point Energy Correction: 0.154404 Imaginary Frequency: Minimum (No imaginary frequencies) 


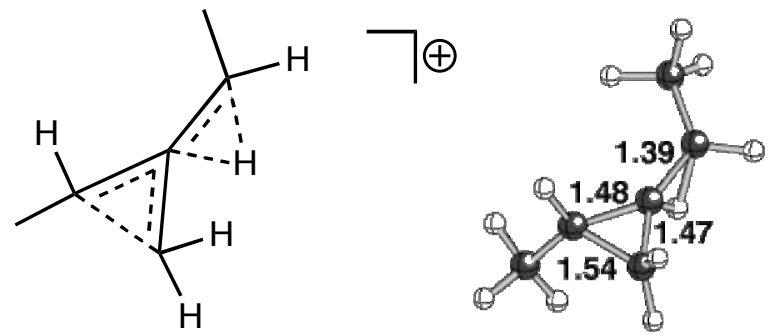

b3lyp/6-31G(d)

\section{Coordinates:}

Standard orientation:

\begin{tabular}{|c|c|c|c|c|}
\hline \multirow{2}{*}{$\begin{array}{l}\text { Center } \\
\text { Number }\end{array}$} & \multirow{2}{*}{$\begin{array}{l}\text { Atomic } \\
\text { Number }\end{array}$} & \multirow{2}{*}{$\begin{array}{r}\text { Atomic } \\
\text { Type }\end{array}$} & \multicolumn{2}{|c|}{ Coordinates (Angstroms } \\
\hline & & & X $\quad Y$ & Z Z \\
\hline 1 & 6 & 1.083895 & -0.119510 & 0.510384 \\
\hline 2 & 6 & -0.124315 & 0.506552 & -0.060944 \\
\hline 3 & 6 & 1.082046 & 1.345974 & 0.033480 \\
\hline 4 & 6 & -1.501348 & 0.471272 & -0.213312 \\
\hline 5 & 1 & -0.579107 & 0.172119 & -1.200159 \\
\hline 6 & 6 & 1.898507 & -1.160348 & -0.241425 \\
\hline 7 & 1 & 2.927815 & -1.152271 & 0.127680 \\
\hline 8 & 1 & 1.488356 & -2.162834 & -0.086460 \\
\hline 9 & 1 & 1.922204 & -0.955897 & -1.317385 \\
\hline 10 & 1 & 1.015516 & -0.278445 & 1.587581 \\
\hline 11 & 1 & 1.055607 & 2.163590 & 0.751456 \\
\hline 12 & 1 & -1.976692 & 1.377772 & -0.599094 \\
\hline 13 & 1 & 1.643147 & 1.558064 & -0.874405 \\
\hline 14 & 6 & -2.376044 & -0.687648 & 0.123209 \\
\hline 15 & 1 & -3.081103 & -0.908303 & -0.684322 \\
\hline 16 & 1 & -1.811083 & -1.579645 & 0.399845 \\
\hline 17 & 1 & -2.981105 & -0.371904 & 0.986912 \\
\hline
\end{tabular}

Energy: $\mathrm{HF}=-234.9055725$

Zero-Point Energy Correction: $\mathbf{0 . 1 5 0 3 8 3}$

Imaginary Frequency: One imaginary frequency $-421.4160 \mathrm{~cm}^{-1}$ 
b3lyp/6-31G(d)

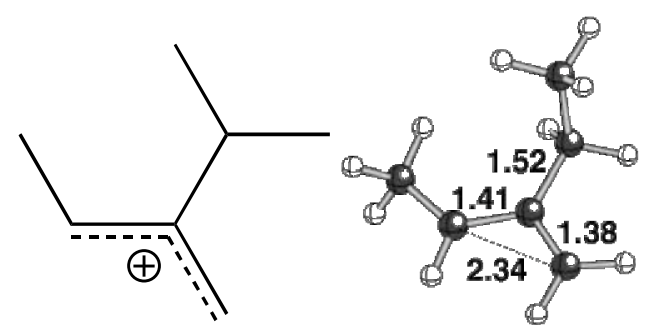

\section{Coordinates:}

Standard orientation:

\begin{tabular}{|c|c|c|c|c|}
\hline \multirow{2}{*}{$\begin{array}{l}\text { Center } \\
\text { Number }\end{array}$} & Atomic & \multirow{2}{*}{$\begin{array}{c}\text { Atomic } \\
\text { Type }\end{array}$} & \multicolumn{2}{|c|}{ Coordinates (Angstroms) } \\
\hline & Number & & X $\quad Y$ & I $\quad \mathrm{Z}$ \\
\hline 1 & 6 & -1.306568 & 0.289099 & -0.143721 \\
\hline 2 & 6 & 0.027298 & 0.657343 & 0.132439 \\
\hline 3 & 6 & 0.312812 & 1.980390 & -0.126180 \\
\hline 4 & 6 & 1.070775 & -0.310736 & 0.650735 \\
\hline 5 & 1 & 0.617958 & -0.971704 & 1.397646 \\
\hline 6 & 6 & -1.921569 & -1.023444 & 0.039504 \\
\hline 7 & 1 & -2.789604 & -0.911987 & 0.713749 \\
\hline 8 & 1 & -2.380444 & -1.336467 & -0.914437 \\
\hline 9 & 1 & -1.254570 & -1.800300 & 0.410747 \\
\hline 10 & 1 & -1.964347 & 1.070828 & -0.526997 \\
\hline 11 & 1 & -0.435013 & 2.665618 & -0.519095 \\
\hline 12 & 1 & 1.842674 & 0.259745 & 1.177212 \\
\hline 13 & 1 & 1.302245 & 2.395074 & 0.056370 \\
\hline 14 & 6 & 1.716912 & -1.142692 & -0.474441 \\
\hline 15 & 1 & 2.460413 & -1.821921 & -0.048888 \\
\hline 16 & 1 & 0.979797 & -1.747965 & -1.013506 \\
\hline 17 & 1 & 2.222928 & -0.500682 & -1.202823 \\
\hline
\end{tabular}

Energy: $\mathrm{HF}=-234.9545559$

Zero-Point Energy Correction: $\mathbf{0 . 1 5 3 8 0 0}$

Imaginary Frequency: Minimum (No imaginary frequencies) 


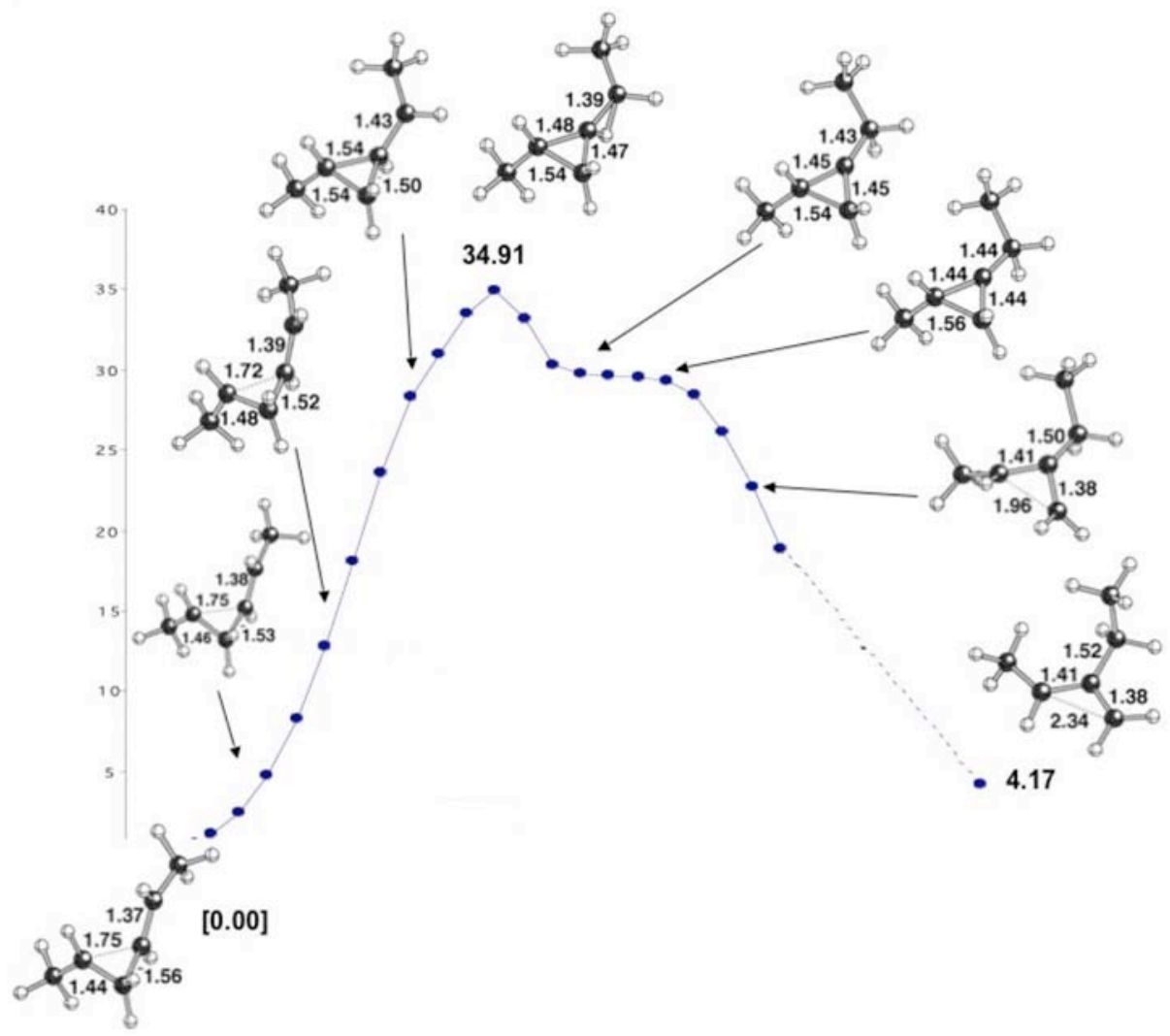




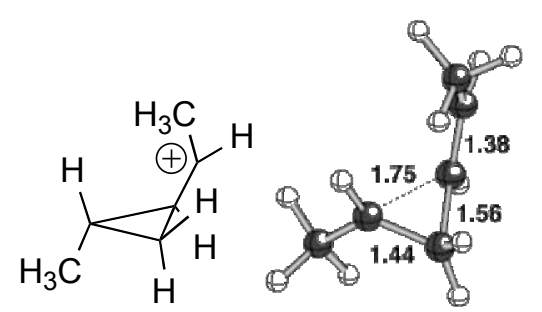

b3lyp/6-31G(d)

\section{Coordinates:}

Standard orientation:

\begin{tabular}{|c|c|c|c|c|c|}
\hline \multirow{2}{*}{$\begin{array}{l}\text { Center } \\
\text { Number }\end{array}$} & \multirow{2}{*}{\multicolumn{2}{|c|}{$\begin{array}{l}\text { Atomic } \\
\text { Number }\end{array}$}} & \multirow{2}{*}{$\begin{array}{l}\text { Atomic } \\
\text { Type }\end{array}$} & \multicolumn{2}{|c|}{ Coordinates (Angstroms) } \\
\hline & & & & X $\quad Y$ & $\mathrm{Z}$ \\
\hline 1 & 6 & 0 & -0.955838 & -0.063939 & -0.510105 \\
\hline 2 & 6 & 0 & 0.136889 & 0.224077 & 0.827411 \\
\hline 3 & 6 & 0 & -0.516062 & 1.258077 & -0.142531 \\
\hline 4 & 6 & 0 & 1.381570 & -0.328265 & 0.640331 \\
\hline 5 & 1 & 0 & -0.360314 & 0.086844 & 1.781020 \\
\hline 6 & 6 & 0 & -2.255816 & -0.673069 & -0.092116 \\
\hline 7 & 1 & 0 & -2.946578 & -0.556482 & -0.940808 \\
\hline 8 & 1 & 0 & -2.165271 & -1.745172 & 0.104194 \\
\hline 9 & 1 & 0 & -2.699801 & -0.171629 & 0.771769 \\
\hline 10 & 1 & 0 & -0.423870 & -0.561791 & -1.316924 \\
\hline 11 & 1 & 0 & 0.186176 & 1.779916 & -0.786475 \\
\hline 12 & 1 & 0 & -1.213052 & 1.902393 & 0.389145 \\
\hline 13 & 1 & 0 & 1.791936 & -0.879755 & 1.487897 \\
\hline 14 & 6 & 0 & 2.252738 & -0.262029 & -0.551425 \\
\hline 15 & 1 & 0 & 3.201587 & 0.210979 & -0.253835 \\
\hline 16 & 1 & 0 & 2.530957 & -1.278285 & -0.865104 \\
\hline 17 & 1 & 0 & 1.837344 & 0.283865 & -1.400271 \\
\hline
\end{tabular}

Energy: $\mathrm{HF}=-234.9582934$

Zero-Point Energy Correction: 0.154486 Imaginary Frequency: Minimum (No imaginary frequencies) 


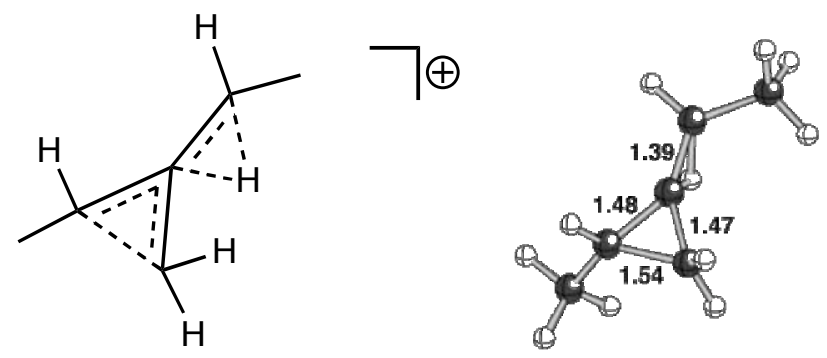

b3lyp/6-31G(d)

Coordinates:

Standard orientation:

\begin{tabular}{|c|c|c|c|c|}
\hline \multirow{2}{*}{$\begin{array}{l}\text { Center } \\
\text { Number }\end{array}$} & \multirow{2}{*}{$\begin{array}{l}\text { Atomic } \\
\text { Number }\end{array}$} & \multirow{2}{*}{$\begin{array}{r}\text { Atomic } \\
\text { Type }\end{array}$} & \multicolumn{2}{|c|}{ Coordinates (Angstroms } \\
\hline & & & X $\quad Y$ & $\mathrm{Z}$ \\
\hline 1 & 6 & 1.284429 & 0.067978 & 0.498448 \\
\hline 2 & 6 & -0.135648 & 0.014438 & 0.097002 \\
\hline 3 & 6 & 0.561624 & 1.297322 & -0.086358 \\
\hline 4 & 6 & -1.358643 & -0.631709 & 0.182991 \\
\hline 5 & 1 & -0.498562 & -0.694624 & -0.895698 \\
\hline 6 & 6 & 2.386394 & -0.617298 & -0.292469 \\
\hline 7 & 1 & 3.338516 & -0.115286 & -0.099530 \\
\hline 8 & 1 & 2.485708 & -1.664789 & 0.007887 \\
\hline 9 & 1 & 2.194708 & -0.579570 & -1.370401 \\
\hline 10 & 1 & 1.419279 & 0.055143 & 1.581026 \\
\hline 11 & 1 & 0.258958 & 2.121409 & 0.557158 \\
\hline 12 & 1 & 0.854354 & 1.592341 & -1.092361 \\
\hline 13 & 1 & -1.345954 & -1.660788 & 0.553043 \\
\hline 14 & 6 & -2.676328 & -0.029015 & $5-0.166379$ \\
\hline 15 & 1 & -3.272761 & -0.699004 & $4-0.793388$ \\
\hline 16 & 1 & -3.223672 & 0.079951 & 0.782512 \\
\hline 17 & 1 & -2.581540 & 0.954930 & -0.629656 \\
\hline
\end{tabular}

Energy: $H F=-234.9058394$

Zero-Point Energy Correction: 0.150329

Imaginary Frequency: One imaginary frequency $-433.2766 \mathrm{~cm}^{-1}$ 

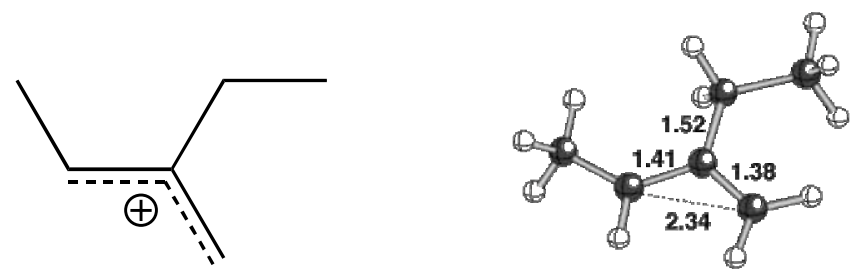

b31yp/6-31G(d)

Coordinates:

Standard orientation:

\begin{tabular}{|c|c|c|c|c|}
\hline \multirow{2}{*}{$\begin{array}{l}\text { Center } \\
\text { Number }\end{array}$} & \multirow{2}{*}{$\begin{array}{l}\text { Atomic } \\
\text { Number }\end{array}$} & \multirow{2}{*}{$\begin{array}{r}\text { Atomic } \\
\text { Type }\end{array}$} & \multicolumn{2}{|c|}{ Coordinates (Angstro1 } \\
\hline & & & $\mathrm{X} \quad \mathrm{Y}$ & l $\quad \mathrm{Z}$ \\
\hline 1 & 6 & 1.439317 & 0.417233 & -0.000046 \\
\hline 2 & 6 & 52 & 0.437981 & -0.000038 \\
\hline 3 & 6 & -0.514641 & 1.704154 & 0.000016 \\
\hline 4 & 6 & -0.7 & -0.837477 & -0.000049 \\
\hline 5 & 1 & -0.5 & -1.430991 & -0.876017 \\
\hline 6 & 6 & & -0.761320 & 0.000040 \\
\hline 7 & 1 & .985493 & -0.698142 & -0.865536 \\
\hline 8 & 1 & 29 & -0.698163 & 0.865827 \\
\hline 9 & 1 & 1.78 & -1.717246 & -0.000042 \\
\hline 10 & 1 & 0 & 1.381459 & -0.000065 \\
\hline 11 & 1 & & 2.592 & 0.000022 \\
\hline 12 & 1 & -1.588373 & 1.869192 & 0.000105 \\
\hline 13 & 1 & -0.501015 & -1.431088 & 0.875819 \\
\hline 14 & 6 & -2.312149 & -0.637302 & 0.000032 \\
\hline 15 & 1 & -2.806 & -1.611741 & 0.000105 \\
\hline 16 & 1 & -2.654 & -0.097645 & 0.890348 \\
\hline 17 & 1 & -2.654261 & -0.097732 & -0.890293 \\
\hline
\end{tabular}

Energy: $H F=-234.9550667$

Zero-Point Energy Correction: $\mathbf{0 . 1 5 3 8 0 4}$

Imaginary Frequency: Minimum (No imaginary frequencies) 


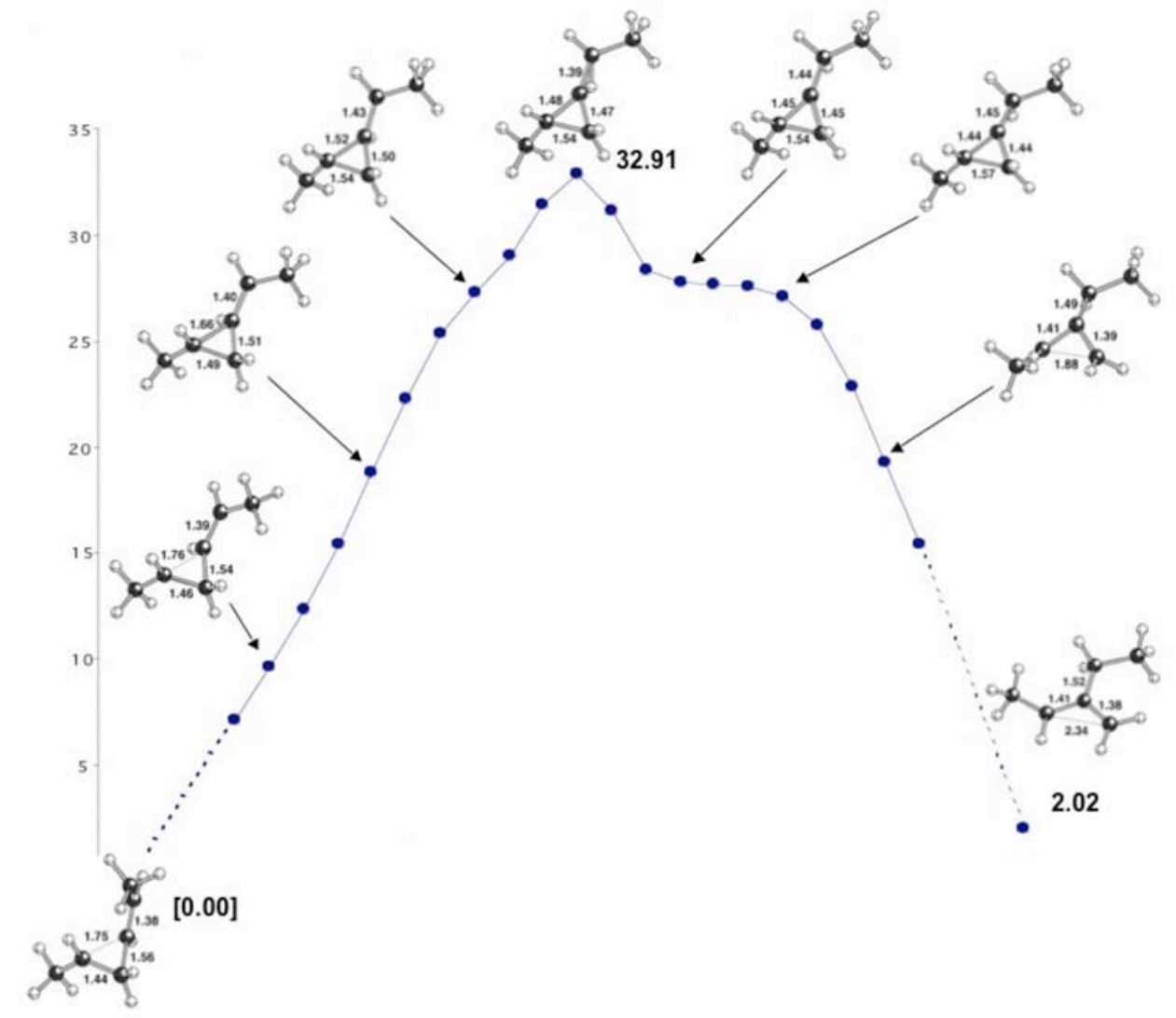




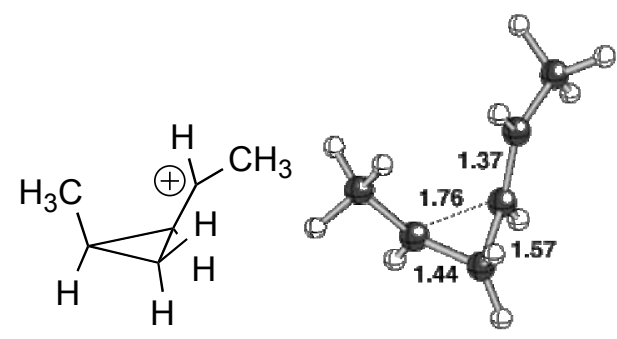

b3lyp/6-31G(d)

\section{Coordinates:}

Standard orientation:

\begin{tabular}{|c|c|c|c|c|c|}
\hline \multirow{2}{*}{$\begin{array}{l}\text { Center } \\
\text { Number }\end{array}$} & \multirow{2}{*}{\multicolumn{2}{|c|}{$\begin{array}{l}\text { Atomic } \\
\text { Number }\end{array}$}} & \multirow{2}{*}{$\begin{array}{r}\text { Atomic } \\
\text { Type }\end{array}$} & \multicolumn{2}{|c|}{ Coordinates (Angstroms) } \\
\hline & & & & X $\quad Y$ & $\mathrm{Z}$ \\
\hline 1 & 6 & 0 & -1.351787 & -0.111698 & -0.436416 \\
\hline 2 & 6 & 0 & 0.290405 & -0.730872 & -0.277324 \\
\hline 3 & 6 & 0 & -1.029950 & -1.221128 & 0.417869 \\
\hline 4 & 6 & 0 & 1.165131 & 0.128540 & 0.331835 \\
\hline 5 & 1 & 0 & 0.601236 & -1.286492 & -1.155927 \\
\hline 6 & 1 & 0 & -1.058901 & -1.068888 & 1.494098 \\
\hline 7 & 1 & 0 & -1.270071 & -2.231601 & 0.094357 \\
\hline 8 & 6 & 0 & 2.558348 & 0.364785 & -0.093906 \\
\hline 9 & 1 & 0 & 3.233348 & 0.115686 & 0.739607 \\
\hline 10 & 1 & 0 & 2.845607 & -0.207009 & -0.978291 \\
\hline 11 & 1 & 0 & 2.714082 & 1.438915 & -0.274148 \\
\hline 12 & 6 & 0 & -1.740803 & 1.253439 & 0.040707 \\
\hline 13 & 1 & 0 & -1.367853 & 2.046526 & -0.612815 \\
\hline 14 & 1 & 0 & -2.839024 & 1.288780 & -0.002784 \\
\hline 15 & 1 & 0 & -1.450614 & 1.449549 & 1.075689 \\
\hline 16 & 1 & 0 & -1.577922 & -0.333522 & -1.478189 \\
\hline 17 & 1 & 0 & 0.822040 & 0.689658 & 1.201811 \\
\hline
\end{tabular}

Energy: $\mathrm{HF}=-234.9579374$

Zero-Point Energy Correction: 0.154215

Imaginary Frequency: Minimum (No imaginary frequencies) 
<smiles>CC1CC2C=CC1C2C</smiles>

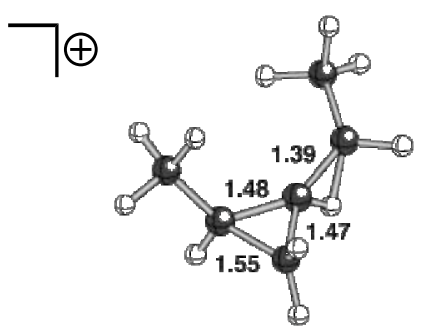

b3lyp/6-31G(d)

\section{Coordinates:}

Standard orientation:

\begin{tabular}{|c|c|c|c|c|c|}
\hline \multirow{2}{*}{$\begin{array}{l}\text { Center } \\
\text { Number }\end{array}$} & \multirow{2}{*}{\multicolumn{2}{|c|}{$\begin{array}{l}\text { Atomic } \\
\text { Number }\end{array}$}} & \multirow{2}{*}{$\begin{array}{l}\text { Atomic } \\
\text { Type }\end{array}$} & \multicolumn{2}{|c|}{ Coordinates (Angstrom } \\
\hline & & & & X $\quad Y$ & $\mathrm{Z}$ \\
\hline 1 & 6 & 0 & 1.161729 & 0.091036 & 0.489007 \\
\hline 2 & 6 & 0 & -0.073372 & -0.613937 & 0.095690 \\
\hline 3 & 6 & 0 & 1.198270 & -1.297578 & -0.187553 \\
\hline 4 & 6 & 0 & -1.418542 & -0.518962 & -0.226561 \\
\hline 5 & 1 & 0 & -0.906169 & -1.089797 & 0.926093 \\
\hline 6 & 1 & 0 & 1.475346 & -1.385168 & -1.237285 \\
\hline 7 & 1 & 0 & 1.502326 & -2.136504 & 0.433529 \\
\hline 8 & 6 & 0 & -2.295629 & 0.650759 & 0.064085 \\
\hline 9 & 1 & 0 & -3.245023 & 0.339820 & 0.511232 \\
\hline 10 & 1 & 0 & -1.805787 & 1.405496 & 0.681853 \\
\hline 11 & 1 & 0 & -2.542707 & 1.098511 & -0.910660 \\
\hline 12 & 6 & 0 & 1.575923 & 1.361846 & -0.231069 \\
\hline 13 & 1 & 0 & 1.128031 & 2.244386 & 0.235721 \\
\hline 14 & 1 & 0 & 2.663958 & 1.465893 & -0.178156 \\
\hline 15 & 1 & 0 & 1.287605 & 1.340868 & -1.286955 \\
\hline 16 & 1 & 0 & 1.412515 & 0.051870 & 1.548782 \\
\hline 17 & 1 & 0 & -1.860362 & -1.374361 & -0.745760 \\
\hline
\end{tabular}

Energy: $\mathrm{HF}=-234.905864$

Zero-Point Energy Correction: 0.150213

Imaginary Frequency: One imaginary frequency $-446.975 \mathrm{~cm}^{-1}$ 

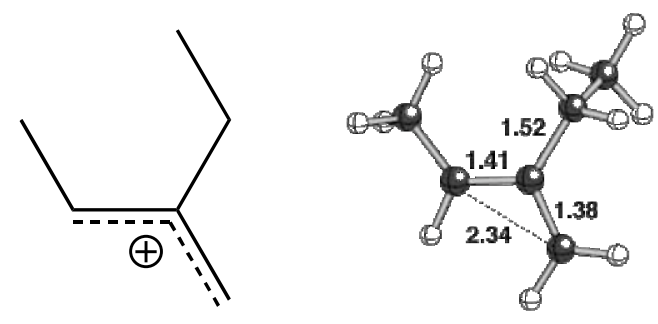

b3lyp/6-31G(d)

\section{Coordinates:}

Standard orientation:

\begin{tabular}{|c|c|c|c|c|c|}
\hline \multirow{2}{*}{$\begin{array}{l}\text { Center } \\
\text { Number }\end{array}$} & \multirow{2}{*}{$\begin{array}{l}\text { Atomic } \\
\text { Number }\end{array}$} & \multirow{2}{*}{\multicolumn{2}{|c|}{$\begin{array}{l}\text { Atomic } \\
\text { Type }\end{array}$}} & \multicolumn{2}{|c|}{ Coordinates (Angstron } \\
\hline & & & & X $\quad Y$ & $Y \quad Z$ \\
\hline 1 & 6 & 0 & 1.306498 & 0.289370 & -0.143501 \\
\hline 2 & 6 & 0 & -0.027406 & 0.657454 & 0.132336 \\
\hline 3 & 6 & 0 & -0.313193 & 1.980528 & -0.126481 \\
\hline 4 & 6 & 0 & -1.070957 & -0.310461 & 0.650665 \\
\hline 5 & 1 & 0 & -1.843420 & 0.260154 & 1.176159 \\
\hline 6 & 1 & 0 & -1.302596 & 2.395164 & 0.056326 \\
\hline 7 & 1 & 0 & 0.434666 & 2.665931 & -0.519017 \\
\hline 8 & 6 & 0 & -1.716047 & -1.143450 & -0.474400 \\
\hline 9 & 1 & 0 & -2.460420 & -1.821786 & -0.048962 \\
\hline 10 & 1 & 0 & -2.220780 & -0.502069 & $\begin{array}{l}9 \\
-1.204219\end{array}$ \\
\hline 11 & 1 & 0 & -0.978509 & -1.749721 & $1-1.011744$ \\
\hline 12 & 6 & 0 & 1.921401 & -1.023340 & 0.039677 \\
\hline 13 & 1 & 0 & 2.379713 & -1.336130 & -0.914639 \\
\hline 14 & 1 & 0 & 2.789571 & -0.912479 & 0.713714 \\
\hline 15 & 1 & 0 & 1.254132 & -1.799987 & 0.410854 \\
\hline 16 & 1 & 0 & 1.964348 & 1.071106 & -0.526596 \\
\hline 17 & 1 & 0 & -0.618476 & -0.970793 & $3 \quad 1.398347$ \\
\hline
\end{tabular}

Energy: $\mathrm{HF}=-234.9545559$

Zero-Point Energy Correction: 0.153799

Imaginary Frequency: Minimum (No imaginary frequencies) 


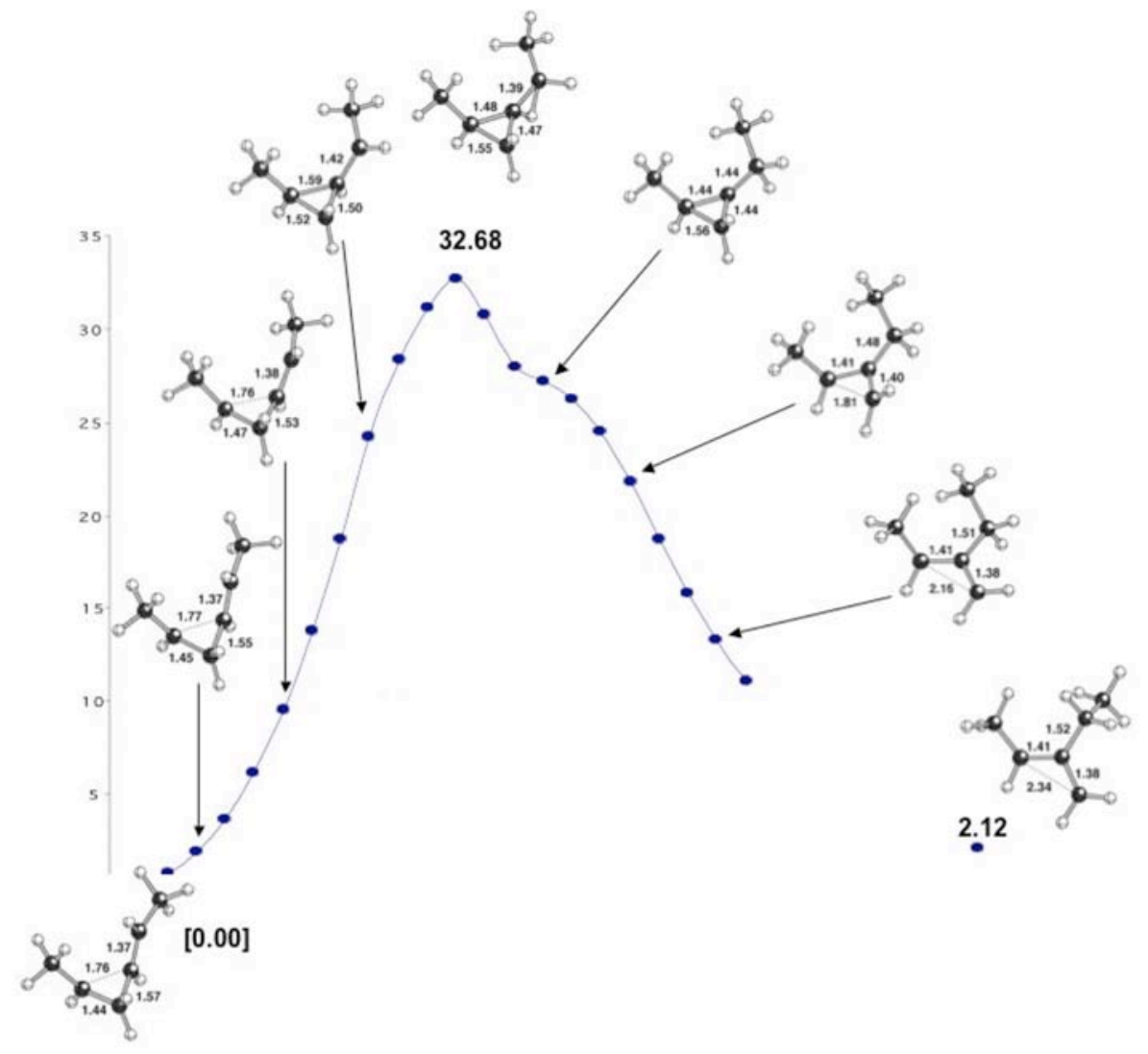




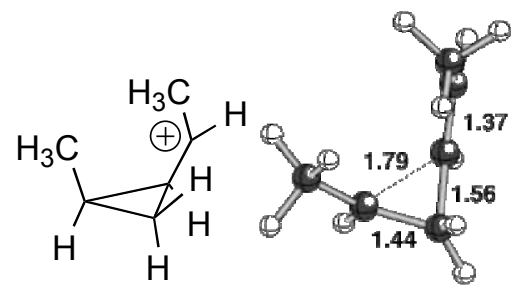

b3lyp/6-31G(d)

\section{Coordinates:}

Standard orientation:

\begin{tabular}{|c|c|c|c|c|c|}
\hline \multirow{2}{*}{$\begin{array}{l}\text { Center } \\
\text { Number }\end{array}$} & \multirow{2}{*}{\multicolumn{2}{|c|}{$\begin{array}{l}\text { Atomic } \\
\text { Number }\end{array}$}} & \multirow{2}{*}{$\begin{array}{l}\text { Atomic } \\
\text { Type }\end{array}$} & \multicolumn{2}{|c|}{ Coordinates (Angstroms } \\
\hline & & & & $\begin{array}{ll}X & Y\end{array}$ & $\mathrm{Z}$ \\
\hline 1 & 6 & 0 & -1.327903 & -0.133524 & -0.209440 \\
\hline 2 & 6 & 0 & 0.189299 & -1.055777 & -0.397025 \\
\hline 3 & 6 & 0 & -0.794736 & -0.998594 & 0.811572 \\
\hline 4 & 6 & 0 & 1.362525 & -0.348979 & -0.492051 \\
\hline 5 & 1 & 0 & 0.020133 & -1.860722 & -1.103508 \\
\hline 6 & 1 & 0 & -0.390325 & -0.538968 & 1.710033 \\
\hline 7 & 1 & 0 & -1.259751 & -1.969455 & 0.971439 \\
\hline 8 & 6 & 0 & -1.370356 & 1.357559 & -0.168843 \\
\hline 9 & 1 & 0 & -1.198800 & 1.806081 & -1.151472 \\
\hline 10 & 1 & 0 & -2.400870 & 1.615186 & 0.120591 \\
\hline 11 & 1 & 0 & -0.703591 & 1.800451 & 0.572216 \\
\hline 12 & 1 & 0 & -1.953496 & -0.597274 & -0.970589 \\
\hline 13 & 6 & 0 & 1.885484 & 0.733255 & 0.366454 \\
\hline 14 & 1 & 0 & 2.003803 & 1.650510 & -0.231291 \\
\hline 15 & 1 & 0 & 1.290330 & 0.950176 & 1.253357 \\
\hline 16 & 1 & 0 & 2.906163 & 0.465197 & 0.676133 \\
\hline 17 & 1 & 0 & 2.020528 & -0.644823 & -1.310914 \\
\hline
\end{tabular}

Energy: $H F=-234.9536636$

Zero-Point Energy Correction: 0.154316

Imaginary Frequency: Minimum (No imaginary frequencies) 
<smiles>CC1=CC=CC1C</smiles>

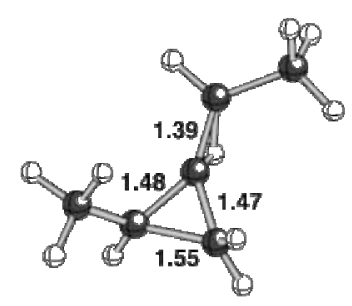

b3lyp/6-31G(d)

Coordinates:

Standard orientation:

\begin{tabular}{|c|c|c|c|c|c|}
\hline \multirow{2}{*}{$\begin{array}{l}\text { Center } \\
\text { Number }\end{array}$} & \multirow{2}{*}{$\begin{array}{l}\text { Atomic } \\
\text { Number }\end{array}$} & \multirow{2}{*}{\multicolumn{2}{|c|}{$\begin{array}{l}\text { Atomic } \\
\text { Type }\end{array}$}} & \multicolumn{2}{|c|}{ Coordinates (Angstroms } \\
\hline & & & & X $\quad Y$ & Z $\quad \mathrm{Z}$ \\
\hline 1 & 6 & 0 & 1.353130 & 0.166923 & -0.425920 \\
\hline 2 & 6 & 0 & -0.118371 & 0.163969 & -0.313060 \\
\hline 3 & 6 & 0 & 0.583805 & 1.332054 & 0.237248 \\
\hline 4 & 6 & ) & -1.287236 & -0.581044 & -0.291311 \\
\hline 5 & 1 & 0 & -0.877226 & 0.225606 & -1.332424 \\
\hline 6 & 1 & 0 & 0.578218 & 1.434983 & 1.321692 \\
\hline 7 & 1 & 0 & 0.562165 & 2.272914 & -0.307558 \\
\hline 8 & 6 & 0 & 2.174131 & -0.833849 & 0.366631 \\
\hline 9 & 1 & 0 & 2.287657 & -1.772162 & -0.184727 \\
\hline 10 & 1 & 0 & 3.171724 & -0.420412 & 0.543758 \\
\hline 11 & 1 & 0 & 1.717924 & -1.052592 & 1.337438 \\
\hline 12 & 1 & 0 & 1.753423 & 0.427356 & -1.405223 \\
\hline 13 & 6 & 0 & -2.577624 & -0.138723 & 0.310344 \\
\hline 14 & 1 & 0 & -2.736681 & -0.772931 & 1.195895 \\
\hline 15 & 1 & 0 & -2.559639 & 0.905666 & 0.627861 \\
\hline 16 & 1 & 0 & -3.420789 & -0.326451 & -0.361580 \\
\hline 17 & 1 & 0 & -1.243790 & -1.577959 & -0.738728 \\
\hline
\end{tabular}

Energy: $\mathrm{HF}=-234.9062794$

Zero-Point Energy Correction: 0.150268

Imaginary Frequency: One imaginary frequency $-454.2682 \mathrm{~cm}^{-1}$ 

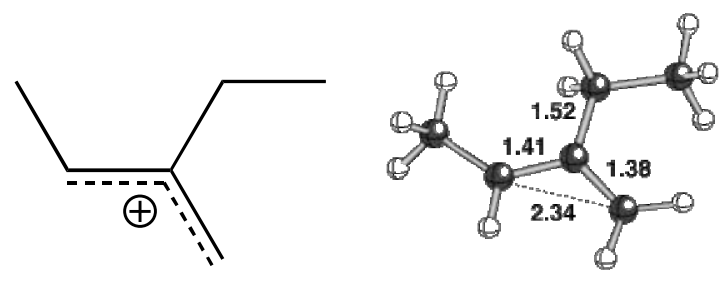

b3lyp/6-31G(d)

\section{Coordinates:}

Standard orientation:

\begin{tabular}{|c|c|c|c|c|c|}
\hline \multirow{2}{*}{$\begin{array}{l}\text { Center } \\
\text { Number }\end{array}$} & \multirow{2}{*}{$\begin{array}{l}\text { Atomic } \\
\text { Number }\end{array}$} & \multirow{2}{*}{\multicolumn{2}{|c|}{$\begin{array}{r}\text { Atomic } \\
\text { Type }\end{array}$}} & \multicolumn{2}{|c|}{ Coordinates (Angstro } \\
\hline & & & & $\begin{array}{ll}\mathrm{X} & \mathrm{Y}\end{array}$ & $Z$ \\
\hline 1 & 6 & 0 & -1.439328 & 0.417258 & -0.000075 \\
\hline 2 & 6 & 0 & -0.028564 & 0.437981 & -0.000061 \\
\hline 3 & 6 & 0 & 0.514683 & 1.704137 & 0.000004 \\
\hline 4 & 6 & 0 & 0.794841 & -0.837488 & -0.000117 \\
\hline 5 & 1 & 0 & 0.500937 & -1.431160 & 0.875686 \\
\hline 6 & 1 & 0 & 1.588419 & 1.869148 & 0.000038 \\
\hline 7 & 1 & 0 & -0.112282 & 2.592485 & 0.000062 \\
\hline 8 & 6 & 0 & -2.302502 & -0.761297 & -0.000005 \\
\hline 9 & 1 & 0 & -2.984085 & -0.698802 & 0.866777 \\
\hline 10 & 1 & 0 & -2.986580 & -0.697571 & -0.86465 \\
\hline 11 & 1 & 0 & -1.780562 & -1.717213 & -0.00124 \\
\hline 12 & 1 & 0 & -1.949817 & 1.381499 & 0.00002 \\
\hline 13 & 6 & 0 & 2.312138 & -0.637310 & 0.00009 \\
\hline 14 & 1 & 0 & 2.654308 & -0.097642 & -0.89015 \\
\hline 15 & 1 & 0 & 2.654075 & -0.097753 & 0.89049 \\
\hline 16 & 1 & 0 & 2.806813 & -1.611749 & 0.00009 \\
\hline 17 & 1 & 0 & 0.501160 & -1.430932 & -0.87615 \\
\hline
\end{tabular}

Energy: $\mathrm{HF}=-234.9550667$

Zero-Point Energy Correction: 0.153804

Imaginary Frequency: Minimum (No imaginary frequencies) 


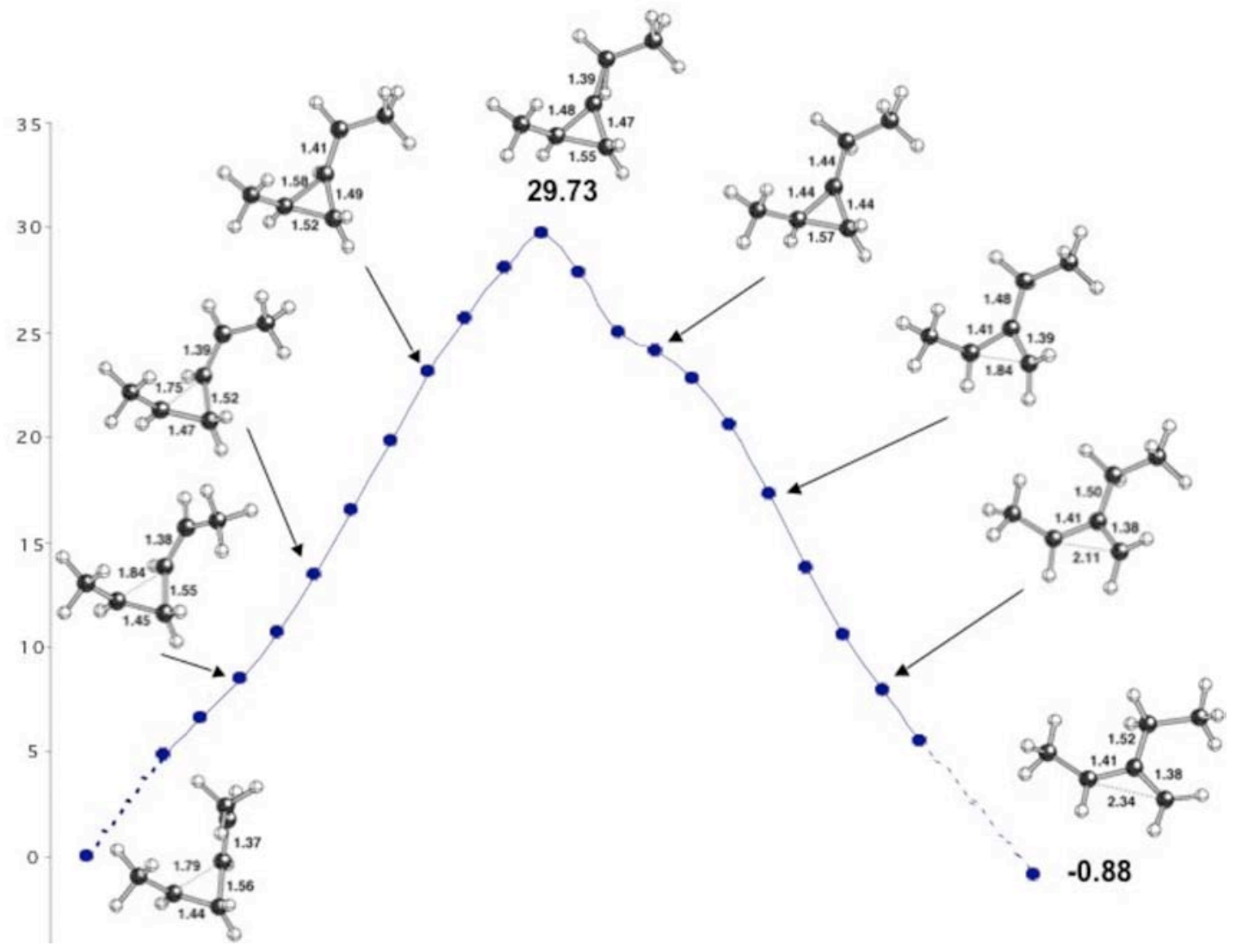


<smiles>C[CH][C@H]1C[C@H](C)C1C</smiles>

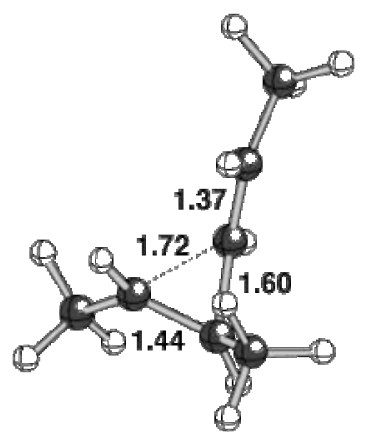

b3lyp/6-31G(d)

\section{Coordinates:}

Standard orientation:

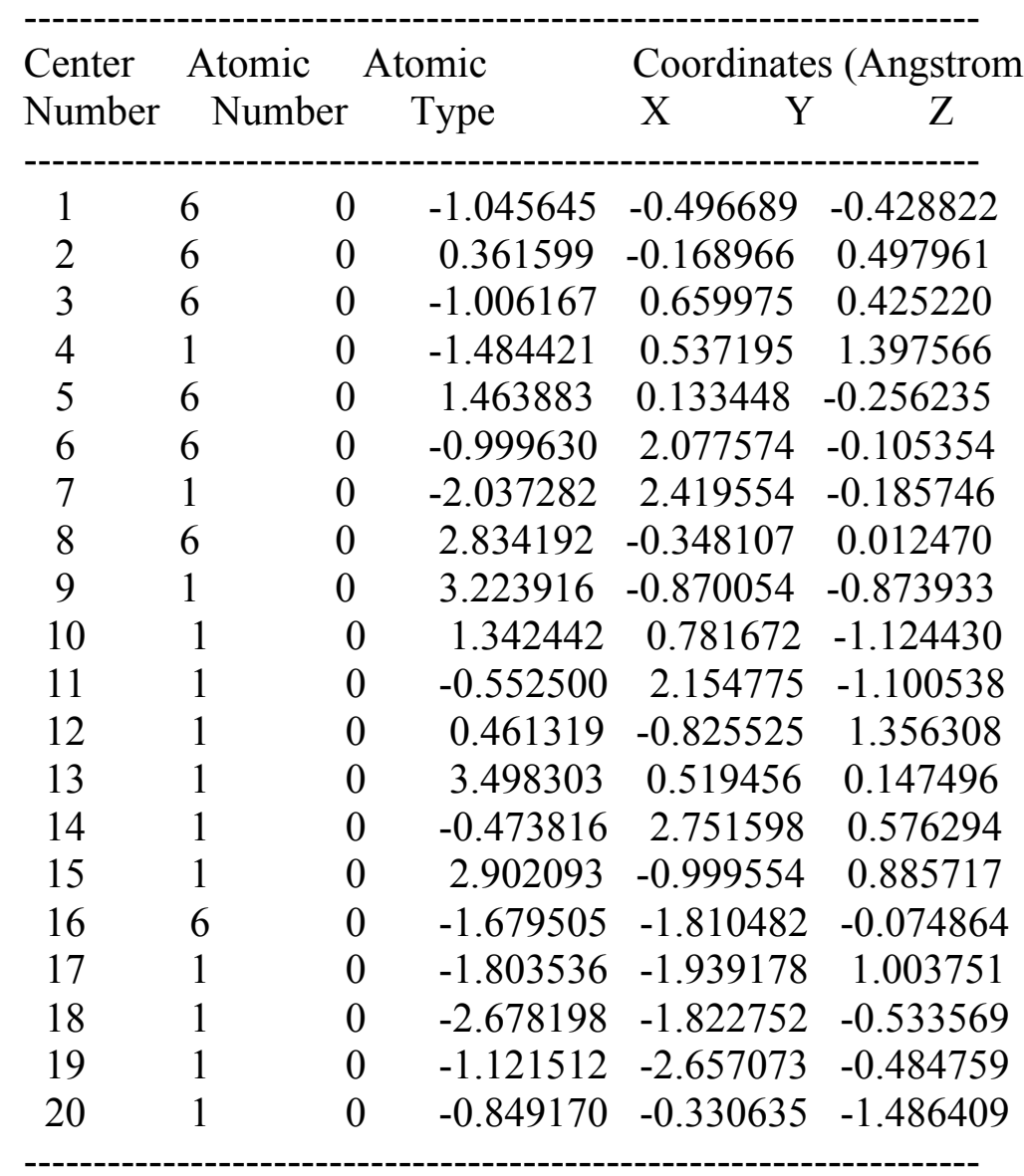

Energy: $H F=-274.2813266$ 

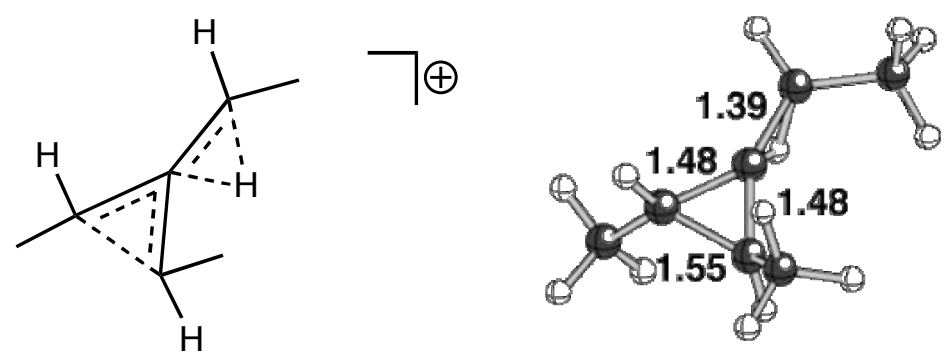

b3lyp/6-31G(d)

\section{Coordinates:}

Standard orientation:

\begin{tabular}{|c|c|c|c|c|}
\hline \multirow{2}{*}{$\begin{array}{l}\text { Center } \\
\text { Number }\end{array}$} & \multirow{2}{*}{$\begin{array}{l}\text { Atomic } \\
\text { Number }\end{array}$} & \multirow{2}{*}{$\begin{array}{r}\text { Atomic } \\
\text { Type }\end{array}$} & \multicolumn{2}{|c|}{ Coordinates (Angstron } \\
\hline & & & $\mathrm{X}$ & $\mathrm{Z}$ \\
\hline 1 & 6 & -1.249742 & -0.282207 & -0.431014 \\
\hline 2 & 6 & 0.169632 & -0.363938 & -0.027922 \\
\hline 3 & 6 & -0.582095 & 0.828530 & 0.413056 \\
\hline 4 & 1 & -0.909975 & 0.815427 & 1.453228 \\
\hline 5 & 6 & 1.413195 & -0.920652 & -0.287458 \\
\hline 6 & 6 & -0.239744 & 2.200239 & -0.140755 \\
\hline 7 & 1 & -1.126477 & 2.840027 & -0.095480 \\
\hline 8 & 6 & 2.719169 & -0.373525 & 0.177111 \\
\hline 9 & 1 & 3.347435 & -1.152732 & 0.620125 \\
\hline 10 & 1 & 1.428156 & -1.836059 & -0.886298 \\
\hline 11 & 1 & 0.085868 & 2.145281 & -1.184609 \\
\hline 12 & 1 & 0.566629 & -1.257676 & 0.770240 \\
\hline 13 & 1 & 3.245141 & -0.026477 & -0.725553 \\
\hline 14 & 1 & 0.551508 & 2.678837 & 0.444747 \\
\hline 15 & 1 & 2.605979 & 0.468931 & 0.861685 \\
\hline 16 & 6 & -2.328780 & -1.163986 & 0.174530 \\
\hline 17 & 1 & -2.152236 & -1.345813 & 1.240334 \\
\hline 18 & 1 & -3.302007 & -0.675923 & 0.070470 \\
\hline 19 & 1 & -2.376324 & -2.129516 & -0.338871 \\
\hline 20 & 1 & -1.373510 & -0.071067 & -1.495301 \\
\hline
\end{tabular}

Energy: HF $=-274.2259835$

Zero-Point Energy Correction: $\mathbf{0 . 1 7 8 4 8 4}$

Imaginary Frequency: One imaginary frequency $-459.1279 \mathrm{~cm}^{-1}$ 

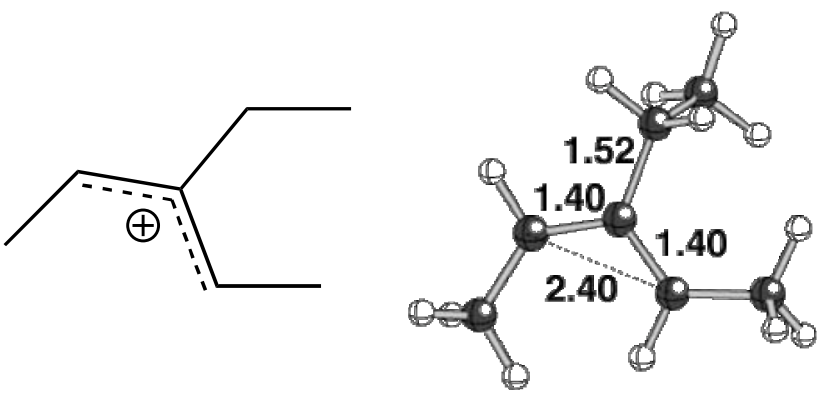

b3lyp/6-31G(d)

Coordinates:

Standard orientation:

\begin{tabular}{|c|c|c|c|c|c|}
\hline \multirow{2}{*}{$\begin{array}{l}\text { Center } \\
\text { Number }\end{array}$} & \multirow{2}{*}{\multicolumn{2}{|c|}{$\begin{array}{l}\text { Atomic } \\
\text { Number }\end{array}$}} & \multirow{2}{*}{$\begin{array}{l}\text { Atomic } \\
\text { Type }\end{array}$} & \multicolumn{2}{|c|}{ Coordinates (Angstroms) } \\
\hline & & & & X $\quad Y$ & $\mathrm{Z}$ \\
\hline 1 & 6 & 0 & -1.405649 & -0.688207 & -0.152760 \\
\hline 2 & 6 & 0 & -0.141790 & -0.095012 & -0.246754 \\
\hline 3 & 6 & 0 & -0.030390 & 1.263464 & 0.049734 \\
\hline 4 & 1 & 0 & -0.935242 & 1.800257 & 0.327651 \\
\hline 5 & 6 & 0 & 1.052713 & -0.949443 & -0.639062 \\
\hline 6 & 6 & 0 & 1.188999 & 2.082281 & 0.021612 \\
\hline 7 & 1 & 0 & 1.036449 & 2.920025 & -0.679521 \\
\hline 8 & 6 & 0 & 1.833389 & -1.480824 & 0.577233 \\
\hline 9 & 1 & 0 & 2.676433 & -2.090720 & 0.241002 \\
\hline 10 & 1 & 0 & 1.717638 & -0.374418 & -1.291528 \\
\hline 11 & 1 & 0 & 2.099149 & 1.543923 & -0.238827 \\
\hline 12 & 1 & 0 & 0.696694 & -1.791266 & -1.242976 \\
\hline 13 & 1 & 0 & 2.231735 & -0.667059 & 1.193128 \\
\hline 14 & 1 & 0 & 1.311388 & 2.573984 & 1.000465 \\
\hline 15 & 1 & 0 & 1.199959 & -2.105216 & 1.216628 \\
\hline 16 & 6 & 0 & -2.716829 & -0.126012 & 0.215183 \\
\hline 17 & 1 & 0 & -2.738966 & 0.934081 & 0.466056 \\
\hline 18 & 1 & 0 & -3.421871 & -0.320322 & -0.608651 \\
\hline 19 & 1 & 0 & -3.118609 & -0.708015 & 1.059909 \\
\hline 20 & 1 & 0 & -1.437411 & -1.752745 & -0.394448 \\
\hline
\end{tabular}

Energy: $\mathrm{HF}=-274.2858628$

Zero-Point Energy Correction: 0.181971

Imaginary Frequency: Minimum (No imaginary frequencies) 


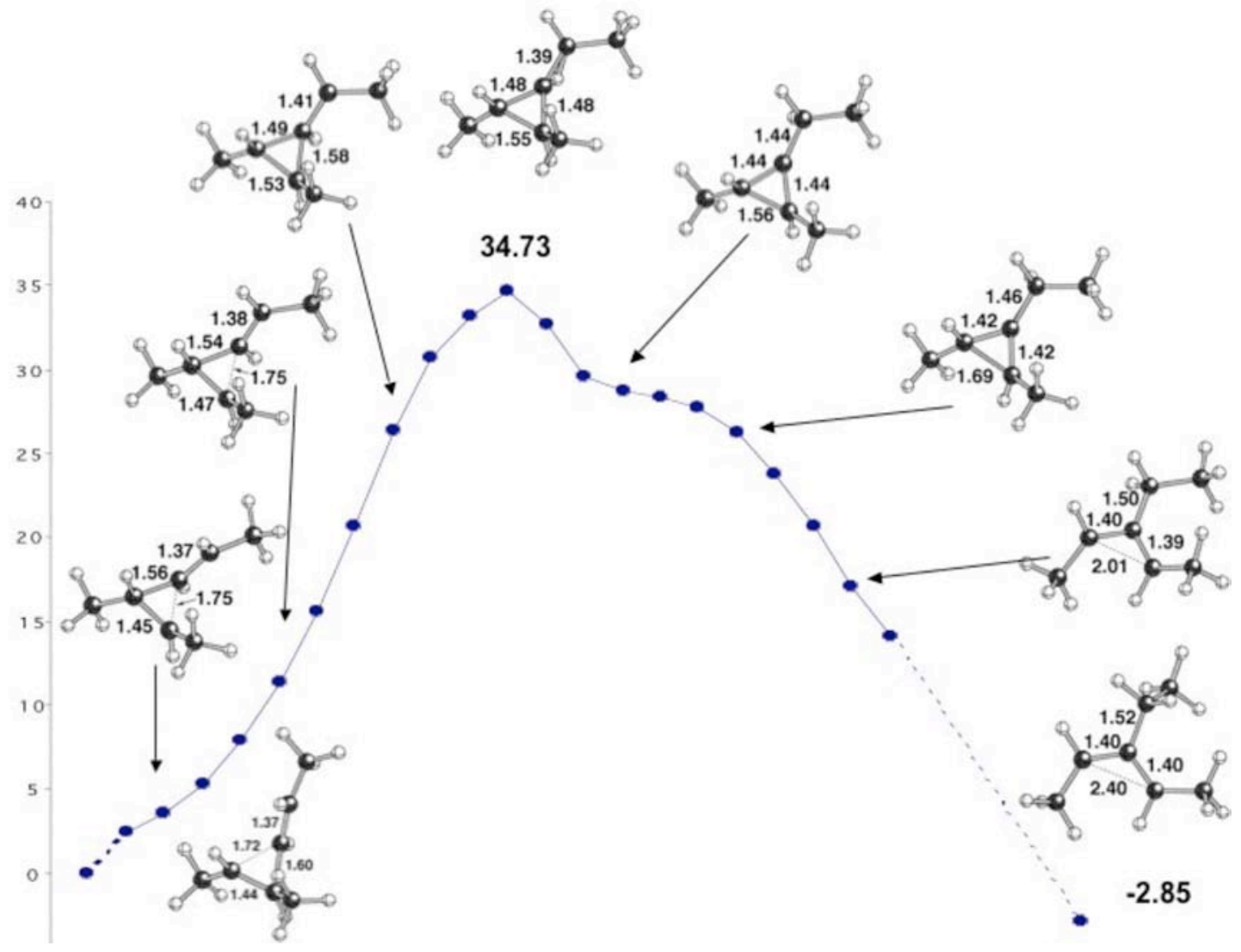


<smiles>CC1CCCC1C</smiles>

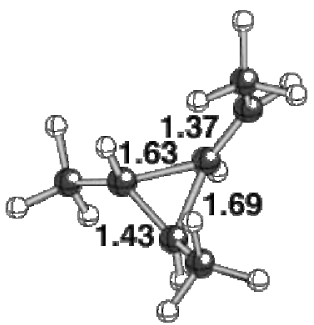

b3lyp/6-31G(d)

\section{Coordinates:}

Standard orientation:

\begin{tabular}{|c|c|c|c|c|c|}
\hline \multirow{2}{*}{$\begin{array}{l}\text { Center } \\
\text { Number }\end{array}$} & \multirow{2}{*}{$\begin{array}{l}\text { Atomic } \\
\text { Number }\end{array}$} & \multirow{2}{*}{\multicolumn{2}{|c|}{$\begin{array}{r}\text { Atomic } \\
\text { Type }\end{array}$}} & \multicolumn{2}{|c|}{ Coordinates (Angstrom } \\
\hline & & & & $X \quad Y$ & $Z$ \\
\hline 1 & 6 & 0 & -0.992180 & -0.184447 & -0.443900 \\
\hline 2 & 6 & 0 & 0.034878 & -0.563851 & 0.767116 \\
\hline 3 & 6 & 0 & -0.622142 & 0.942616 & 0.358786 \\
\hline 4 & 1 & 0 & -1.275084 & 1.159964 & 1.204323 \\
\hline 5 & 6 & 0 & 1.340016 & -0.918607 & 0.538257 \\
\hline 6 & 6 & 0 & 0.254770 & 2.086701 & -0.065883 \\
\hline 7 & 1 & 0 & -0.415798 & 2.907425 & -0.353290 \\
\hline 8 & 1 & 0 & 0.883151 & 1.860015 & -0.928571 \\
\hline 9 & 1 & 0 & 0.876751 & 2.449347 & 0.757132 \\
\hline 10 & 6 & 0 & -2.324242 & -0.889828 & -0.334936 \\
\hline 11 & 1 & 0 & -2.760634 & -0.794380 & 0.663958 \\
\hline 12 & 1 & 0 & -3.015688 & -0.428465 & -1.050498 \\
\hline 13 & 1 & 0 & -2.233609 & -1.949630 & -0.587025 \\
\hline 14 & 1 & 0 & -0.494038 & -0.271378 & -1.408143 \\
\hline 15 & 6 & 0 & 2.235662 & -0.604259 & -0.594310 \\
\hline 16 & 1 & 0 & 1.770423 & -0.074369 & -1.424507 \\
\hline 17 & 1 & 0 & 3.087985 & -0.015760 & -0.219480 \\
\hline 18 & 1 & 0 & 2.679070 & -1.540141 & -0.962951 \\
\hline 19 & 1 & 0 & 1.788444 & -1.550519 & 1.307540 \\
\hline 20 & 1 & 0 & -0.451537 & -0.962056 & 1.650733 \\
\hline
\end{tabular}

Energy: $\mathrm{HF}=-274.2758081$

Zero-Point Energy Correction: 0.182266

Imaginary Frequency: Minimum (No imaginary frequencies) 

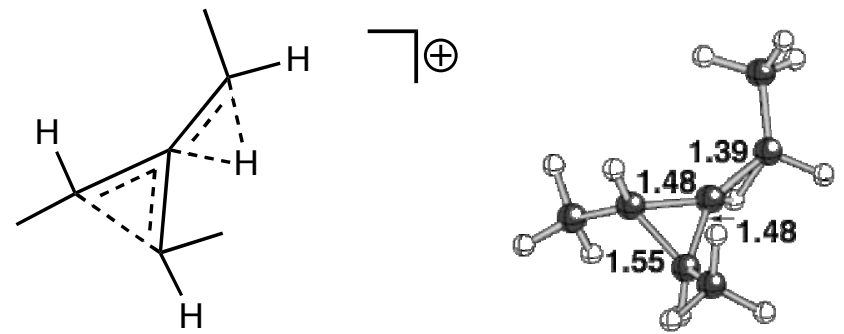

b3lyp/6-31G(d)

\section{Coordinates:}

Standard orientation:

\begin{tabular}{|c|c|c|c|c|c|}
\hline Center & Atomic & & Atomic & Coordinate & e \\
\hline Number & Numbe & & Type & $\begin{array}{ll}X & Y\end{array}$ & $\mathrm{Z}$ \\
\hline 1 & 6 & 0 & -0.676182 & 0.755756 & 0.428682 \\
\hline 2 & 6 & 0 & 23 & -0.169779 & -0.262378 \\
\hline 3 & 6 & 0 & -1.202387 & -0.459126 & -0.371218 \\
\hline 4 & 1 & 0 & -1.668322 & -0.168851 & -1.313356 \\
\hline 5 & 6 & 0 & 85 & -0.667931 & -0.341180 \\
\hline 6 & 6 & 0 & -1.780991 & -1.701888 & 0.280539 \\
\hline 7 & 1 & 0 & -2.839493 & -1.534325 & 0.502411 \\
\hline 8 & 1 & 0 & -1.2 & -1.941778 & 1.218572 \\
\hline 9 & 1 & 0 & & 0.168306 & -1.273073 \\
\hline 10 & 1 & 0 & & -2.567264 & -0.385458 \\
\hline 11 & 6 & & -0.945388 & 2.168438 & -0.064882 \\
\hline 12 & 1 & 0 & -0.944086 & 2.221830 & -1.158960 \\
\hline 13 & 1 & 0 & -1.925728 & 2.499314 & 0.289504 \\
\hline 14 & 1 & 0 & & 2.865899 & 0.316399 \\
\hline 15 & 1 & & -0.6 & 0.6456 & 1.514921 \\
\hline 16 & 6 & 0 & 2.735838 & -0.055049 & 0.293428 \\
\hline 17 & 1 & 0 & 2.527017 & 0.916622 & 0.744792 \\
\hline 18 & 1 & 0 & 3.051973 & -0.750541 & 1.086072 \\
\hline 19 & 1 & & 3.572457 & 0.013972 & -0.408912 \\
\hline 20 & 1 & 0 & 1.674358 & -1.591319 & -0.91085 \\
\hline
\end{tabular}

Energy: $\mathrm{HF}=-274.2261097$

Zero-Point Energy Correction: $\mathbf{0 . 1 7 8 4 7 3}$

Imaginary Frequency: One imaginary frequency $-472.8124 \mathrm{~cm}^{-1}$ 

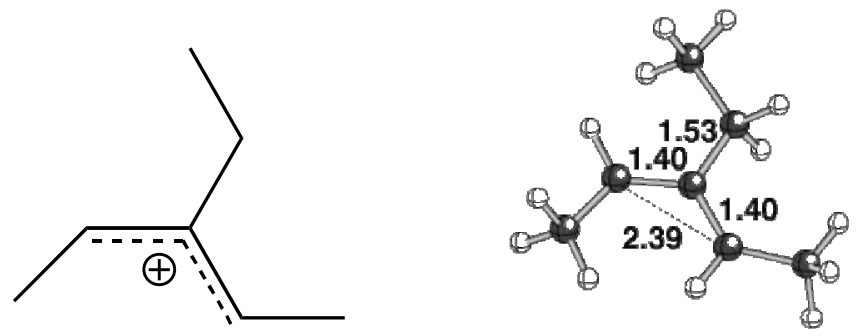

b3lyp/6-31G(d)

\section{Coordinates:}

Standard orientation:

\begin{tabular}{|c|c|c|c|c|c|}
\hline \multirow{2}{*}{$\begin{array}{l}\text { Center } \\
\text { Number }\end{array}$} & \multirow{2}{*}{\multicolumn{2}{|c|}{$\begin{array}{l}\text { Atomic } \\
\text { Number }\end{array}$}} & \multirow{2}{*}{$\begin{array}{r}\text { Atomic } \\
\text { Type }\end{array}$} & \multicolumn{2}{|c|}{ Coordinates (Angstro } \\
\hline & & & & $\begin{array}{ll}\mathrm{X} & \mathrm{Y}\end{array}$ & $\mathrm{Z}$ \\
\hline 1 & 6 & 0 & 0.545265 & 1.258384 & 0.002457 \\
\hline 2 & 6 & 0 & -0.041659 & -0.010436 & $-0.00194 ?$ \\
\hline 3 & 6 & 0 & -1.435534 & -0.080392 & -0.001460 \\
\hline 4 & 1 & 0 & -1.995704 & 0.852162 & 0.000061 \\
\hline 5 & 6 & 0 & 0.802473 & -1.281796 & -0.004292 \\
\hline 6 & 6 & 0 & -2.257523 & -1.297225 & 0.001709 \\
\hline 7 & 1 & 0 & -2.923455 & -1.271006 & 0.880744 \\
\hline 8 & 1 & 0 & -1.697829 & -2.231256 & -0.009927 \\
\hline 9 & 1 & 0 & 0.519258 & -1.873081 & -0.884461 \\
\hline 10 & 1 & 0 & -2.950250 & -1.262428 & $-0.85534 ?$ \\
\hline 11 & 6 & 0 & -0.060267 & 2.603096 & -0.00042 \\
\hline 12 & 1 & & -1.148817 & 2.650602 & 0.00169 \\
\hline 13 & 1 & 0 & 0.329227 & 3.162070 & 0.86472 \\
\hline 14 & 1 & & 0.323993 & 3.152190 & -0.87482 \\
\hline 15 & 1 & 0 & 1.634479 & 1.274738 & 0.00739 \\
\hline 16 & 6 & & 2.321530 & -1.089962 & 0.00240 \\
\hline 17 & 1 & 0 & 2.675300 & -0.551165 & -0.88407 \\
\hline 18 & 1 & 0 & 2.668563 & -0.560531 & 0.89718 \\
\hline 19 & 1 & 0 & 2.807106 & -2.069260 & -0.0005 \\
\hline 20 & 1 & 0 & 0.512413 & -1.883049 & 0.86671 \\
\hline
\end{tabular}

Energy: $H F=-274.2854349$

Zero-Point Energy Correction: $\mathbf{0 . 1 8 2 0 8 0}$

Imaginary Frequency: Minimum (No imaginary frequencies) 


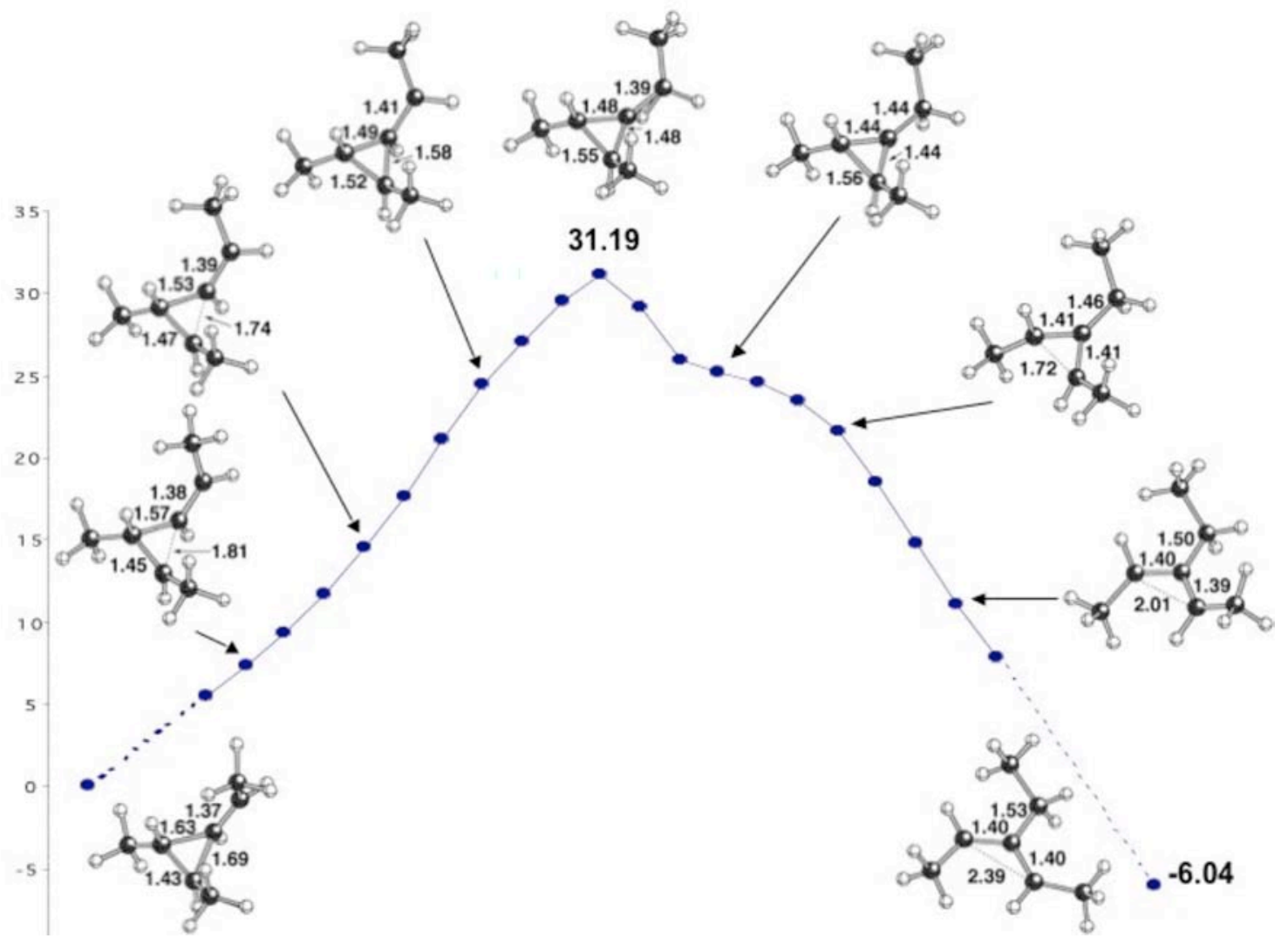


<smiles>CC1CCCC1C</smiles>

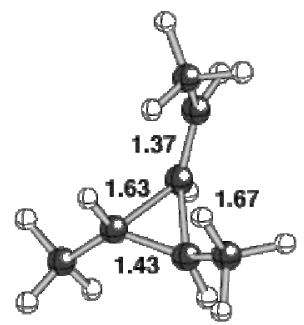

MP2/6-31G(d)

Coordinates:

Standard orientation:

\begin{tabular}{|c|c|c|c|c|c|}
\hline \multirow{2}{*}{$\begin{array}{l}\text { Center } \\
\text { Number }\end{array}$} & \multirow{2}{*}{\multicolumn{2}{|c|}{$\begin{array}{l}\text { Atomic } \\
\text { Number }\end{array}$}} & \multirow{2}{*}{$\begin{array}{l}\text { Atomic } \\
\text { Type }\end{array}$} & \multicolumn{2}{|c|}{ Coordinates (Angstroms) } \\
\hline & & & & $\mathrm{X} \quad \mathrm{Y}$ & $\mathrm{Z}$ \\
\hline 1 & 6 & 0 & -0.995536 & -0.151254 & -0.452586 \\
\hline 2 & 6 & 0 & 0.002275 & -0.563567 & 0.769528 \\
\hline 3 & 6 & 0 & -0.580314 & 0.946166 & 0.358011 \\
\hline 4 & 1 & 0 & -1.235744 & 1.193064 & 1.194465 \\
\hline 5 & 6 & 0 & 1.288245 & -0.962541 & 0.531357 \\
\hline 6 & 6 & 0 & 0.357144 & 2.042939 & -0.054936 \\
\hline 7 & 1 & 0 & -0.266317 & 2.910463 & -0.297919 \\
\hline 8 & 1 & 0 & 0.942622 & 1.797696 & -0.939714 \\
\hline 9 & 1 & 0 & -0.509291 & -0.957023 & 1.641415 \\
\hline 10 & 1 & 0 & 1.022476 & 2.332440 & 0.761249 \\
\hline 11 & 6 & 0 & -2.344686 & -0.809092 & -0.336959 \\
\hline 12 & 1 & 0 & -2.770239 & -0.681990 & 0.661305 \\
\hline 13 & 1 & 0 & -3.017333 & -0.333453 & -1.058052 \\
\hline 14 & 1 & 0 & -2.285460 & -1.873437 & -0.572922 \\
\hline 15 & 1 & 0 & -0.487686 & -0.264994 & -1.409004 \\
\hline 16 & 6 & 0 & 2.190627 & -0.637196 & -0.593817 \\
\hline 17 & 1 & 0 & 1.702935 & -0.180259 & -1.452272 \\
\hline 18 & 1 & 0 & 2.974560 & 0.040639 & -0.225263 \\
\hline 19 & 1 & 0 & 2.708046 & -1.548323 & -0.912020 \\
\hline 20 & 1 & 0 & 1.714895 & -1.627551 & 1.285147 \\
\hline
\end{tabular}

Energy: MP2 $=-273.2595455$

Zero-Point Energy Correction: $\mathbf{0 . 1 8 6 3 6 3}$

Imaginary Frequency: Minimum (No imaginary frequencies) 

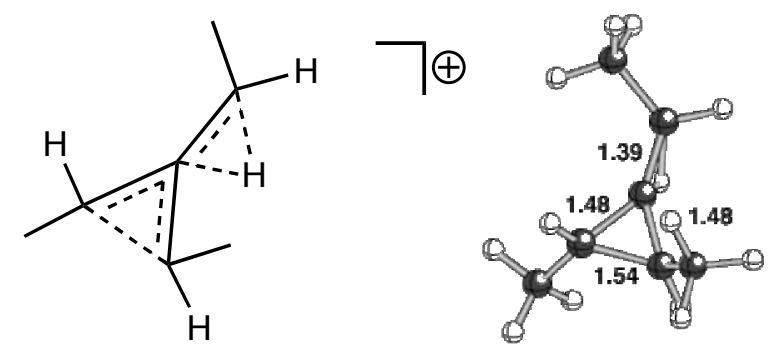

MP2/6-31G(d)

\section{Coordinates:}

Standard orientation:

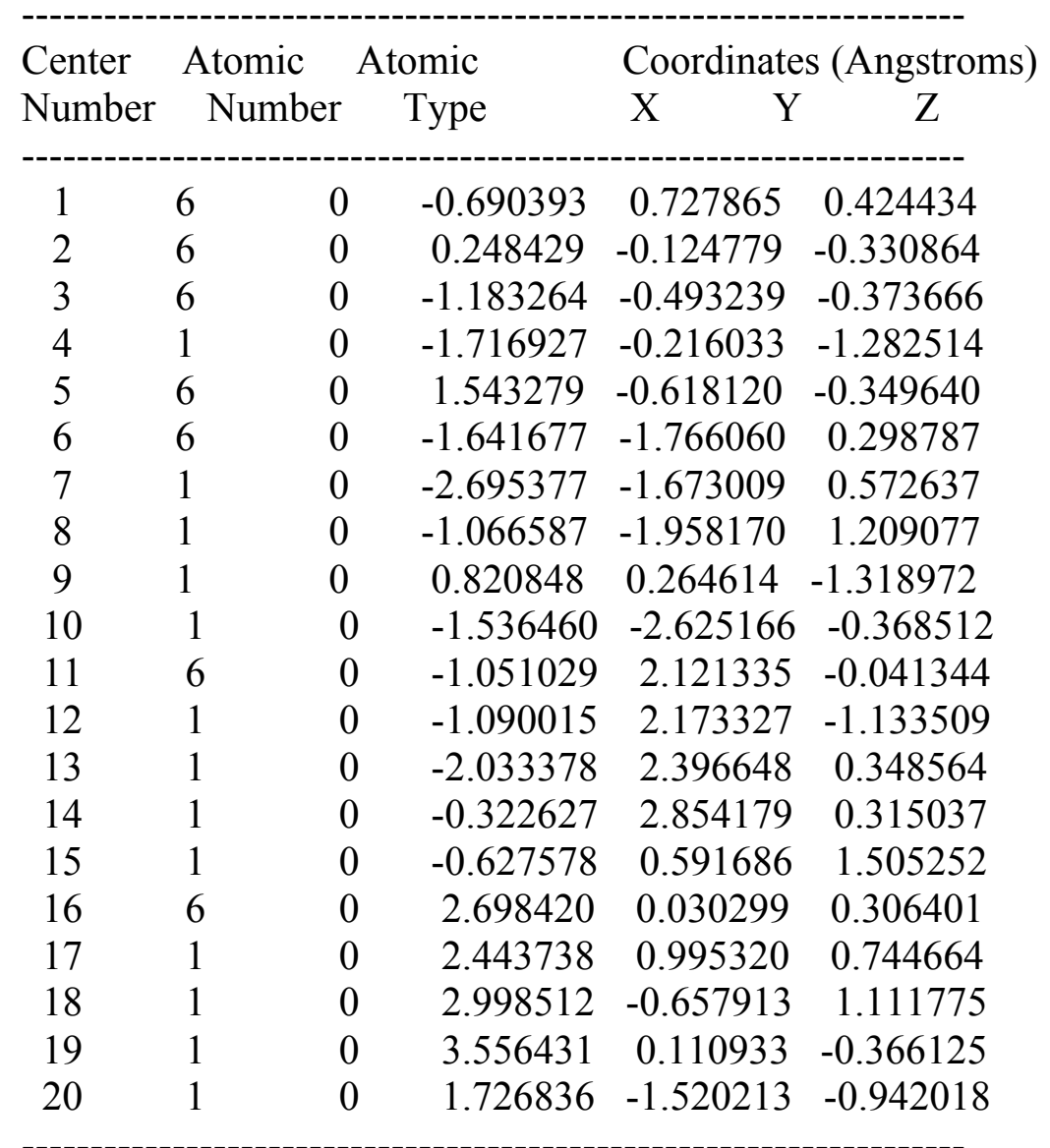

Energy: MP2 $=-273.2074216$

Zero-Point Energy Correction: $\mathbf{0 . 1 8 2 3 1 4}$

Imaginary Frequency: One imaginary frequency $-403.2620 \mathrm{~cm}^{-1}$ 

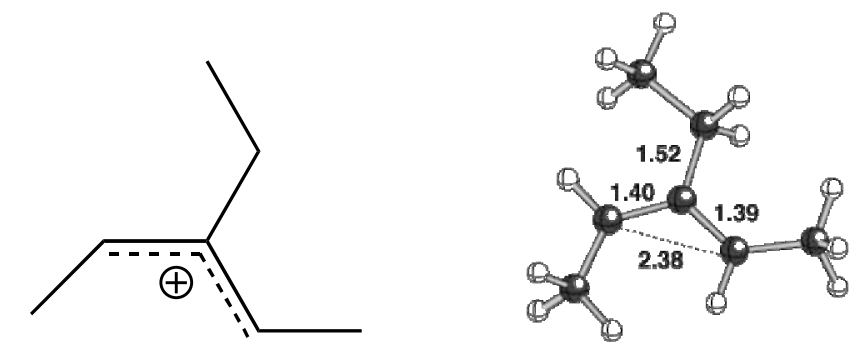

MP2/6-31G(d)

\section{Coordinates:}

Standard orientation:

\begin{tabular}{|c|c|c|c|c|c|}
\hline \multirow{2}{*}{$\begin{array}{l}\text { Center } \\
\text { Number }\end{array}$} & \multirow{2}{*}{\multicolumn{2}{|c|}{$\begin{array}{l}\text { Atomic } \\
\text { Number }\end{array}$}} & \multirow{2}{*}{$\begin{array}{l}\text { Atomic } \\
\text { Type }\end{array}$} & \multicolumn{2}{|c|}{ Coordinates (Angstron } \\
\hline & & & & X $\quad Y$ & $\mathrm{Z}$ \\
\hline 1 & 6 & 0 & 0.519667 & 1.268172 & -0.000037 \\
\hline 2 & 6 & 0 & -0.035781 & -0.013315 & 0.000013 \\
\hline 3 & 6 & 0 & -1.426827 & -0.108248 & -0.000040 \\
\hline 4 & 1 & 0 & -2.009057 & 0.811149 & -0.000087 \\
\hline 5 & 6 & 0 & 0.825718 & -1.263799 & -0.000006 \\
\hline 6 & 6 & 0 & -2.215026 & -1.347208 & 0.000030 \\
\hline 7 & 1 & 0 & -2.886407 & -1.335904 & 0.871139 \\
\hline 8 & 1 & 0 & -1.622667 & -2.259264 & 0.000176 \\
\hline 9 & 1 & 0 & 0.551800 & -1.861588 & -0.876722 \\
\hline 10 & 1 & 0 & -2.886214 & -1.336139 & -0.871239 \\
\hline 11 & 6 & 0 & -0.126146 & 2.595188 & 0.000000 \\
\hline 12 & 1 & 0 & -1.213924 & 2.605386 & -0.000594 \\
\hline 13 & 1 & 0 & 0.241136 & 3.152067 & 0.872755 \\
\hline 14 & 1 & 0 & 0.242121 & 3.152665 & -0.871934 \\
\hline 15 & 1 & 0 & 1.608372 & 1.317025 & 0.000052 \\
\hline 16 & 6 & 0 & 2.333472 & -1.031657 & 0.000003 \\
\hline 17 & 1 & 0 & 2.665202 & -0.490472 & -0.890442 \\
\hline 18 & 1 & 0 & 2.665182 & -0.490394 & 0.890409 \\
\hline 19 & 1 & 0 & 2.842198 & -1.997666 & 0.000056 \\
\hline 20 & 1 & 0 & 0.551803 & -1.861668 & 0.876655 \\
\hline
\end{tabular}

Energy: MP2 $=-273.2591991$

Zero-Point Energy Correction: $\mathbf{0 . 1 8 5 7 3 3}$

Imaginary Frequency: Minimum (No imaginary frequencies) 


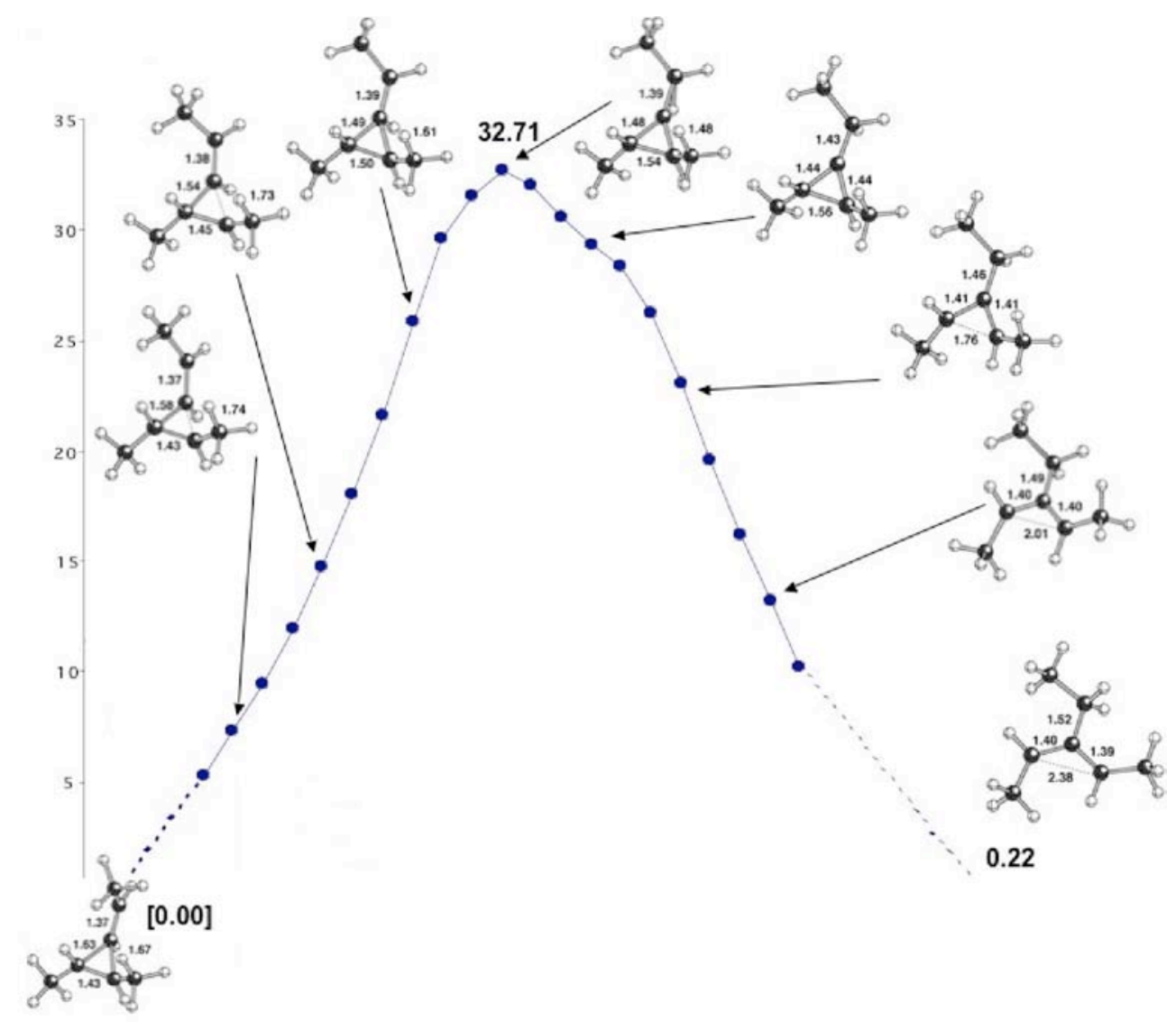



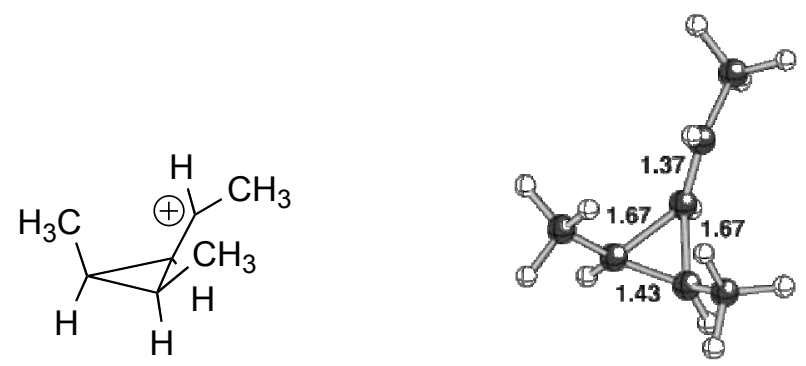

b3lyp/6-31G(d)

\section{Coordinates:}

Standard orientation:

\begin{tabular}{|c|c|c|c|c|c|}
\hline \multirow{2}{*}{$\begin{array}{l}\text { Center } \\
\text { Number }\end{array}$} & \multirow{2}{*}{\multicolumn{2}{|c|}{$\begin{array}{l}\text { Atomic } \\
\text { Number }\end{array}$}} & \multirow{2}{*}{$\begin{array}{l}\text { Atomic } \\
\text { Type }\end{array}$} & \multicolumn{2}{|c|}{ Coordinates (Angstroms } \\
\hline & & & & X $\quad Y$ & Z \\
\hline 1 & 6 & 0 & -1.016177 & -0.717253 & 0.609128 \\
\hline 2 & 6 & 0 & 0.481523 & 0.000099 & 0.752813 \\
\hline 3 & 6 & 0 & -1.015744 & 0.717235 & 0.609171 \\
\hline 4 & 1 & 0 & -1.162235 & 1.193305 & 1.578227 \\
\hline 5 & 6 & 0 & 1.351534 & 0.000094 & -0.301521 \\
\hline 6 & 6 & 0 & -1.344275 & 1.598843 & -0.568590 \\
\hline 7 & 1 & 0 & -2.414634 & 1.832875 & -0.511663 \\
\hline 8 & 1 & 0 & -1.165133 & 1.129891 & -1.537956 \\
\hline 9 & 1 & 0 & 0.871042 & -0.000364 & 1.765288 \\
\hline 10 & 1 & 0 & -0.797362 & 2.544154 & -0.519125 \\
\hline 11 & 6 & 0 & -1.344617 & -1.598720 & -0.568672 \\
\hline 12 & 1 & 0 & -0.798502 & -2.544465 & -0.518710 \\
\hline 13 & 1 & 0 & -2.415211 & -1.831893 & -0.512359 \\
\hline 14 & 1 & 0 & -1.164473 & -1.130084 & -1.537994 \\
\hline 15 & 1 & 0 & -1.162224 & -1.193350 & 1.578221 \\
\hline 16 & 1 & 0 & 0.953542 & 0.000490 & -1.316017 \\
\hline 17 & 6 & 0 & 2.823761 & -0.000276 & -0.181436 \\
\hline 18 & 1 & 0 & 3.232090 & -0.872713 & -0.713701 \\
\hline 19 & 1 & 0 & 3.232100 & 0.873489 & -0.711557 \\
\hline 20 & 1 & 0 & 3.174978 & -0.001465 & 0.851989 \\
\hline
\end{tabular}

Energy: $\mathrm{HF}=-274.276833$

Zero-Point Energy Correction: $\mathbf{0 . 1 8 2 3 0 7}$

Imaginary Frequency: Minimum (No imaginary frequencies) 

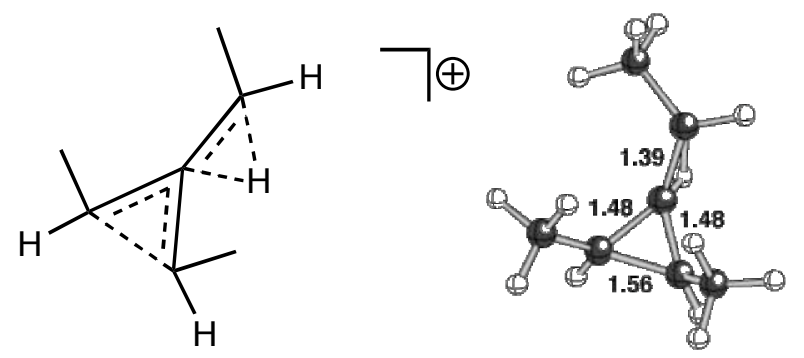

b31yp/6-31G(d)

\section{Coordinates:}

Standard orientation:

\begin{tabular}{|c|c|c|c|c|}
\hline \multirow{2}{*}{$\begin{array}{l}\text { Center } \\
\text { Number }\end{array}$} & \multirow{2}{*}{$\begin{array}{l}\text { Atomic } \\
\text { Number }\end{array}$} & \multirow{2}{*}{$\begin{array}{r}\text { Atomic } \\
\text { Type }\end{array}$} & \multicolumn{2}{|c|}{ Coordinates (Angstroms) } \\
\hline & & & X $\quad Y$ & $\mathrm{Z}$ \\
\hline 1 & 6 & 0.596751 & 0.833520 & 0.673031 \\
\hline 2 & 6 & -0.249677 & -0.357593 & 0.473165 \\
\hline 3 & 6 & 1.204790 & -0.598232 & 0.548008 \\
\hline 4 & 1 & 1.573603 & -0.961914 & 1.506206 \\
\hline 5 & 6 & -1.453398 & -0.826183 & -0.036301 \\
\hline 6 & 6 & 1.964440 & -1.112745 & -0.660433 \\
\hline 7 & 1 & 3.008059 & -0.788863 & -0.599029 \\
\hline 8 & 1 & 1.547817 & -0.744712 & -1.602471 \\
\hline 9 & 1 & -1.057150 & -0.811807 & 1.316754 \\
\hline 10 & 1 & 1.953919 & -2.206987 & -0.685241 \\
\hline 11 & 6 & 0.695370 & 1.903136 & -0.399339 \\
\hline 12 & 1 & -0.083148 & 2.661008 & -0.266122 \\
\hline 13 & 1 & 1.665358 & 2.404306 & -0.323911 \\
\hline 14 & 1 & 0.604027 & 1.490289 & -1.408064 \\
\hline 15 & 1 & 0.655191 & 1.205396 & 1.695314 \\
\hline 16 & 1 & -1.511506 & -1.895688 & -0.258449 \\
\hline 17 & 6 & -2.663875 & 0.002056 & -0.293367 \\
\hline 18 & 1 & -2.797567 & 0.021447 & -1.386202 \\
\hline 19 & 1 & -3.565008 & -0.464019 & 0.117938 \\
\hline 20 & 1 & -2.559999 & 1.027790 & 0.064693 \\
\hline
\end{tabular}

Energy: $\mathrm{HF}=-274.2253055$

Zero-Point Energy Correction: $\mathbf{0 . 1 7 8 5 2 0}$

Imaginary Frequency: One imaginary frequency $-508.8895 \mathrm{~cm}^{-1}$ 
b3lyp/6-31G(d)
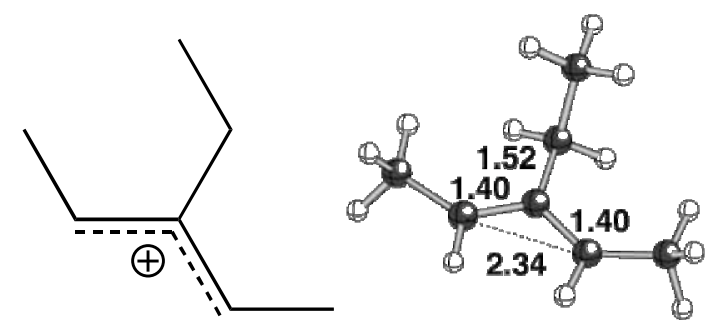

\section{Coordinates:}

Standard orientation:

\begin{tabular}{|c|c|c|c|c|c|}
\hline \multirow{2}{*}{$\begin{array}{l}\text { Center } \\
\text { Number }\end{array}$} & \multirow{2}{*}{\multicolumn{2}{|c|}{$\begin{array}{l}\text { Atomic } \\
\text { Number }\end{array}$}} & \multirow{2}{*}{$\begin{array}{l}\text { Atomic } \\
\text { Type }\end{array}$} & \multicolumn{2}{|c|}{ Coordinates (Angstrom } \\
\hline & & & & X $\quad Y$ & Z \\
\hline 1 & 6 & 0 & -1.169025 & -1.051213 & -0.131917 \\
\hline 2 & 6 & 0 & 0.000436 & -0.338718 & 0.145533 \\
\hline 3 & 6 & 0 & 1.170598 & -1.049962 & -0.132207 \\
\hline 4 & 1 & 0 & 1.055596 & -2.071689 & -0.496525 \\
\hline 5 & 6 & 0 & -0.000277 & 1.087828 & 0.657688 \\
\hline 6 & 6 & 0 & 2.558563 & -0.590006 & 0.008400 \\
\hline 7 & 1 & 0 & 3.084297 & -1.262564 & 0.706996 \\
\hline 8 & 1 & 0 & 2.669717 & 0.441946 & 0.339306 \\
\hline 9 & 1 & 0 & 0.871761 & 1.239819 & 1.300994 \\
\hline 10 & 1 & 0 & 3.083085 & -0.735080 & -0.949482 \\
\hline 11 & 6 & 0 & -2.557427 & -0.592609 & 0.008820 \\
\hline 12 & 1 & 0 & -3.080238 & -0.734173 & -0.950595 \\
\hline 13 & 1 & 0 & -3.084076 & -1.267924 & 0.703928 \\
\hline 14 & 1 & 0 & -2.669498 & 0.438204 & 0.343001 \\
\hline 15 & 1 & 0 & -1.052985 & -2.072791 & -0.496321 \\
\hline 16 & 1 & 0 & -0.871164 & 1.238225 & 1.302933 \\
\hline 17 & 6 & 0 & -0.002446 & 2.131195 & -0.476580 \\
\hline 18 & 1 & 0 & -0.887584 & 2.032677 & -1.113977 \\
\hline 19 & 1 & 0 & -0.003362 & 3.139421 & -0.053319 \\
\hline 20 & 1 & 0 & 0.881926 & 2.034834 & -1.115364 \\
\hline
\end{tabular}

Energy: $\mathrm{HF}=-274.2904158$

Zero-Point Energy Correction: 0.182043

Imaginary Frequency: Minimum (No imaginary frequencies) 


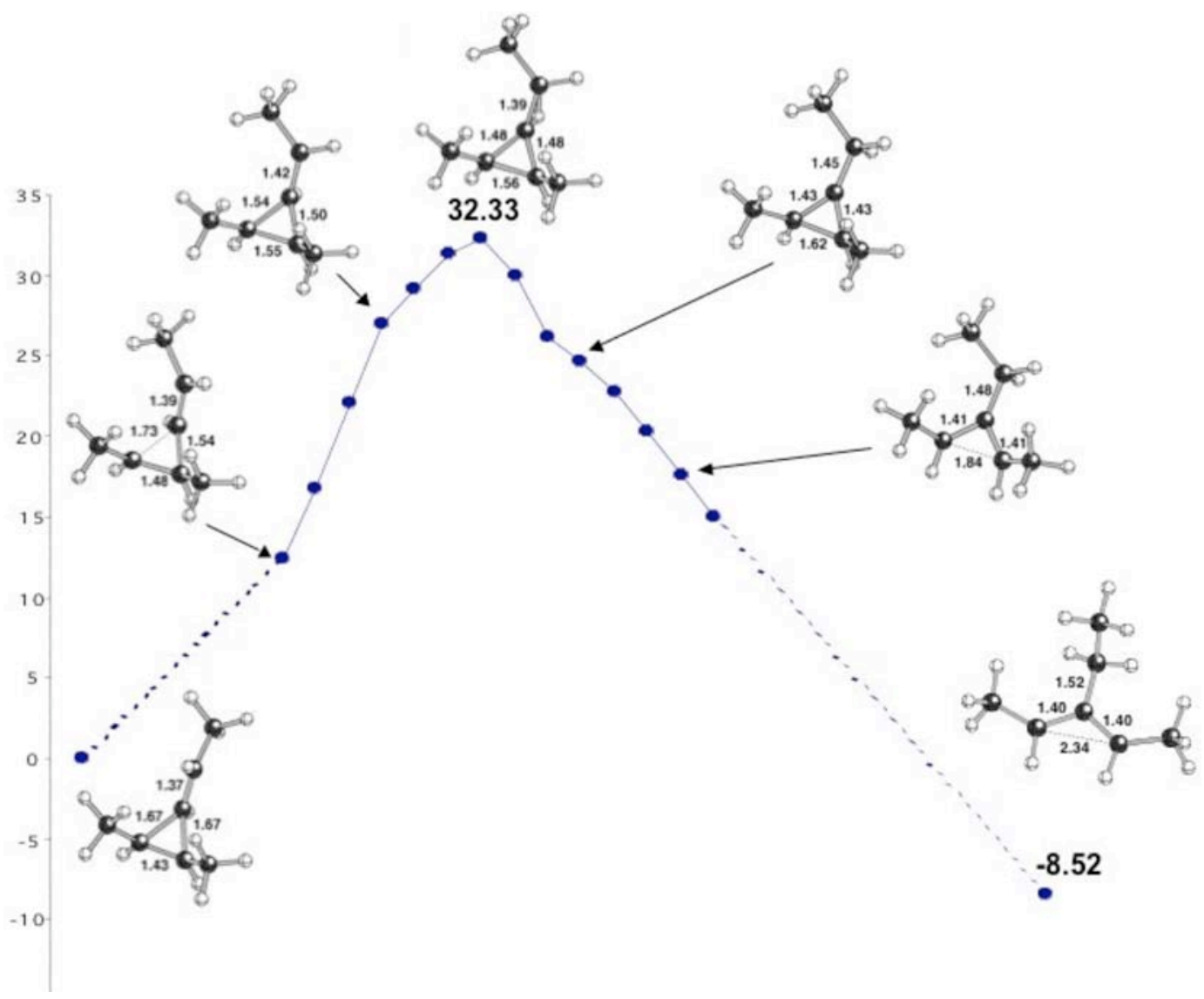




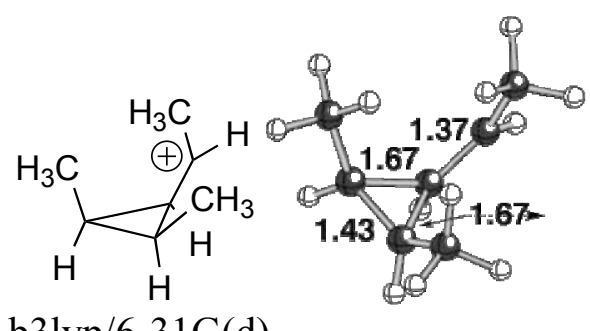

b3lyp/6-31G(d)

\section{Coordinates:}

Standard orientation:

\begin{tabular}{|c|c|c|c|c|c|}
\hline \multirow{2}{*}{$\begin{array}{l}\text { Center } \\
\text { Number }\end{array}$} & \multirow{2}{*}{\multicolumn{2}{|c|}{$\begin{array}{l}\text { Atomic } \\
\text { Number }\end{array}$}} & \multirow{2}{*}{$\begin{array}{r}\text { Atomic } \\
\text { Type }\end{array}$} & \multicolumn{2}{|c|}{ Coordinates (Angstrom } \\
\hline & & & & $\begin{array}{ll}\mathrm{X} & \mathrm{Y}\end{array}$ & $\mathrm{Z}$ \\
\hline 1 & 6 & 0 & -1.049119 & -0.717303 & 0.470408 \\
\hline 2 & 6 & 0 & 0.287922 & -0.000627 & 1.169967 \\
\hline 3 & 6 & 0 & -1.049696 & 0.717085 & 0.470408 \\
\hline 4 & 1 & 0 & -1.536671 & 1.188641 & 1.323502 \\
\hline 5 & 6 & 0 & 1.553462 & 0.000213 & 0.641149 \\
\hline 6 & 6 & 0 & -0.941912 & 1.614528 & -0.733512 \\
\hline 7 & 1 & 0 & -1.963280 & 1.921568 & -0.992355 \\
\hline 8 & 6 & 0 & 2.055908 & 0.000238 & -0.747981 \\
\hline 9 & 1 & 0 & 2.715085 & 0.871950 & -0.878667 \\
\hline 10 & 1 & 0 & 2.347040 & 0.000940 & 1.391622 \\
\hline 11 & 1 & 0 & -0.513696 & 1.135874 & -1.614434 \\
\hline 12 & 1 & 0 & 2.716202 & -0.870724 & -0.878180 \\
\hline 13 & 1 & 0 & -0.378298 & 2.523014 & -0.504404 \\
\hline 14 & 1 & 0 & 1.300008 & -0.000547 & -1.528030 \\
\hline 15 & 6 & 0 & -0.941565 & -1.614395 & -0.733924 \\
\hline 16 & 1 & 0 & -0.377162 & -2.522547 & -0.505464 \\
\hline 17 & 1 & 0 & -1.962858 & -1.921980 & -0.992326 \\
\hline 18 & 1 & 0 & -0.514138 & -1.135176 & -1.614935 \\
\hline 19 & 1 & 0 & -1.536614 & -1.189025 & 1.323124 \\
\hline 20 & 1 & 0 & 0.214385 & -0.000423 & 2.251455 \\
\hline
\end{tabular}

Energy: $\mathrm{HF}=-274.2696919$

Zero-Point Energy Correction: 0.182513

Imaginary Frequency: Minimum (No imaginary frequencies) 
b3lyp/6-31G(d)
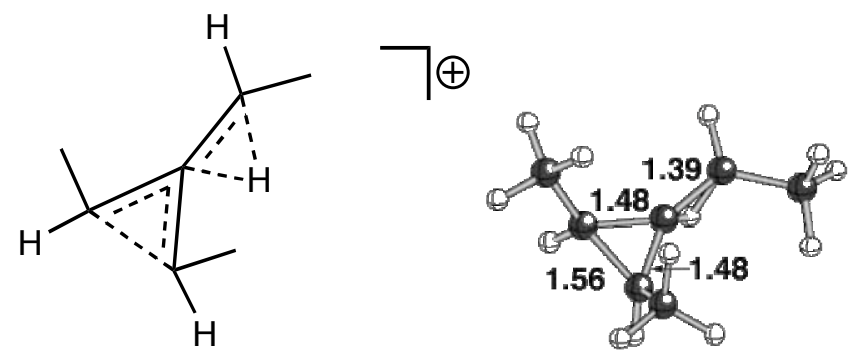

Coordinates:

Standard orientation:

\begin{tabular}{|c|c|c|c|c|c|}
\hline \multirow{2}{*}{$\begin{array}{l}\text { Center } \\
\text { Number }\end{array}$} & \multirow{2}{*}{$\begin{array}{l}\text { Atomic } \\
\text { Number }\end{array}$} & \multirow{2}{*}{\multicolumn{2}{|c|}{$\begin{array}{l}\text { Atomic } \\
\text { Type }\end{array}$}} & \multicolumn{2}{|c|}{ Coordinates (Angstroms) } \\
\hline & & & & X $\quad Y$ & $\mathrm{Z}$ \\
\hline 1 & 6 & 0 & -1.204940 & -0.597975 & 0.547994 \\
\hline 2 & 6 & 0 & 0.249551 & -0.357537 & 0.473262 \\
\hline 3 & 6 & 0 & -0.596653 & 0.833736 & 0.672940 \\
\hline 4 & 1 & 0 & -0.655266 & 1.205658 & 1.695194 \\
\hline 5 & 6 & 0 & 1.453092 & -0.826406 & -0.036325 \\
\hline 6 & 6 & 0 & -0.694840 & 1.903341 & -0.399474 \\
\hline 7 & 1 & 0 & -1.664806 & 2.404599 & -0.324345 \\
\hline 8 & 6 & 0 & 2.663742 & 0.001712 & -0.293313 \\
\hline 9 & 1 & 0 & 3.564738 & -0.464754 & 0.117863 \\
\hline 10 & 1 & 0 & 1.510947 & -1.895917 & -0.258527 \\
\hline 11 & 1 & 0 & -0.603216 & 1.490494 & -1.408169 \\
\hline 12 & 1 & 0 & 1.057240 & -0.811984 & 1.316678 \\
\hline 13 & 1 & 0 & 2.797435 & 0.021615 & -1.386070 \\
\hline 14 & 1 & 0 & 0.083708 & 2.661140 & -0.266007 \\
\hline 15 & 1 & 0 & 2.560080 & 1.027286 & 0.065303 \\
\hline 16 & 6 & 0 & -1.964484 & -1.112825 & -0.660369 \\
\hline 17 & 1 & 0 & -1.953115 & -2.207054 & -0.685401 \\
\hline 18 & 1 & 0 & -3.008334 & -0.789774 & -0.598667 \\
\hline 19 & 1 & 0 & -1.548341 & -0.744279 & -1.602415 \\
\hline 20 & 1 & 0 & -1.573888 & -0.961306 & 1.506272 \\
\hline
\end{tabular}

Energy: -274.2253055

Zero-Point Energy Correction: 0.178519

Imaginary Frequency: One imaginary frequency $-509.0484 \mathrm{~cm}^{-1}$ 

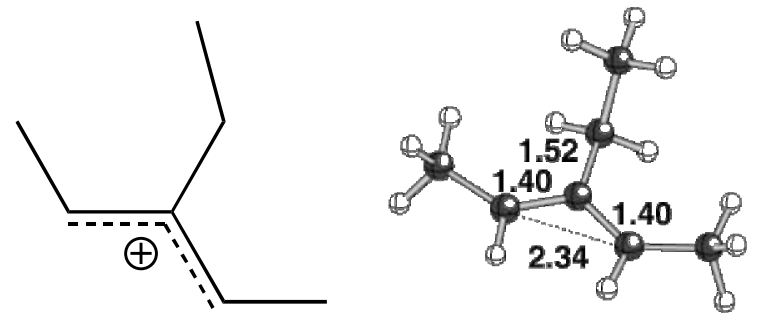

b3lyp/6-31G(d)

Coordinates:

Standard orientation:

\begin{tabular}{|c|c|c|c|c|}
\hline \multirow{2}{*}{$\begin{array}{l}\text { Center } \\
\text { Number }\end{array}$} & \multirow{2}{*}{$\begin{array}{l}\text { Atomic } \\
\text { Number }\end{array}$} & \multirow{2}{*}{$\begin{array}{r}\text { Atomic } \\
\text { Type }\end{array}$} & \multicolumn{2}{|c|}{ Coordinates (Angstroms } \\
\hline & & & X $\quad Y$ & Z \\
\hline 1 & 6 & -1.170341 & -1.050160 & -0.131953 \\
\hline 2 & 6 & -0.000266 & -0.338654 & 0.145579 \\
\hline 3 & 6 & 1.169298 & -1.050960 & -0.131985 \\
\hline 4 & 1 & 1.053408 & -2.072579 & -0.496326 \\
\hline 5 & 6 & 0.000268 & 1.087898 & 0.657737 \\
\hline 6 & 6 & 2.557661 & -0.592194 & 0.008662 \\
\hline 7 & 1 & 3.083921 & -1.266748 & 0.704831 \\
\hline 8 & 6 & 0.001529 & 2.131157 & -0.476597 \\
\hline 9 & 1 & 0.002126 & 3.139430 & -0.053439 \\
\hline 10 & 1 & 0.871470 & 1.238578 & 1.302497 \\
\hline 11 & 1 & 2.669655 & 0.438950 & 0.341816 \\
\hline 12 & 1 & -0.871441 & 1.239726 & 1.301493 \\
\hline 13 & 1 & 0.886450 & 2.033022 & -1.114362 \\
\hline 14 & 1 & 3.080932 & -0.734933 & -0.950307 \\
\hline 15 & 1 & -0.883049 & 2.034352 & -1.115039 \\
\hline 16 & 6 & -2.558432 & -0.590562 & 0.008519 \\
\hline 17 & 1 & -3.081367 & -0.732415 & -0.950790 \\
\hline 18 & 1 & -3.085459 & -1.265173 & 0.704024 \\
\hline 19 & 1 & -2.669899 & 0.440491 & 0.342130 \\
\hline 20 & 1 & -1.055053 & -2.071849 & -0.496303 \\
\hline
\end{tabular}

Energy: $\mathrm{HF}=-274.2904158$

Zero-Point Energy Correction: 0.182043

Imaginary Frequency: Minimum (No imaginary frequencies) 


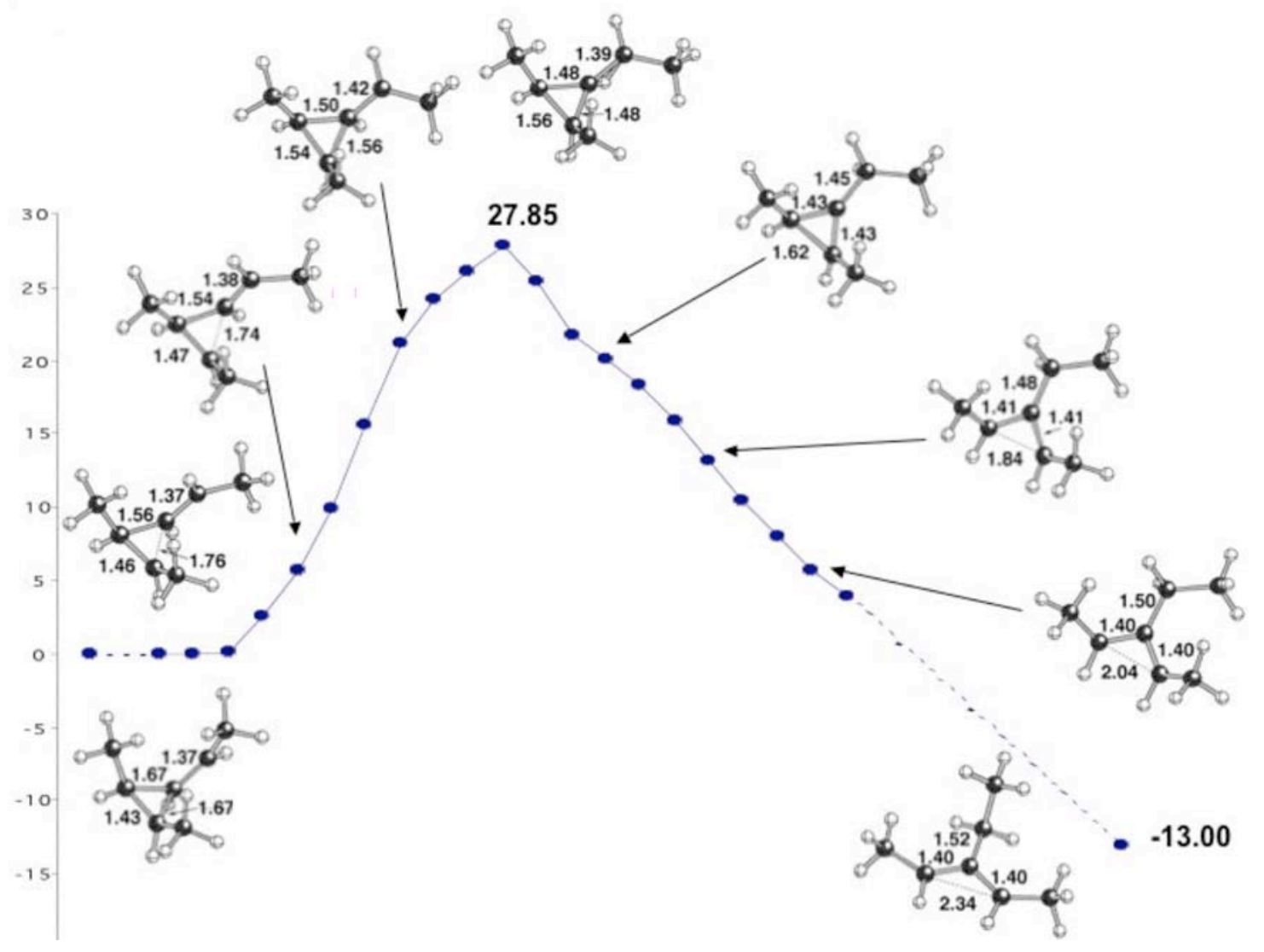


<smiles>CC1=CC(C)C1C</smiles>

b3lyp/6-31G(d)

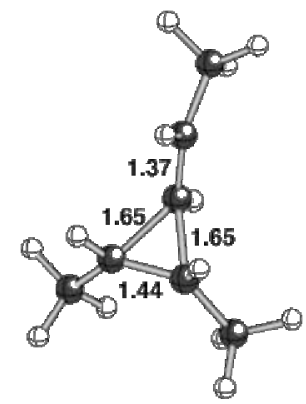

\section{Coordinates:}

Standard orientation:

\begin{tabular}{|c|c|c|c|c|c|}
\hline \multirow{2}{*}{$\begin{array}{l}\text { Center } \\
\text { Number }\end{array}$} & \multirow{2}{*}{\multicolumn{2}{|c|}{$\begin{array}{l}\text { Atomic } \\
\text { Number }\end{array}$}} & \multirow{2}{*}{$\begin{array}{l}\text { Atomic } \\
\text { Type }\end{array}$} & \multicolumn{2}{|c|}{ Coordinates (Angstrom } \\
\hline & & & & X $\quad \mathrm{Y}$ & $\mathrm{Z}$ \\
\hline 1 & 6 & 0 & -0.831868 & 0.718335 & 0.555934 \\
\hline 2 & 6 & 0 & 0.438208 & -0.000185 & -0.223568 \\
\hline 3 & 6 & 0 & -0.832147 & -0.718317 & 0.555830 \\
\hline 4 & 6 & 0 & 1.669211 & -0.000200 & 0.374564 \\
\hline 5 & 1 & 0 & 0.351167 & -0.000085 & -1.305356 \\
\hline 6 & 6 & 0 & -1.673793 & 1.599444 & -0.333376 \\
\hline 7 & 1 & 0 & -2.604335 & 1.824297 & 0.203297 \\
\hline 8 & 1 & 0 & -1.168304 & 2.547495 & -0.535479 \\
\hline 9 & 1 & o & -1.938726 & 1.128651 & -1.282357 \\
\hline 10 & 6 & 0 & -1.674446 & -1.599092 & -0.333426 \\
\hline 11 & 1 & 0 & -1.169910 & -2.547896 & -0.534334 \\
\hline 12 & 1 & 0 & -2.605722 & -1.822342 & 0.202641 \\
\hline 13 & 1 & 0 & -1.938023 & -1.128787 & -1.283033 \\
\hline 14 & 1 & 0 & -0.486013 & 1.194263 & 1.470825 \\
\hline 15 & 1 & 0 & -0.486323 & -1.194375 & 1.470673 \\
\hline 16 & 6 & 0 & 2.964857 & -0.000108 & -0.333282 \\
\hline 17 & 1 & 0 & 2.865548 & -0.000122 & -1.420285 \\
\hline 18 & 1 & 0 & 3.553839 & 0.873048 & -0.014104 \\
\hline 19 & 1 & 0 & 3.553990 & -0.873143 & -0.014070 \\
\hline 20 & 1 & 0 & 1.712676 & -0.000273 & 1.465516 \\
\hline
\end{tabular}

Energy: $\mathrm{HF}=-274.2814985$

Zero-Point Energy Correction: $\mathbf{0 . 1 8 2 2 6 5}$

Imaginary Frequency: Minimum (No imaginary frequencies) 

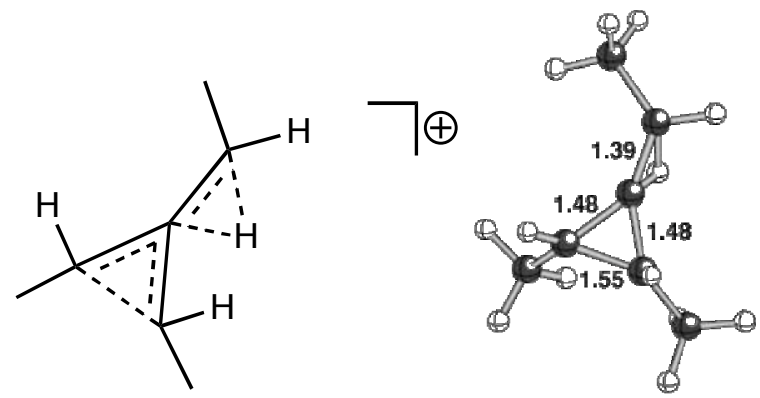

b3lyp/6-31G(d)

\section{Coordinates:}

Standard orientation:

\begin{tabular}{|c|c|c|c|c|c|}
\hline \multirow{2}{*}{$\begin{array}{l}\text { Center } \\
\text { Number }\end{array}$} & \multirow{2}{*}{$\begin{array}{l}\text { Atomic } \\
\text { Number }\end{array}$} & \multirow{2}{*}{\multicolumn{2}{|c|}{$\begin{array}{c}\text { Atomic } \\
\mathrm{r} \text { Type }\end{array}$}} & \multicolumn{2}{|c|}{ Coordinates (Angstror } \\
\hline & & & & X $\quad Y$ & Z \\
\hline 1 & 6 & 0 & -0.546793 & 0.774952 & 0.612755 \\
\hline 2 & 6 & 0 & 0.333919 & -0.326236 & 164970 \\
\hline 3 & 6 & 0 & -1.044944 & -0.689167 & 0.564742 \\
\hline 4 & 6 & 0 & 1.629170 & -0.753535 & -0.085210 \\
\hline 5 & 1 & 0 & 0.663128 & -0.429294 & -1.047785 \\
\hline 6 & 6 & 0 & -1.071206 & 1.852519 & -0.324289 \\
\hline 7 & 1 & 0 & -2.0020 & 2.263500 & 0.076928 \\
\hline 8 & 1 & 0 & -0.349007 & 2.670722 & -0.405472 \\
\hline 9 & 1 & 0 & -1.273618 & 1.474957 & -1.330368 \\
\hline 10 & 6 & 0 & -2.096900 & -1.173428 & -0.420200 \\
\hline 11 & 1 & 0 & -2.011 & -2.254968 & -0.563959 \\
\hline 12 & 1 & 0 & -3.093849 & -0.963839 & -0.022094 \\
\hline 13 & 1 & 0 & -2.013760 & -0.691063 & -1.398179 \\
\hline 14 & 1 & 0 & -0.289017 & 1.142649 & 1.607361 \\
\hline 15 & 1 & 0 & -1.078150 & -1.197092 & 1.530062 \\
\hline 16 & 6 & 0 & 2.830684 & 0.124156 & -0.146870 \\
\hline 17 & 1 & 0 & 2.5846 & 1.185465 & -0.081194 \\
\hline 18 & 1 & 0 & 3.449280 & -0.148655 & 0.722355 \\
\hline 19 & 1 & 0 & 3.437879 & -0.082183 & -1.033928 \\
\hline 20 & 1 & 0 & 1.772447 & -1.825761 & -0.249113 \\
\hline
\end{tabular}

Energy: $\mathrm{HF}=-274.2236127$

Zero-Point Energy Correction: $\mathbf{0 . 1 7 8 7 6 5}$

Imaginary Frequency: One imaginary frequency $-424.9317 \mathrm{~cm}^{-1}$ 

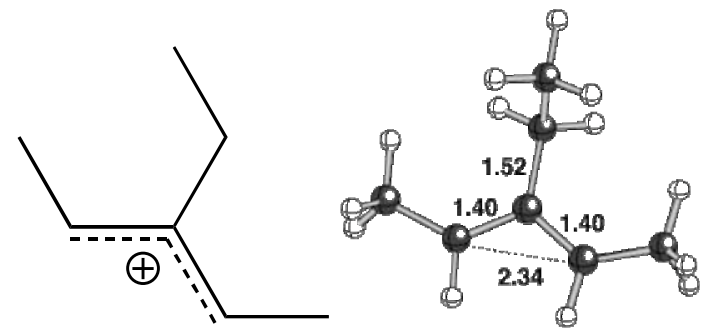

b3lyp/6-31G(d)

\section{Coordinates:}

Standard orientation:

\begin{tabular}{|c|c|c|c|c|c|}
\hline \multirow{2}{*}{$\begin{array}{l}\text { Center } \\
\text { Number }\end{array}$} & \multirow{2}{*}{$\begin{array}{l}\text { Atomic } \\
\text { Number }\end{array}$} & \multirow{2}{*}{\multicolumn{2}{|c|}{$\begin{array}{r}\text { Atomic } \\
\quad \text { Type }\end{array}$}} & \multicolumn{2}{|c|}{ Coordinates (Angstrom } \\
\hline & & & & $\begin{array}{ll}X & Y\end{array}$ & $Y \quad Z$ \\
\hline 1 & 6 & 0 & 1.173406 & -1.047822 & -0.132543 \\
\hline 2 & 6 & 0 & 0.002026 & -0.338664 & 0.145296 \\
\hline 3 & 6 & 0 & -1.166216 & -1.053276 & -0.131882 \\
\hline 4 & 6 & 0 & -0.001592 & 1.087846 & 0.657555 \\
\hline 5 & 1 & 0 & 0.871919 & 1.242724 & 1.298128 \\
\hline 6 & 6 & 0 & 2.560627 & -0.585652 & 0.008176 \\
\hline 7 & 1 & 0 & 3.087461 & -1.257357 & 0.706733 \\
\hline 8 & 1 & 0 & 3.085327 & -0.729746 & -0.949760 \\
\hline 9 & 1 & 0 & 2.670090 & 0.446481 & 0.339102 \\
\hline 10 & 6 & 0 & -2.555373 & -0.597184 & $4 \quad 0.009519$ \\
\hline 11 & 1 & 0 & -3.078206 & -0.739223 & -0.949839 \\
\hline 12 & 1 & 0 & -3.080665 & -1.273784 & 0.704383 \\
\hline 13 & 1 & 0 & -2.669172 & 0.433280 & 0.344169 \\
\hline 14 & 1 & 0 & 1.060082 & -2.069703 & $3-0.496966$ \\
\hline 15 & 1 & 0 & -1.048484 & -2.074558 & $8-0.496579$ \\
\hline 16 & 6 & 0 & -0.010839 & 2.131296 & $6-0.476536$ \\
\hline 17 & 1 & 0 & 0.871423 & 2.037502 & -1.118637 \\
\hline 18 & 1 & 0 & -0.898040 & 2.030422 & $2-1.110686$ \\
\hline 19 & 1 & 0 & -0.013011 & 3.139481 & $1-0.053171$ \\
\hline 20 & 1 & 0 & -0.870953 & 1.235214 & $4 \quad 1.305614$ \\
\hline
\end{tabular}

Energy: $H F=-274.2904159$

Zero-Point Energy Correction: $\mathbf{0 . 1 8 2 0 4 5}$

Imaginary Frequency: Minimum (No imaginary frequencies) 


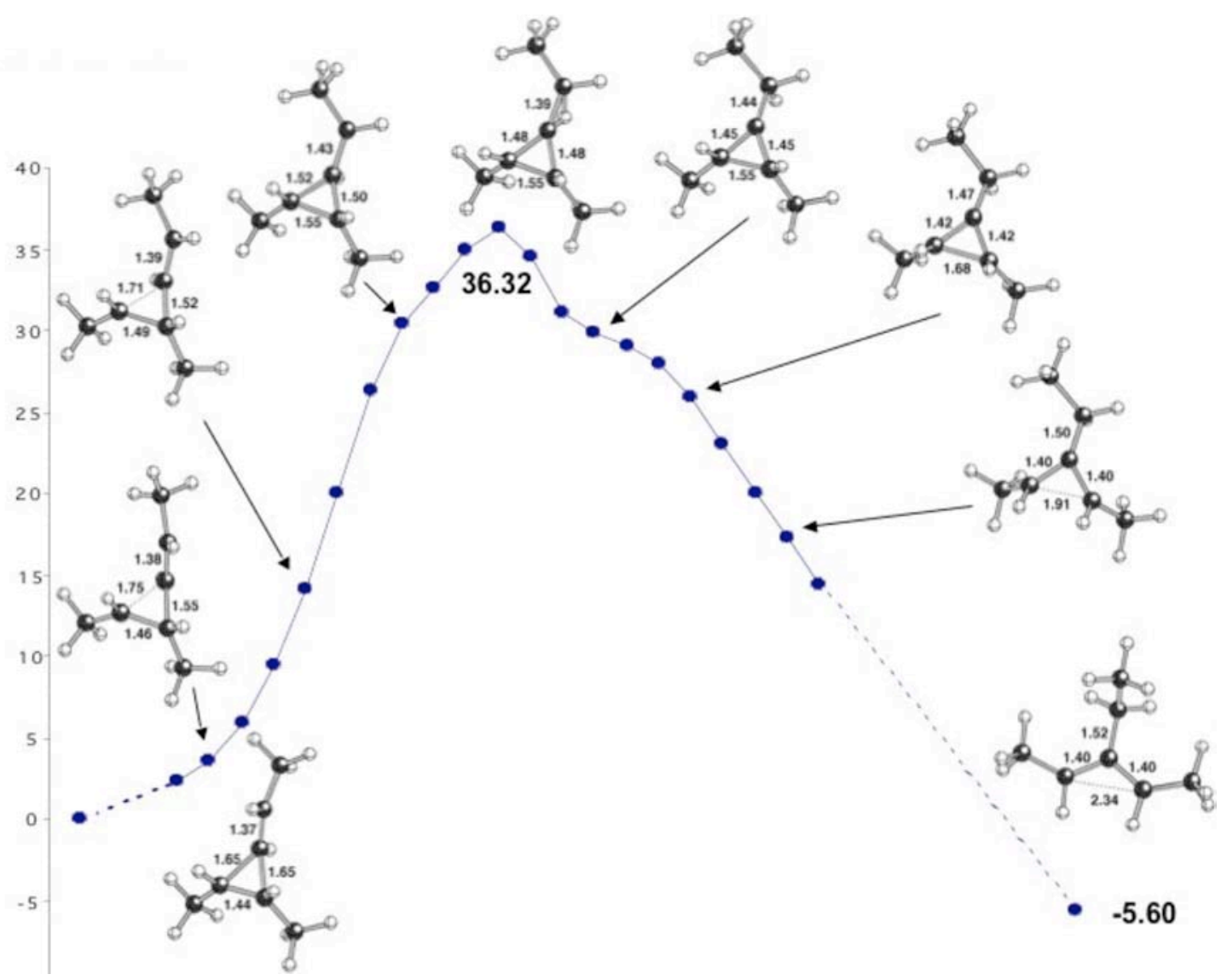




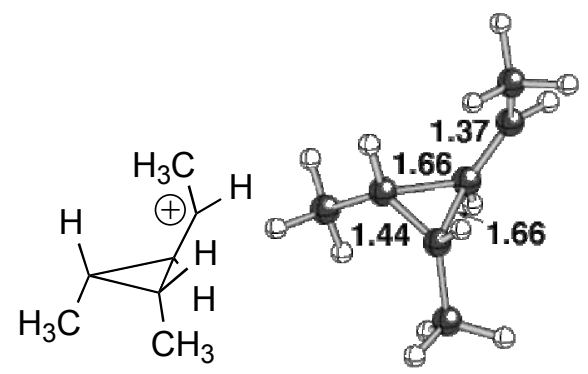

b3lyp/6-31G(d)

\section{Coordinates:}

Standard orientation:

\begin{tabular}{|c|c|c|c|c|c|}
\hline Center & Atomic & & omic & Coordinate & (Angstron \\
\hline Number & Number & & Type & $\begin{array}{ll}X & Y\end{array}$ & Z \\
\hline 1 & 6 & 0 & -0.557867 & -0.717587 & -0.471329 \\
\hline 2 & 6 & 0 & 0.325736 & 0.000302 & 0.732044 \\
\hline 3 & 6 & 0 & -0.557557 & 0.717509 & -0.470973 \\
\hline 4 & 6 & 0 & 1.696236 & -0.000179 & 0.697568 \\
\hline 5 & 6 & 0 & 2.592876 & 0.000026 & -0.477091 \\
\hline 6 & 1 & 0 & 3.260869 & 0.872061 & -0.415402 \\
\hline 7 & 1 & 0 & 2.199696 & -0.001207 & 1.666220 \\
\hline 8 & 1 & 0 & 3.260665 & -0.872189 & -0.415978 \\
\hline 9 & 1 & 0 & 2.088666 & 0.000391 & -1.445020 \\
\hline 10 & 6 & 0 & -1.673029 & -1.597979 & 0.033463 \\
\hline 11 & 1 & 0 & -2.331865 & -1.824019 & -0.814915 \\
\hline 12 & 1 & 0 & -1.281124 & -2.545471 & 0.412570 \\
\hline 13 & 1 & 0 & -2.277214 & -1.126427 & 0.811298 \\
\hline 14 & 6 & 0 & -1.672851 & 1.598111 & 0.033406 \\
\hline 15 & 1 & 0 & -1.280858 & 2.545790 & 0.411936 \\
\hline 16 & 1 & 0 & -2.332045 & 1.823559 & -0.814810 \\
\hline 17 & 1 & 0 & -2.276652 & 1.126904 & 0.811761 \\
\hline 18 & 1 & 0 & 0.114203 & -1.195485 & -1.180768 \\
\hline 19 & 1 & 0 & 0.114521 & 1.195092 & -1.180617 \\
\hline 20 & 1 & 0 & -0.180118 & -0.000212 & 1.691194 \\
\hline
\end{tabular}

Energy: $\mathrm{HF}=-274.2789144$

Zero-Point Energy Correction: 0.182451

Imaginary Frequency: Minimum (No imaginary frequencies) 


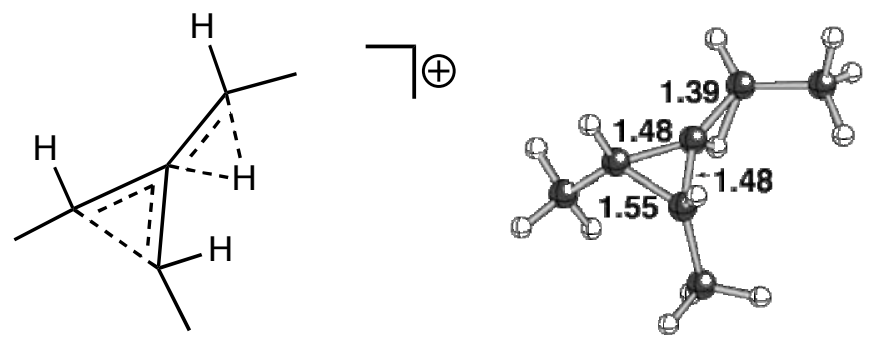

b3lyp/6-31G(d)

\section{Coordinates:}

Standard orientation:

\begin{tabular}{|c|c|c|c|c|}
\hline \multirow{2}{*}{$\begin{array}{l}\text { Center } \\
\text { Number }\end{array}$} & \multirow{2}{*}{$\begin{array}{l}\text { Atomic } \\
\text { Number }\end{array}$} & \multirow{2}{*}{$\begin{array}{r}\text { Atomic } \\
\text { Type }\end{array}$} & \multicolumn{2}{|c|}{ Coordinates (Angstrol } \\
\hline & & & X $\quad Y$ & $\mathrm{Z}$ \\
\hline 1 & 6 & 1.044999 & -0.689106 & 0.564650 \\
\hline 2 & 6 & -0.333873 & -0.326289 & 0.164965 \\
\hline 3 & 6 & 0.546686 & 0.775003 & 0.612696 \\
\hline 4 & 6 & -1.629080 & -0.753763 & -0.085089 \\
\hline 5 & 6 & -2.830581 & 0.124042 & -0.146878 \\
\hline 6 & 1 & -3.437879 & -0.082882 & -1.033736 \\
\hline 7 & 1 & -1.772265 & -1.826034 & -0.248811 \\
\hline 8 & 1 & -0.663771 & -0.429534 & -1.047843 \\
\hline 9 & 1 & -3.449084 & -0.147677 & 0.722669 \\
\hline 10 & 1 & -2.584235 & 1.185351 & -0.082250 \\
\hline 11 & 6 & 2.097033 & -1.173431 & -0.420189 \\
\hline 12 & 1 & 3.093945 & -0.965014 & -0.021399 \\
\hline 13 & 1 & 2.010806 & -2.254784 & -0.564864 \\
\hline 14 & 1 & 2.014790 & -0.690199 & -1.397801 \\
\hline 15 & 6 & 1.070962 & 1.852743 & -0.324247 \\
\hline 16 & 1 & 0.348859 & 2.671081 & -0.404883 \\
\hline 17 & 1 & 2.002063 & 2.263441 & 0.076731 \\
\hline 18 & 1 & 1.272875 & 1.475468 & -1.330527 \\
\hline 19 & 1 & 1.078177 & -1.197057 & 1.529961 \\
\hline 20 & 1 & 0.288833 & 1.142644 & 1.607305 \\
\hline
\end{tabular}

Energy: $\mathrm{HF}=-274.2236127$

Zero-Point Energy Correction: $\mathbf{0 . 1 7 8 7 6 3}$

Imaginary Frequency: One imaginary frequency $-424.9187 \mathrm{~cm}^{-1}$ 

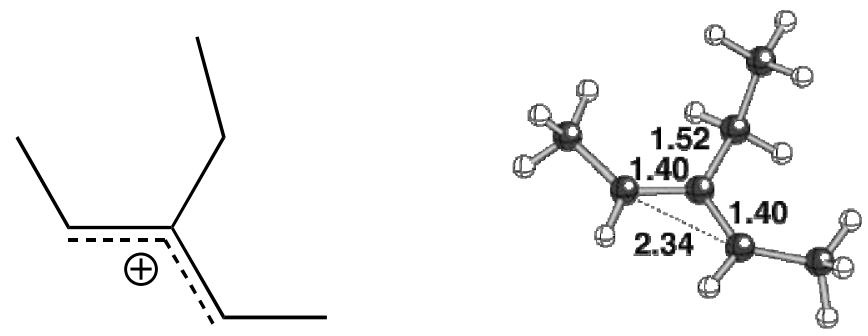

b31yp/6-31G(d)

Coordinates:

Standard orientation:

\begin{tabular}{|c|c|c|c|c|c|}
\hline Center & Atomic & & tomic & Coordinate & es (Angstrom \\
\hline Number & & & Type & $\begin{array}{ll}X & Y\end{array}$ & Z \\
\hline 1 & 6 & 0 & 1.152506 & -1.063064 & -0.132582 \\
\hline 2 & 6 & 0 & -0.009662 & -0.338371 & 0.144059 \\
\hline 3 & 6 & 0 & -1.187012 & -1.037349 & -0.134035 \\
\hline 4 & 6 & 0 & 0.007841 & 1.087841 & 0.656961 \\
\hline 5 & 6 & 0 & 0.051889 & 2.131149 & -0.476449 \\
\hline 6 & 1 & 0 & 0.062301 & 3.139146 & -0.052769 \\
\hline 7 & 1 & 0 & 0.869021 & 1.220757 & 1.319132 \\
\hline 8 & 1 & 0 & 0.947914 & 2.016933 & -1.095738 \\
\hline 9 & 1 & 0 & -0.820878 & 2.050683 & -1.133210 \\
\hline 10 & 6 & 0 & 2.545195 & -0.619227 & 0.013030 \\
\hline 11 & 1 & 0 & 3.061204 & -1.299376 & 0.711493 \\
\hline 12 & 1 & 0 & 3.070979 & -0.767744 & -0.943648 \\
\hline 13 & 1 & 0 & 2.666820 & 0.410654 & 0.346745 \\
\hline 14 & 6 & 0 & -2.570444 & -0.563939 & 0.006687 \\
\hline 15 & 1 & 0 & -3.099823 & -0.712931 & -0.947736 \\
\hline 16 & 1 & 0 & -3.099149 & -1.225699 & 0.713502 \\
\hline 17 & 1 & 0 & -2.671472 & 0.471567 & 0.329646 \\
\hline 18 & 1 & 0 & 1.026465 & -2.082864 & -0.498625 \\
\hline 19 & 1 & 0 & -1.082158 & -2.060221 & -0.498238 \\
\hline 20 & 1 & 0 & -0.873115 & 1.256859 & 1.283425 \\
\hline
\end{tabular}

Energy: $\mathrm{HF}=-274.2904173$

Zero-Point Energy Correction: 0.182078

Imaginary Frequency: Minimum (No imaginary frequencies) 


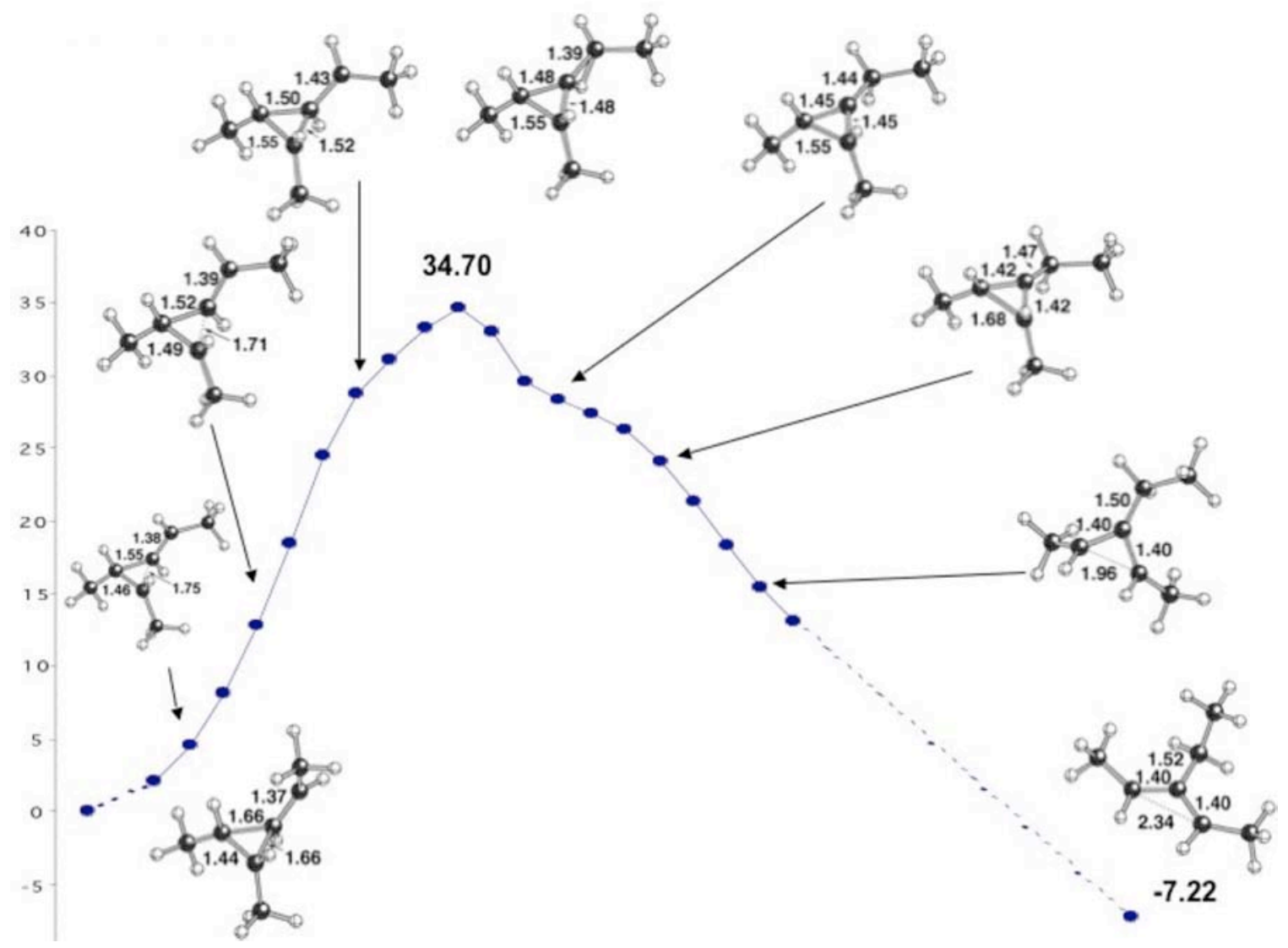

S113 


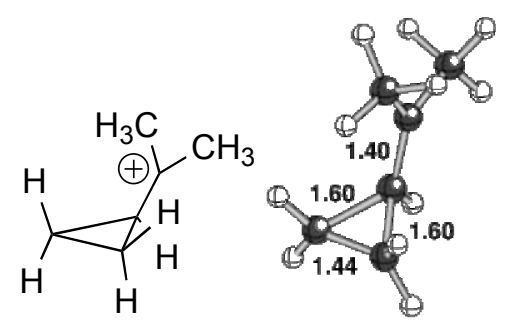

b31yp/6-31G(d)

\section{Coordinates:}

Standard orientation:

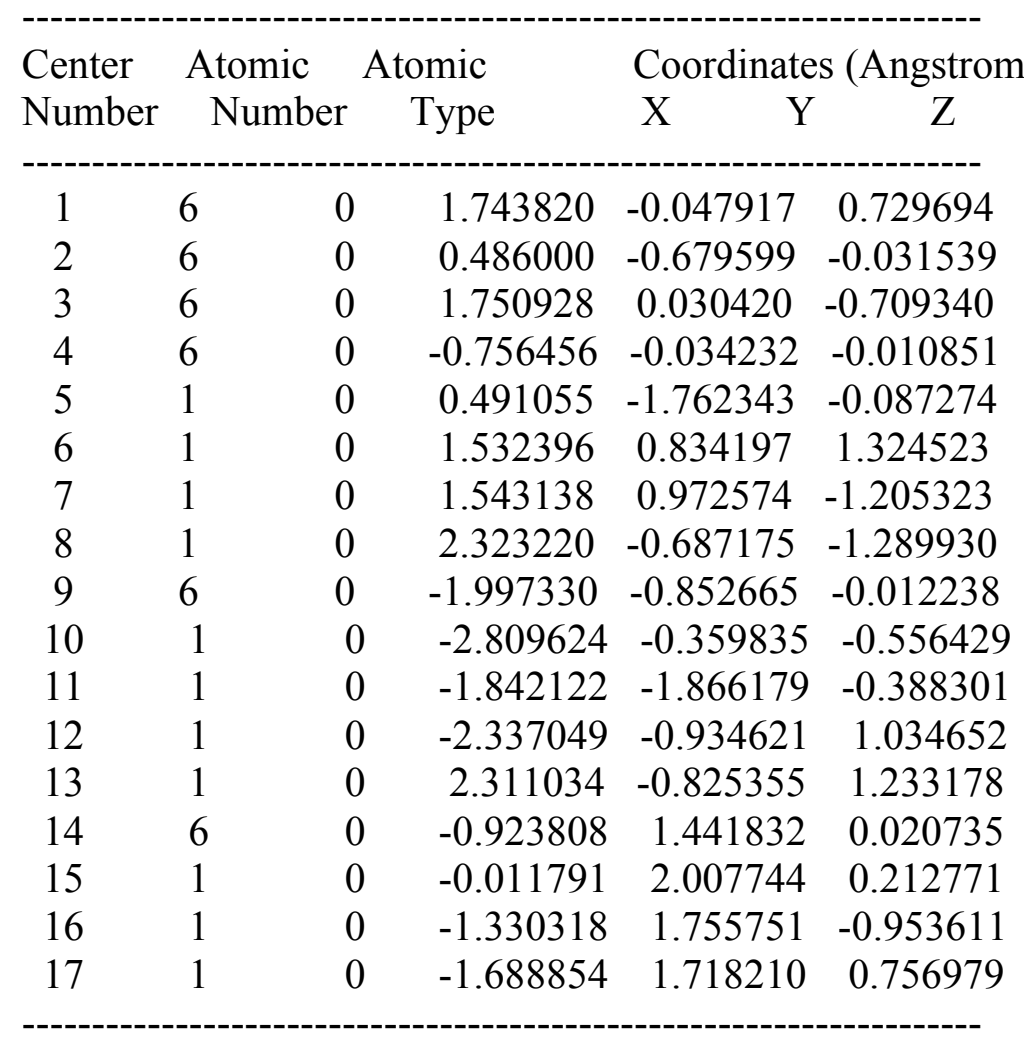

Energy: $\mathrm{HF}=-234.9650199$

Zero-Point Energy Correction: 0.154275

Imaginary Frequency: Minimum (No imaginary frequencies) 
<smiles>CC1(C)CC1</smiles>

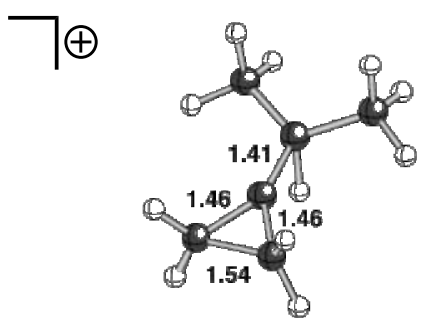

b3lyp/6-31G(d)

\section{Coordinates:}

Standard orientation:

\begin{tabular}{|c|c|c|c|c|}
\hline \multirow{2}{*}{$\begin{array}{l}\text { Center } \\
\text { Number }\end{array}$} & \multirow{2}{*}{$\begin{array}{l}\text { Atomic } \\
\text { Number }\end{array}$} & \multirow{2}{*}{$\begin{array}{r}\text { Atomic } \\
\text { Type }\end{array}$} & \multicolumn{2}{|c|}{ Coordinates (Angstrom } \\
\hline & & & $\begin{array}{ll}X & Y\end{array}$ & I $\quad \mathrm{Z}$ \\
\hline 1 & 6 & 1.843565 & -0.770744 & 0.047662 \\
\hline 2 & 6 & 0.612820 & -0.000042 & -0.027756 \\
\hline 3 & 6 & 1.843534 & 0.770715 & 0.047535 \\
\hline 4 & 6 & -0.788247 & -0.000004 & -0.142944 \\
\hline 5 & 1 & -0.364751 & -0.000099 & -1.259954 \\
\hline 6 & 1 & 2.010685 & -1.336014 & 0.965725 \\
\hline 7 & 1 & 2.010653 & 1.336087 & 0.965534 \\
\hline 8 & 1 & 2.224009 & 1.259527 & -0.848911 \\
\hline 9 & 6 & -1.553435 & -1.303003 & 0.059985 \\
\hline 10 & 1 & -2.452199 & -1.318322 & -0.561307 \\
\hline 11 & 1 & -0.944456 & -2.181552 & -0.166303 \\
\hline 12 & 1 & -1.870722 & -1.358011 & 1.107088 \\
\hline 13 & 1 & 2.224062 & -1.259644 & -0.848725 \\
\hline 14 & 6 & -1.553305 & 1.303063 & 0.060040 \\
\hline 15 & 1 & -1.870455 & 1.358105 & 1.107182 \\
\hline 16 & 1 & -0.944275 & 2.181556 & -0.166324 \\
\hline 17 & 1 & -2.452140 & 1.318452 & -0.561146 \\
\hline
\end{tabular}

Energy: $\mathrm{HF}=-234.9105983$

Zero-Point Energy Correction: 0.149996

Imaginary Frequency: One imaginary frequency $-315.3546 \mathrm{~cm}^{-1}$ 

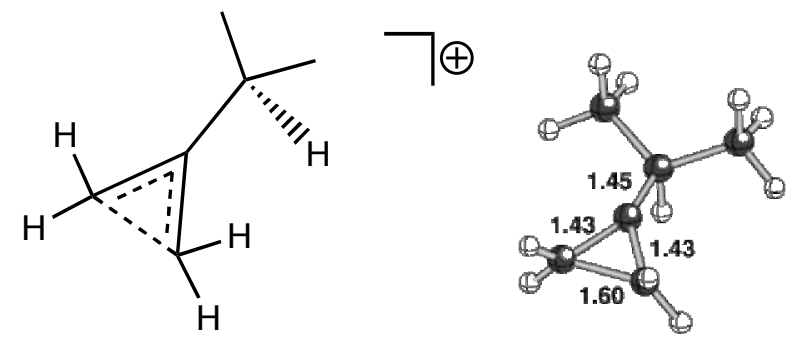

b3lyp/6-31G(d)

\section{Coordinates:}

Standard orientation:

\begin{tabular}{|c|c|c|c|c|}
\hline \multirow{2}{*}{$\begin{array}{l}\text { Center } \\
\text { Number }\end{array}$} & \multirow{2}{*}{$\begin{array}{l}\text { Atomic } \\
\text { Number }\end{array}$} & \multirow{2}{*}{$\begin{array}{r}\text { Atomic } \\
\text { Type }\end{array}$} & \multicolumn{2}{|c|}{ Coordinates (Angstron } \\
\hline & & & X $\quad Y$ & $\mathrm{Z}$ \\
\hline 1 & 6 & -1.806571 & 0.800070 & 0.008614 \\
\hline 2 & 6 & -0.626282 & -0.001161 & 0.087800 \\
\hline 3 & 6 & -1.808319 & -0.798675 & -0.003545 \\
\hline 4 & 6 & 0.778170 & -0.000501 & -0.266823 \\
\hline 5 & 1 & 0.646347 & -0.005678 & -1.381777 \\
\hline 6 & 1 & -2.313610 & 1.144432 & 0.909184 \\
\hline 7 & 1 & -2.315890 & -1.155530 & 0.891831 \\
\hline 8 & 1 & -1.912368 & -1.457539 & -0.867931 \\
\hline 9 & 6 & 1.522413 & 1.301366 & 0.095597 \\
\hline 10 & 1 & 2.495523 & 1.310082 & -0.400579 \\
\hline 11 & 1 & 0.968380 & 2.191401 & -0.215375 \\
\hline 12 & 1 & 1.692019 & 1.350344 & 1.175329 \\
\hline 13 & 1 & -1.908745 & 1.472432 & -0.845571 \\
\hline 14 & 6 & 1.522632 & -1.301289 & 0.103053 \\
\hline 15 & 1 & 1.691613 & -1.344088 & 1.183106 \\
\hline 16 & 1 & 0.968661 & -2.192842 & -0.203527 \\
\hline 17 & 1 & 2.495812 & -1.311881 & -0.392871 \\
\hline
\end{tabular}

Energy: $\mathrm{HF}=-234.9095256$

Zero-Point Energy Correction: $\mathbf{0 . 1 5 0 8 8 9}$

Imaginary Frequency: One imaginary frequency $-251.1122 \mathrm{~cm}^{-1}$ 

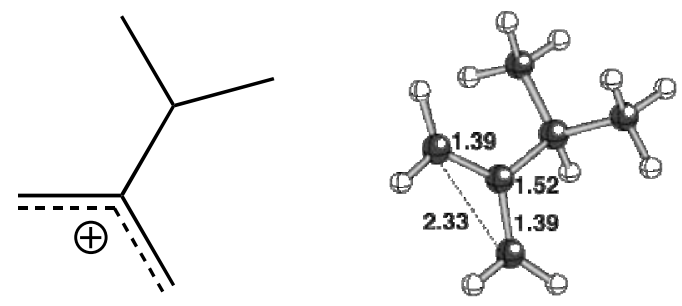

b3lyp/6-31G(d)

\section{Coordinates:}

Standard orientation:

\begin{tabular}{|c|c|c|c|c|c|}
\hline \multirow{2}{*}{$\begin{array}{l}\text { Center } \\
\text { Number }\end{array}$} & \multirow{2}{*}{$\begin{array}{l}\text { Atomic } \\
\text { Number }\end{array}$} & \multirow{2}{*}{\multicolumn{2}{|c|}{$\begin{array}{l}\text { Atomic } \\
\text { Type }\end{array}$}} & \multicolumn{2}{|c|}{ Coordinates (Angstro } \\
\hline & & & & $\begin{array}{ll}X & Y\end{array}$ & $\mathrm{Z}$ \\
\hline 1 & 6 & 0 & -1.274045 & 1.195490 & 0.574564 \\
\hline 2 & 6 & 0 & -0.798526 & 0.059274 & -0.071180 \\
\hline 3 & 6 & 0 & -1.794053 & -0.838487 & -0.441305 \\
\hline 4 & 6 & 0 & 0.670052 & -0.211607 & -0.374570 \\
\hline 5 & 1 & 0 & 0.696043 & -0.668087 & -1.373171 \\
\hline 6 & 1 & 0 & -2.331531 & 1.320525 & 0.798042 \\
\hline 7 & 1 & 0 & -2.8 & -0.660036 & 13324 \\
\hline 8 & 1 & 0 & -1.562760 & -1.750343 & -0.990802 \\
\hline 9 & 6 & 0 & 1.525383 & 1.062039 & -0.407049 \\
\hline 10 & 1 & 0 & 2.534 & 0.810954 & -0.745306 \\
\hline 11 & 1 & 0 & 1.125041 & 1.813572 & -1.097294 \\
\hline 12 & 1 & 0 & & 1.513785 & 0.587316 \\
\hline 13 & 1 & 0 & -0.613785 & 2.006985 & 0.871590 \\
\hline 14 & 6 & 0 & 1.217026 & -1.245408 & 0.635692 \\
\hline 15 & 1 & 0 & 1.209239 & -0.843290 & 1.655203 \\
\hline 16 & 1 & 0 & 0.636378 & -2.175263 & 0.632570 \\
\hline 17 & 1 & 0 & 2.249708 & -1.496603 & 0.378263 \\
\hline
\end{tabular}

Energy: $\mathrm{HF}=-234.9288183$

Zero-Point Energy Correction: 0.153846

Imaginary Frequency: Minimum (No imaginary frequencies) 
Constructed from two IRCs from two different transitition states (One at 34.15, the other at $34.82 \mathrm{kcal} / \mathrm{mol}$ )

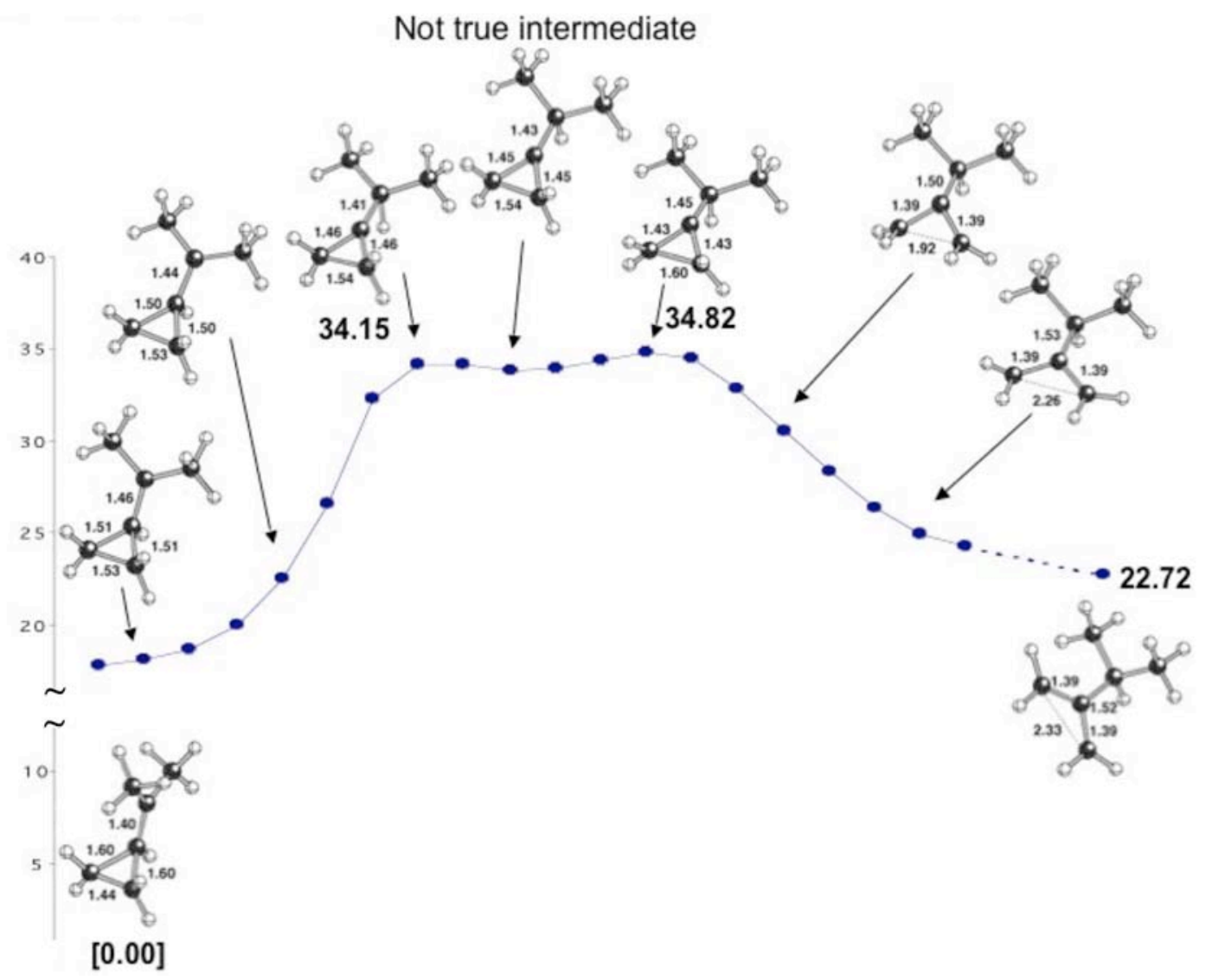



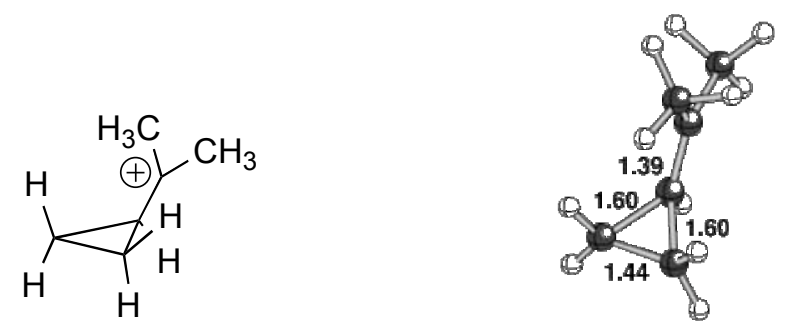

MP2/6-31G(d)

Coordinates:

Standard orientation:

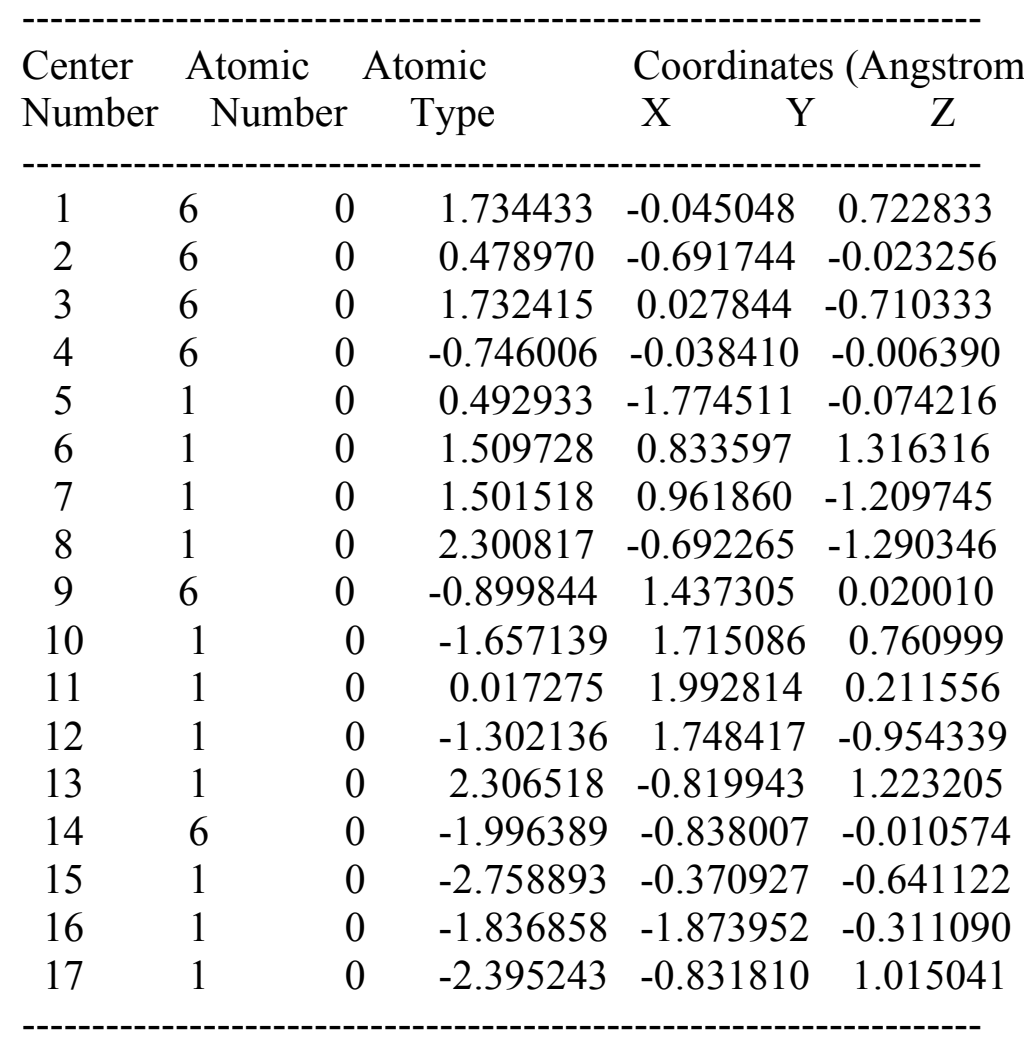

Energy: MP2 $=-234.0956086$

Zero-Point Energy Correction: 0.157521

Imaginary Frequency: Minimum (No imaginary frequencies) 
<smiles>CC1(C)C2C=CC21</smiles>

MP2/6-31G(d)

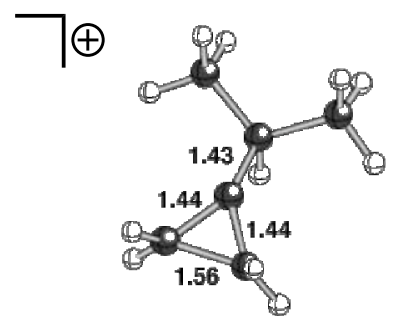

\section{Coordinates:}

Standard orientation:

\begin{tabular}{|c|c|c|c|c|c|}
\hline \multirow{2}{*}{$\begin{array}{l}\text { Center } \\
\text { Number }\end{array}$} & \multirow{2}{*}{\multicolumn{2}{|c|}{$\begin{array}{l}\text { Atomic } \\
\text { Number }\end{array}$}} & \multirow{2}{*}{$\begin{array}{l}\text { Atomic } \\
\text { Type }\end{array}$} & \multicolumn{2}{|c|}{ Coordinates (Angstrom } \\
\hline & & & & $\begin{array}{ll}X & Y\end{array}$ & $\mathrm{Z}$ \\
\hline 1 & 6 & 0 & 1.816018 & -0.782277 & 0.006883 \\
\hline 2 & 6 & 0 & 0.614537 & -0.000214 & 0.075850 \\
\hline 3 & 6 & 0 & 1.815743 & 0.782388 & 0.008619 \\
\hline 4 & 6 & 0 & -0.779428 & -0.000071 & -0.242938 \\
\hline 5 & 1 & 0 & -0.579975 & -0.000956 & -1.357635 \\
\hline 6 & 1 & 0 & 2.242721 & -1.182558 & 0.926504 \\
\hline 7 & 1 & 0 & 2.242435 & 1.180949 & 0.928990 \\
\hline 8 & 1 & 0 & 1.957207 & 1.418072 & -0.866209 \\
\hline 9 & 6 & 0 & -1.525131 & -1.295652 & 0.091228 \\
\hline 10 & 1 & 0 & -2.481484 & -1.308447 & -0.434184 \\
\hline 11 & 1 & 0 & -0.954745 & -2.180592 & -0.198896 \\
\hline 12 & 1 & 0 & -1.725025 & -1.336271 & 1.164362 \\
\hline 13 & 1 & 0 & 1.957825 & -1.416116 & -0.869220 \\
\hline 14 & 6 & 0 & -1.524851 & 1.295778 & 0.090169 \\
\hline 15 & 1 & 0 & -1.724063 & 1.337753 & 1.163384 \\
\hline 16 & 1 & 0 & -0.954648 & 2.180375 & -0.201364 \\
\hline 17 & 1 & 0 & -2.481571 & 1.308077 & -0.434597 \\
\hline
\end{tabular}

Energy: MP2 $=-234.0361388$

Zero-Point Energy Correction: 0.153735

Imaginary Frequency: One imaginary frequency $-331.3053 \mathrm{~cm}^{-1}$ 
$\mathrm{MP} 2 / 6-31 \mathrm{G}(\mathrm{d})$
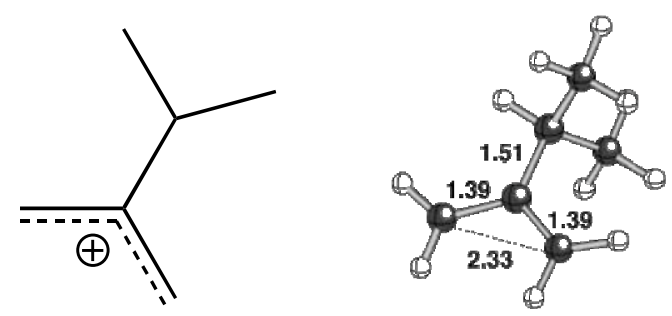

Coordinates:

Standard orientation:

\begin{tabular}{cccccc} 
Center & \multicolumn{2}{c}{ Atomic } & \multicolumn{2}{c}{ Atomic } & \multicolumn{3}{c}{ Coordinates } \\
Number & Number & Type & X & Y & Z \\
- & Nutroms) \\
\hline 1 & 6 & 0 & 1.865669 & 0.017980 & -0.913170 \\
2 & 6 & 0 & 0.791763 & 0.000419 & -0.030712 \\
3 & 6 & 0 & 1.168000 & -0.025736 & 1.308378 \\
4 & 6 & 0 & -0.655260 & 0.008634 & -0.470100 \\
5 & 1 & 0 & -0.657509 & 0.028442 & -1.566349 \\
6 & 1 & 0 & 2.894529 & 0.010688 & -0.557412 \\
7 & 1 & 0 & 2.215872 & -0.030856 & 1.602656 \\
8 & 1 & 0 & 0.435751 & -0.041368 & 2.112929 \\
9 & 6 & 0 & -1.360297 & -1.270752 & -0.006013 \\
10 & 1 & 0 & -2.384844 & -1.279622 & -0.385024 \\
11 & 1 & 0 & -0.855889 & -2.168931 & -0.372668 \\
12 & 1 & 0 & -1.413396 & -1.320787 & 1.086468 \\
13 & 1 & 0 & 1.717310 & 0.038588 & -1.991367 \\
14 & 6 & 0 & -1.359803 & 1.270714 & 0.039918 \\
15 & 1 & 0 & -1.411665 & 1.282005 & 1.133555 \\
16 & 1 & 0 & -0.855807 & 2.181327 & -0.295243 \\
17 & 1 & 0 & -2.384782 & 1.292965 & -0.337356
\end{tabular}

Energy: MP2 $=-234.0584954$

Zero-Point Energy Correction: 0.156913

Imaginary Frequency: Minimum (No imaginary frequencies) 


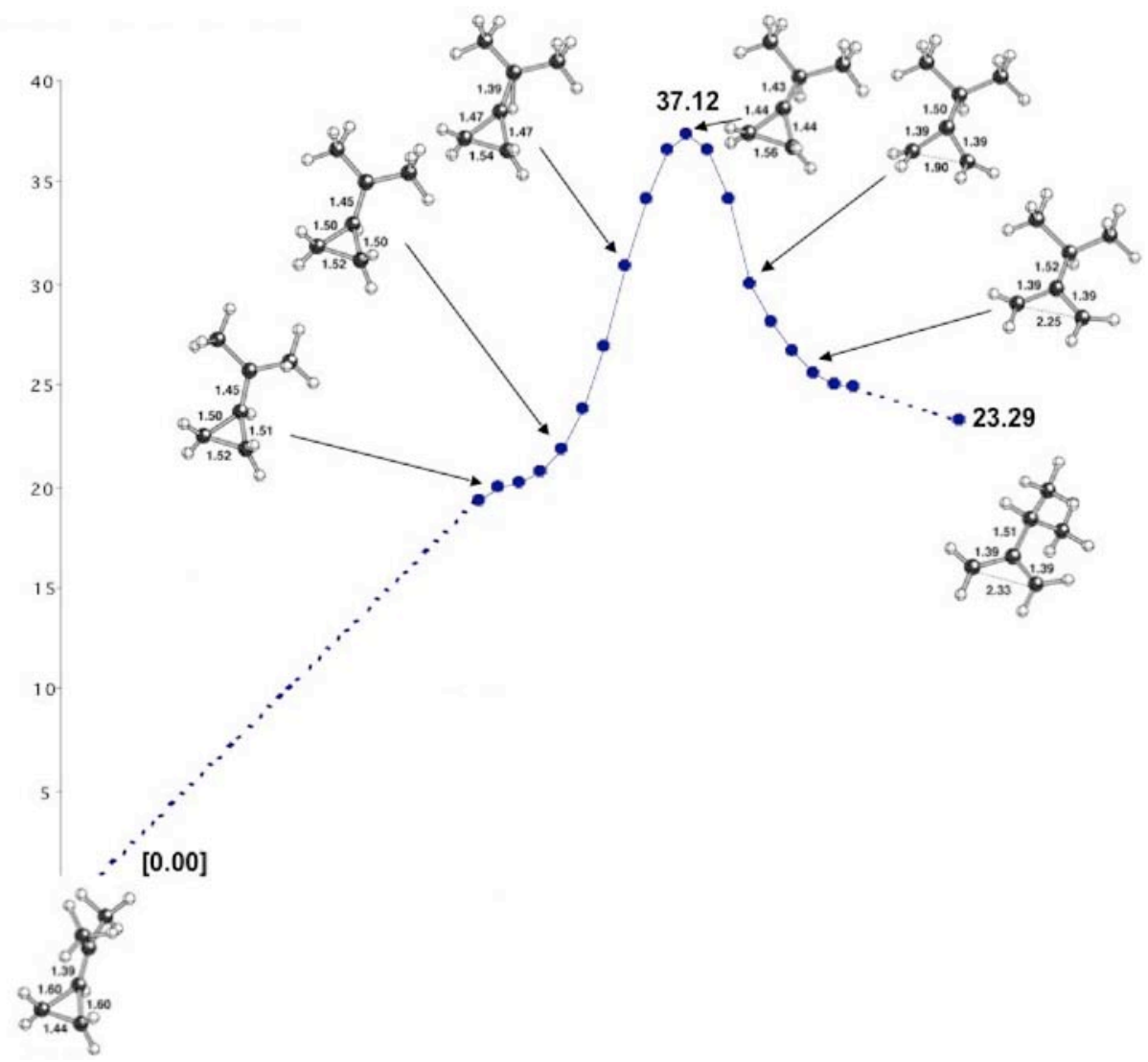

S17? 

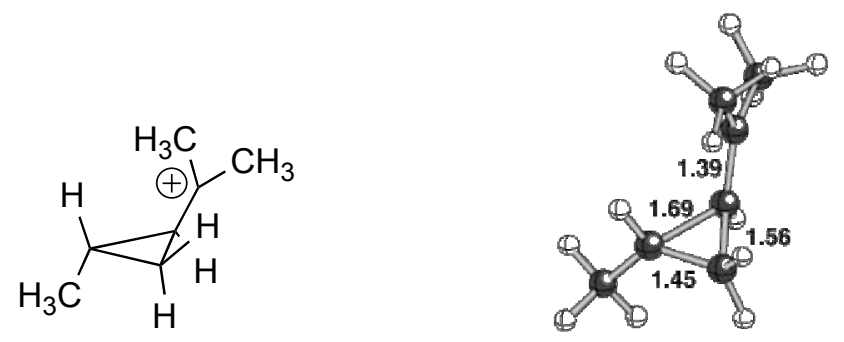

b3lyp/6-31G(d)

\section{Coordinates:}

Standard orientation:

\begin{tabular}{|c|c|c|c|c|c|}
\hline \multirow{2}{*}{$\begin{array}{l}\text { Center } \\
\text { Number }\end{array}$} & \multirow{2}{*}{\multicolumn{2}{|c|}{$\begin{array}{l}\text { Atomic } \\
\text { Number }\end{array}$}} & \multirow{2}{*}{$\begin{array}{l}\text { Atomic } \\
\text { Type }\end{array}$} & \multicolumn{2}{|c|}{ Coordinates (Angstroms) } \\
\hline & & & & X $\quad Y$ & $\mathrm{Z}$ \\
\hline 1 & 6 & 0 & -1.390077 & 0.305175 & -0.327981 \\
\hline 2 & 6 & 0 & -0.099535 & -0.285691 & 0.581851 \\
\hline 3 & 6 & 0 & -1.087407 & 0.860023 & 0.971207 \\
\hline 4 & 6 & 0 & 1.167805 & -0.082400 & 0.044218 \\
\hline 5 & 1 & 0 & -0.318221 & -1.255905 & 1.014353 \\
\hline 6 & 6 & 0 & -2.477937 & -0.698861 & -0.582506 \\
\hline 7 & 1 & 0 & -3.363744 & -0.147030 & -0.925617 \\
\hline 8 & 1 & 0 & -2.204572 & -1.404899 & -1.371765 \\
\hline 9 & 1 & 0 & -2.755720 & -1.250127 & 0.320158 \\
\hline 10 & 1 & 0 & -1.033764 & 0.853212 & -1.196290 \\
\hline 11 & 1 & 0 & -0.649035 & 1.850462 & 1.045461 \\
\hline 12 & 1 & 0 & -1.707945 & 0.571334 & 1.816343 \\
\hline 13 & 6 & 0 & 1.617448 & 1.193851 & -0.579015 \\
\hline 14 & 1 & 0 & 1.815221 & 1.022748 & -1.646927 \\
\hline 15 & 1 & 0 & 0.918593 & 2.025092 & -0.479425 \\
\hline 16 & 1 & 0 & 2.582922 & 1.488110 & -0.146988 \\
\hline 17 & 6 & 0 & 2.164220 & -1.189778 & 0.092253 \\
\hline 18 & 1 & 0 & 2.887141 & -0.961809 & 0.892150 \\
\hline 19 & 1 & 0 & 1.715199 & -2.160307 & 0.312598 \\
\hline 20 & 1 & 0 & 2.746821 & -1.244798 & -0.834211 \\
\hline
\end{tabular}

Energy: $\mathrm{HF}=-274.2888681$

Zero-Point Energy Correction: 0.182410

Imaginary Frequency: Minimum (No imaginary frequencies) 

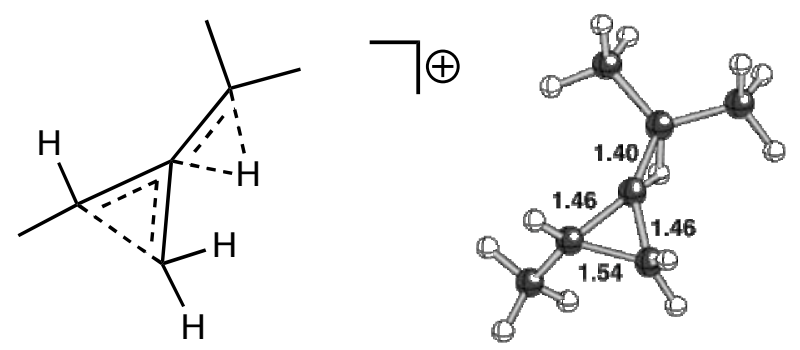

b3lyp/6-31G(d)

\section{Coordinates:}

Standard orientation:

\begin{tabular}{cccccc} 
Center & \multicolumn{2}{c}{ Atomic } & \multicolumn{2}{c}{ Atomic } & \multicolumn{3}{c}{ Coordinates } \\
Number & Number & Type & X & Y & $Z$ \\
- & Numstroms) \\
1 & 6 & 0 & -1.526479 & 0.006524 & 0.491421 \\
2 & 6 & 0 & -0.141985 & -0.320565 & 0.165717 \\
3 & 6 & 0 & -1.078313 & -1.438292 & 0.193347 \\
4 & 6 & 0 & 1.162592 & 0.119537 & -0.104938 \\
5 & 1 & 0 & 0.523675 & 0.144782 & -1.129102 \\
6 & 6 & 0 & -2.453291 & 0.745098 & -0.469592 \\
7 & 1 & 0 & -3.490122 & 0.498921 & -0.225566 \\
8 & 1 & 0 & -2.324072 & 1.827318 & -0.378447 \\
9 & 1 & 0 & -2.267536 & 0.454021 & -1.508779 \\
10 & 1 & 0 & -1.663199 & 0.271161 & 1.543549 \\
11 & 1 & 0 & -0.955122 & -2.168258 & 0.994254 \\
12 & 1 & 0 & -1.403875 & -1.870759 & -0.753338 \\
13 & 6 & 0 & 1.561965 & 1.549938 & 0.230957 \\
14 & 1 & 0 & 2.286749 & 1.926926 & -0.494931 \\
15 & 1 & 0 & 0.703151 & 2.224123 & 0.267691 \\
16 & 1 & 0 & 2.046009 & 1.548883 & 1.213930 \\
17 & 6 & 0 & 2.280496 & -0.899335 & -0.277064 \\
18 & 1 & 0 & 2.798622 & -1.005940 & 0.682487 \\
19 & 1 & 0 & 1.905176 & -1.879674 & -0.579883 \\
20 & 1 & 0 & 3.010637 & -0.548935 & -1.010940
\end{tabular}

Energy: $\mathrm{HF}=-274.2313508$

Zero-Point Energy Correction: 0.178515

Imaginary Frequency: One imaginary frequency $-368.4853 \mathrm{~cm}^{-1}$ 


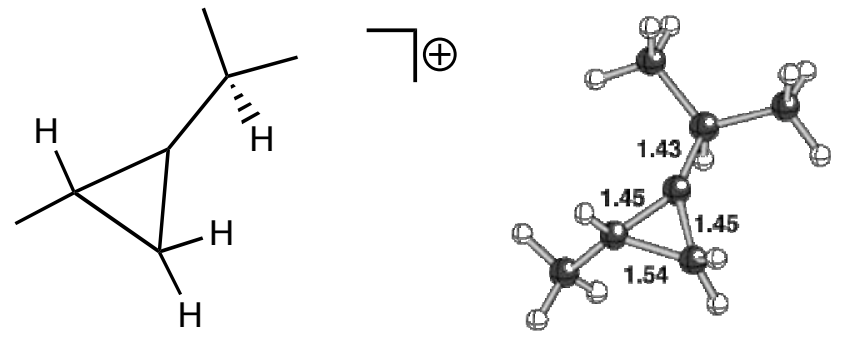

b3lyp/6-31G(d)

\section{Coordinates:}

Standard orientation:

\begin{tabular}{|c|c|c|c|c|c|}
\hline \multirow{2}{*}{$\begin{array}{l}\text { Center } \\
\text { Number }\end{array}$} & \multirow{2}{*}{$\begin{array}{l}\text { Atomic } \\
\text { Number }\end{array}$} & \multirow{2}{*}{\multicolumn{2}{|c|}{$\begin{array}{c}\text { Atomic } \\
\text { Type }\end{array}$}} & \multicolumn{2}{|c|}{ Coordinates (Angstro } \\
\hline & & & & X $\quad Y$ & $\mathrm{Z}$ \\
\hline 1 & 6 & 0 & -1.525604 & -0.019905 & 0.487953 \\
\hline 2 & 6 & 0 & 56 & -0.328873 & 0.143146 \\
\hline 3 & 6 & 0 & -1.060744 & -1.457882 & 0.194106 \\
\hline 4 & 6 & 0 & 1.143865 & 0.147874 & -0.228074 \\
\hline 5 & 1 & 0 & 0.7949 & 0.271524 & -1.305531 \\
\hline 6 & 6 & 0 & $-2.446^{\circ}$ & 0.754644 & -0.458517 \\
\hline 7 & 1 & 0 & -3.4 & 110 & 14468 \\
\hline 8 & 1 & 0 & -2.30 & 1.832205 & -0.3 \\
\hline 9 & 1 & 0 & -2.263555 & 0.484795 & -1.502943 \\
\hline 10 & 1 & 0 & -1.664158 & 0.227141 & 1.546027 \\
\hline 11 & 1 & 0 & -0.9369 & -2.172686 & 1.010966 \\
\hline 12 & 1 & & & -1.9 & \\
\hline 13 & 6 & & 1.529296 & 1.549679 & 0.277543 \\
\hline 14 & 1 & 0 & 2.357042 & 1.940315 & -0.318605 \\
\hline 15 & 1 & 0 & 0.694064 & 2.252556 & 0.219657 \\
\hline 16 & 1 & & & 1.483988 & 1.317708 \\
\hline 17 & 6 & & 2.277 & -0.891807 & -0.240775 \\
\hline 18 & 1 & U & 2.644437 & -1.045251 & 0.778869 \\
\hline 19 & 1 & 0 & 1.948630 & -1.852942 & -0.644788 \\
\hline 20 & 1 & 0 & 3.108551 & -0.523304 & -0.846342 \\
\hline
\end{tabular}

Energy: $\mathrm{HF}=-274.2321909$

Zero-Point Energy Correction: $\mathbf{0 . 1 7 9 0 6 7}$

Imaginary Frequency: Minimum (No imaginary frequencies) 

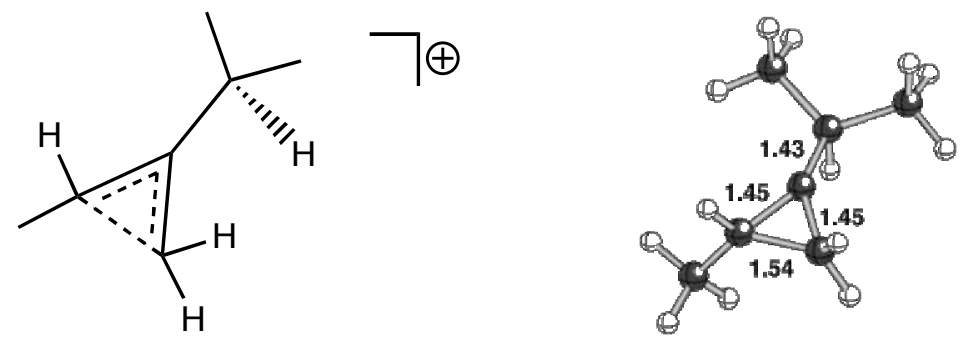

b3lyp/6-31G(d)

Coordinates:

Standard orientation:

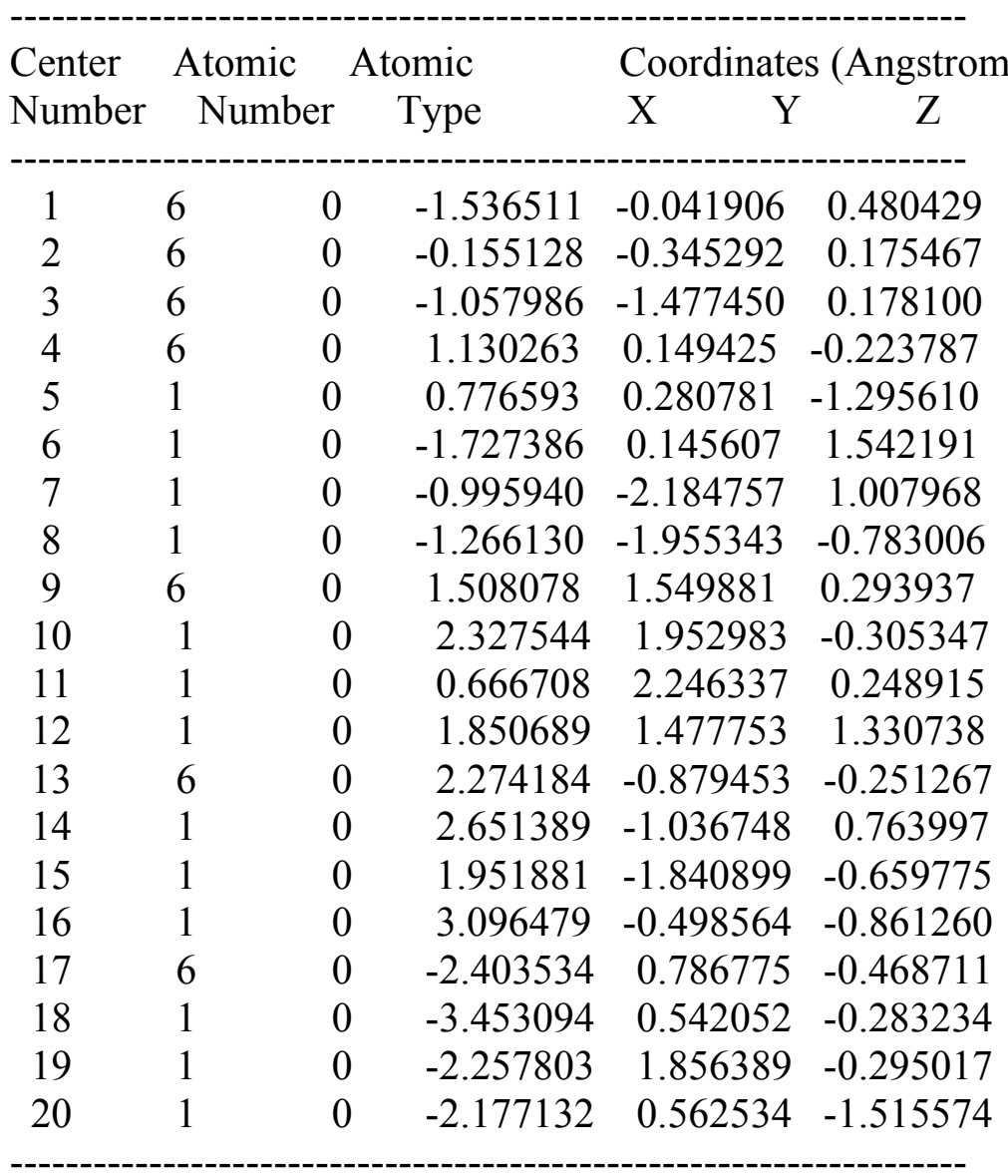

Energy: $\mathrm{HF}=-274.2321859$

Zero-Point Energy Correction: $\mathbf{0 . 1 7 8 9 7 0}$ Imaginary Frequency: One imaginary frequency $-49.1386 \mathrm{~cm}^{-1}$ 
b3lyp/6-31G(d)
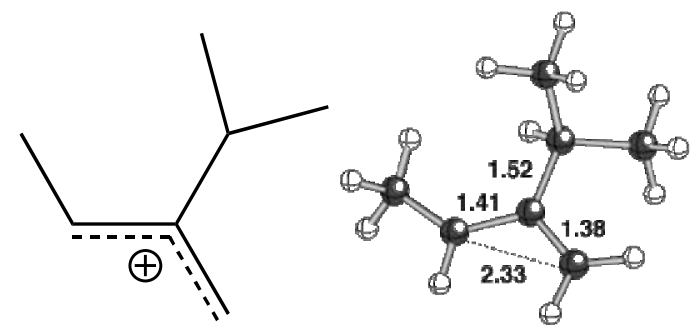

Coordinates:

Standard orientation:

\begin{tabular}{|c|c|c|c|c|c|}
\hline \multirow{2}{*}{$\begin{array}{l}\text { Center } \\
\text { Number }\end{array}$} & \multirow{2}{*}{$\begin{array}{l}\text { Atomic } \\
\text { Number }\end{array}$} & \multirow{2}{*}{\multicolumn{2}{|c|}{$\begin{array}{r}\text { Atomic } \\
\text { Type }\end{array}$}} & \multicolumn{2}{|c|}{ Coordinates (Angstroms) } \\
\hline & & & & X $\quad Y$ & Z \\
\hline 1 & 6 & 0 & 1.623914 & 0.433180 & 0.011412 \\
\hline 2 & 6 & 0 & 0.227496 & 0.633396 & 0.059611 \\
\hline 3 & 6 & 0 & -0.131806 & 1.904837 & 0.453399 \\
\hline 4 & 6 & 0 & -0.776438 & -0.459313 & -0.289725 \\
\hline 5 & 1 & 0 & -0.354071 & -1.019996 & -1.133171 \\
\hline 6 & 1 & 0 & 2.251066 & 1.279694 & 0.295678 \\
\hline 7 & 1 & 0 & 0.612274 & 2.650294 & 0.724058 \\
\hline 8 & 1 & 0 & -1.170329 & 2.219929 & 0.505184 \\
\hline 9 & 6 & 0 & -0.933854 & -1.429215 & 0.903690 \\
\hline 10 & 1 & 0 & -1.607731 & -2.245380 & 0.627681 \\
\hline 11 & 1 & 0 & 0.018394 & -1.873117 & 1.214383 \\
\hline 12 & 1 & 0 & -1.364036 & -0.914348 & 1.769925 \\
\hline 13 & 6 & 0 & -2.136601 & 0.094829 & -0.739476 \\
\hline 14 & 1 & 0 & -2.679950 & 0.570297 & 0.085389 \\
\hline 15 & 1 & 0 & -2.038337 & 0.817040 & -1.557759 \\
\hline 16 & 1 & 0 & -2.760803 & -0.727895 & -1.098742 \\
\hline 17 & 6 & 0 & 2.345834 & -0.772161 & -0.393009 \\
\hline 18 & 1 & 0 & 3.035371 & -0.509891 & -1.214460 \\
\hline 19 & 1 & 0 & 3.025901 & -1.063667 & 0.426577 \\
\hline 20 & 1 & 0 & 1.720987 & -1.616272 & -0.680150 \\
\hline
\end{tabular}

Energy: $\mathrm{HF}=-274.2689147$

Zero-Point Energy Correction: 0.182172

Imaginary Frequency: Minimum (No imaginary frequencies) 
Constructed from two IRCs from two different transition states (One at 36.09, the other at $35.57 \mathrm{kcal} / \mathrm{mol}$ )

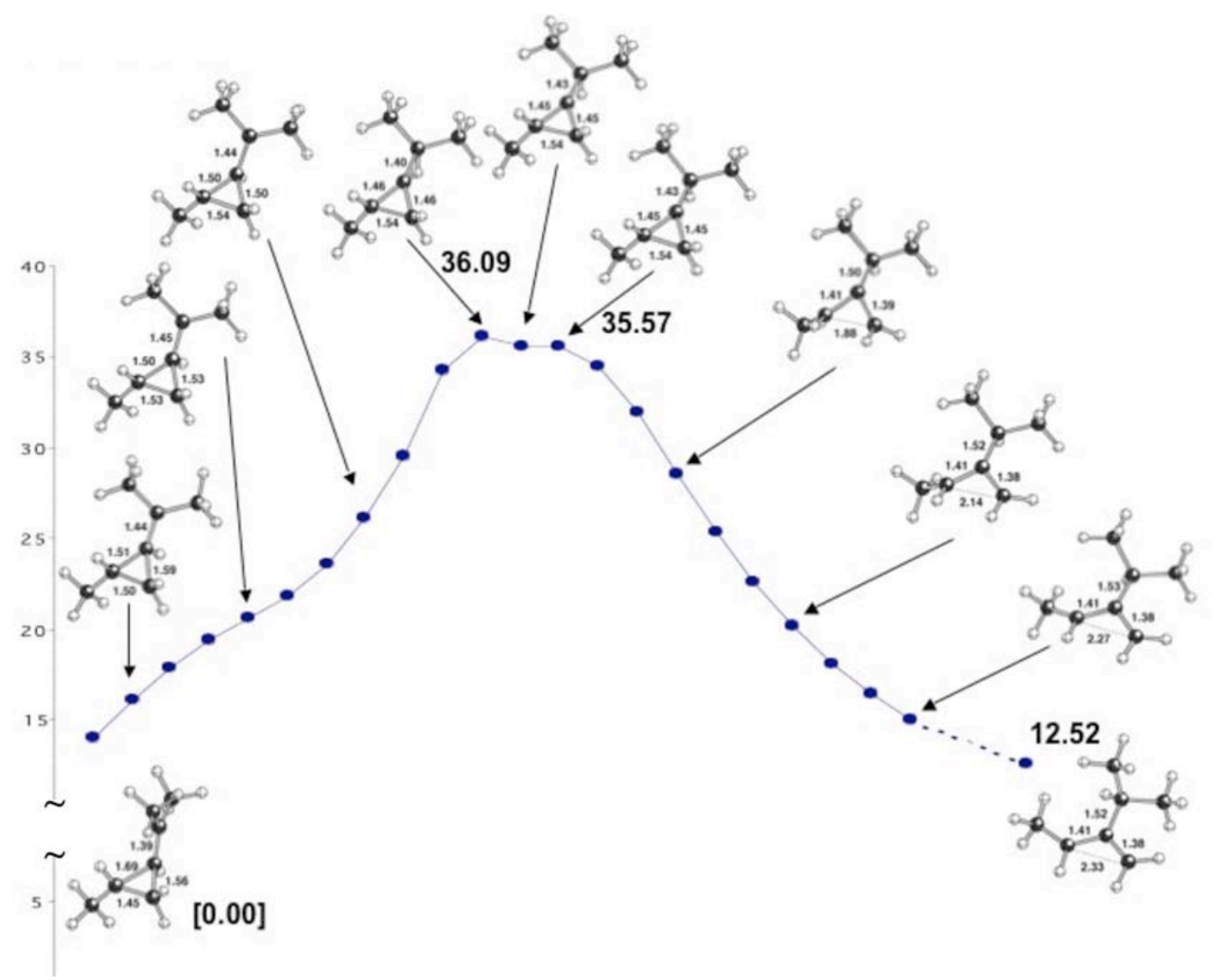


<smiles>CC1C=CCC1C</smiles>

MP2/6-31G(d)

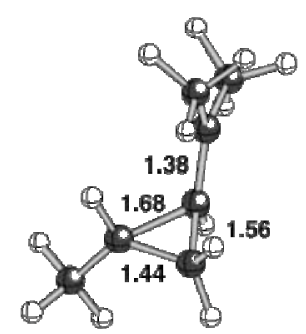

\section{Coordinates:}

Standard orientation:

\begin{tabular}{|c|c|c|c|c|c|}
\hline \multirow{2}{*}{$\begin{array}{l}\text { Center } \\
\text { Number }\end{array}$} & \multirow{2}{*}{\multicolumn{2}{|c|}{$\begin{array}{l}\text { Atomic } \\
\text { Number }\end{array}$}} & \multirow{2}{*}{$\begin{array}{l}\text { Atomic } \\
\text { Type }\end{array}$} & \multicolumn{2}{|c|}{ Coordinates (Angstrom } \\
\hline & & & & X $\quad Y$ & Z \\
\hline 1 & 6 & 0 & -1.356158 & 0.297030 & -0.338778 \\
\hline 2 & 6 & 0 & -0.103870 & -0.271020 & 0.618033 \\
\hline 3 & 6 & 0 & -1.093472 & 0.883290 & 0.950150 \\
\hline 4 & 6 & 0 & 1.145976 & -0.079443 & 0.058086 \\
\hline 5 & 1 & 0 & -0.335210 & -1.231437 & 1.066594 \\
\hline 6 & 6 & 0 & -2.427782 & -0.721272 & -0.586142 \\
\hline 7 & 1 & 0 & -3.305976 & -0.188431 & -0.968788 \\
\hline 8 & 1 & 0 & -2.123968 & -1.452700 & -1.337962 \\
\hline 9 & 1 & 0 & -2.717191 & -1.237639 & 0.331556 \\
\hline 10 & 1 & 0 & -0.974550 & 0.820696 & -1.211693 \\
\hline 11 & 1 & 0 & -0.647352 & 1.870135 & 1.016017 \\
\hline 12 & 1 & 0 & -1.739042 & 0.610629 & 1.781113 \\
\hline 13 & 6 & 0 & 1.586435 & 1.184008 & -0.591966 \\
\hline 14 & 1 & 0 & 1.762272 & 0.994871 & -1.658636 \\
\hline 15 & 1 & 0 & 0.890951 & 2.016116 & -0.488438 \\
\hline 16 & 1 & 0 & 2.556033 & 1.477716 & -0.173814 \\
\hline 17 & 6 & 0 & 2.142522 & -1.183180 & 0.102726 \\
\hline 18 & 1 & 0 & 2.917874 & -0.911780 & 0.832840 \\
\hline 19 & 1 & 0 & 1.705065 & -2.136687 & 0.400588 \\
\hline 20 & 1 & 0 & 2.649189 & -1.287961 & -0.862028 \\
\hline
\end{tabular}

Energy: MP2 $=-273.2724346$

\section{Zero-Point Energy Correction: $\mathbf{0 . 1 8 6 3 5 6}$}

Imaginary Frequency: Minimum (No imaginary frequencies) 

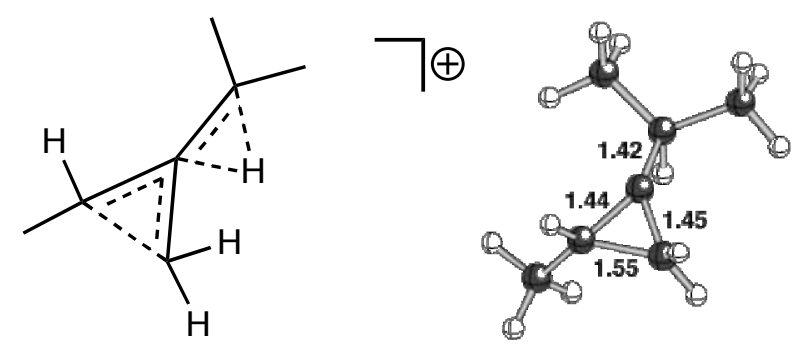

$\mathrm{MP} 2 / 6-31 \mathrm{G}(\mathrm{d})$

Coordinates:

Standard orientation:

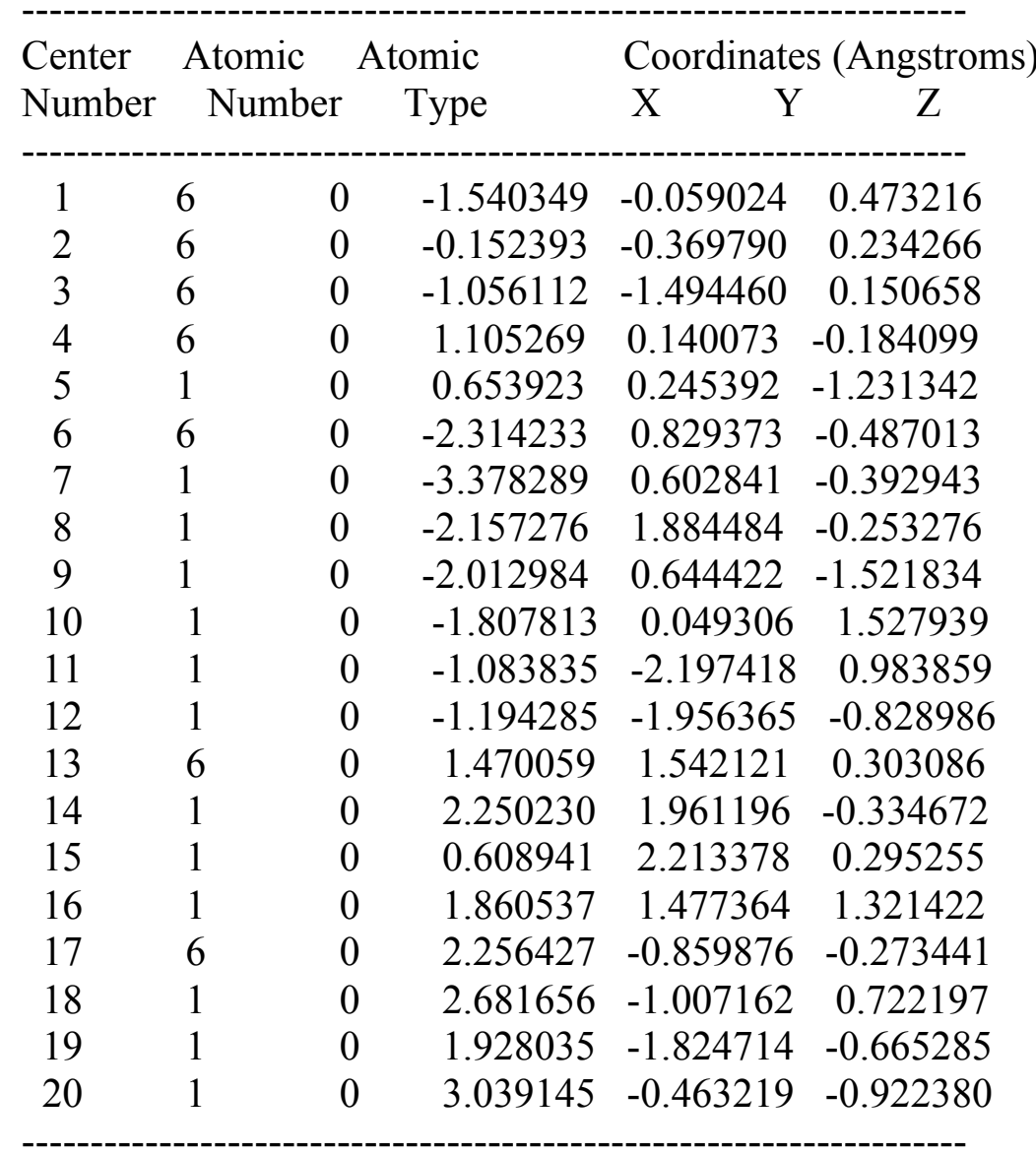

Energy: MP2 $=-273.2111018$

Zero-Point Energy Correction: $\mathbf{0 . 1 8 2 5 8 0}$

Imaginary Frequency: One imaginary frequency $-225.2970 \mathrm{~cm}^{-1}$ 

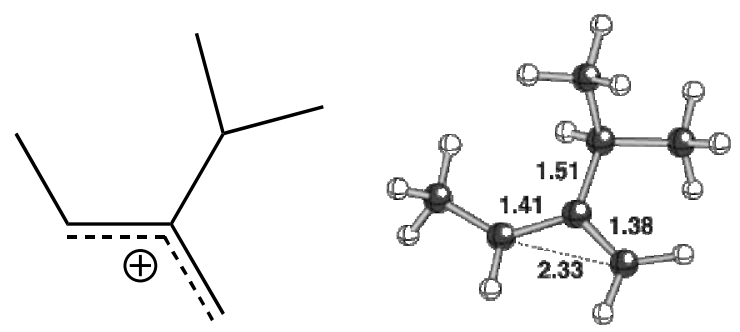

MP2/6-31G(d)

Coordinates:

Standard orientation:

\begin{tabular}{|c|c|c|c|c|}
\hline \multirow{2}{*}{$\begin{array}{l}\text { Center } \\
\text { Number }\end{array}$} & Atomic & \multirow{2}{*}{$\begin{array}{l}\text { Atomic } \\
\text { Type }\end{array}$} & \multicolumn{2}{|c|}{ Coordinates (Angstroms) } \\
\hline & Number & & X $\quad Y$ & Z \\
\hline 1 & 6 & 1.612922 & 0.444148 & 0.001777 \\
\hline 2 & 6 & 0.218470 & 0.629190 & 0.041089 \\
\hline 3 & 6 & -0.148745 & 1.896362 & 0.450774 \\
\hline 4 & 6 & -0.768968 & -0.468335 & -0.293646 \\
\hline 5 & 1 & -0.347859 & -1.033361 & -1.133606 \\
\hline 6 & 6 & 2.339391 & -0.765219 & -0.384228 \\
\hline 7 & 1 & 3.029695 & -0.510135 & -1.202845 \\
\hline 8 & 1 & 2.999010 & -1.052386 & 0.449408 \\
\hline 9 & 1 & 1.709069 & -1.604183 & -0.668833 \\
\hline 10 & 1 & 2.233088 & 1.299344 & 0.280015 \\
\hline 11 & 1 & 0.597762 & 2.643091 & 0.712989 \\
\hline 12 & 1 & -1.188123 & 2.203485 & 0.524301 \\
\hline 13 & 6 & -0.911365 & -1.409026 & 0.912439 \\
\hline 14 & 1 & -1.584446 & -2.231991 & 0.660415 \\
\hline 15 & 1 & 0.045771 & -1.838796 & 1.222146 \\
\hline 16 & 1 & -1.336421 & -0.871252 & 1.765371 \\
\hline 17 & 6 & -2.127317 & 0.074584 & -0.737171 \\
\hline 18 & 1 & -2.664028 & 0.546840 & 0.091111 \\
\hline 19 & 1 & -2.033894 & 0.792926 & -1.557119 \\
\hline 20 & 1 & -2.745955 & -0.753805 & -1.089559 \\
\hline
\end{tabular}

Energy: MP2 $=-273.2481648$

Zero-Point Energy Correction: $\mathbf{0 . 1 8 5 8 3 3}$

Imaginary Frequency: Minimum (No imaginary frequencies) 


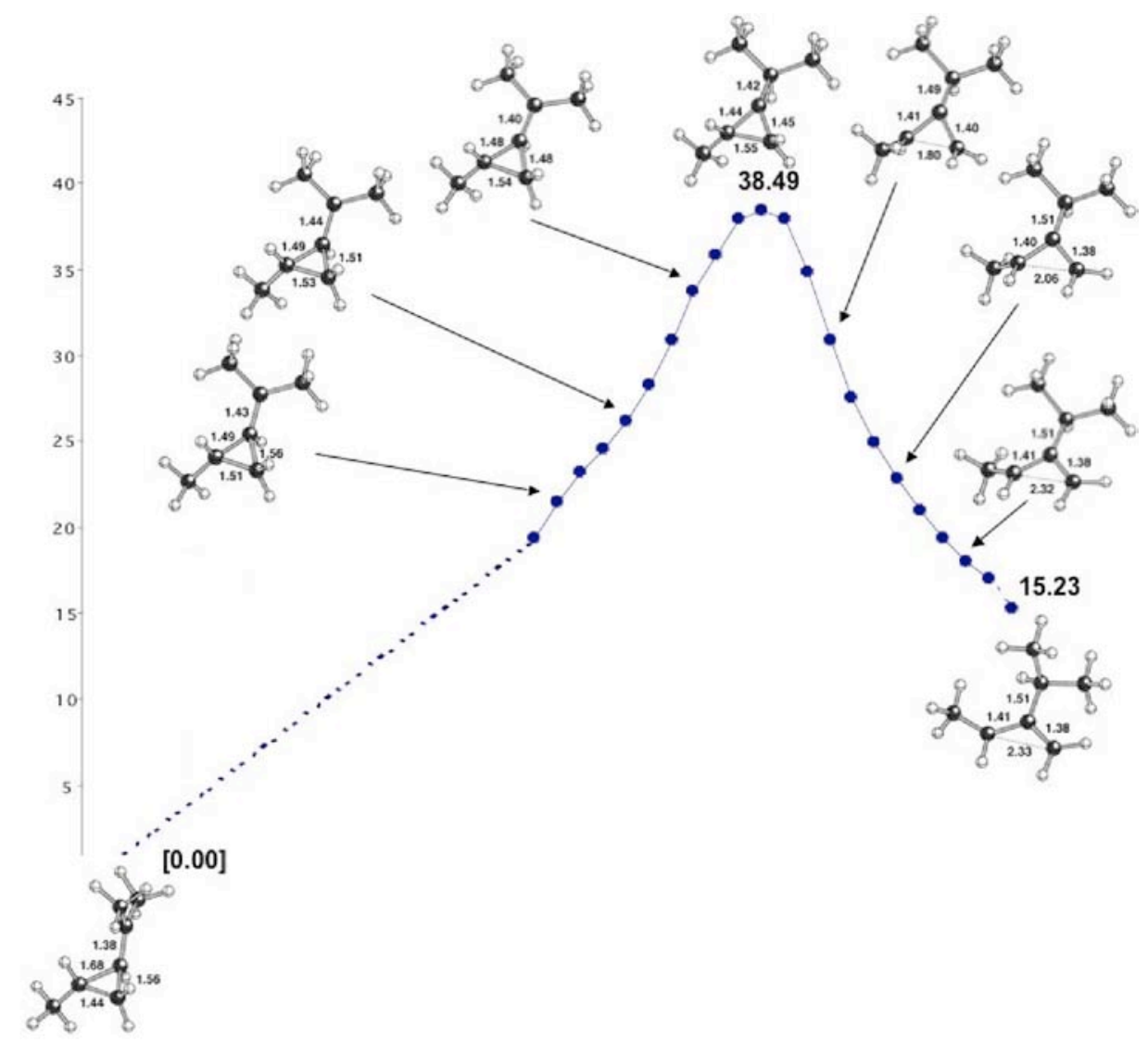

S137 


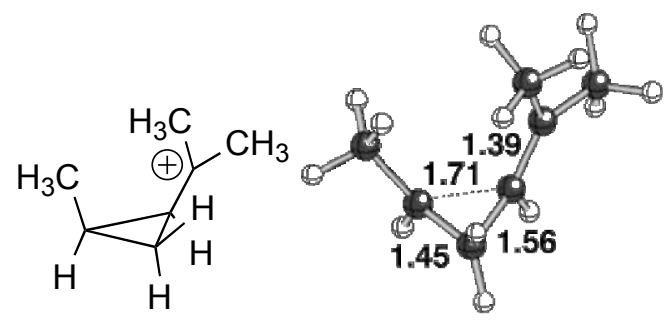

b31yp/6-31G(d)

\section{Coordinates:}

Standard orientation:

\begin{tabular}{|c|c|c|c|c|c|}
\hline \multirow{2}{*}{$\begin{array}{l}\text { Center } \\
\text { Number }\end{array}$} & \multirow{2}{*}{\multicolumn{2}{|c|}{$\begin{array}{l}\text { Atomic } \\
\text { Number }\end{array}$}} & \multirow{2}{*}{$\begin{array}{l}\text { Atomic } \\
\text { Type }\end{array}$} & \multicolumn{2}{|c|}{ Coordinates (Angstroms } \\
\hline & & & & X $\quad Y$ & $Z$ \\
\hline 1 & 6 & 0 & -1.556905 & -0.410899 & -0.357578 \\
\hline 2 & 6 & 0 & 0.025896 & -0.941258 & 0.011322 \\
\hline 3 & 6 & 0 & -1.218674 & -0.920343 & 0.951616 \\
\hline 4 & 6 & 0 & 1.081312 & -0.035193 & 0.050646 \\
\hline 5 & 1 & 0 & 0.213404 & -1.888095 & -0.483324 \\
\hline 6 & 1 & 0 & -1.168577 & -0.221595 & 1.781916 \\
\hline 7 & 1 & 0 & -1.520742 & -1.928495 & 1.225436 \\
\hline 8 & 6 & 0 & 2.358355 & -0.393957 & -0.630420 \\
\hline 9 & 1 & 0 & 3.094723 & -0.667169 & 0.142131 \\
\hline 10 & 1 & 0 & 2.258622 & -1.236112 & -1.317809 \\
\hline 11 & 1 & 0 & 2.781892 & 0.471611 & -1.153864 \\
\hline 12 & 6 & 0 & 1.055214 & 1.282162 & 0.743896 \\
\hline 13 & 1 & 0 & 0.948194 & 2.076377 & -0.011375 \\
\hline 14 & 1 & 0 & 0.257603 & 1.393684 & 1.477938 \\
\hline 15 & 1 & 0 & 2.020235 & 1.464961 & 1.230544 \\
\hline 16 & 6 & 0 & -1.895854 & 1.013580 & -0.682807 \\
\hline 17 & 1 & 0 & -1.525454 & 1.310775 & -1.668380 \\
\hline 18 & 1 & 0 & -2.992527 & 1.068707 & -0.723166 \\
\hline 19 & 1 & 0 & -1.563567 & 1.729410 & 0.070590 \\
\hline 20 & 1 & 0 & -1.899868 & -1.138614 & -1.090692 \\
\hline
\end{tabular}

Energy: $\mathrm{HF}=-274.2838833$

Zero-Point Energy Correction: $\mathbf{0 . 1 8 2 5 7 5}$

Imaginary Frequency: Minimum (No imaginary frequencies) 
<smiles>CC1=CC=CC1C</smiles>

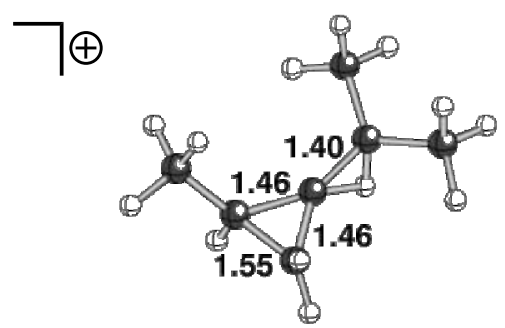

b3lyp/6-31G(d)

Coordinates:

Standard orientation:

\begin{tabular}{|c|c|c|c|c|c|}
\hline \multirow{2}{*}{$\begin{array}{l}\text { Center } \\
\text { Number }\end{array}$} & \multirow{2}{*}{$\begin{array}{l}\text { Atomic } \\
\text { Number }\end{array}$} & \multirow{2}{*}{\multicolumn{2}{|c|}{$\begin{array}{r}\text { Atomic } \\
\text { Type }\end{array}$}} & \multicolumn{2}{|c|}{ Coordinates (Angstrom } \\
\hline & & & & $\begin{array}{ll}X & Y\end{array}$ & $\mathrm{Z}$ \\
\hline 1 & 6 & 0 & -1.569184 & -0.052405 & -0.443488 \\
\hline 2 & 6 & 0 & -0.161401 & -0.368361 & -0.234069 \\
\hline 3 & 6 & 0 & -1.083996 & -1.483548 & -0.088081 \\
\hline 4 & 6 & 0 & 1.166854 & 0.074472 & -0.145859 \\
\hline 5 & 1 & 0 & 0.933203 & -0.292660 & -1.276765 \\
\hline 6 & 1 & 0 & -1.195572 & -1.892475 & 0.917638 \\
\hline 7 & 1 & 0 & -1.166211 & -2.224569 & -0.881592 \\
\hline 8 & 6 & 0 & 1.474727 & 1.563890 & -0.195988 \\
\hline 9 & 1 & 0 & 2.427373 & 1.742330 & -0.701156 \\
\hline 10 & 1 & 0 & 0.688793 & 2.134064 & -0.696542 \\
\hline 11 & 1 & 0 & 1.571221 & 1.930145 & 0.832215 \\
\hline 12 & 6 & 0 & 2.232033 & -0.845473 & 0.432540 \\
\hline 13 & 1 & 0 & 2.366514 & -0.591608 & 1.490035 \\
\hline 14 & 1 & 0 & 1.953181 & -1.899448 & 0.361244 \\
\hline 15 & 1 & 0 & 3.189996 & -0.689640 & -0.069946 \\
\hline 16 & 6 & 0 & -2.293525 & 0.861154 & 0.536570 \\
\hline 17 & 1 & 0 & -2.206591 & 1.907791 & 0.231143 \\
\hline 18 & 1 & 0 & -3.354262 & 0.592735 & 0.551297 \\
\hline 19 & 1 & 0 & -1.902060 & 0.759023 & 1.553145 \\
\hline 20 & 1 & 0 & -1.898632 & 0.025933 & -1.480462 \\
\hline
\end{tabular}

Energy: $\mathrm{HF}=-274.2316609$

Zero-Point Energy Correction: $\mathbf{0 . 1 7 8 3 9 3}$

Imaginary Frequency: One imaginary frequency $-401.3908 \mathrm{~cm}^{-1}$ 

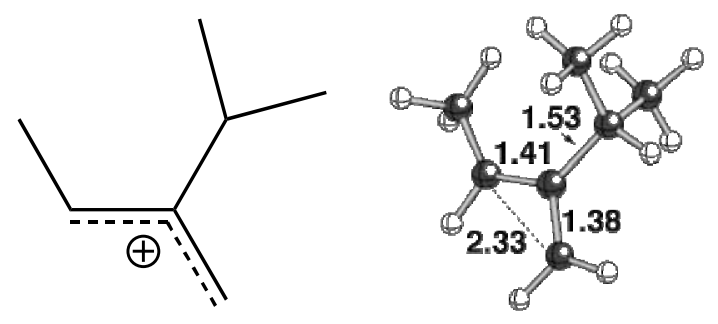

b3lyp/6-31G(d)

\section{Coordinates:}

Standard orientation:

\begin{tabular}{|c|c|c|c|c|c|}
\hline Center & Atomic & & tomic & Coordinate & is (Angstro \\
\hline Number & Numbe & & Type & X $\quad Y$ & $\mathrm{Z}$ \\
\hline 1 & 6 & 0 & -1.523704 & -0.130657 & -0.000016 \\
\hline 2 & 6 & 0 & -0.40 & 0.733745 & -0.000004 \\
\hline 3 & 6 & 0 & -0.760313 & 2.066857 & 0.000016 \\
\hline 4 & 6 & 0 & 74 & 0.308676 & 0.000007 \\
\hline 5 & 1 & 0 & 1.632984 & 1.244832 & 0.000031 \\
\hline 6 & 1 & 0 & -0.009 & 2.854728 & 0.000044 \\
\hline 7 & 1 & 0 & -1.7 & 2.391054 & 0.000010 \\
\hline 8 & 6 & 0 & & -0.449249 & 1.285145 \\
\hline 9 & 1 & 0 & 535871 & -0.609746 & 1.289245 \\
\hline 10 & 1 & 0 & & 0.120568 & 2.184446 \\
\hline 11 & 1 & 0 & 44 & -1.433618 & 1.358540 \\
\hline 12 & 6 & & & -0.449206 & -1.285147 \\
\hline 13 & 1 & & 0.980635 & -1.433610 & -1.358531 \\
\hline 14 & 1 & & 1.197909 & 0.120592 & -2.184443 \\
\hline 15 & 1 & 0 & 2.535899 & -0.609628 & -1.289279 \\
\hline 16 & 6 & & & -1.591661 & -0.000017 \\
\hline 17 & 1 & & & -1.915913 & 0.865681 \\
\hline 18 & 1 & 0 & -2.1799 & -1.915959 & -0.865525 \\
\hline 19 & 1 & 0 & -0.615011 & -2.099383 & -0.000117 \\
\hline 20 & 1 & 0 & -2.500916 & 0.355055 & -0.000014 \\
\hline
\end{tabular}

Energy: $\mathrm{HF}=-274.2661587$

Zero-Point Energy Correction: $\mathbf{0 . 1 8 2 1 0 2}$

Imaginary Frequency: Minimum (No imaginary frequencies) 


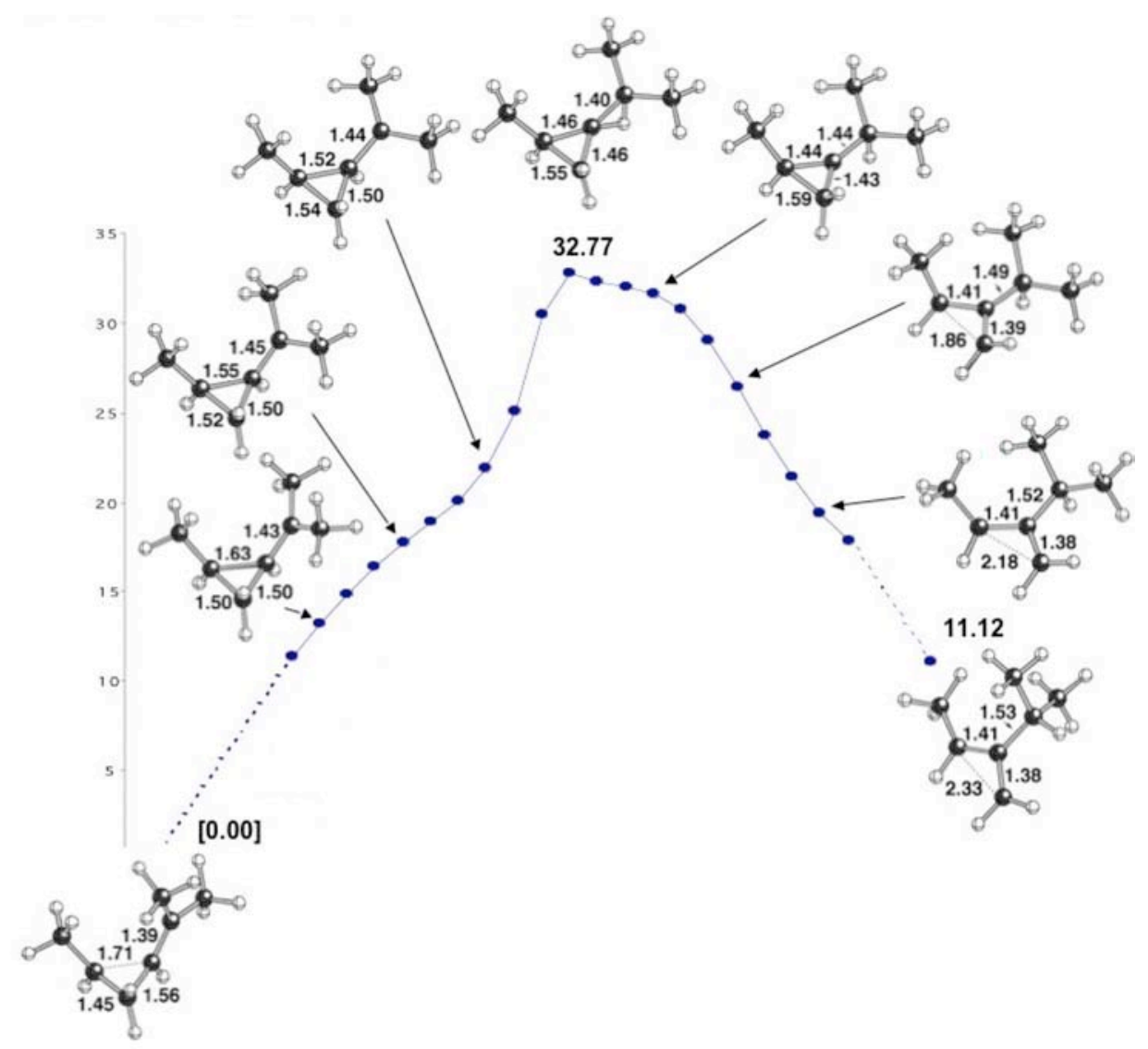

S136 


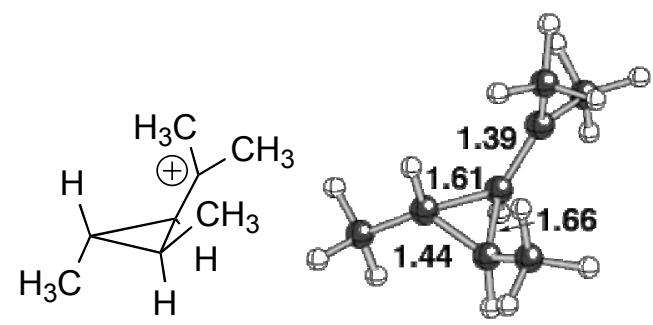

b3lyp/6-31G(d)

\section{Coordinates:}

Standard orientation:

\begin{tabular}{|c|c|c|c|c|c|}
\hline \multirow{2}{*}{$\begin{array}{l}\text { Center } \\
\text { Number }\end{array}$} & \multirow{2}{*}{\multicolumn{2}{|c|}{$\begin{array}{l}\text { Atomic } \\
\text { Number }\end{array}$}} & \multirow{2}{*}{$\begin{array}{l}\text { Atomic } \\
\text { Type }\end{array}$} & \multicolumn{2}{|c|}{ Coordinates (Angstrom } \\
\hline & & & & X $\quad Y$ & $\mathrm{Z}$ \\
\hline 1 & 6 & 0 & 1.289148 & -0.356602 & 0.453212 \\
\hline 2 & 6 & 0 & -0.033766 & -0.499454 & -0.454529 \\
\hline 3 & 6 & 0 & 1.172361 & 0.635855 & -0.584782 \\
\hline 4 & 1 & 0 & 1.662431 & 0.393746 & -1.527711 \\
\hline 5 & 6 & 0 & -1.316040 & -0.230854 & 0.007289 \\
\hline 6 & 6 & 0 & 0.923678 & 2.106404 & -0.379078 \\
\hline 7 & 1 & 0 & 1.896811 & 2.608540 & -0.451441 \\
\hline 8 & 1 & 0 & 0.506301 & 2.348796 & 0.599619 \\
\hline 9 & 1 & 0 & 0.056365 & -1.244382 & -1.238153 \\
\hline 10 & 1 & 0 & 0.285453 & 2.524845 & -1.162955 \\
\hline 11 & 6 & 0 & 2.226862 & -1.540091 & 0.372065 \\
\hline 12 & 1 & 0 & 2.412505 & -1.844975 & -0.662497 \\
\hline 13 & 1 & 0 & 3.188087 & -1.255537 & 0.816195 \\
\hline 14 & 1 & 0 & 1.835620 & -2.395278 & 0.929927 \\
\hline 15 & 1 & 0 & 1.047019 & -0.024068 & 1.460944 \\
\hline 16 & 6 & 0 & -1.661241 & 0.719175 & 1.102639 \\
\hline 17 & 1 & 0 & -0.841986 & 0.940179 & 1.786786 \\
\hline 18 & 1 & 0 & -1.987999 & 1.667254 & 0.646895 \\
\hline 19 & 1 & 0 & -2.518727 & 0.346666 & 1.672822 \\
\hline 20 & 6 & 0 & -2.466538 & -0.944716 & -0.620255 \\
\hline 21 & 1 & 0 & -3.302733 & -0.255713 & -0.792063 \\
\hline 22 & 1 & 0 & -2.205336 & -1.452322 & -1.550489 \\
\hline 23 & 1 & 0 & -2.840588 & -1.696047 & 0.092758 \\
\hline
\end{tabular}

Energy: $\mathrm{HF}=-313.6056341$

Zero-Point Energy Correction: 0.210563

Imaginary Frequency: Minimum (No imaginary frequencies) 


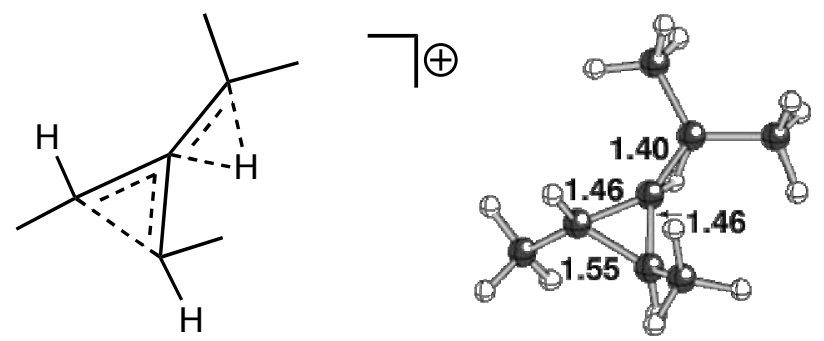

b3lyp/6-31G(d)

Coordinates:

Standard orientation:

\begin{tabular}{|c|c|c|c|c|}
\hline \multirow{2}{*}{$\begin{array}{l}\text { Center } \\
\text { Number }\end{array}$} & \multirow{2}{*}{$\begin{array}{l}\text { Atomic } \\
\text { Number }\end{array}$} & \multirow{2}{*}{$\begin{array}{r}\text { Atomic } \\
\text { Type }\end{array}$} & \multicolumn{2}{|c|}{ Coordinates (Angstror } \\
\hline & & & X $\quad Y$ & I $\quad \mathrm{Z}$ \\
\hline 1 & 6 & 1.279137 & -0.644358 & 0.352254 \\
\hline 2 & 6 & 0.015933 & -0.028177 & -0.044429 \\
\hline 3 & 6 & 1.219841 & 0.761790 & -0.296341 \\
\hline 4 & 1 & 1.577753 & 0.760310 & -1.328121 \\
\hline 5 & 6 & -1.380446 & -0.128890 & -0.114776 \\
\hline 6 & 6 & 1.458062 & 2.059826 & 0.464739 \\
\hline 7 & 1 & 2.534828 & 2.245582 & 0.523440 \\
\hline 8 & 1 & 1.065260 & 2.009268 & 1.484759 \\
\hline 9 & 1 & -0.858967 & -0.579826 & -1.120261 \\
\hline 10 & 1 & 0.990882 & 2.906494 & -0.046779 \\
\hline 11 & 6 & 1.915673 & -1.810370 & -0.397161 \\
\hline 12 & 1 & 1.755775 & -1.727876 & -1.477286 \\
\hline 13 & 1 & 2.992927 & -1.813936 & -0.210352 \\
\hline 14 & 1 & 1.503873 & -2.764213 & -0.054406 \\
\hline 15 & 1 & 1.400652 & -0.687165 & 1.438989 \\
\hline 16 & 6 & -2.096515 & -1.267115 & 0.596030 \\
\hline 17 & 1 & -1.439593 & -2.120040 & 0.781117 \\
\hline 18 & 1 & -2.459481 & -0.892674 & 1.559865 \\
\hline 19 & 1 & -2.965522 & -1.596753 & 0.020827 \\
\hline 20 & 6 & -2.212027 & 1.077827 & -0.520961 \\
\hline 21 & 1 & -2.580414 & 1.562936 & 0.390022 \\
\hline 22 & 1 & -1.633422 & 1.806679 & -1.092963 \\
\hline 23 & 1 & -3.082492 & 0.768011 & -1.104984 \\
\hline
\end{tabular}

Energy: $\mathrm{HF}=-313.5516739$

Zero-Point Energy Correction: $\mathbf{0 . 2 0 6 7 8 6}$

Imaginary Frequency: One imaginary frequency $-414.5650 \mathrm{~cm}^{-1}$ 
b3lyp/6-31G(d)
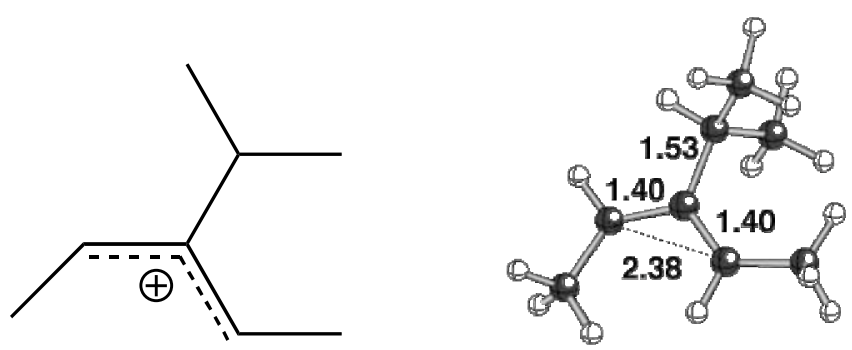

\section{Coordinates:}

Standard orientation:

\begin{tabular}{|c|c|c|c|c|c|}
\hline \multirow{2}{*}{$\begin{array}{l}\text { Center } \\
\text { Number }\end{array}$} & \multirow{2}{*}{\multicolumn{2}{|c|}{$\begin{array}{l}\text { Atomic } \\
\text { Number }\end{array}$}} & \multirow{2}{*}{$\begin{array}{l}\text { Atomic } \\
\text { Type }\end{array}$} & \multicolumn{2}{|c|}{ Coordinates (Angstron } \\
\hline & & & & X $\quad Y$ & $\mathrm{Z}$ \\
\hline 1 & 6 & 0 & -1.579269 & -0.833932 & -0.000221 \\
\hline 2 & 6 & 0 & -0.371346 & -0.123576 & -0.000278 \\
\hline 3 & 6 & 0 & -0.467756 & 1.269735 & 0.000008 \\
\hline 4 & 1 & 0 & -1.470248 & 1.692475 & -0.000384 \\
\hline 5 & 6 & 0 & 0.941256 & -0.917339 & -0.000405 \\
\hline 6 & 6 & 0 & 0.597413 & 2.282189 & 0.000752 \\
\hline 7 & 1 & 0 & 0.449113 & 2.944809 & -0.868202 \\
\hline 8 & 1 & 0 & 1.612822 & 1.896423 & 0.002118 \\
\hline 9 & 1 & 0 & 0.643289 & -1.973432 & -0.001103 \\
\hline 10 & 1 & 0 & 0.447102 & 2.945917 & 0.868466 \\
\hline 11 & 6 & 0 & -2.983593 & -0.384036 & -0.000175 \\
\hline 12 & 1 & 0 & -3.155463 & 0.691867 & -0.000904 \\
\hline 13 & 1 & 0 & -3.489894 & -0.832573 & -0.869558 \\
\hline 14 & 1 & ] & -3.489340 & -0.831206 & 0.870247 \\
\hline 15 & 1 & 0 & -1.476893 & -1.920967 & -0.000129 \\
\hline 16 & 6 & 0 & 1.766435 & -0.700404 & -1.284863 \\
\hline 17 & 1 & 0 & 1.172997 & -0.900892 & -2.183162 \\
\hline 18 & 1 & 0 & 2.169794 & 0.313673 & -1.365323 \\
\hline 19 & 1 & 0 & 2.617446 & -1.388279 & -1.287708 \\
\hline 20 & 6 & 0 & 1.765577 & -0.702006 & 1.284954 \\
\hline 21 & 1 & 0 & 2.169004 & 0.311917 & 1.366906 \\
\hline 22 & 1 & 0 & 1.171460 & -0.903536 & 2.182564 \\
\hline 23 & 1 & 0 & 2.616509 & -1.389981 & 1.287543 \\
\hline
\end{tabular}

Energy: $\mathrm{HF}=-313.5965141$

Zero-Point Energy Correction: 0.210328

Imaginary Frequency: Minimum (No imaginary frequencies) 


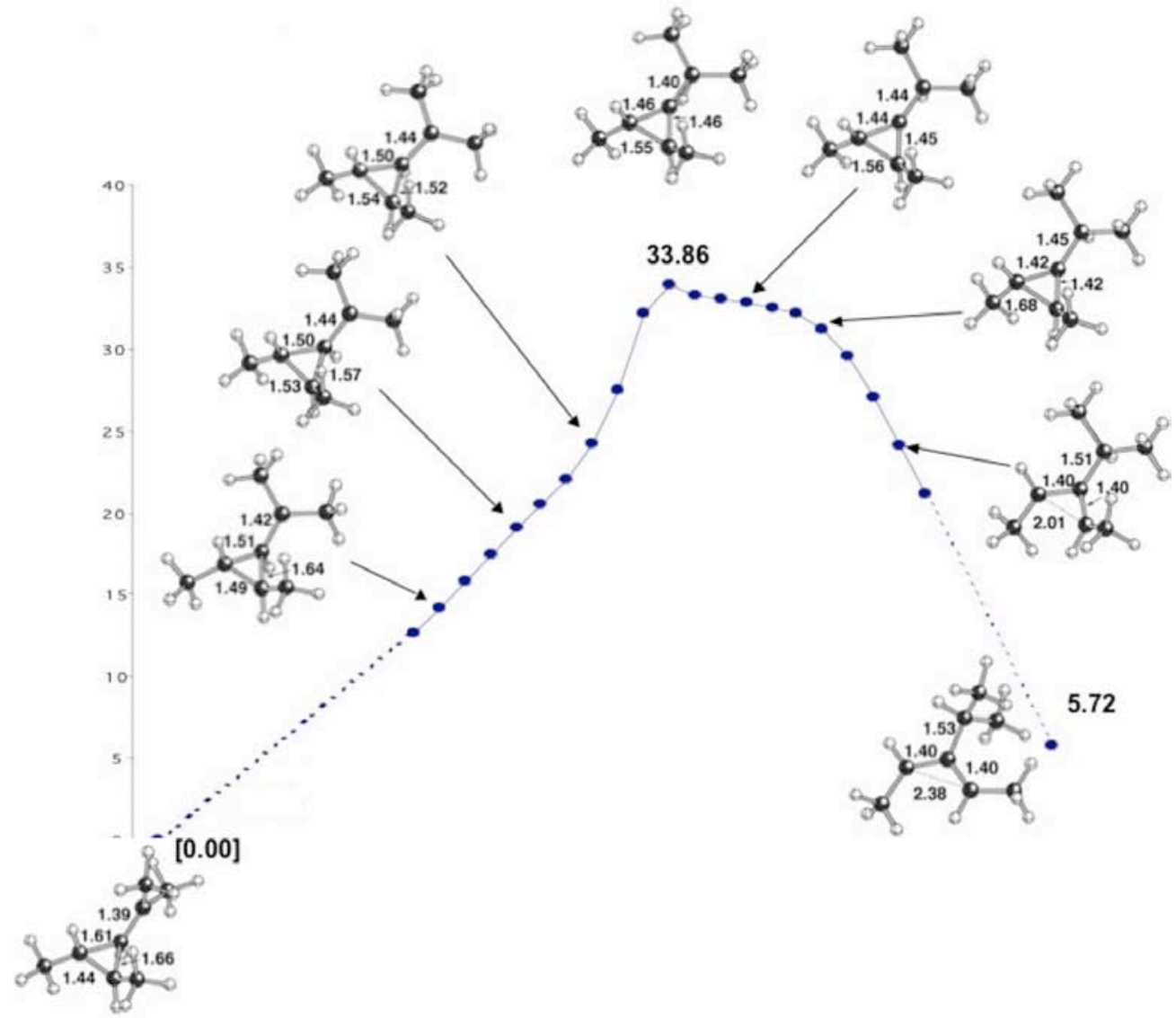




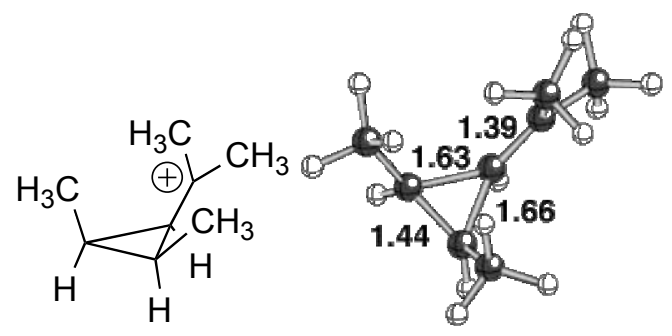

b3lyp/6-31G(d)

\section{Coordinates:}

Standard orientation:

\begin{tabular}{cccccc} 
Center & \multicolumn{2}{c}{ Atomic } & \multicolumn{2}{c}{ Atomic } & \multicolumn{3}{c}{ Coordinates } & Angstroms \\
Number & Number & Type & X & Y & $Z$ \\
\hline 1 & 6 & 0 & 1.237894 & -0.776633 & -0.623543 \\
2 & 6 & 0 & -0.246007 & -0.163978 & -0.902061 \\
3 & 6 & 0 & 1.190076 & 0.657313 & -0.788537 \\
4 & 1 & 0 & 1.358409 & 1.027252 & -1.798865 \\
5 & 6 & 0 & -1.298562 & -0.042651 & -0.002370 \\
6 & 6 & 0 & 1.459074 & 1.683751 & 0.279273 \\
7 & 1 & 0 & 2.509752 & 1.984717 & 0.180358 \\
8 & 6 & 0 & -2.690773 & -0.108403 & -0.537817 \\
9 & 1 & 0 & -3.160301 & -1.035180 & -0.173307 \\
10 & 1 & 0 & 1.320635 & 1.319255 & 1.297659 \\
11 & 1 & 0 & -0.534015 & -0.290118 & -1.939699 \\
12 & 1 & 0 & -3.295881 & 0.709715 & -0.125719 \\
13 & 1 & 0 & 0.851765 & 2.581658 & 0.131028 \\
14 & 1 & 0 & -2.743247 & -0.094788 & -1.627630 \\
15 & 6 & 0 & 1.622830 & -1.510797 & 0.637928 \\
16 & 1 & 0 & 1.025385 & -2.416648 & 0.774172 \\
17 & 1 & 0 & 2.667583 & -1.823148 & 0.521569 \\
18 & 1 & 0 & 1.573570 & -0.905398 & 1.544317 \\
19 & 1 & 0 & 1.460856 & -1.341545 & -1.527870 \\
20 & 6 & 0 & -1.191640 & 0.175034 & 1.468898 \\
21 & 1 & 0 & -0.324650 & -0.299585 & 1.926391 \\
22 & 1 & 0 & -1.106888 & 1.258467 & 1.648509 \\
23 & 1 & 0 & -2.100322 & -0.156474 & 1.978455 \\
-------------------------------------------------------------
\end{tabular}

Energy: $\mathrm{HF}=-313.5987413$ 

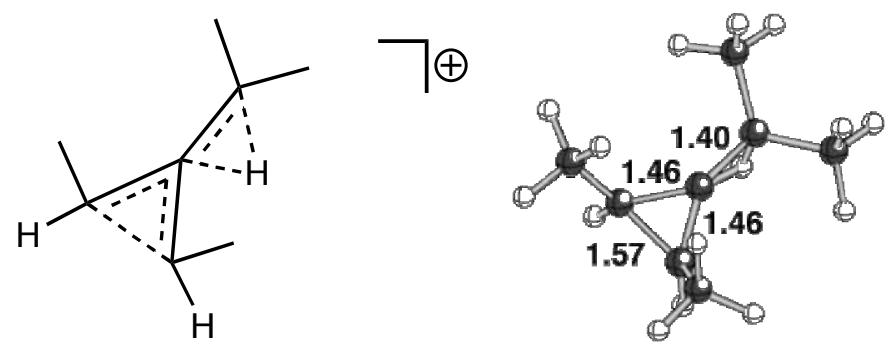

b3lyp/6-31G(d)

\section{Coordinates:}

Standard orientation:

\begin{tabular}{|c|c|c|c|c|}
\hline \multirow{2}{*}{$\begin{array}{l}\text { Center } \\
\text { Number }\end{array}$} & Atomic & Atomic & \multicolumn{2}{|c|}{ Coordinates (Angstron } \\
\hline & Numbe & r Type & X $\quad Y$ & $\mathrm{Z}$ \\
\hline 1 & 6 & -1.200567 & -0.787153 & 0.603848 \\
\hline 2 & 6 & 0.018494 & -0.000013 & 0.467576 \\
\hline 3 & 6 & -1.200518 & 0.787200 & 0.603842 \\
\hline 4 & 1 & -1.426193 & 1.165538 & 1.600704 \\
\hline 5 & 6 & 1.382614 & -0.000023 & 0.150054 \\
\hline 6 & 6 & -1.678051 & 1.670549 & -0.538306 \\
\hline 7 & 1 & -2.768156 & 1.756985 & -0.494155 \\
\hline 8 & 6 & 13 & 1.299164 & -0.193464 \\
\hline 9 & 1 & 7 & 1.30 & 3706 \\
\hline 10 & 1 & -1.40 & 1.265 & 17567 \\
\hline 11 & 1 & 150930 & -0.000010 & 1.366282 \\
\hline 12 & 1 & 2.1602 & 1.369952 & -1.285040 \\
\hline 13 & 1 & -1.2 & 2.676184 & -0.452312 \\
\hline 14 & 1 & 0 & 2.17 & 0.176186 \\
\hline 15 & 6 & -1.678152 & -1.670510 & -0.538274 \\
\hline 16 & 1 & -1.255644 & -2.676213 & -0.452155 \\
\hline 17 & 1 & -2.768277 & -1.756748 & -0.494218 \\
\hline 18 & 1 & & -1.265582 & -1.517544 \\
\hline 19 & 1 & -1.42 & -1.165 & 1.600723 \\
\hline 20 & 6 & 2.088784 & -1.299219 & -0.193496 \\
\hline 21 & 1 & 1.551006 & -2.175070 & 0.176130 \\
\hline 22 & 1 & 2.160235 & -1.369977 & -1.285075 \\
\hline 23 & 1 & 3.107239 & -1.303904 & 0.203668 \\
\hline
\end{tabular}

Energy: $\mathrm{HF}=-313.551277$

Zero-Point Energy Correction: 0.206719

Imaginary Frequency: One imaginary frequency $-496.3121 \mathrm{~cm}^{-1}$ 

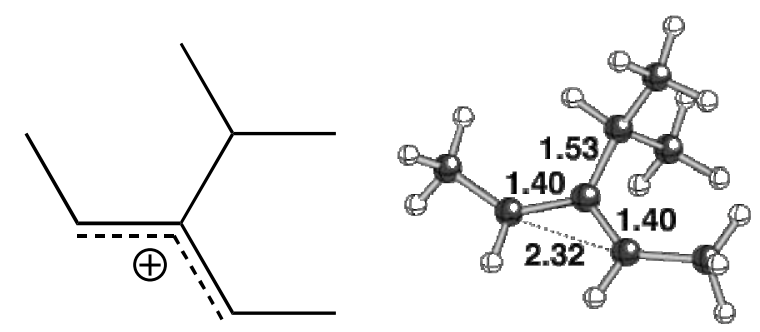

b3lyp/6-31G(d)

\section{Coordinates:}

Standard orientation:

\begin{tabular}{|c|c|c|c|c|c|}
\hline \multirow{2}{*}{$\begin{array}{l}\text { Center } \\
\text { Number }\end{array}$} & \multirow{2}{*}{$\begin{array}{l}\text { Atomic } \\
\text { Number }\end{array}$} & \multirow{2}{*}{\multicolumn{2}{|c|}{$\begin{array}{l}\text { Atomic } \\
\text { Type }\end{array}$}} & \multicolumn{2}{|c|}{ Coordinates (Angstroms) } \\
\hline & & & & X $\quad Y$ & $\mathrm{Z}$ \\
\hline 1 & 6 & 0 & 1.635968 & 0.828896 & -0.002979 \\
\hline 2 & 6 & 0 & 0.296822 & 0.419504 & -0.002647 \\
\hline 3 & 6 & 0 & -0.583998 & 1.508078 & -0.000183 \\
\hline 4 & 1 & 0 & -0.125290 & 2.497819 & -0.002697 \\
\hline 5 & 6 & 0 & -0.124215 & -1.049253 & -0.002932 \\
\hline 6 & 6 & 0 & -2.053373 & 1.537190 & 0.005918 \\
\hline 7 & 1 & 0 & -2.393513 & 2.122021 & -0.864746 \\
\hline 8 & 6 & 0 & -0.888290 & -1.440703 & -1.285503 \\
\hline 9 & 1 & 0 & -1.054235 & -2.522245 & -1.288978 \\
\hline 10 & 1 & 0 & -2.386734 & 2.131113 & 0.872871 \\
\hline 11 & 1 & 0 & 0.799219 & -1.635300 & -0.009401 \\
\hline 12 & 1 & 0 & -1.869205 & -0.961721 & -1.359250 \\
\hline 13 & 1 & 0 & -2.543004 & 0.567330 & 0.012667 \\
\hline 14 & 1 & 0 & -0.319125 & -1.187641 & -2.186133 \\
\hline 15 & 6 & 0 & 2.859272 & 0.014104 & -0.000324 \\
\hline 16 & 1 & 0 & 2.706271 & -1.063858 & 0.004553 \\
\hline 17 & 1 & 0 & 3.472128 & 0.297549 & -0.871736 \\
\hline 18 & 1 & 0 & 3.474108 & 0.305446 & 0.866953 \\
\hline 19 & 1 & 0 & 1.818419 & 1.904266 & -0.004897 \\
\hline 20 & 6 & 0 & -0.873912 & -1.445439 & 1.286633 \\
\hline 21 & 1 & 0 & -0.294121 & -1.196972 & 2.181749 \\
\hline 22 & 1 & 0 & -1.853196 & -0.965321 & 1.373809 \\
\hline 23 & 1 & 0 & -1.041367 & -2.526759 & 1.287346 \\
\hline
\end{tabular}

Energy: $\mathrm{HF}=-313.600431$ 


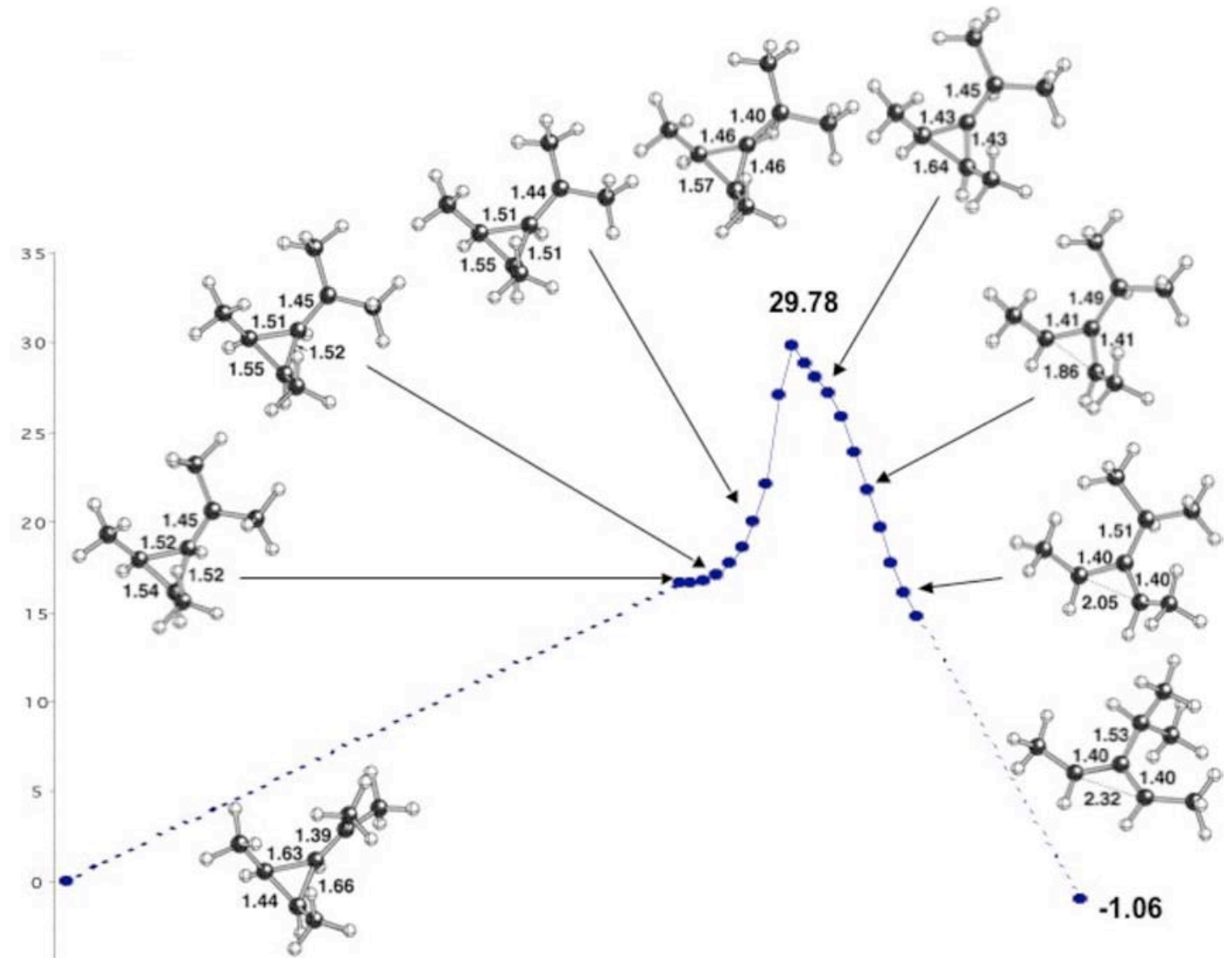




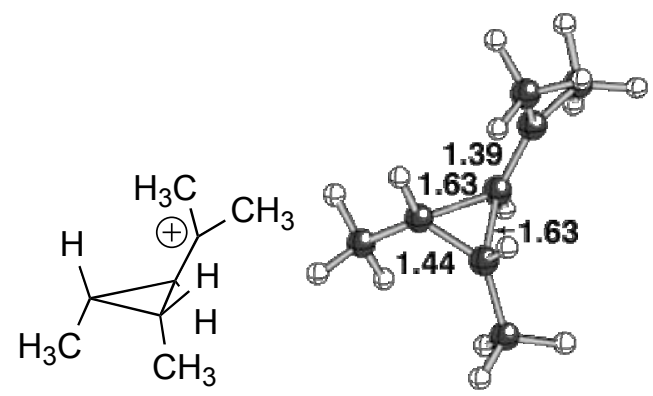

b3lyp/6-31G(d)

\section{Coordinates:}

Standard orientation:

\begin{tabular}{|c|c|c|c|c|c|}
\hline \multirow{2}{*}{$\begin{array}{l}\text { Center } \\
\text { Number }\end{array}$} & \multirow{2}{*}{\multicolumn{2}{|c|}{$\begin{array}{l}\text { Atomic } \\
\text { Number }\end{array}$}} & \multirow{2}{*}{$\begin{array}{r}\text { Atomic } \\
\text { Type }\end{array}$} & \multicolumn{2}{|c|}{ Coordinates (Angstrom } \\
\hline & & & & X $\quad Y$ & $\mathrm{Z}$ \\
\hline 1 & 6 & 0 & 1.015918 & -0.745316 & 0.452076 \\
\hline 2 & 6 & 0 & -0.114759 & 0.024683 & -0.436888 \\
\hline 3 & 6 & 0 & 1.016867 & 0.695922 & 0.528748 \\
\hline 4 & 6 & 0 & -1.456807 & 0.002358 & -0.085849 \\
\hline 5 & 6 & 0 & -2.494357 & 0.089577 & -1.154286 \\
\hline 6 & 1 & 0 & -3.273909 & -0.667032 & -1.001407 \\
\hline 7 & 1 & 0 & 0.137923 & 0.084361 & -1.490323 \\
\hline 8 & 1 & 0 & -3.002398 & 1.062742 & -1.074079 \\
\hline 9 & 1 & 0 & -2.082259 & -0.003752 & -2.160635 \\
\hline 10 & 6 & 0 & 1.989818 & -1.579115 & -0.345179 \\
\hline 11 & 1 & 0 & 2.847728 & -1.811633 & 0.297940 \\
\hline 12 & 1 & 0 & 1.534039 & -2.525026 & -0.650125 \\
\hline 13 & 1 & 0 & 2.366009 & -1.067387 & -1.233755 \\
\hline 14 & 6 & 0 & 1.991215 & 1.608553 & -0.175880 \\
\hline 15 & 1 & 0 & 1.531925 & 2.577047 & -0.391947 \\
\hline 16 & 1 & 0 & 2.841751 & 1.782859 & 0.494843 \\
\hline 17 & 1 & 0 & 2.379343 & 1.187664 & -1.106023 \\
\hline 18 & 1 & 0 & 0.556526 & -1.266115 & 1.289179 \\
\hline 19 & 1 & 0 & 0.558015 & 1.126430 & 1.415790 \\
\hline 20 & 6 & 0 & -1.952966 & -0.104079 & 1.316001 \\
\hline 21 & 1 & 0 & -1.179814 & -0.035310 & 2.082190 \\
\hline 22 & 1 & 0 & -2.711842 & 0.665941 & 1.503422 \\
\hline 23 & 1 & 0 & -2.472609 & -1.066292 & 1.432476 \\
\hline
\end{tabular}

Energy: $\mathrm{HF}=-313.6089557$

Zero-Point Energy Correction: 0.210462

Imaginary Frequency: Minimum (No imaginary frequencies) 
b3lyp/6-31G(d)
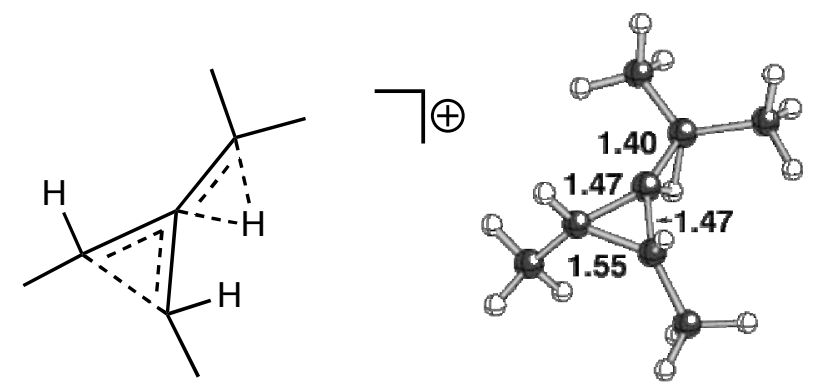

\section{Coordinates:}

Standard orientation:

\begin{tabular}{|c|c|c|c|c|}
\hline \multirow{2}{*}{$\begin{array}{l}\text { Center } \\
\text { Number }\end{array}$} & \multirow{2}{*}{$\begin{array}{l}\text { Atomic } \\
\text { Number }\end{array}$} & \multirow{2}{*}{$\begin{array}{l}\text { Atomic } \\
\text { Type }\end{array}$} & \multicolumn{2}{|c|}{ Coordinates (Angstron } \\
\hline & & & X $\quad Y$ & $\mathrm{Z}$ \\
\hline 1 & 6 & 1.148177 & -0.774056 & 0.617226 \\
\hline 2 & 6 & -0.070382 & -0.000023 & 0.358087 \\
\hline 3 & 6 & 1.148135 & 0.774066 & 0.617240 \\
\hline 4 & 6 & -1.405600 & -0.000013 & -0.060357 \\
\hline 5 & 6 & -2.191503 & 1.299408 & -0.103808 \\
\hline 6 & 1 & -2.875070 & 1.312384 & -0.956797 \\
\hline 7 & 1 & -0.613320 & -0.000025 & -1.001854 \\
\hline 8 & 1 & -2.798268 & 1.358106 & 0.807049 \\
\hline 9 & 1 & -1.542378 & 2.176872 & -0.143445 \\
\hline 10 & 6 & 1.834221 & -1.624407 & -0.445701 \\
\hline 11 & 1 & 2.889042 & -1.746449 & -0.184073 \\
\hline 12 & 1 & 1.378698 & -2.617925 & -0.493347 \\
\hline 13 & 1 & 1.780844 & -1.173040 & -1.440625 \\
\hline 14 & 6 & 1.834149 & 1.624442 & -0.445685 \\
\hline 15 & 1 & 1.378624 & 2.617961 & -0.493293 \\
\hline 16 & 1 & 2.888978 & 1.746480 & -0.184086 \\
\hline 17 & 1 & 1.780743 & 1.173102 & -1.440621 \\
\hline 18 & 1 & 1.156936 & -1.225605 & 1.612475 \\
\hline 19 & 1 & 1.156870 & 1.225615 & 1.612489 \\
\hline 20 & 6 & -2.191516 & -1.299428 & -0.103794 \\
\hline 21 & 1 & -1.542403 & -2.176904 & -0.143363 \\
\hline 22 & 1 & -2.798317 & -1.358072 & 0.807043 \\
\hline 23 & 1 & -2.875053 & -1.312430 & -0.956808 \\
\hline
\end{tabular}

Energy: $H F=-313.5496013$

Zero-Point Energy Correction: 0.206830

Imaginary Frequency: One imaginary frequency $-406.7422 \mathrm{~cm}^{-1}$ 
b3lyp/6-31G(d)

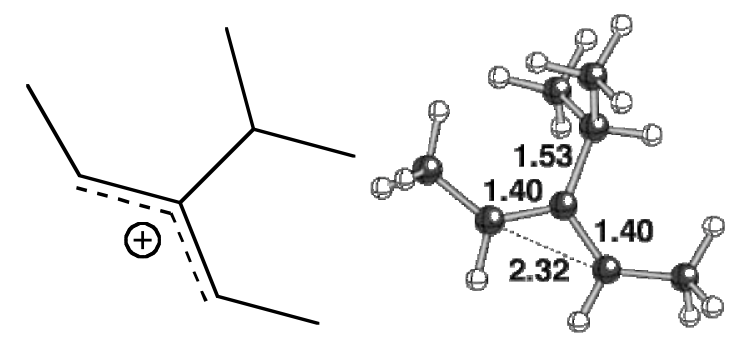

\section{Coordinates:}

Standard orientation:

\begin{tabular}{|c|c|c|c|c|}
\hline \multirow{2}{*}{$\begin{array}{l}\text { Center } \\
\text { Number }\end{array}$} & \multirow{2}{*}{$\begin{array}{l}\text { Atomic } \\
\text { Number }\end{array}$} & \multirow{2}{*}{$\begin{array}{r}\text { Atomic } \\
\text { Type }\end{array}$} & \multicolumn{2}{|c|}{ Coordinates (Angstroms } \\
\hline & & & X $\quad Y$ & $\mathrm{Z}$ \\
\hline 1 & 6 & 0.584052 & 1.508075 & 0.000193 \\
\hline 2 & 6 & -0.296796 & 0.419531 & -0.003980 \\
\hline 3 & 6 & -1.635930 & 0.828963 & -0.004489 \\
\hline 4 & 6 & 0.124178 & -1.049251 & -0.004847 \\
\hline 5 & 6 & 0.869092 & -1.447340 & 1.286894 \\
\hline 6 & 1 & 1.037255 & -2.528552 & 1.286324 \\
\hline 7 & 1 & -0.799261 & -1.635226 & -0.015737 \\
\hline 8 & 1 & 1.847707 & -0.966706 & 1.378740 \\
\hline 9 & 1 & 0.285703 & -1.200816 & 2.180205 \\
\hline 10 & 6 & 2.053415 & 1.537150 & 0.009549 \\
\hline 11 & 1 & 2.395223 & 2.117803 & -0.863346 \\
\hline 12 & 1 & 2.385197 & 2.135142 & 0.874211 \\
\hline 13 & 1 & 2.542970 & 0.567298 & 0.021487 \\
\hline 14 & 6 & -2.859238 & 0.014179 & -0.000927 \\
\hline 15 & 1 & -3.475843 & 0.308605 & 0.863973 \\
\hline 16 & 1 & -3.470313 & 0.294484 & -0.874673 \\
\hline 17 & 1 & -2.706230 & -1.063759 & 0.007844 \\
\hline 18 & 1 & 0.125379 & 2.497828 & -0.003585 \\
\hline 19 & 1 & -1.818361 & 1.904334 & -0.007256 \\
\hline 20 & 6 & 0.892968 & -1.438891 & -1.285150 \\
\hline 21 & 1 & 0.327339 & -1.184109 & -2.187521 \\
\hline 22 & 1 & 1.874406 & -0.960323 & -1.354355 \\
\hline 23 & 1 & 1.058386 & -2.520509 & -1.289770 \\
\hline
\end{tabular}

Energy: $\mathrm{HF}=-313.600431$

Zero-Point Energy Correction: 0.210341

Imaginary Frequency: Minimum (No imaginary frequencies) 


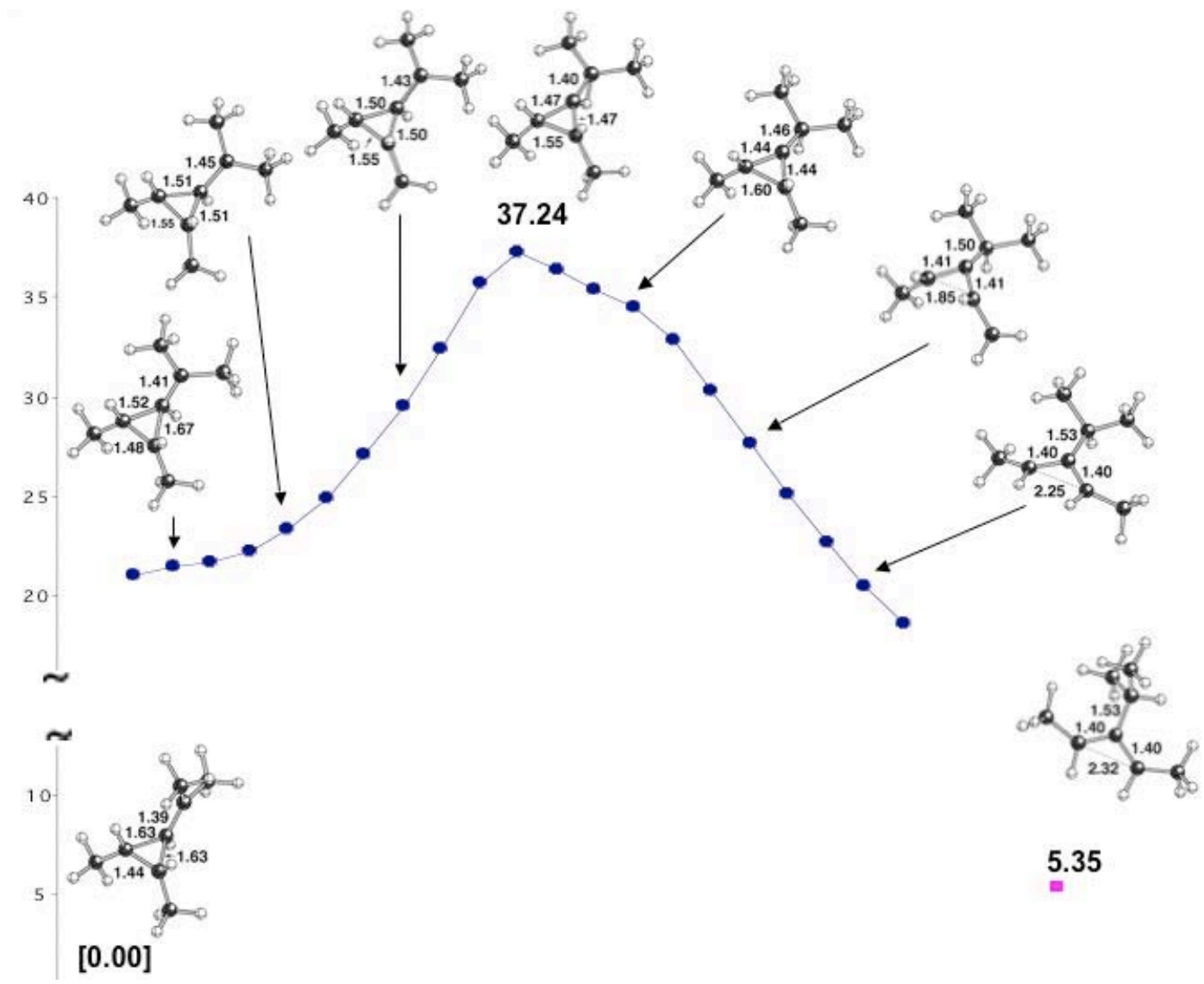



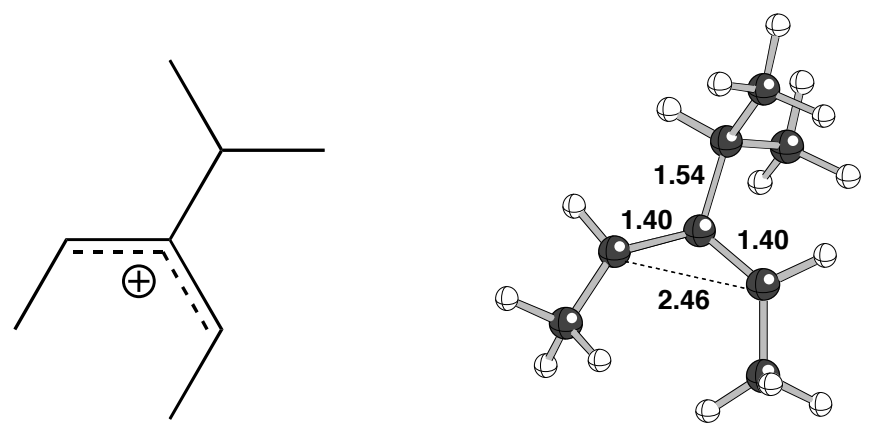

b31yp/6-31G(d)

Coordinates:

Standard orientation:

\begin{tabular}{|c|c|c|c|c|c|}
\hline \multirow{2}{*}{$\begin{array}{l}\text { Center } \\
\text { Number }\end{array}$} & \multirow{2}{*}{\multicolumn{2}{|c|}{$\begin{array}{l}\text { Atomic } \\
\text { Number }\end{array}$}} & \multirow{2}{*}{$\begin{array}{l}\text { Atomic } \\
\text { Type }\end{array}$} & \multicolumn{2}{|c|}{ Coordinates (Angstrom } \\
\hline & & & & $\begin{array}{ll}X & Y\end{array}$ & Z \\
\hline 1 & 6 & 0 & 0.991243 & -1.218724 & -0.197137 \\
\hline 2 & 6 & 0 & 0.079711 & -0.174264 & -0.033123 \\
\hline 3 & 6 & 0 & 0.455831 & 1.156863 & 0.167758 \\
\hline 4 & 6 & 0 & -1.426448 & -0.499806 & -0.070459 \\
\hline 5 & 6 & 0 & -2.095232 & -0.204167 & 1.285071 \\
\hline 6 & 1 & 0 & -3.144545 & -0.512779 & 1.249343 \\
\hline 7 & 1 & 0 & -1.612173 & -0.744712 & 2.105581 \\
\hline 8 & 1 & 0 & -2.085577 & 0.865768 & 1.526909 \\
\hline 9 & 1 & 0 & -1.510058 & -1.578911 & -0.247089 \\
\hline 10 & 1 & 0 & 0.540631 & -2.172241 & -0.478800 \\
\hline 11 & 1 & 0 & -0.367477 & 1.828132 & 0.415747 \\
\hline 12 & 6 & 0 & 1.751194 & 1.846945 & 0.030794 \\
\hline 13 & 1 & 0 & 2.540739 & 1.284593 & -0.462277 \\
\hline 14 & 1 & 0 & 1.588822 & 2.795090 & -0.500898 \\
\hline 15 & 1 & 0 & 2.091954 & 2.155166 & 1.035804 \\
\hline 16 & 6 & 0 & 2.449560 & -1.297863 & 0.016182 \\
\hline 17 & 1 & 0 & 2.680000 & -2.241076 & 0.529907 \\
\hline 18 & 1 & 0 & 2.944223 & -1.392948 & -0.967164 \\
\hline 19 & 1 & 0 & 2.894456 & -0.469834 & 0.563674 \\
\hline 20 & 6 & 0 & -2.135604 & 0.220197 & -1.231588 \\
\hline 21 & 1 & 0 & -1.676571 & -0.014608 & -2.197756 \\
\hline 22 & 1 & 0 & -3.184865 & -0.086868 & -1.274754 \\
\hline 23 & 1 & 0 & -2.121085 & 1.310144 & -1.103211 \\
\hline
\end{tabular}

Energy: HF $=-313.5908138$

Zero-Point Energy Correction: 0.209934

Imaginary Frequency: Minimum (No imaginary frequencies) 

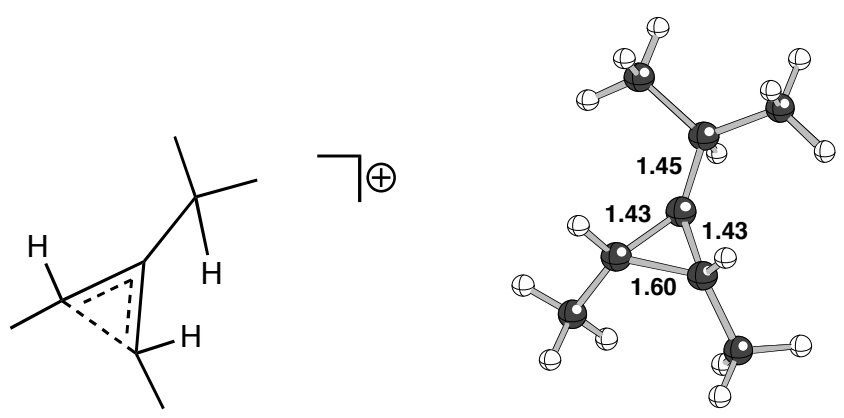

b3lyp/6-31G(d)

\section{Coordinates:}

Standard orientation:

\begin{tabular}{|c|c|c|c|c|}
\hline \multirow{2}{*}{$\begin{array}{l}\text { Center } \\
\text { Number }\end{array}$} & Atomic & \multirow{2}{*}{$\begin{array}{r}\text { Atomic } \\
\text { Type }\end{array}$} & \multicolumn{2}{|c|}{ Coordinates (Angstron } \\
\hline & Number & & $\begin{array}{ll}X & Y\end{array}$ & $\mathrm{Z}$ \\
\hline 1 & 6 & 0.984582 & 0.801469 & 0.510162 \\
\hline 2 & 6 & -0.080164 & -0.000002 & -0.015536 \\
\hline 3 & 6 & 0.984586 & -0.801469 & 0.510161 \\
\hline 4 & 6 & -1.487865 & -0.000001 & -0.343395 \\
\hline 5 & 6 & -2.237247 & -1.301441 & 0.002271 \\
\hline 6 & 1 & -3.205205 & -1.312380 & -0.503992 \\
\hline 7 & 1 & -1.677609 & -2.189643 & -0.304045 \\
\hline 8 & 1 & -2.420250 & -1.353732 & 1.080437 \\
\hline 9 & 1 & -1.332538 & 0.000002 & -1.459510 \\
\hline 10 & 1 & 0.662703 & 1.362773 & 1.395417 \\
\hline 11 & 1 & 0.662705 & -1.362775 & 1.395414 \\
\hline 12 & 6 & 2.081069 & -1.538160 & -0.266254 \\
\hline 13 & 1 & 2.993660 & -1.569133 & 0.333758 \\
\hline 14 & 1 & 1.744967 & -2.563914 & -0.443066 \\
\hline 15 & 1 & 2.301692 & -1.077284 & -1.229038 \\
\hline 16 & 6 & 2.081058 & 1.538165 & -0.266259 \\
\hline 17 & 1 & 1.744949 & 2.563917 & -0.443071 \\
\hline 18 & 1 & 2.993653 & 1.569144 & 0.333747 \\
\hline 19 & 1 & 2.301677 & 1.077289 & -1.229044 \\
\hline 20 & 6 & -2.237244 & 1.301438 & 0.002278 \\
\hline 21 & 1 & -1.677604 & 2.189641 & -0.304034 \\
\hline 22 & 1 & -3.205202 & 1.312382 & -0.503984 \\
\hline 23 & 1 & -2.420246 & 1.353725 & 1.080445 \\
\hline
\end{tabular}

Energy: $\mathrm{HF}=-313.5494628$

Zero-Point Energy Correction: 0.208497

Imaginary Frequency: One imaginary frequency $-111.6633 \mathrm{~cm}^{-1}$ 

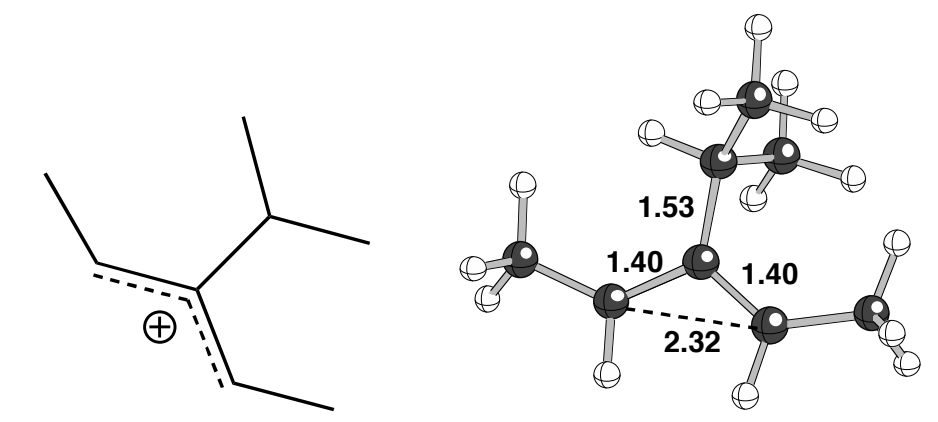

b3lyp/6-31G(d)

\section{Coordinates:}

Standard orientation:

\begin{tabular}{|c|c|c|c|c|}
\hline \multirow{2}{*}{$\begin{array}{l}\text { Center } \\
\text { Number }\end{array}$} & Atomic & Atomic & \multicolumn{2}{|c|}{ Coordinates (Angstror } \\
\hline & Number & r Type & X $\quad Y$ & $\mathrm{Z}$ \\
\hline 1 & 6 & -0.586375 & 1.507662 & 0.001141 \\
\hline 2 & 6 & 74 & 0.420238 & -0.021968 \\
\hline 3 & 6 & 634154 & 0.831348 & -0.024450 \\
\hline 4 & 6 & -0.123038 & -1.049201 & -0.026817 \\
\hline 5 & 6 & -0.81 & -1.467748 & 89359 \\
\hline 6 & 1 & 32 & -2.547687 & 1.276432 \\
\hline 7 & 1 & -0.187051 & -1.241285 & 2.159924 \\
\hline 8 & 1 & 77 & -0.982276 & 1.434573 \\
\hline 9 & 1 & 6 & -1.632786 & -0.087610 \\
\hline 10 & 1 & 81 & 2.497897 & 9254 \\
\hline 11 & 1 & 05 & 1.906805 & -0.039231 \\
\hline 12 & 6 & 97 & 0.017518 & -0.005510 \\
\hline 13 & 1 & 3.4532 & 0.262422 & -0.901279 \\
\hline 14 & 1 & & 0.346041 & 5153 \\
\hline 15 & 1 & 14 & -1.059309 & 0.046406 \\
\hline 16 & 6 & -2.054990 & 1.535170 & 0.052374 \\
\hline 17 & 1 & -2.365761 & 2.169043 & 0.898285 \\
\hline 18 & 1 & -2.420891 & 2.076983 & -0.836301 \\
\hline 19 & 1 & & 0.565898 & 0.116246 \\
\hline 20 & 6 & & -1.421646 & -1.279105 \\
\hline 21 & 1 & -0.419313 & -1.149074 & -2.200517 \\
\hline 22 & 1 & -1.103914 & -2.504059 & -1.294569 \\
\hline 23 & 1 & -1.930846 & -0.948656 & -1.298400 \\
\hline
\end{tabular}

Energy: $\mathrm{HF}=-313.6004304$

Zero-Point Energy Correction: 0.210398

Imaginary Frequency: Minimum (No imaginary frequencies) 


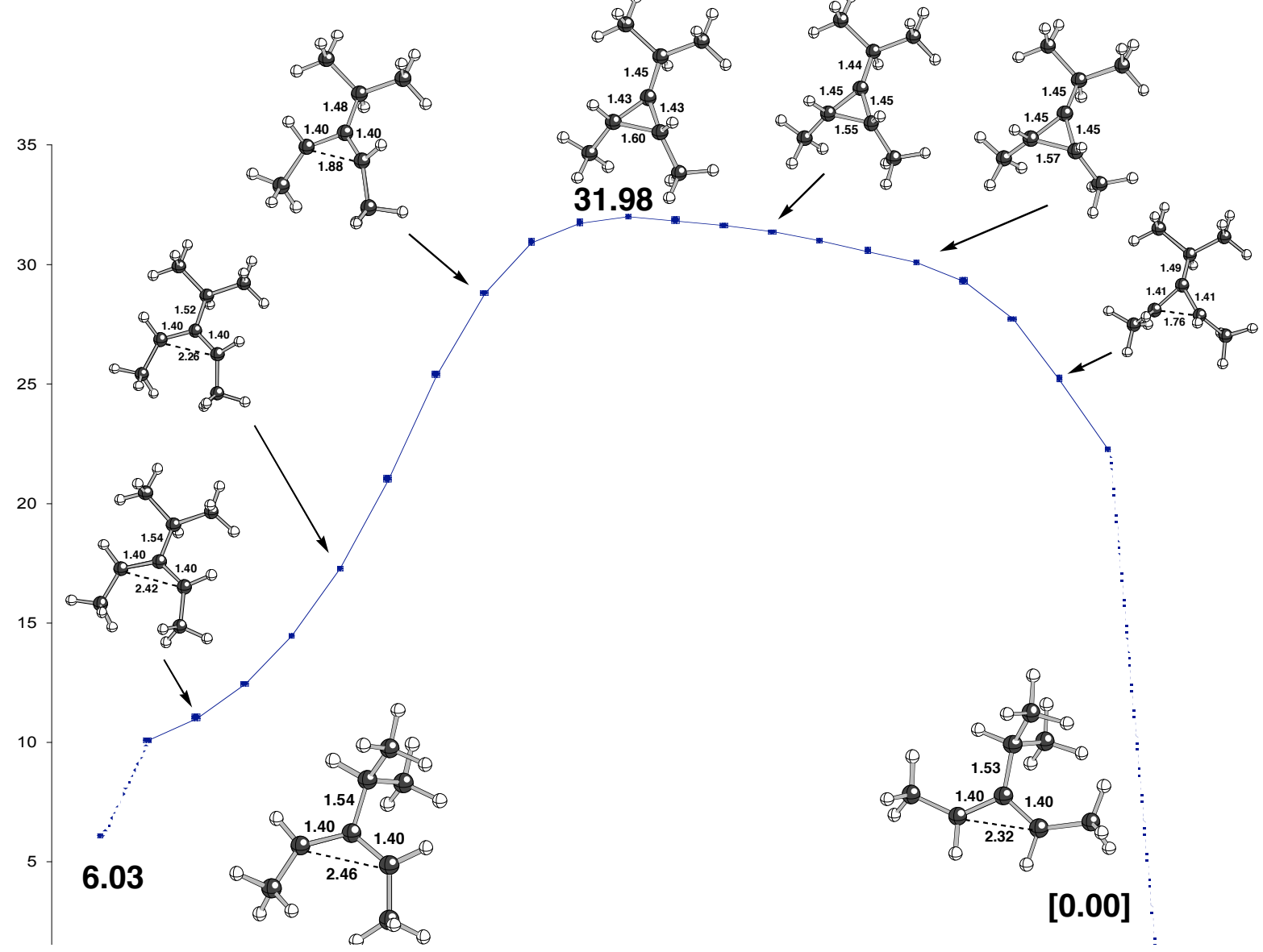




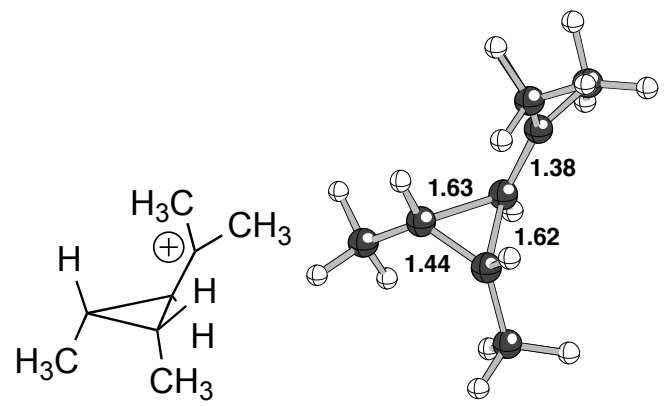

MP2/6-31G(d)

\section{Coordinates:}

Standard orientation:

\begin{tabular}{cccccc} 
Center & \multicolumn{2}{c}{ Atomic } & \multicolumn{2}{c}{ Atomic } & \multicolumn{3}{c}{ Coordinates } & Angstrom \\
Number & Number & Type & X & Y & $Z$ \\
-1 & 6 & 0 & -0.995652 & 0.740474 & 0.457974 \\
2 & 6 & 0 & 0.109790 & -0.042978 & -0.444223 \\
3 & 6 & 0 & -1.013591 & -0.693726 & 0.527442 \\
4 & 6 & 0 & 1.440589 & -0.016168 & -0.087681 \\
5 & 6 & 0 & 2.485778 & -0.087335 & -1.145743 \\
6 & 1 & 0 & 3.240273 & 0.690858 & -0.986963 \\
7 & 1 & 0 & -0.155818 & -0.096167 & -1.495518 \\
8 & 1 & 0 & 3.009825 & -1.048294 & -1.054949 \\
9 & 1 & 0 & 2.075377 & -0.003631 & -2.152493 \\
10 & 6 & 0 & -1.953874 & 1.583065 & -0.339944 \\
11 & 1 & 0 & -2.806155 & 1.827788 & 0.302801 \\
12 & 1 & 0 & -1.480270 & 2.517645 & -0.647396 \\
13 & 1 & 0 & -2.332101 & 1.070418 & -1.224891 \\
14 & 6 & 0 & -1.999497 & -1.582708 & -0.182523 \\
15 & 1 & 0 & -1.555189 & -2.558156 & -0.390631 \\
16 & 1 & 0 & -2.859643 & -1.733020 & 0.478223 \\
17 & 1 & 0 & -2.360452 & -1.152615 & -1.117347 \\
18 & 1 & 0 & -0.512677 & 1.255134 & 1.285996 \\
19 & 1 & 0 & -0.548103 & -1.137652 & 1.404688 \\
20 & 6 & 0 & 1.928502 & 0.092275 & 1.314008 \\
21 & 1 & 0 & 1.160150 & -0.038380 & 2.075564 \\
22 & 1 & 0 & 2.729439 & -0.634990 & 1.483385 \\
23 & 1 & 0 & 2.383078 & 1.083675 & 1.443666 \\
---------------------------------------------------------
\end{tabular}

Energy: MP2 $=-312.4458002$

Zero-Point Energy Correction: 0.214997

Imaginary Frequency: Minimum (No imaginary frequencies) 
MP2/6-31G(d)
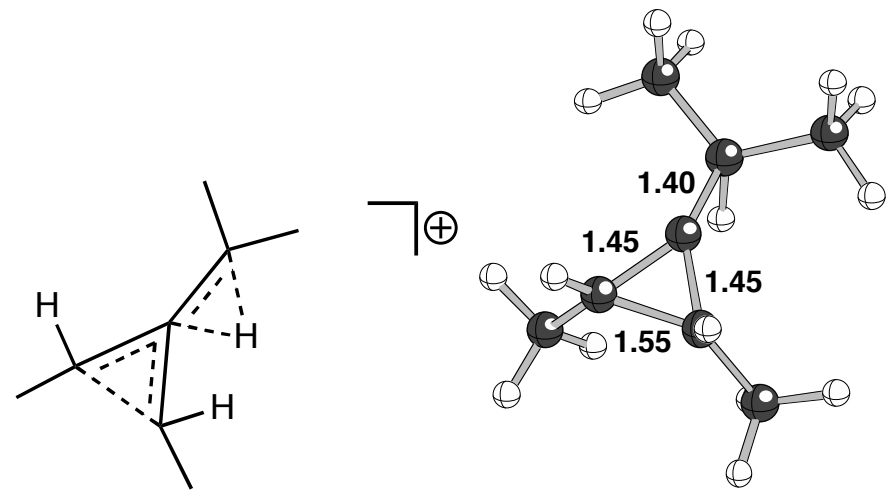

\section{Coordinates:}

Standard orientation:

\begin{tabular}{|c|c|c|c|c|}
\hline \multirow{2}{*}{$\begin{array}{l}\text { Center } \\
\text { Number }\end{array}$} & \multirow{2}{*}{$\begin{array}{l}\text { Atomic } \\
\text { Number }\end{array}$} & \multirow{2}{*}{$\begin{array}{r}\text { Atomic } \\
\text { Type }\end{array}$} & \multicolumn{2}{|c|}{ Coordinates (Angstrom } \\
\hline & & & X $\quad Y$ & $\mathrm{Z}$ \\
\hline 1 & 6 & -1.169865 & 0.776272 & 0.624658 \\
\hline 2 & 6 & 0.045603 & 0.000000 & 0.441931 \\
\hline 3 & 6 & -1.169865 & -0.776272 & 0.624658 \\
\hline 4 & 6 & 1.343380 & 0.000000 & -0.094213 \\
\hline 5 & 6 & 2.136553 & -1.296222 & -0.088014 \\
\hline 6 & 1 & 2.851634 & -1.305769 & -0.912761 \\
\hline 7 & 1 & 0.643826 & 0.000000 & -1.044115 \\
\hline 8 & 1 & 2.697630 & -1.352457 & 0.848557 \\
\hline 9 & 1 & 1.488984 & -2.171690 & -0.162959 \\
\hline 10 & 6 & -1.732454 & 1.651538 & -0.480975 \\
\hline 11 & 1 & -2.804617 & 1.783591 & -0.320403 \\
\hline 12 & 1 & -1.257591 & 2.635344 & -0.471065 \\
\hline 13 & 1 & -1.586401 & 1.204548 & -1.467674 \\
\hline 14 & 6 & -1.732454 & -1.651538 & -0.480975 \\
\hline 15 & 1 & -1.257589 & -2.635343 & -0.471067 \\
\hline 16 & 1 & -2.804617 & -1.783593 & -0.320403 \\
\hline 17 & 1 & -1.586403 & -1.204547 & -1.467674 \\
\hline 18 & 1 & -1.283903 & 1.186029 & 1.631200 \\
\hline 19 & 1 & -1.283903 & -1.186029 & 1.631200 \\
\hline 20 & 6 & 2.136553 & 1.296223 & -0.088014 \\
\hline 21 & 1 & 1.488983 & 2.171690 & -0.162961 \\
\hline 22 & 1 & 2.697629 & 1.352458 & 0.848557 \\
\hline 23 & 1 & 2.851634 & 1.305769 & -0.912761 \\
\hline
\end{tabular}

Energy: MP2 $=-312.3841277$

Zero-Point Energy Correction: 0.211667

Imaginary Frequency: One imaginary frequency $-324.8623 \mathrm{~cm}^{-1}$ 
MP2/6-31G(d)

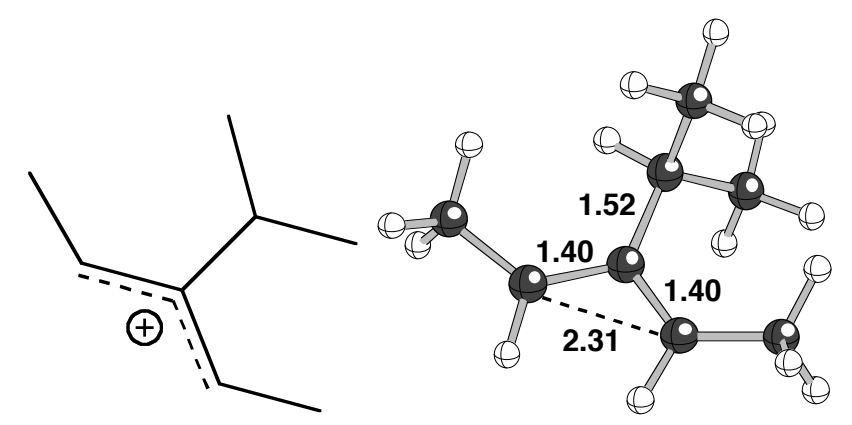

\section{Coordinates:}

Standard orientation:

\begin{tabular}{|c|c|c|c|c|c|}
\hline \multirow{2}{*}{$\begin{array}{l}\text { Center } \\
\text { Number }\end{array}$} & \multirow{2}{*}{\multicolumn{2}{|c|}{$\begin{array}{l}\text { Atomic } \\
\text { Number }\end{array}$}} & \multirow{2}{*}{$\begin{array}{l}\text { Atomic } \\
\text { Type }\end{array}$} & \multicolumn{2}{|c|}{ Coordinates (Angstron } \\
\hline & & & & $\begin{array}{ll}\mathrm{X} & \mathrm{Y}\end{array}$ & $\mathrm{Z}$ \\
\hline 1 & 6 & 0 & 1.106008 & -1.210464 & -0.364923 \\
\hline 2 & 6 & 0 & -0.000027 & -0.367491 & -0.224251 \\
\hline 3 & 6 & 0 & -1.106037 & -1.210526 & -0.364714 \\
\hline 4 & 6 & 0 & -0.000135 & 1.041372 & 0.336077 \\
\hline 5 & 6 & 0 & -1.243597 & 1.816123 & -0.127920 \\
\hline 6 & 1 & 0 & -1.159471 & 2.851904 & 0.208450 \\
\hline 7 & 1 & 0 & -0.000599 & 0.982190 & 1.434219 \\
\hline 8 & 1 & 0 & -1.299908 & 1.825999 & -1.220837 \\
\hline 9 & 1 & 0 & -2.180596 & 1.426176 & 0.267117 \\
\hline 10 & 6 & 0 & 2.394502 & -1.132616 & 0.355911 \\
\hline 11 & 1 & 0 & 2.711164 & -2.138845 & 0.646374 \\
\hline 12 & 1 & 0 & 3.156870 & -0.760329 & -0.345425 \\
\hline 13 & 1 & 0 & 2.368331 & -0.482564 & 1.228561 \\
\hline 14 & 6 & 0 & -2.394391 & -1.132602 & 0.356360 \\
\hline 15 & 1 & 0 & -3.156905 & -0.760530 & -0.344931 \\
\hline 16 & 1 & 0 & -2.710954 & -2.138768 & 0.647143 \\
\hline 17 & 1 & 0 & -2.368089 & -0.482305 & 1.228830 \\
\hline 18 & 1 & 0 & 0.994848 & -2.073494 & -1.023108 \\
\hline 19 & 1 & 0 & -0.994941 & -2.073645 & -1.022791 \\
\hline 20 & 6 & 0 & 1.243653 & 1.816181 & -0.126897 \\
\hline 21 & 1 & 0 & 2.180348 & 1.426416 & 0.269029 \\
\hline 22 & 1 & 0 & 1.300943 & 1.825945 & -1.219766 \\
\hline 23 & 1 & 0 & 1.159099 & 2.851992 & 0.209274 \\
\hline
\end{tabular}

Energy: MP2 $=-312.4220288$

Zero-Point Energy Correction: 0.215254

Imaginary Frequency: Minimum (No imaginary frequencies) 


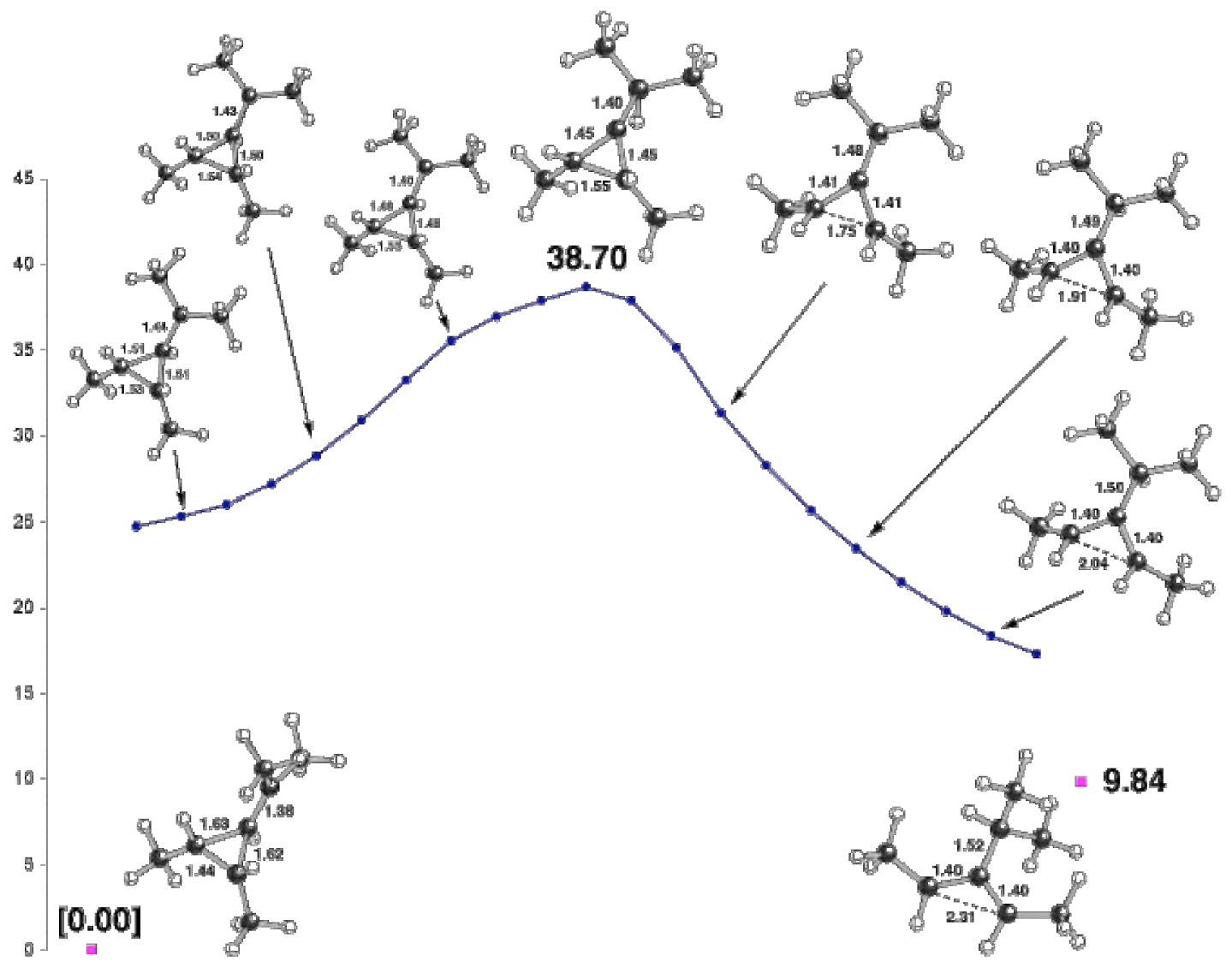




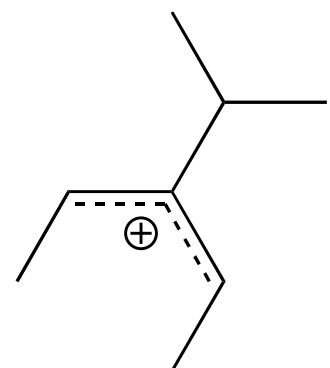

MP2/6-31G(d)

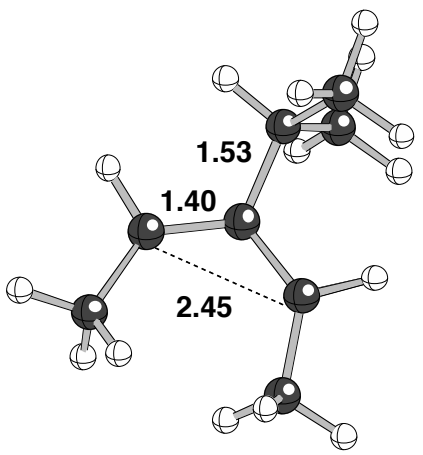

Coordinates:

Standard orientation:

\begin{tabular}{|c|c|c|c|c|}
\hline \multirow{2}{*}{$\begin{array}{l}\text { Center } \\
\text { Number }\end{array}$} & \multirow{2}{*}{$\begin{array}{l}\text { Atomic } \\
\text { Number }\end{array}$} & \multirow{2}{*}{$\begin{array}{l}\text { Atomic } \\
\text { Type }\end{array}$} & \multicolumn{2}{|c|}{ Coordinates (Angstron } \\
\hline & & & $\begin{array}{ll}X & Y\end{array}$ & $\mathrm{Z}$ \\
\hline 1 & 6 & 0.966171 & -1.239589 & -0.100997 \\
\hline 2 & 6 & 0.051896 & -0.186823 & -0.020420 \\
\hline 3 & 6 & 0.449264 & 1.148826 & 0.084343 \\
\hline 4 & 6 & -1.441945 & -0.494340 & -0.040915 \\
\hline 5 & 6 & -2.102035 & -0.086119 & 1.279550 \\
\hline 6 & 1 & -3.151570 & -0.390943 & 1.274254 \\
\hline 7 & 1 & -1.615738 & -0.559422 & 2.137192 \\
\hline 8 & 1 & -2.080244 & 0.999143 & 1.423406 \\
\hline 9 & 1 & -1.541311 & -1.581773 & -0.142912 \\
\hline 10 & 1 & 0.515720 & -2.223700 & -0.244663 \\
\hline 11 & 1 & -0.366555 & 1.860658 & 0.218258 \\
\hline 12 & 6 & 1.794540 & 1.755103 & 0.021039 \\
\hline 13 & 1 & 2.454534 & 1.272137 & -0.701461 \\
\hline 14 & 1 & 1.720341 & 2.820876 & -0.199335 \\
\hline 15 & 1 & 2.273141 & 1.664900 & 1.010965 \\
\hline 16 & 6 & 2.440980 & -1.236868 & 0.004353 \\
\hline 17 & 1 & 2.805494 & -2.241371 & 0.223031 \\
\hline 18 & 1 & 2.871965 & -0.942307 & -0.966844 \\
\hline 19 & 1 & 2.819715 & -0.537481 & 0.751925 \\
\hline 20 & 6 & -2.122968 & 0.156215 & -1.248267 \\
\hline 21 & 1 & -1.648222 & -0.141524 & -2.187511 \\
\hline 22 & 1 & -3.172267 & -0.146605 & -1.288610 \\
\hline 23 & 1 & -2.100418 & 1.248985 & -1.179812 \\
\hline
\end{tabular}

Energy: MP2 $=-312.4199982$

Zero-Point Energy Correction: 0.214448

Imaginary Frequency: Minimum (No imaginary frequencies) 

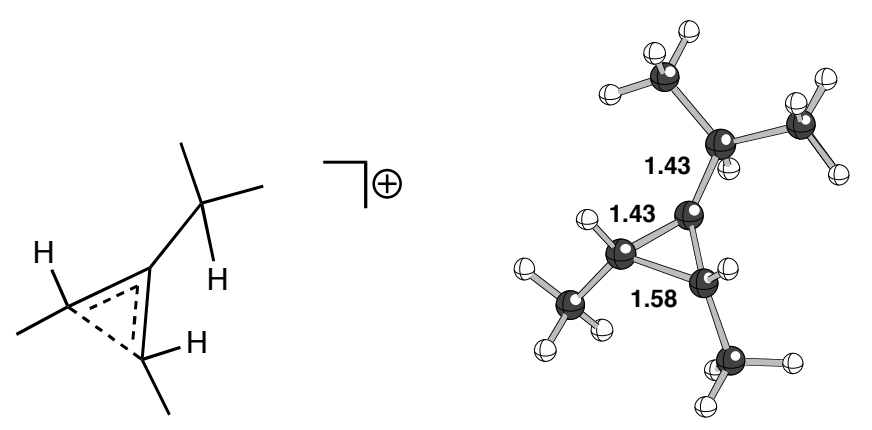

MP2/6-31G(d)

\section{Coordinates:}

Standard orientation:

\begin{tabular}{|c|c|c|c|c|}
\hline \multirow{2}{*}{$\begin{array}{l}\text { Center } \\
\text { Number }\end{array}$} & \multirow{2}{*}{$\begin{array}{l}\text { Atomic } \\
\text { Number }\end{array}$} & \multirow{2}{*}{$\begin{array}{l}\text { Atomic } \\
\text { Type }\end{array}$} & \multicolumn{2}{|c|}{ Coordinates (Angstrom } \\
\hline & & & $\begin{array}{ll}\mathrm{X} & \mathrm{Y}\end{array}$ & $\mathrm{Z}$ \\
\hline 1 & 6 & -0.999134 & -0.791684 & 0.539279 \\
\hline 2 & 6 & 0.084863 & 0.000000 & 0.040186 \\
\hline 3 & 6 & -0.999134 & 0.791684 & 0.539279 \\
\hline 4 & 6 & 1.468884 & 0.000000 & -0.318912 \\
\hline 5 & 6 & 2.224032 & 1.296352 & -0.014948 \\
\hline 6 & 1 & 3.168853 & 1.307579 & -0.561122 \\
\hline 7 & 1 & 1.645540 & 2.178090 & -0.299541 \\
\hline 8 & 1 & 2.448396 & 1.347153 & 1.053642 \\
\hline 9 & 1 & 1.226007 & 0.000000 & -1.426047 \\
\hline 10 & 1 & -0.712697 & -1.344459 & 1.441429 \\
\hline 11 & 1 & -0.712697 & 1.344459 & 1.441429 \\
\hline 12 & 6 & -2.045823 & 1.528815 & -0.294402 \\
\hline 13 & 1 & -2.995518 & 1.533407 & 0.243025 \\
\hline 14 & 1 & -1.712570 & 2.559461 & -0.432621 \\
\hline 15 & 1 & -2.190696 & 1.078802 & -1.275180 \\
\hline 16 & 6 & -2.045823 & -1.528815 & -0.294402 \\
\hline 17 & 1 & -1.712570 & -2.559461 & -0.432621 \\
\hline 18 & 1 & -2.995518 & -1.533407 & 0.243025 \\
\hline 19 & 1 & -2.190696 & -1.078801 & -1.275180 \\
\hline 20 & 6 & 2.224032 & -1.296352 & -0.014948 \\
\hline 21 & 1 & 1.645540 & -2.178091 & -0.299541 \\
\hline 22 & 1 & 3.168853 & -1.307579 & -0.561122 \\
\hline 23 & 1 & 2.448396 & -1.347152 & 1.053643 \\
\hline
\end{tabular}

Energy: MP2 $=-312.3809548$

Zero-Point Energy Correction: 0.212487

Imaginary Frequency: One imaginary frequency $-128.6145 \mathrm{~cm}^{-1}$ 

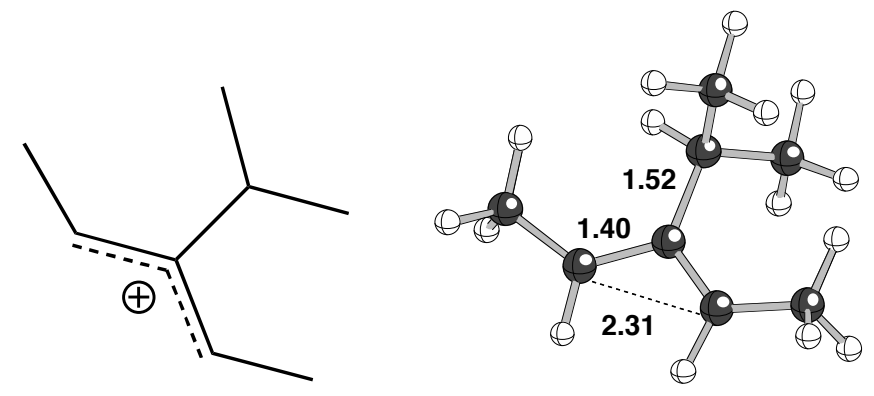

MP2/6-31G(d)

\section{Coordinates:}

Standard orientation:

\begin{tabular}{|c|c|c|c|c|c|}
\hline \multirow{2}{*}{$\begin{array}{l}\text { Center } \\
\text { Number }\end{array}$} & \multirow{2}{*}{$\begin{array}{l}\text { Atomic } \\
\text { Number }\end{array}$} & \multirow{2}{*}{\multicolumn{2}{|c|}{$\begin{array}{l}\text { Atomic } \\
\text { Type }\end{array}$}} & \multicolumn{2}{|c|}{ Coordinates (Angstrom } \\
\hline & & & & X $\quad Y$ & Z \\
\hline 1 & 6 & 0 & 0.630116 & -1.493736 & -0.008675 \\
\hline 2 & 6 & 0 & -0.269227 & -0.430280 & -0.127109 \\
\hline 3 & 6 & 0 & -1.592346 & -0.882069 & -0.126279 \\
\hline 4 & 6 & 0 & 0.094861 & 1.041101 & -0.130704 \\
\hline 5 & 6 & 0 & 0.481865 & 1.539043 & 1.268069 \\
\hline 6 & 1 & 0 & 0.643525 & 2.619590 & 1.236901 \\
\hline 7 & 1 & 0 & -0.307674 & 1.336119 & 1.997561 \\
\hline 8 & 1 & 0 & 1.403947 & 1.076259 & 1.627914 \\
\hline 9 & 1 & 0 & -0.813199 & 1.576332 & -0.425681 \\
\hline 10 & 1 & 0 & 0.208668 & -2.495741 & -0.113792 \\
\hline 11 & 1 & 0 & -1.750181 & -1.958929 & -0.213481 \\
\hline 12 & 6 & 0 & -2.818441 & -0.076436 & -0.016315 \\
\hline 13 & 1 & 0 & -3.303595 & -0.071599 & -1.006334 \\
\hline 14 & 1 & 0 & -3.528415 & -0.570971 & 0.655346 \\
\hline 15 & 1 & 0 & -2.654234 & 0.952075 & 0.299607 \\
\hline 16 & 6 & 0 & 2.077988 & -1.469729 & 0.250367 \\
\hline 17 & 1 & 0 & 2.322578 & -2.223486 & 1.007979 \\
\hline 18 & 1 & 0 & 2.584822 & -1.805730 & -0.668868 \\
\hline 19 & 1 & 0 & 2.471516 & -0.501178 & 0.541486 \\
\hline 20 & 6 & 0 & 1.145363 & 1.376240 & -1.197447 \\
\hline 21 & 1 & 0 & 0.855520 & 0.987724 & -2.178006 \\
\hline 22 & 1 & 0 & 1.229245 & 2.462755 & -1.281308 \\
\hline 23 & 1 & 0 & 2.136410 & 0.991966 & -0.950769 \\
\hline
\end{tabular}

Energy: MP2 $=-312.4301239$

Zero-Point Energy Correction: 0.214790

Imaginary Frequency: Minimum (No imaginary frequencies) 


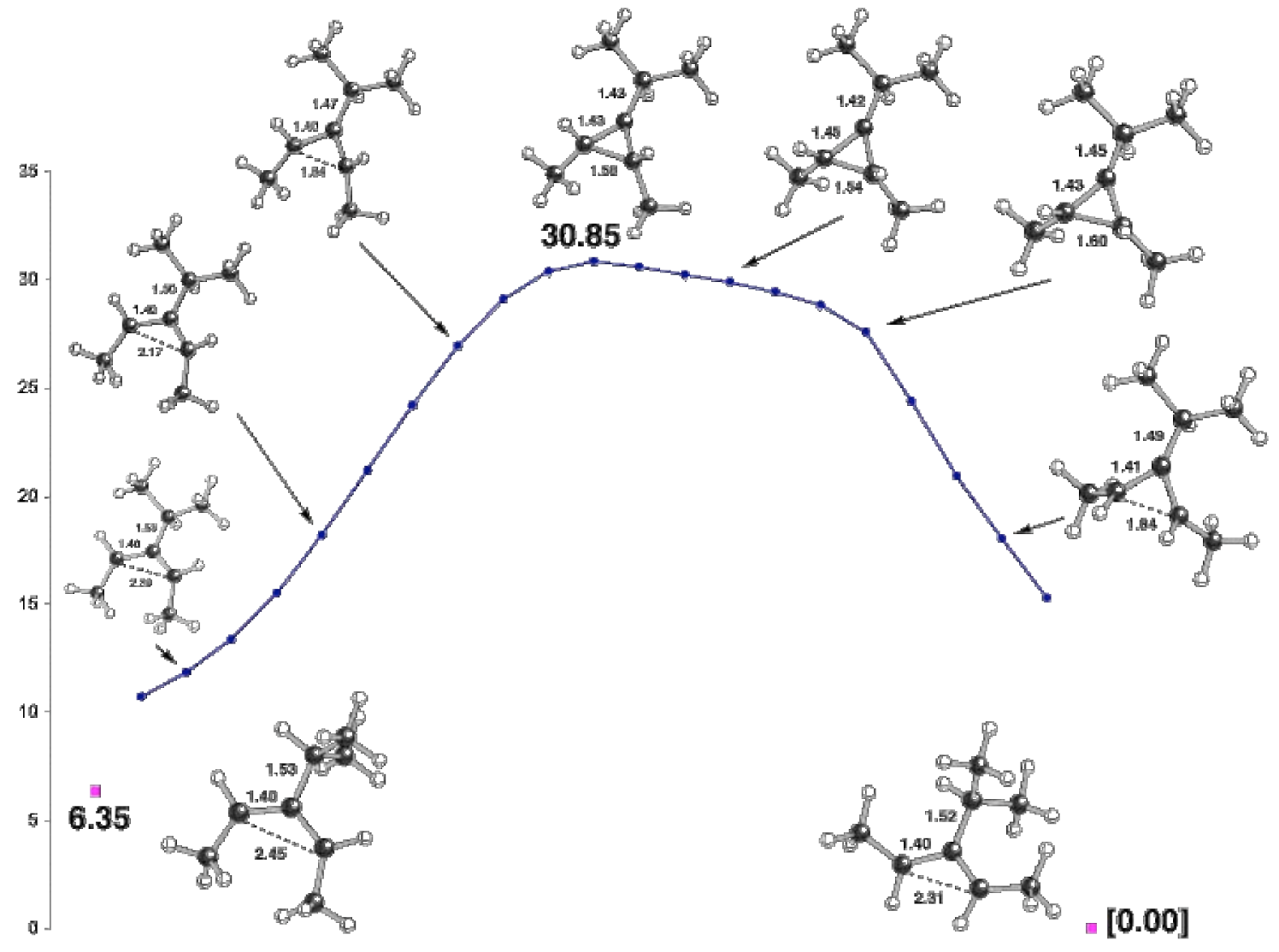


<smiles>CC1(C)[C@H](C)C(C)(C)[C@H]1C</smiles>

b3lyp/6-31G(d)

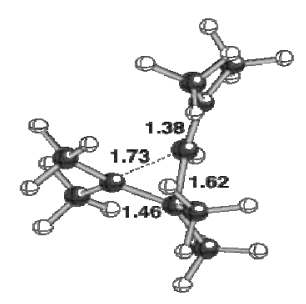

Coordinates:

Standard orientation:

\begin{tabular}{|c|c|c|c|c|c|}
\hline \multirow{2}{*}{$\begin{array}{l}\text { Center } \\
\text { Number }\end{array}$} & \multirow{2}{*}{$\begin{array}{l}\text { Atomic } \\
\text { Number }\end{array}$} & \multirow{2}{*}{\multicolumn{2}{|c|}{$\begin{array}{r}\text { Atomic } \\
\text { Type }\end{array}$}} & \multicolumn{2}{|c|}{ Coordinates (Angstrom } \\
\hline & & & & X $\quad Y$ & $\mathrm{Z}$ \\
\hline 1 & 6 & 0 & 0.986030 & 0.689103 & -0.124225 \\
\hline 2 & 6 & 0 & -0.342218 & -0.057186 & -0.669397 \\
\hline 3 & 6 & 0 & 0.945562 & -0.711661 & 0.278827 \\
\hline 4 & 6 & 0 & -1.661059 & 0.018474 & -0.255395 \\
\hline 5 & 6 & 0 & -2.722361 & -0.355190 & -1.244199 \\
\hline 6 & 1 & 0 & -3.280335 & 0.550981 & -1.523528 \\
\hline 7 & 1 & 0 & -2.329538 & -0.814637 & -2.152873 \\
\hline 8 & 1 & 0 & -3.456517 & -1.026524 & -0.780395 \\
\hline 9 & 6 & 0 & 1.652412 & -1.797291 & -0.510248 \\
\hline 10 & 1 & 0 & 2.650322 & -1.931794 & -0.071576 \\
\hline 11 & 1 & 0 & 1.123651 & -2.750340 & -0.419079 \\
\hline 12 & 1 & 0 & 1.786973 & -1.570250 & -1.567410 \\
\hline 13 & 6 & 0 & 1.850535 & 1.132889 & -1.305906 \\
\hline 14 & 1 & 0 & 1.497738 & 2.098242 & -1.680228 \\
\hline 15 & 1 & 0 & 2.883053 & 1.261539 & -0.961855 \\
\hline 16 & 1 & 0 & 1.859728 & 0.431012 & -2.140850 \\
\hline 17 & 6 & 0 & -2.178970 & 0.424078 & 1.085927 \\
\hline 18 & 1 & 0 & -1.542367 & 1.127055 & 1.619066 \\
\hline 19 & 1 & 0 & -3.182810 & 0.848851 & 0.991873 \\
\hline 20 & 1 & 0 & -2.283712 & -0.477326 & 1.709123 \\
\hline 21 & 1 & 0 & -0.206654 & -0.384568 & -1.694039 \\
\hline 22 & 6 & 0 & 0.854994 & 1.817849 & 0.891367 \\
\hline 23 & 1 & 0 & 1.864867 & 2.177879 & 1.117786 \\
\hline 24 & 1 & 0 & 0.297848 & 2.658241 & 0.467082 \\
\hline 25 & 1 & 0 & 0.402749 & 1.538097 & 1.841638 \\
\hline 26 & 6 & 0 & 0.637053 & -1.145370 & 1.694912 \\
\hline 27 & 1 & 0 & 0.120348 & -2.109761 & 1.705881 \\
\hline 28 & 1 & 0 & 1.597651 & -1.289885 & 2.206316 \\
\hline 29 & 1 & 0 & 0.065137 & -0.430988 & 2.283092 \\
\hline
\end{tabular}

Energy: $\mathrm{HF}=-392.2338694$

Zero-Point Energy Correction: $\mathbf{0 . 2 6 6 8 1 8}$

Imaginary Frequency: Minimum (No imaginary frequencies) 


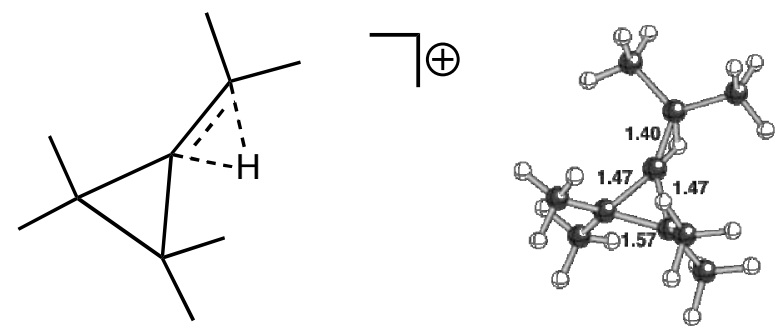

b3lyp/6-31G(d)

Coordinates:

Standard orientation:

\begin{tabular}{|c|c|c|c|c|c|}
\hline \multirow{2}{*}{$\begin{array}{l}\text { Center } \\
\text { Number }\end{array}$} & Atomic & \multicolumn{2}{|c|}{ Atomic } & \multicolumn{2}{|c|}{ Coordinates (Angstror } \\
\hline & Numbe & & Type & X $\quad Y$ & Z \\
\hline 1 & 6 & 0 & 0.924457 & 0.786519 & 0.030535 \\
\hline 2 & 6 & 0 & -0.312247 & -0.000012 & -0.084619 \\
\hline 3 & 6 & 0 & 0.924497 & -0.786510 & 0.030490 \\
\hline 4 & 6 & 0 & -1.708589 & 0.000000 & -0.163876 \\
\hline 5 & 6 & 0 & -2.486547 & -1.297442 & -0.050284 \\
\hline 6 & 1 & 0 & -3.340879 & -1.303305 & -0.732646 \\
\hline 7 & 1 & 0 & -1.863119 & -2.173827 & -0.239484 \\
\hline 8 & 1 & 0 & -2.880786 & -1.366551 & 0.970436 \\
\hline 9 & 6 & 0 & 1.484056 & -1.535655 & -1.181344 \\
\hline 10 & 1 & 0 & & -1.618072 & -1.085408 \\
\hline 11 & 1 & O & & -2.5 & -1.210787 \\
\hline 12 & 1 & & 1.263310 & -1.054582 & -2.136299 \\
\hline 13 & 6 & 0 & 1.483 & 1.535861 & -1.181205 \\
\hline 14 & 1 & 0 & 1.0710 & 2.549395 & -1.210860 \\
\hline 15 & 1 & 0 & 2.57 & 1.618901 & -1.084959 \\
\hline 16 & 1 & & 1.2637 & 1.054575 & -2.136178 \\
\hline 17 & 6 & & -2.486 & 1.297501 & -0.050231 \\
\hline 18 & 1 & 0 & -1.862980 & 2.173853 & -0.239457 \\
\hline 19 & 1 & 0 & -3.340779 & 1.303400 & -0.732596 \\
\hline 20 & 1 & 0 & -2.880 & 1.366639 & 0.970484 \\
\hline 21 & 1 & 0 & -1.105005 & -0.000113 & -1.259751 \\
\hline 22 & 6 & 0 & 1.118959 & 1.560498 & 1.338062 \\
\hline 23 & 1 & 0 & 2.190367 & 1.634168 & 1.553380 \\
\hline 24 & 1 & & 0.724933 & 2.576634 & 1.237125 \\
\hline 25 & 1 & & 0.633 & 1.093352 & 2.197345 \\
\hline 26 & 6 & 0 & 1.1188 & -1.560747 & 1.337886 \\
\hline 27 & 1 & 0 & 0.723776 & -2.576490 & 1.237058 \\
\hline 28 & 1 & 0 & 2.190280 & -1.635486 & 1.552712 \\
\hline 29 & 1 & 0 & 0.633931 & -1.093158 & 2.197404 \\
\hline
\end{tabular}

Energy: $H F=-392.1842231$

Zero-Point Energy Correction: $\mathbf{0 . 2 6 2 8 3 7}$

Imaginary Frequency: One imaginary frequency $\mathbf{- 4 6 3 . 8 6 2 7} \mathrm{cm}^{-1}$ 
b3lyp/6-31G(d)
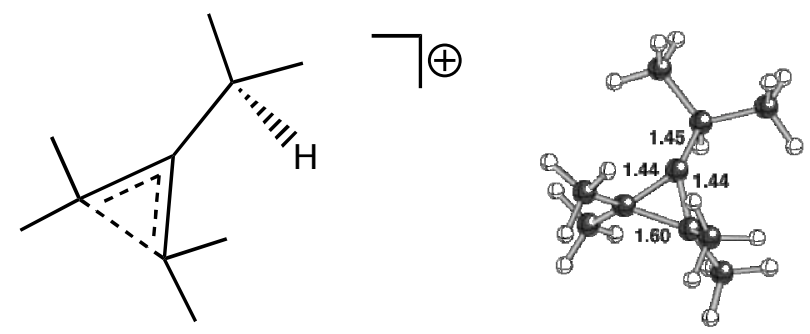

\section{Coordinates:}

Standard orientation:

\begin{tabular}{|c|c|c|c|c|c|}
\hline \multirow{2}{*}{$\begin{array}{l}\text { Center } \\
\text { Number }\end{array}$} & \multirow{2}{*}{$\begin{array}{l}\text { Atomic } \\
\text { Number }\end{array}$} & \multirow{2}{*}{\multicolumn{2}{|c|}{$\begin{array}{r}\text { Atomic } \\
\text { Type }\end{array}$}} & \multicolumn{2}{|c|}{ Coordinates (Angstrom } \\
\hline & & & & $\begin{array}{ll}\mathrm{X} & \mathrm{Y}\end{array}$ & $\mathrm{Z}$ \\
\hline 1 & 6 & 0 & -0.912135 & -0.797915 & 0.030036 \\
\hline 2 & 6 & 0 & 0.285937 & 0.000005 & -0.062824 \\
\hline 3 & 6 & 0 & -0.912133 & 0.797935 & 0.030043 \\
\hline 4 & 6 & 0 & 1.716012 & -0.000005 & 0.193243 \\
\hline 5 & 1 & 0 & 1.660288 & -0.000008 & 1.312527 \\
\hline 6 & 6 & 0 & 2.439735 & -1.296612 & -0.224648 \\
\hline 7 & 1 & 0 & 3.450546 & -1.299907 & 0.190400 \\
\hline 8 & 1 & 0 & 1.918465 & -2.189556 & 0.131148 \\
\hline 9 & 1 & 0 & 2.523133 & -1.349731 & -1.314405 \\
\hline 10 & 6 & 0 & 2.439755 & 1.296600 & -0.224626 \\
\hline 11 & 1 & 0 & 2.523122 & 1.349755 & -1.314384 \\
\hline 12 & 1 & 0 & 1.918516 & 2.189544 & 0.131216 \\
\hline 13 & 1 & 0 & 3.450578 & 1.299856 & 0.190393 \\
\hline 14 & 6 & 0 & -1.080088 & -1.623619 & 1.313848 \\
\hline 15 & 1 & 0 & -2.150919 & -1.780205 & 1.485183 \\
\hline 16 & 1 & 0 & -0.606680 & -2.603977 & 1.203278 \\
\hline 17 & 1 & 0 & -0.662287 & -1.136981 & 2.198453 \\
\hline 18 & 6 & 0 & -1.504096 & -1.513123 & -1.198352 \\
\hline 19 & 1 & 0 & -1.125131 & -2.539232 & -1.218905 \\
\hline 20 & 1 & 0 & -2.593037 & -1.545584 & -1.100246 \\
\hline 21 & 1 & 0 & -1.250734 & -1.041976 & -2.147683 \\
\hline 22 & 6 & 0 & -1.504046 & 1.513117 & -1.198390 \\
\hline 23 & 1 & 0 & -2.593022 & 1.545198 & -1.100565 \\
\hline 24 & 1 & 0 & -1.125415 & 2.539353 & -1.218704 \\
\hline 25 & 1 & 0 & -1.250271 & 1.042199 & -2.147727 \\
\hline 26 & 6 & 0 & -1.080127 & 1.623628 & 1.313852 \\
\hline 27 & 1 & 0 & -0.606739 & 2.603997 & 1.203298 \\
\hline 28 & 1 & 0 & -2.150965 & 1.780188 & 1.485175 \\
\hline 29 & 1 & 0 & -0.662326 & 1.136993 & 2.198459 \\
\hline
\end{tabular}

Energy: -392.1868882

Zero-Point Energy Correction: 0.264744

Imaginary Frequency: One imaginary frequency $-76.6632 \mathrm{~cm}^{-1}$ 

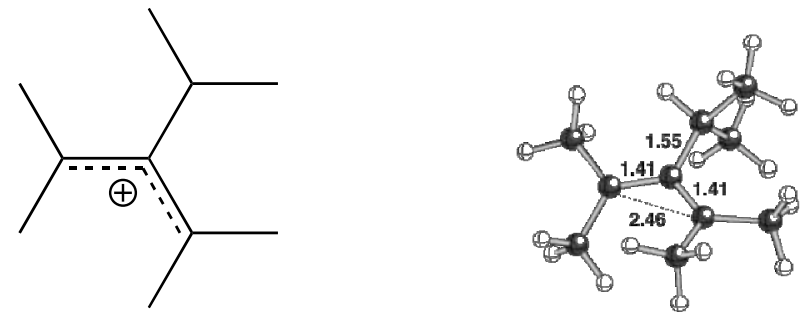

b31yp/6-31G(d)

Coordinates:

Standard orientation:

\begin{tabular}{|c|c|c|c|c|c|}
\hline \multirow{2}{*}{$\begin{array}{l}\text { Center } \\
\text { Number }\end{array}$} & \multirow{2}{*}{$\begin{array}{l}\text { Atomic } \\
\text { Number }\end{array}$} & \multirow{2}{*}{\multicolumn{2}{|c|}{$\begin{array}{r}\text { Atomic } \\
\text { Type }\end{array}$}} & \multicolumn{2}{|c|}{ Coordinates (Angstrom } \\
\hline & & & & $\begin{array}{ll}X & Y\end{array}$ & $\mathrm{Z}$ \\
\hline 1 & 6 & 0 & 0.025647 & 1.354964 & 0.034604 \\
\hline 2 & 6 & 0 & -0.097631 & -0.046257 & -0.073476 \\
\hline 3 & 6 & 0 & -1.364212 & -0.670526 & -0.073412 \\
\hline 4 & 6 & 0 & 1.154339 & -0.960491 & -0.159226 \\
\hline 5 & 6 & 0 & 2.276657 & -0.477925 & -1.099614 \\
\hline 6 & 1 & 0 & 2.937059 & -1.323396 & -1.316844 \\
\hline 7 & 1 & 0 & 1.878325 & -0.120288 & -2.055566 \\
\hline 8 & 1 & 0 & 2.903820 & 0.306068 & -0.670360 \\
\hline 9 & 6 & 0 & -2.634684 & -0.053309 & 0.418585 \\
\hline 10 & 1 & 0 & -3.348966 & 0.045738 & -0.412852 \\
\hline 11 & 1 & 0 & -3.105069 & -0.763146 & 1.112813 \\
\hline 12 & 1 & 0 & -2.532422 & 0.901909 & 0.925859 \\
\hline 13 & 6 & 0 & -1.030038 & 2.337389 & -0.371777 \\
\hline 14 & 1 & 0 & -0.550341 & 3.121667 & -0.972509 \\
\hline 15 & 1 & 0 & -1.852652 & 1.919788 & -0.948000 \\
\hline 16 & 1 & 0 & -1.427985 & 2.858425 & 0.511346 \\
\hline 17 & 6 & 0 & 1.681684 & -1.360467 & 1.236484 \\
\hline 18 & 1 & 0 & 0.895282 & -1.806108 & 1.855597 \\
\hline 19 & 1 & 0 & 2.479788 & -2.101559 & 1.125247 \\
\hline 20 & 1 & 0 & 2.098729 & -0.515234 & 1.792282 \\
\hline 21 & 1 & 0 & 0.795351 & -1.884554 & -0.615035 \\
\hline 22 & 6 & 0 & 1.293755 & 2.022927 & 0.452005 \\
\hline 23 & 1 & 0 & 1.836186 & 2.373716 & -0.438895 \\
\hline 24 & 1 & 0 & 1.052945 & 2.926922 & 1.024376 \\
\hline 25 & 1 & 0 & 1.961332 & 1.391992 & 1.033067 \\
\hline 26 & 6 & 0 & -1.527362 & -2.116578 & -0.432944 \\
\hline 27 & 1 & 0 & -1.077216 & -2.756387 & 0.340981 \\
\hline 28 & 1 & 0 & -2.583153 & -2.387186 & -0.503248 \\
\hline 29 & 1 & 0 & -1.029940 & -2.366733 & -1.375642 \\
\hline
\end{tabular}

Energy: $\mathrm{HF}=-392.2342204$

Zero-Point Energy Correction: 0.267446

Imaginary Frequency: Minimum (No imaginary frequencies) 
Constructed from two IRCs from two different transition states (One at 31.15, the other at $29.48 \mathrm{kcal} / \mathrm{mol}$ )

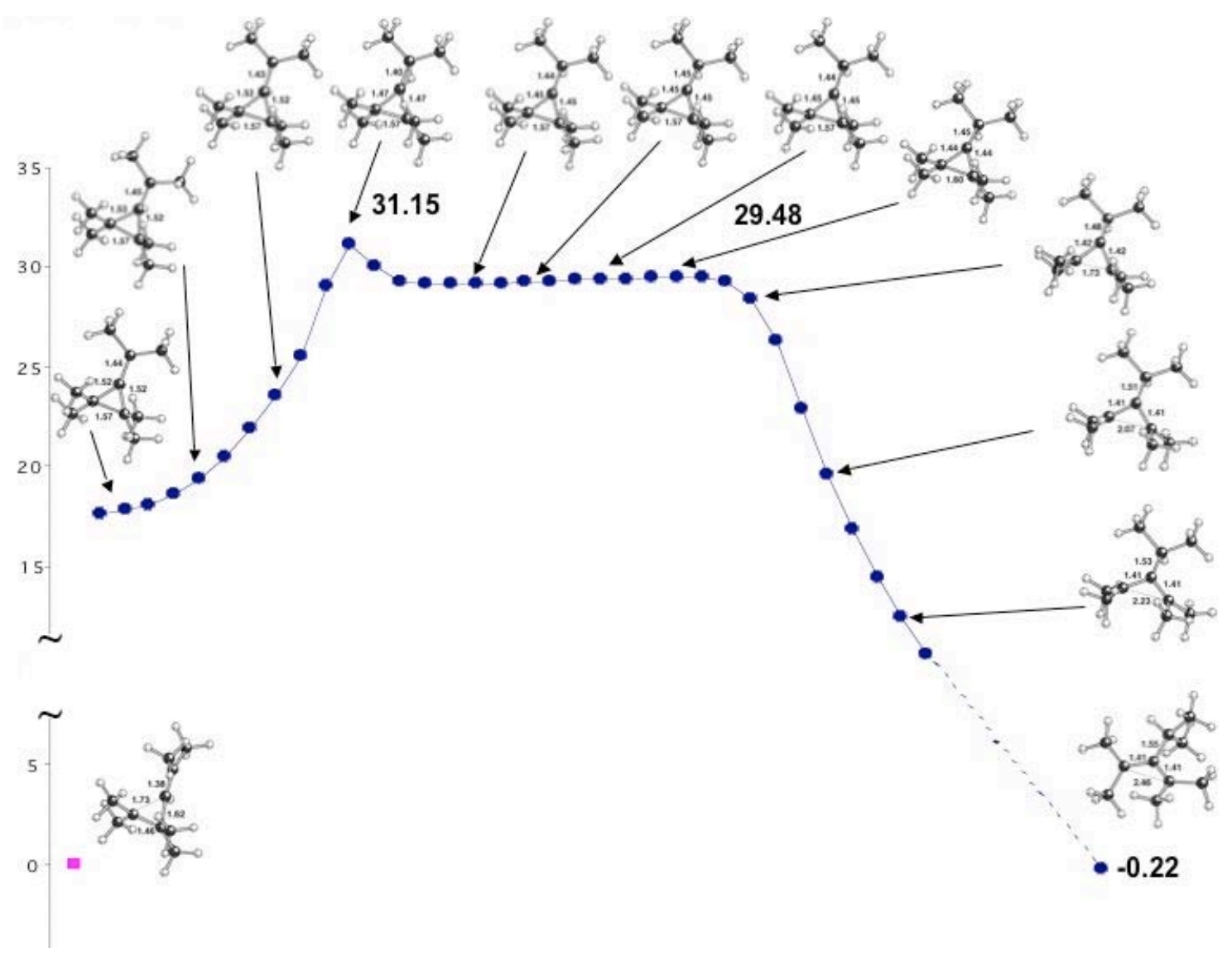




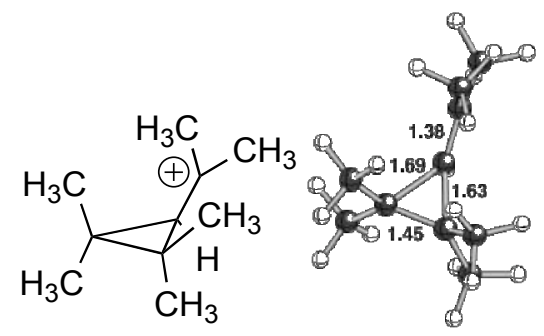

$\mathrm{MP} 2 / 6-31 \mathrm{G}(\mathrm{d})$

\section{Coordinates:}

Standard orientation:

\begin{tabular}{|c|c|c|c|c|c|}
\hline \multirow{2}{*}{$\begin{array}{l}\text { Center } \\
\text { Number }\end{array}$} & \multirow{2}{*}{\multicolumn{2}{|c|}{$\begin{array}{l}\text { Atomic } \\
\text { Number }\end{array}$}} & \multirow{2}{*}{$\begin{array}{l}\text { Atomic } \\
\text { Type }\end{array}$} & \multicolumn{2}{|c|}{ Coordinates (Angstrom } \\
\hline & & & & $X \quad Y$ & $\mathrm{Z}$ \\
\hline 1 & 6 & 0 & -0.994141 & -0.696254 & -0.037795 \\
\hline 2 & 6 & 0 & 0.326663 & 0.016900 & -0.669743 \\
\hline 3 & 6 & 0 & -0.927641 & 0.728914 & 0.199931 \\
\hline 4 & 6 & 0 & 1.638393 & -0.040563 & -0.255139 \\
\hline 5 & 6 & 0 & 2.699474 & 0.174844 & -1.285960 \\
\hline 6 & 1 & 0 & 3.262927 & -0.758579 & -1.413624 \\
\hline 7 & 1 & 0 & 2.303210 & 0.484060 & -2.253737 \\
\hline 8 & 1 & 0 & 3.417911 & 0.921777 & -0.928391 \\
\hline 9 & 6 & 0 & -1.650417 & 1.719644 & -0.686745 \\
\hline 10 & 1 & 0 & -2.643594 & 1.888364 & -0.254129 \\
\hline 11 & 1 & 0 & -1.120951 & 2.675582 & -0.695964 \\
\hline 12 & 1 & 0 & -1.785211 & 1.383550 & -1.713437 \\
\hline 13 & 6 & 0 & -1.839004 & -1.264408 & -1.167088 \\
\hline 14 & 1 & 0 & -1.475219 & -2.261263 & -1.428970 \\
\hline 15 & 1 & 0 & -2.870760 & -1.359962 & -0.812806 \\
\hline 16 & 1 & 0 & -1.845363 & -0.654524 & -2.069642 \\
\hline 17 & 6 & 0 & 2.156869 & -0.267952 & 1.123581 \\
\hline 18 & 1 & 0 & 1.513551 & -0.879658 & 1.749154 \\
\hline 19 & 1 & 0 & 3.147915 & -0.725886 & 1.075070 \\
\hline 20 & 1 & 0 & 2.283598 & 0.709134 & 1.610420 \\
\hline 21 & 1 & 0 & 0.179415 & 0.213613 & -1.726629 \\
\hline 22 & 6 & 0 & -0.835065 & -1.709593 & 1.077070 \\
\hline 23 & 1 & 0 & -1.838178 & -2.074537 & 1.320538 \\
\hline 24 & 1 & 0 & -0.246484 & -2.566592 & 0.739743 \\
\hline 25 & 1 & 0 & -0.409131 & -1.319763 & 1.997913 \\
\hline 26 & 6 & 0 & -0.607497 & 1.315368 & 1.553031 \\
\hline 27 & 1 & 0 & -0.057537 & 2.253675 & 1.442639 \\
\hline 28 & 1 & 0 & -1.564074 & 1.550415 & 2.033457 \\
\hline 29 & 1 & 0 & -0.057835 & 0.659210 & 2.221534 \\
\hline
\end{tabular}

Energy: MP2 $=-390.7819301$

Zero-Point Energy Correction: 0.272638

Imaginary Frequency: Minimum (No imaginary frequencies) 


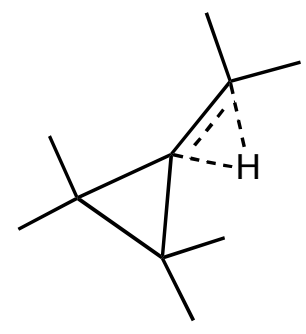

MP2/6-31G(d)

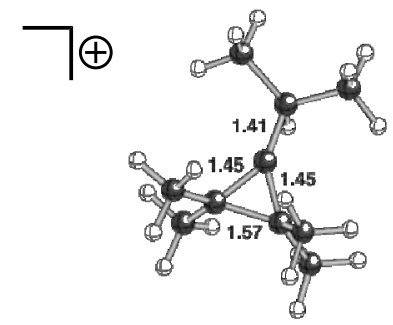

Coordinates:

Standard orientation:

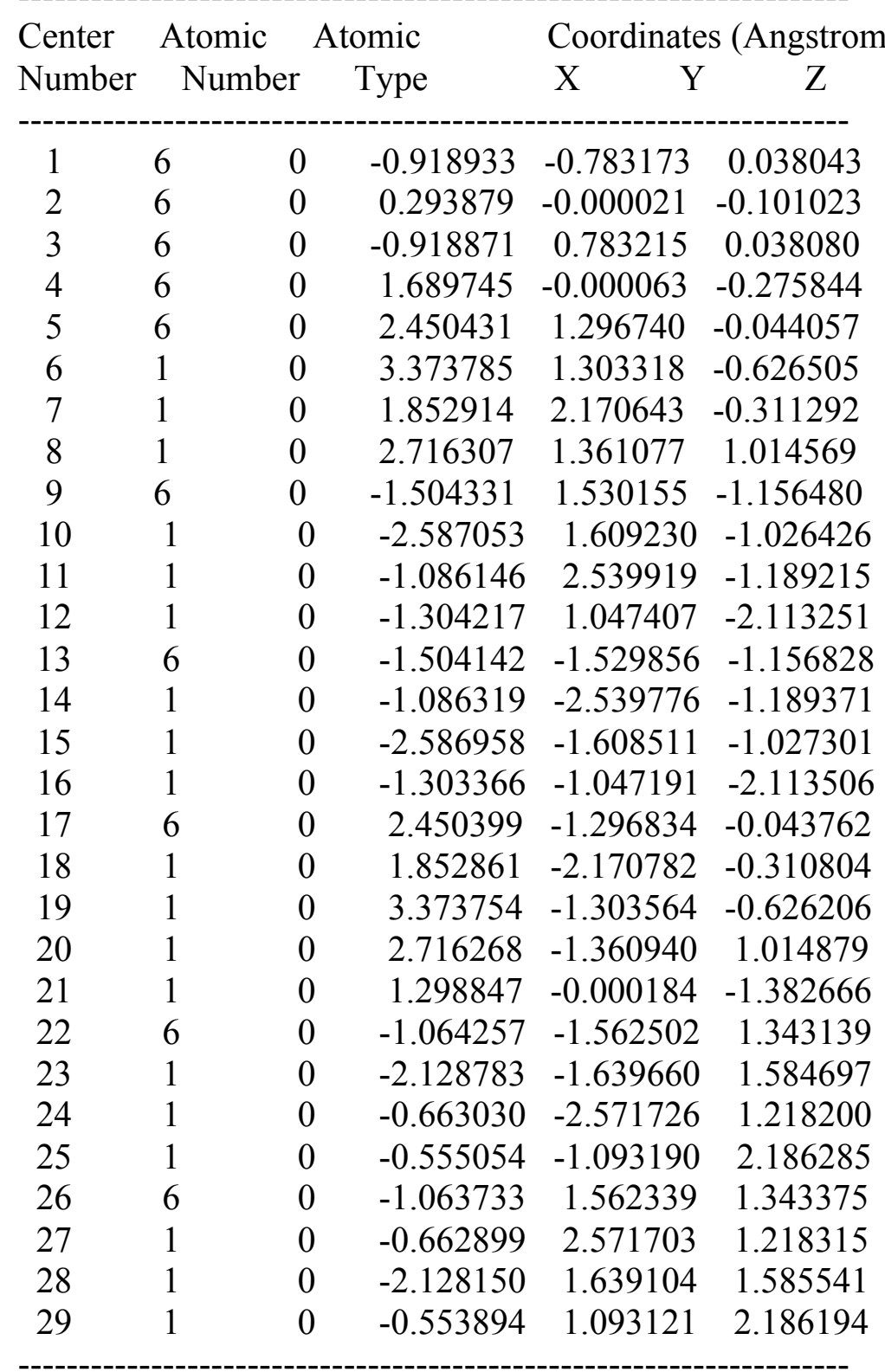

Energy: MP2 $=-390.7302208$

Zero-Point Energy Correction: $\mathbf{0 . 2 6 8 8 6 0}$

Imaginary Frequency: One imaginary frequency $-\mathbf{2 3 4 . 8 9 1 3} \mathrm{cm}^{-1}$ 

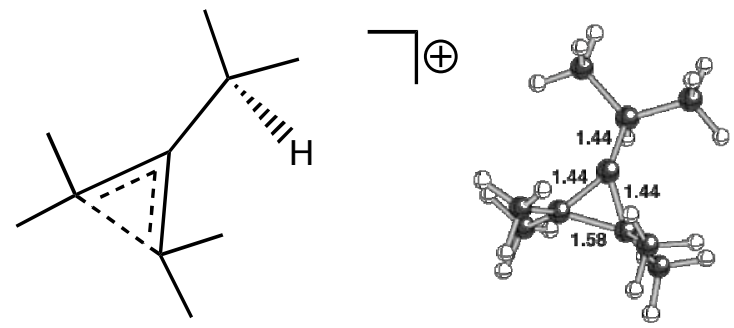

MP2/6-31G(d)

\section{Coordinates:}

Standard orientation:

\begin{tabular}{|c|c|c|c|c|c|}
\hline \multirow{2}{*}{$\begin{array}{l}\text { Center } \\
\text { Number }\end{array}$} & \multirow{2}{*}{\multicolumn{2}{|c|}{$\begin{array}{l}\text { Atomic } \\
\text { Number }\end{array}$}} & \multirow{2}{*}{$\begin{array}{r}\text { Atomic } \\
\text { Type }\end{array}$} & \multicolumn{2}{|c|}{ Coordinates (Angstrom } \\
\hline & & & & X $\quad Y$ & $\mathrm{Z}$ \\
\hline 1 & 6 & 0 & 0.908874 & 0.790170 & 0.027152 \\
\hline 2 & 6 & 0 & -0.288677 & -0.000001 & -0.060877 \\
\hline 3 & 6 & 0 & 0.908876 & -0.790168 & 0.027157 \\
\hline 4 & 6 & 0 & -1.704730 & -0.000003 & 0.190350 \\
\hline 5 & 1 & 0 & -1.603797 & -0.000010 & 1.311546 \\
\hline 6 & 6 & 0 & -2.424903 & 1.292208 & -0.209781 \\
\hline 7 & 1 & 0 & -3.426454 & 1.302030 & 0.224710 \\
\hline 8 & 1 & 0 & -1.886823 & 2.178011 & 0.135042 \\
\hline 9 & 1 & 0 & -2.526024 & 1.339171 & -1.296707 \\
\hline 10 & 6 & 0 & -2.424899 & -1.292212 & -0.209794 \\
\hline 11 & 1 & 0 & -2.526021 & -1.339164 & -1.296721 \\
\hline 12 & 1 & 0 & -1.886816 & -2.178017 & 0.135019 \\
\hline 13 & 1 & 0 & -3.426450 & -1.302042 & 0.224697 \\
\hline 14 & 6 & 0 & 1.091368 & 1.607132 & 1.305384 \\
\hline 15 & 1 & 0 & 2.164064 & 1.752468 & 1.466273 \\
\hline 16 & 1 & 0 & 0.621892 & 2.588251 & 1.195461 \\
\hline 17 & 1 & 0 & 0.675088 & 1.118947 & 2.188810 \\
\hline 18 & 6 & 0 & 1.474578 & 1.507036 & -1.202852 \\
\hline 19 & 1 & 0 & 1.097222 & 2.532751 & -1.207251 \\
\hline 20 & 1 & 0 & 2.564260 & 1.532198 & -1.122641 \\
\hline 21 & 1 & 0 & 1.199321 & 1.040667 & -2.147371 \\
\hline 22 & 6 & 0 & 1.474584 & -1.507042 & -1.202839 \\
\hline 23 & 1 & 0 & 2.564265 & -1.532211 & -1.122620 \\
\hline 24 & 1 & 0 & 1.097221 & -2.532755 & -1.207238 \\
\hline 25 & 1 & 0 & 1.199338 & -1.040674 & -2.147362 \\
\hline 26 & 6 & 0 & 1.091371 & -1.607120 & 1.305396 \\
\hline 27 & 1 & 0 & 0.621898 & -2.588242 & 1.195480 \\
\hline 28 & 1 & 0 & 2.164067 & -1.752451 & 1.466288 \\
\hline 29 & 1 & 0 & 0.675088 & -1.118930 & 2.188818 \\
\hline
\end{tabular}

Energy: MP2 $=-390.7296546$

Zero-Point Energy Correction: 0.269969

Imaginary Frequency: One imaginary frequency $-107.4280 \mathrm{~cm}^{-1}$ 

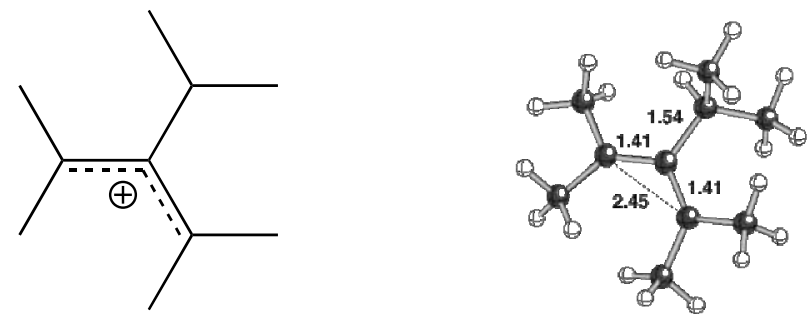

MP2/6-31G(d)

Coordinates:

Standard orientation:

\begin{tabular}{|c|c|c|c|c|c|}
\hline \multirow{2}{*}{$\begin{array}{l}\text { Center } \\
\text { Number }\end{array}$} & \multirow{2}{*}{\multicolumn{2}{|c|}{$\begin{array}{l}\text { Atomic } \\
\text { Number }\end{array}$}} & \multirow{2}{*}{$\begin{array}{r}\text { Atomic } \\
\text { Type }\end{array}$} & \multicolumn{2}{|c|}{ Coordinates (Angstrom } \\
\hline & & & & $\begin{array}{ll}X & Y\end{array}$ & $\mathrm{Z}$ \\
\hline 1 & 6 & 0 & 1.363854 & -0.648714 & -0.082002 \\
\hline 2 & 6 & 0 & 0.089210 & -0.047524 & -0.087733 \\
\hline 3 & 6 & 0 & -0.044802 & 1.347585 & 0.038248 \\
\hline 4 & 6 & 0 & -1.137222 & -0.970191 & -0.178226 \\
\hline 5 & 1 & 0 & -0.774508 & -1.867966 & -0.681562 \\
\hline 6 & 6 & 0 & -1.616132 & -1.417552 & 1.210757 \\
\hline 7 & 1 & 0 & -2.407731 & -2.163622 & 1.098302 \\
\hline 8 & 1 & 0 & -0.805084 & -1.870163 & 1.789169 \\
\hline 9 & 1 & 0 & -2.024007 & -0.590926 & 1.796925 \\
\hline 10 & 6 & 0 & -2.282983 & -0.464344 & -1.062957 \\
\hline 11 & 1 & 0 & -2.914137 & 0.277782 & -0.574592 \\
\hline 12 & 1 & 0 & -1.910309 & -0.047901 & -2.003850 \\
\hline 13 & 1 & 0 & -2.926404 & -1.312730 & -1.311497 \\
\hline 14 & 6 & 0 & 1.545958 & -2.086829 & -0.444877 \\
\hline 15 & 1 & 0 & 2.605829 & -2.341006 & -0.497502 \\
\hline 16 & 1 & 0 & 1.085013 & -2.733691 & 0.314104 \\
\hline 17 & 1 & 0 & 1.072847 & -2.325372 & -1.401651 \\
\hline 18 & 6 & 0 & 2.611215 & -0.011693 & 0.433963 \\
\hline 19 & 1 & 0 & 3.071637 & -0.718335 & 1.135478 \\
\hline 20 & 1 & 0 & 3.335681 & 0.105881 & -0.383599 \\
\hline 21 & 1 & 0 & 2.472969 & 0.935919 & 0.945221 \\
\hline 22 & 6 & 0 & 0.993504 & 2.331893 & -0.391697 \\
\hline 23 & 1 & 0 & 1.811749 & 1.907607 & -0.967668 \\
\hline 24 & 1 & 0 & 0.490738 & 3.087673 & -1.007904 \\
\hline 25 & 1 & 0 & 1.388110 & 2.876044 & 0.476419 \\
\hline 26 & 6 & 0 & -1.306467 & 1.998177 & 0.485168 \\
\hline 27 & 1 & 0 & -1.053083 & 2.892899 & 1.064374 \\
\hline 28 & 1 & 0 & -1.868096 & 2.351121 & -0.390419 \\
\hline 29 & 1 & 0 & -1.948028 & 1.351940 & 1.076384 \\
\hline
\end{tabular}

Energy: MP2 $=-390.7720541$

Zero-Point Energy Correction: 0.273136

Imaginary Frequency: Minimum (No imaginary frequencies) 
Constructed from two IRCs from two different transition states (One at 32.54, the other at 32.80)

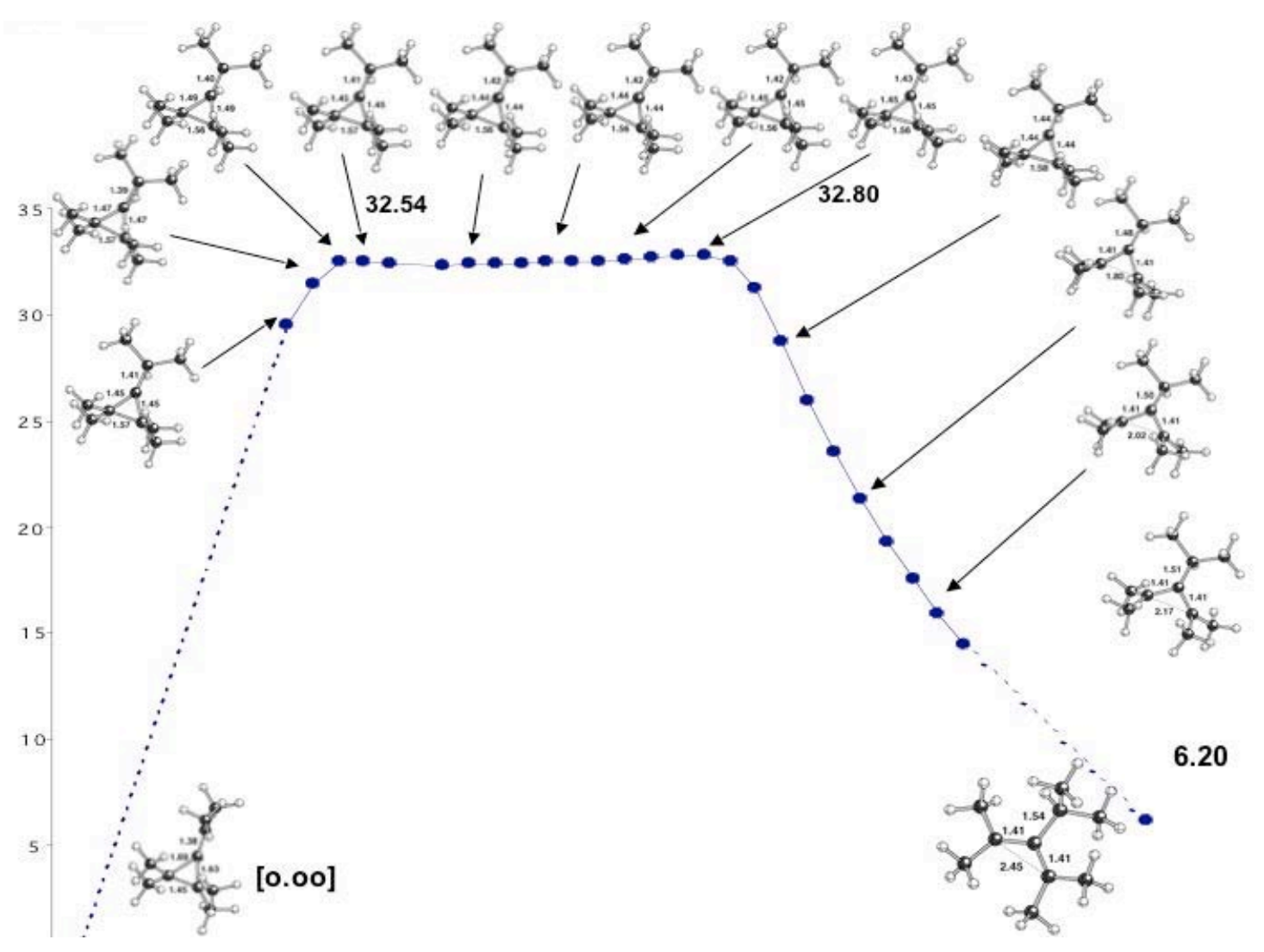

